\title{
WestVirginiaUniversity
}

THE RESEARCH REPOSITORY @ WVU

Graduate Theses, Dissertations, and Problem Reports

2010

\section{Understanding Plasmas through Ion Velocity Distribution Function measurements}

\author{
Saikat Chakraborty Thakur \\ West Virginia University
}

Follow this and additional works at: https://researchrepository.wvu.edu/etd

\section{Recommended Citation}

Chakraborty Thakur, Saikat, "Understanding Plasmas through Ion Velocity Distribution Function measurements" (2010). Graduate Theses, Dissertations, and Problem Reports. 3196.

https://researchrepository.wvu.edu/etd/3196

This Dissertation is protected by copyright and/or related rights. It has been brought to you by the The Research Repository @ WVU with permission from the rights-holder(s). You are free to use this Dissertation in any way that is permitted by the copyright and related rights legislation that applies to your use. For other uses you must obtain permission from the rights-holder(s) directly, unless additional rights are indicated by a Creative Commons license in the record and/ or on the work itself. This Dissertation has been accepted for inclusion in WVU Graduate Theses, Dissertations, and Problem Reports collection by an authorized administrator of The Research Repository @ WVU.

For more information, please contact researchrepository@mail.wvu.edu. 


\title{
Understanding Plasmas through Ion Velocity Distribution Function measurements
}

\author{
Saikat Chakraborty Thakur \\ Dissertation submitted to the College of Arts and Sciences \\ at West Virginia University \\ in partial fulfillment of the requirements \\ for the degree of
}

Doctor of Philosophy

in

Plasma Physics

Earl Scime, Ph. D., Chair

Paul Cassak, Ph. D.

Boyd Edwards, Ph. D.

Fred L. King, Ph. D.

Arthur H. Weldon, Ph. D.

Department of Physics

Morgantown, West Virginia

2010

Keywords: helicon plasma source, velocity distribution function,

electrostatic double layer, ion beam, ion-acoustic wave,

laser induced fluorescence, retarding field energy analyzer, continuous wave cavity ring down spectroscopy 
Abstract

\title{
Understanding Plasmas through Ion Velocity Distribution Function measurements
}

\author{
Saikat Chakraborty Thakur
}

This work concerns measurements of ion velocity distribution functions (IVDF) using three different diagnostic techniques in expanding argon plasmas generated by two different helicon plasma sources. A theoretical prediction by Lieberman et al., of enhanced upstream ionization by electrons accelerating upstream, due to the formation of a stable electrostatic current-free double layer (DL) in an expanding plasma, was verified through laser induced fluorescence (LIF) and retarding field energy analyzer (RFEA) measurements of the IVDF and Langmuir probe measurements of the plasma density. Keeping all other external source parameters constant and varying only the operating frequency of the helicon source HELIX, it was possible to initiate a transition from an unstable to a stable DL. As predicted, the upstream plasma density increased significantly when the stable DL appeared. The instabilities that prevented the formation of a stable DL at low helicon source operating frequency were studied with electrostatic double probes while simultaneously measuring the IVDF. Because the two methods typically used to measure IVDFs in low temperature plasmas require a large population of the target excited state (as in LIF) or are invasive and can be used only for ions in very low density plasmas (as in RFEA), another portion of this work focused on the development of a new method for the non-invasive measurement of IVDFs that is considerably more sensitive than LIF. Continuous wave cavity ring down spectroscopy (CW-CRDS) is a proven, ultrasensitive, cavity enhanced spectroscopic technique that has, in the past, been used to measure the absorption line shapes of particular atomic, ionic, and molecular transitions in test cells. Here we report the first CW-CRDS measurements of the Ar II ion velocity distribution function in a plasma along with conventional LIF measurements of the same ion state in the same plasma. 


\section{Acknowledgements}

I feel this is one of the most difficult parts of writing my thesis. I firmly believe that for what I am today, there are many more people that have helped to shape my life than I can ever remember or even know. Starting from the nurses in a government sponsored small hospital in India in the wee hours of a cold, Friday the $13^{\text {th }}$ night in 1981; the hard working men that used to repair the shaky footbridge that I crossed to go to school in a flood prone area, all the way to those who keep West Virginia University (WVU) functioning in a proper way every day, for whom should I not be thankful? In spite of all the ups and downs of life, I am really lucky to have lots of nice people around me.

From life in general at WVU to being a student of plasma physics, first and foremost I thank my adviser Prof. Earl Scime for giving me the opportunity and the confidence that I needed. From the scientific discussions, to the friendly jokes, you have been my perfect "role model." I earnestly hope that I shall keep your style of advising in mind if someday I have my own students. I sincerely thank all my committee members: Prof. Arthur Weldon, Prof. Fred King, Prof. Boyd Edwards and Prof. Paul Cassak for being very kind to serve on my committee. I also thank Prof. Moreno, Prof. Golubovic, Prof. Barnes and Prof. Koepke for helping me understand a wider spectrum of physics. Many thanks to Prof. Zielinski and Prof. Abdul-Razzaq for setting good examples of being effective instructors for undergraduate students. I also take this opportunity to thank my adviser in India, Prof. Robin Pal of the Saha Institute of Nuclear Physics (SINP), Kolkata for introducing me to the wonderful world of experimental plasma physics. I am also grateful to my Physics teachers who taught me during my Master’s program at the Indian Institute of Technology in Kanpur, India and during my Bachelor's program at St. Xavier's college in Kolkata, India.

I am most grateful to all the staff in our Physics Department. To Tom Milam, Carl Weber, Doug Mathess and Phil Tucker I owe my most sincere gratitude. Without your help I would be nowhere near finishing my thesis work. Thank you Siobhan Byrne, Sandy Johns, Sherry Puskar, and Greg Puskar for making my stay in WVU so pleasant by 
taking care of all the most important yet tedious jobs of proper paperwork so that I can stay legally as a student here and finish my work.

My stay in Morgantown would not have been so nice without all my wonderful friends. Mike Zimmerman, Hong Hong Wei and Jon Blessington were there to discuss homework and qualifier problems. Jason Boyles, Jeff Walker and Luke Shepherd spent a lot of time in the racquetball courts with me. In the labs I am indebted to so many of you: Eric Reynolds, Paul Miller, Josh Miller, Olga Volotskova, Alex Hansen, Ioana Biloiu, Rob Hardin, Saied Houshmandyar, Jerry Carr Jr., Mike Lindon, Stephanie Sears, Dustin McCarren and Matt Galante: I thank all of you for giving me a wonderful environment to work in. I feel myself lucky to be with Jerry and Dusty as my wing commanders in my fight to achieve CRDS. Vivek, Vishal and Sandeep did remind me of the diversity of Indian languages in Hodges hall (even though I don't speak their languages and English was our only common form of speaking!).

Finally I thank my parents and other family members who have been a great source of inspiration that fuelled my enthusiasm over the long journeys whenever I left them and through all these long years when I am so far away from them. I shall befittingly end by thanking my wife, Susmita, who had to bear with my whims and frustrations through most of my graduate student years and for being a part of the endless cell phone conversations that was common to anyone who shares the first floor in Hodges Hall. Her care, love and support gave me the strength to spring back again and again even during the worst nightmarish days in my life.

- Saikat Chakraborty Thakur 


\section{In loving memory of my father:}

\section{Purnendu Chakraborty Thakur}

[1950 - 2006]

who made a complete fool of everyone when he left us on April fool's day in 2006. He is the biggest source of motivation for me to pursue studies in "physics", the subject of my childhood dreams, in an attempt to unlock the secrets of Nature.

(According to my friends who knew him, I have only 5\% of his enthusiasm!) 


\section{Table of Contents}

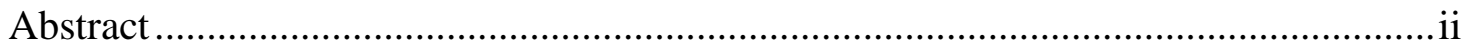

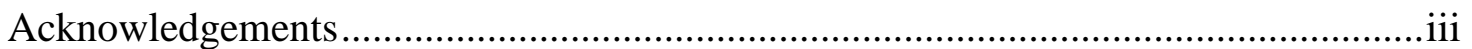

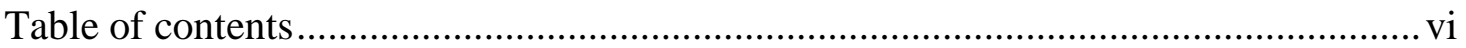

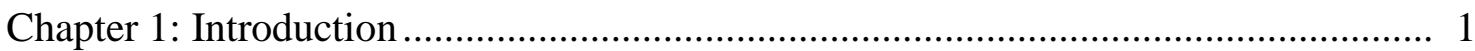

1.1 Velocity distribution function .......................................................................

1.2 Extraction of plasma parameters from velocity distribution functions...................

1.3 Physics insight from ion velocity distribution function measurements .................9

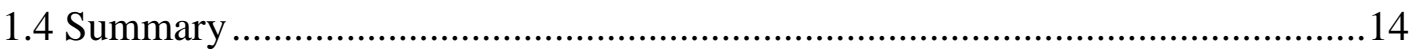

Chapter 1 References .............................................................................................16

Chapter 2: Helicon plasma sources.............................................................................

2.1 Introduction to helicon plasma sources..............................................................17

2.2 Physics of helicon plasma sources ......................................................................18

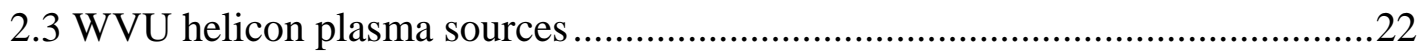

2.3.1 HELIX-LEIA experimental device...........................................................22

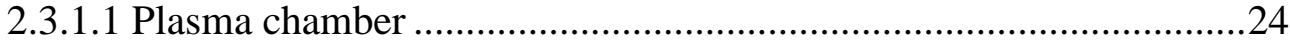

2.3.1.2 Vacuum system ..................................................................................24

2.3.1.3 Magnetic field ...............................................................................26

2.3.1.4 Rf antenna and matching network ...................................................28

2.3.1.5 HELIX-LEIA plasma parameters ........................................................31

2.3.2 CHEWIE experimental device...................................................................32

2.3.2.1 Plasma chamber ................................................................................34

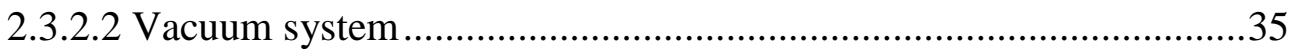

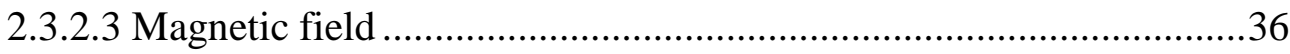

2.3.2.4 Rf antenna and matching network ...................................................37

2.3.2.5 CHEWIE plasma parameters ...............................................................39

Chapter 2 References ..............................................................................................40 
Chapter 3: Standard Plasma Diagnostics ....................................................................43

3.1 Langmuir probe...............................................................................................4

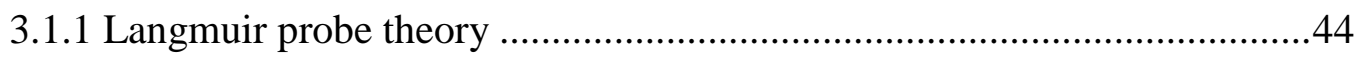

3.1.2 Langmuir probe design ............................................................................49

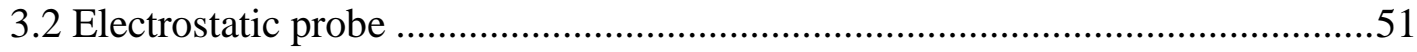

3.2.1 Electrostatic probe theory ………………………....................................51

3.2.2 Electrostatic probe design .........................................................................52

3.2.3 Electrostatic probe analysis.......................................................................53

3.3 Retarding field energy analyzer (RFEA) ………………....................................55

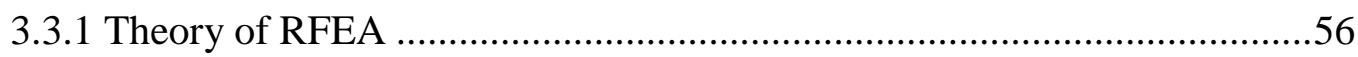

3.3.2 Design and schematics..........................................................................60

3.3.3 Data analysis methodology ........................................................................62

3.4 Laser induced fluorescence (LIF) ..................................................................66

3.4.1 LIF diagnostics apparatus ........................................................................69

3.4.2 LIF with a dye laser ...................................................................................71

3.4.3 LIF with a diode laser ...........................................................................

3.5 Scanning internal probes..................................................................................... 81

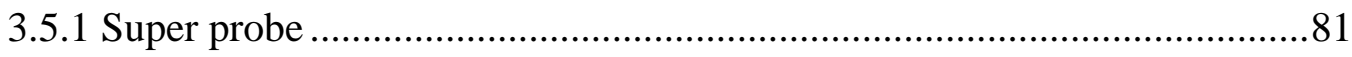

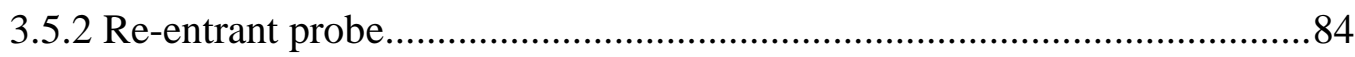

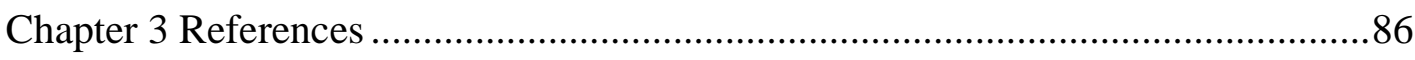

Chapter 4: Comparison of LIF and RFEA methods for measuring IVDFs .....................88

4.1 Shortcomings of conventional RFEA data analysis...............................................8

4.2 An advanced method of RFEA data analysis ........................................................89

4.3 IVDFs obtained from RFEA and LIF measurements ..........................................92

Chapter 4 References ..............................................................................................95

Chapter 5: Studies of electrostatic double layers ........................................................96

5.1 Review of double layers....................................................................................96

5.2 Double layers in helicon plasmas ........................................................................99

5.3 Theory of formation of current-free double layers in expanding

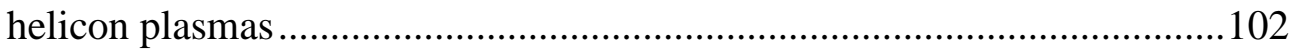




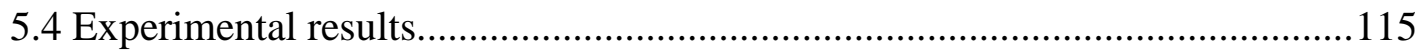

5.4.1 Threshold for ion beam formation ..........................................................117

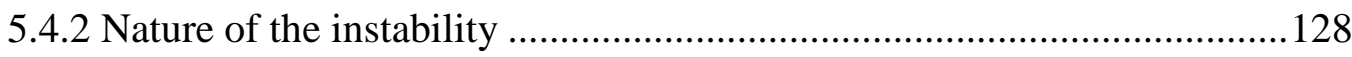

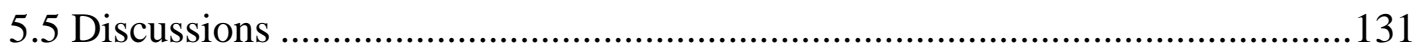

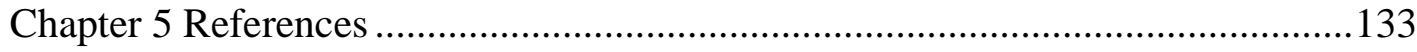

Chapter 6: Cavity ring down spectroscopy as a tool to measure vdf..............................137

6.1 Introduction to Cavity Ring down Spectroscopy...............................................137

6.1.1 Historical background ...............................................................................138

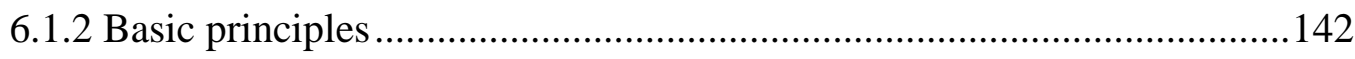

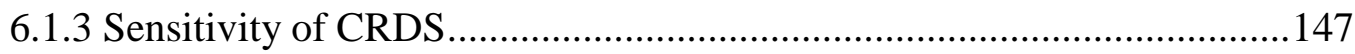

6.1.4 Conventional CRDS experimental apparatus ............................................148

6.2 Continuous wave - cavity ring down spectroscopy (CW-CRDS) .....................149

6.2.1 Properties of optical cavities ....................................................................150

6.2.2 Experimental apparatus for CW-CRDS.....................................................162

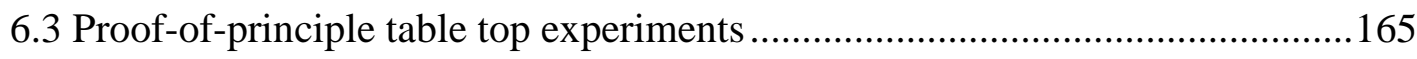

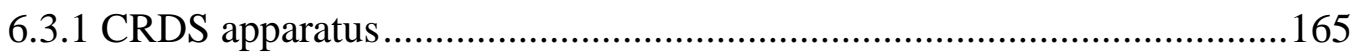

6.3.2 The CW-CRDS tracking circuit..............................................................168

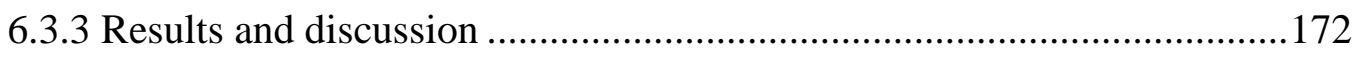

6.3.3.1 Table-top experiment ring down time measurements......................173

6.4 CW-CRDS IVDF measurements in CHEWIE ………......................................175

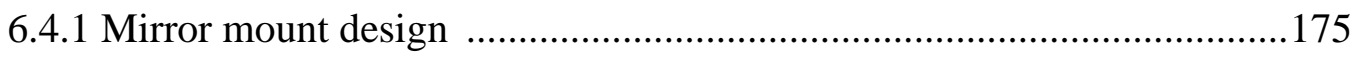

6.4.2 Experimental apparatus for CRDS in CHEWIE ......................................177

6.4.3 Results and discussion .............................................................................178

6.4.3.1 CW-CRDS measurements of Ar-II IVDFs .....................................180

6.4.3.2 Comparison of CW-CRDS and LIF measurements of

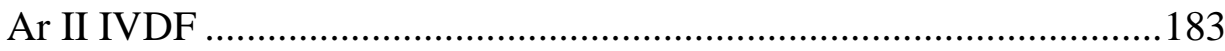

Chapter 6 References .........................................................................................185

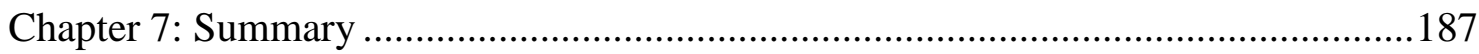

Chapter 7 References ............................................................................................191 


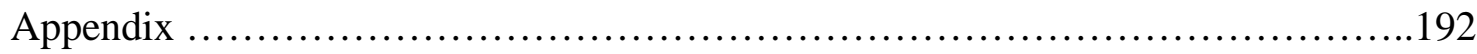

Curriculum Vitae........................................................196 


\section{CHAPTER 1: Introduction}

In science, there are many ways of observing and understanding different physical phenomena. The different methods have their own limitations and thus each method is often only applicable to a specific subset of all possible physical systems. Therefore, it is important to consider the scientific goals of an experiment in terms of the available measurement options. This dissertation, explores a range of physical phenomena in plasmas through measurement of the particle velocity distribution functions (VDF). First, the physical parameters that can be extracted from a VDF measurement will be reviewed. A major component of this work involves the use of measurements of ion velocity distribution functions (IVDF) in expanding helicon plasmas to investigate the physics responsible for the production of high velocity ion beams by spontaneously appearing electrostatic double layers. There are multiple ways of experimentally measuring the IVDF, each with its own limitations. This work concludes with preliminary results that demonstrate a new, non-perturbative, high-sensitivity, method of measuring IVDFs in plasmas.

\subsection{Velocity Distribution Function}

For a system with a large number of particles, it is neither possible nor desirable to determine the exact motion of each and every particle and track them as they evolve in time. Instead, a statistical approach is typically used to track the average motion of a large number of particles. To carry out such a statistical description of plasmas, it is convenient to introduce a six dimensional space, called 'phase space', that has both the conventional three dimensional position coordinates $(x, y, z)$ and the corresponding velocity co-ordinates $\left(v_{x}, v_{y}, v_{z}\right)$. As the physical system evolves in time, at any given moment, the dynamical state of a particle can be represented by a point in this phase space. For a many particle system, the dynamical state of the entire system can be represented by a collection of points in phase space, with one point for each particle. If the number of particles in the system is very large, it is useful to define the average number density of the particles (i.e. points in phase space) in a small volume element of phase space. This density, of points in phase space, is known as the distribution function, $f(\mathbf{r}, \mathbf{v}, t)$, and is defined such that ${ }^{1,2}$ 


$$
d N\left(x, y, z, v_{x}, v_{y}, v_{z}, t\right)=f\left(x, y, z, v_{x}, v_{y}, v_{z}, t\right) d^{3} r d^{3} v
$$

is the number of points in the phase space volume element $d^{3} r d^{3} v=d x . d y \cdot d z \cdot d v_{x} \cdot d v_{y} \cdot d v_{z}$ at any given time $t$. Here, $\mathbf{r}$ and $\mathbf{v}$ denotes the position vector and the velocity vector with spatial components $x, y, z$ and velocity components $v_{x}, v_{y}, v_{z}$, respectively. As time progresses, the long range and short range forces on the particles cause the number of particles in this phase space volume element to. The temporal evolution of $f(\mathbf{r}, \mathbf{v}, t)$ provide a good description of the system. Using the evolution of $f(\mathbf{r}, \mathbf{v}, t)$ to characterize the system eliminates the need to track of the trajectories of individual particles. Instead, the behavior of classes of particles that are closely spaced in phase space (within the volume element $d^{3} r d^{3} v$ ) are tracked. It is generally assumed that the number of particles in the system (corresponding to the number of points in the phase space) is sufficiently large such that the $f(\mathbf{r}, \mathbf{v}, t)$ can be thought to be a continuous function of $\mathbf{r}$ and $\mathbf{v}$. The total number of particles, $N$, in the system, is obtained by integrating the distribution function over the entire phase space

$$
N\left(x, y, z, v_{x}, v_{y}, v_{z}, t\right)=\int_{\substack{\text { phase } \\ \text { space }}} d N\left(x, y, z, v_{x}, v_{y}, v_{z}, t\right)=\int_{-\infty}^{\infty} f\left(x, y, z, v_{x}, v_{y}, v_{z}, t\right) d^{3} r d^{3} v
$$

If the phenomena under investigation are spatially localized, then one can define reduced distribution functions by looking at one point in space, $\mathbf{r}$, within an infinitesimal volume $d^{3} r$, surrounding $\mathbf{r}$. In that case, the general distribution function $f(\mathbf{r}, \mathbf{v}, t)$ reduces to a velocity distribution function $f_{v}(\mathbf{r}, \mathbf{v}, t)$. The velocity distribution function $f_{v}(\mathbf{r}, \mathbf{v}, t)$ is still a function of all seven variables. The number of particles per unit spatial volume (as long as the volume investigated is small enough with respect to the total volume of the system) at position $\mathbf{r}$ and at a given moment in time $t$, that have velocity components between $v_{x}$ and $v_{x}+d v_{x}, v_{y}$ and $v_{y}+d v_{y}$ and $v_{z}$ and $v_{z}+d v_{z}$ is ${ }^{3}$

$$
\operatorname{dn}(x, y, z)=f_{v}\left(x, y, z, v_{x}, v_{y}, v_{z}, t\right) d v_{x} d v_{y} d v_{z}
$$


Integrating the velocity distribution function over the velocity space gives the particle density at $\mathbf{r}$ and $t$. Thus,

$$
n(x, y, z, t)=\int_{V} d n(x, y, z)=\int_{V} f_{v}\left(x, y, z, v_{x}, v_{y}, v_{z}, t\right) d v_{x} d v_{y} d v_{z}
$$

is known as the number density, which is the average number of particles of a particular species, per unit volume at a point, $\mathbf{r}$, in space and time, $t$.

In reality, several species of particles (electrons, ions and neutrals of one or more species) exist simultaneously in plasmas and hence it is customary to define distribution functions for each particle species, $s$, in the plasma as $f_{v s}(\mathbf{r}, \mathbf{v}, t)$.

\subsection{Extraction of Plasma Parameters from Velocity Distribution Functions}

Having defined the distribution function in Section 1.1, the standard rules of statistics can be used to compute various macroscopic parameters that are important for understanding of a plasma system. The average value of a dynamical quantity $g(\mathbf{r}, \mathbf{v})$ in a region $R$ of phase space is given by

$$
\langle g(\mathrm{r}, \mathrm{v})\rangle=\frac{1}{N} \int_{R} g(\mathrm{r}, \mathrm{v}) \cdot f(\mathrm{r}, \mathrm{v}, t) d^{3} r d^{3} v
$$

By making suitable choices for $g(\mathbf{r}, \mathbf{v})$, one can compute various averaged parameters in plasma. When $g(\mathbf{r}, \mathbf{v})$ is equal to 1 , we get the number density of the particles of species s, i.e., Eq. 1.4,

$$
n_{s}=\int_{V} 1 \cdot f_{v s}(\mathrm{r}, \mathrm{v}, t) d^{3} v
$$

Other key parameters are the average velocity, $V s$, of the particles; the kinetic energy density, Ws, which is the average kinetic energy, $(1 / 2) m_{s} v^{2}$, of the particles per unit volume; and the pressure tensor components, $P_{i j}$. The pressure tensor components, 
$P_{i j}$, are defined as the average rate at which momentum is transported in the $i$ direction across surface $j$, in a frame of reference that is moving with average velocity $\boldsymbol{V}$ s. Mathematically, these parameters are defined as

$$
\begin{gathered}
V_{s}=\frac{1}{n_{s}} \int_{V} \mathrm{v} \cdot f_{v s}(\mathrm{r}, \mathrm{v}, t) d^{3} v, \\
W_{s}=\int_{V} \frac{1}{2} m_{s} v^{2} \cdot f_{v s}(\mathrm{r}, \mathrm{v}, t) d^{3} v,
\end{gathered}
$$

and

$$
\vec{P}_{s}=\int_{V} m_{s}\left(\mathrm{v}-\mathrm{V}_{s}\right)\left(\mathrm{v}-\mathrm{V}_{s}\right) \cdot f_{\mathrm{vs}}(\mathrm{r}, \mathrm{v}, t) d^{3} v,
$$

where the integrations are over velocity space, $V$, and $f_{v s}$ is the VDF of species $s$. The quantity $(\mathrm{v}-\mathrm{Vs})(\mathrm{v}-\mathrm{Vs})$ in Eq. 1.9

$$
\left(\mathrm{v}-\mathrm{V}_{s}\right)\left(\mathrm{v}-\mathrm{V}_{s}\right)=\left[\begin{array}{lll}
\left(v_{x}-V_{s x}\right)\left(v_{x}-V_{s x}\right) & \left(v_{x}-V_{s x}\right)\left(v_{y}-V_{s y}\right) & \left(v_{x}-V_{s x}\right)\left(v_{z}-V_{s z}\right) \\
\left(v_{y}-V_{s y}\right)\left(v_{x}-V_{s x}\right) & \left(v_{y}-V_{s y}\right)\left(v_{y}-V_{s y}\right) & \left(v_{y}-V_{s y}\right)\left(v_{z}-V_{s z}\right) \\
\left(v_{z}-V_{s z}\right)\left(v_{x}-V_{s x}\right) & \left(v_{z}-V_{s z}\right)\left(v_{y}-V_{s y}\right) & \left(v_{x}-V_{s x}\right)\left(v_{z}-V_{s z}\right)
\end{array}\right] .(1.1
$$

From these quantities, other important plasma parameters are computed. The average number density, $n_{s}$, and the average velocity, $\mathbf{V}_{s}$, re used to calculate the charge density, $\rho_{q}$, the mass density, $\rho_{m}$, and the current density, $\mathbf{J}_{s}$, corresponding to particles of charge $e_{s}$, and mass, $m_{s}$, through

$$
\rho_{q}=\sum_{s} e_{s} n_{s}
$$




$$
\rho_{m}=\sum_{s} m_{s} n_{s},
$$

and

$$
\mathrm{J}_{s}=\sum_{s} e_{s} n_{s} \mathrm{~V}_{s},
$$

To understand how this theoretical framework enables VDF measurements to provide insight into fundamental plasma physics, a few examples are helpful. Imagine a plasma system where all the electrons are moving at the same velocity, $\mathbf{v}_{\mathrm{e}}=\mathbf{V}_{0}$ and all the ions are at rest $\left(\mathbf{v}_{\mathrm{i}}=0\right)$. The corresponding VDFs are then written in terms of two Dirac delta functions, $\delta$,

$$
f_{e}=n_{0} \delta\left(\mathrm{v}-\mathrm{V}_{0}\right) \text { and } f_{i}=n_{0} \delta(\mathrm{v}) \text {. }
$$

Starting from these velocity distribution functions and Eq. 1.6, it is easy to show that $n_{i}=n_{e}=n_{0}$. The charge density, from Eq. 1.11, is $\rho_{q}=0$ and the average velocity of the electrons, calculated using Eq. 1.7, is $\mathbf{V}_{e}=\mathbf{V}_{0}$ and the average ion velocity is $\mathbf{V}_{i}=0$, thus, confirming the initial assumptions used to define the VDFs. Since the electrons drift with respect to the ions, a net current is present. The current can be calculated from Eq. 1.13 and is given by $\mathbf{J}=-e n_{0} \mathrm{~V}_{0}$. Since there is no spread in velocity with respect to the average electron or ion velocities, the corresponding pressure tensors vanish identically.

As another simple, but more realistic example of a velocity distribution function in plasma, consider a Maxwellian velocity distribution function given by:

$$
f_{s}(\mathrm{v})=n_{s}\left(\frac{m_{s}}{2 \pi k_{B} T_{s}}\right)^{3 / 2} e^{-\left[\frac{m_{s}\left(\mathrm{v}-\mathrm{v}_{s 0}\right)^{2}}{2 k_{B} T_{s}}\right]},
$$

where $k_{B}$ is Boltzmann's constant and $T_{s}$ is the temperature of the particular species. It can be shown from the previous equations that this distribution function has a peak at the 
average velocity $\mathrm{v}=\mathrm{V}_{0}$, and is symmetric about this velocity. The average kinetic energy, defined by Eq. 1.8 is

$$
\left\langle W_{s}\right\rangle=\frac{3}{2} k_{B} T_{s},
$$

and the root-mean-square velocity is

$$
C_{s}=\sqrt{\frac{k_{B} T_{s}}{m_{s}}} .
$$

Figure 1.1 shows a schematic of such a one dimensional velocity distribution function. The width of the distribution is proportional to the square root of the temperature of the species. In many plasma experiments, a measurement of the IVDF with is typically the best, if not the only, method of measuring ion temperature.

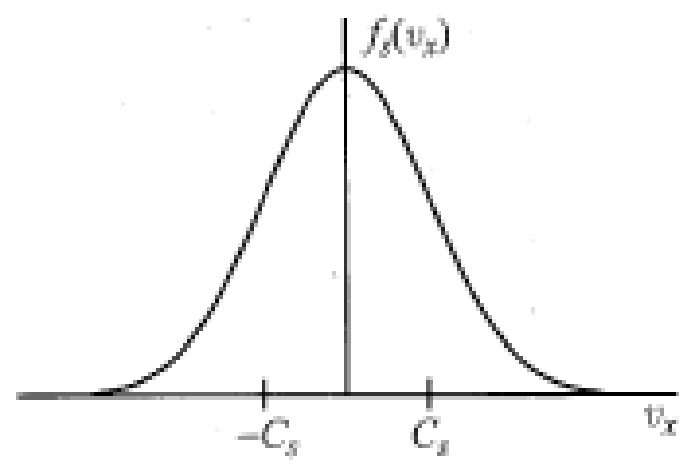

Figure 1.1. Typical Maxwellian velocity distribution function. Figure is from Ref. [1].

The integrals in the pressure tensor are also easy to calculate using Eq. 1.9 and the off diagonal terms corresponding to the matrix in Eq. 1.10 are zero. The diagonal terms are $P_{i j}=n_{s} k_{B} T_{s}$. The pressure tensor is thus diagonal with equal elements, which indicates an isotropic pressure, and reduces to the equation of state of the simple ideal gas law ( $P=$ $\left.n k_{B} T_{s}\right)$. 
Even more realistic distribution functions are anisotropic. One common example of an anisotropic velocity distribution function that occurs in magnetized plasma is the biMaxwellian distribution

$$
f_{s}(\mathrm{v})=n_{s}\left(\frac{m_{s}}{2 \pi k_{B} T_{s \perp}}\right)\left(\frac{m_{s}}{2 \pi k_{B} T_{s \square}}\right)^{1 / 2} e^{-\left[\frac{m_{s}\left(\mathrm{v}_{\perp}\right)^{2}}{2 k_{B} T_{s \perp}}\right]} e^{-\left[\frac{m_{s}\left(\mathrm{v}_{\square}\right)^{2}}{2 k_{B} T_{s \square}}\right]} .
$$

The symbols $\square$ and $\perp$ refer to the direction parallel and perpendicular to the magnetic field. If the co-ordinates are chosen such that the magnetic field is aligned with the z-axis (this is the case in typical cylindrical magnetized plasma machines), then the pressure tensor, defined in Eq. 1.9, is diagonal and has the form

$$
\overrightarrow{\mathrm{P}}=\left[\begin{array}{ccc}
P_{\perp} & 0 & 0 \\
0 & P_{\perp} & 0 \\
0 & 0 & P_{\square}
\end{array}\right],
$$

where $P_{\perp}=n_{s} k_{B} T_{\perp}$ and $P_{\square}=n_{s} k_{B} T_{\square}$. Fig. 1.2 shows a typical contour representation of such a bi-Maxwellian.
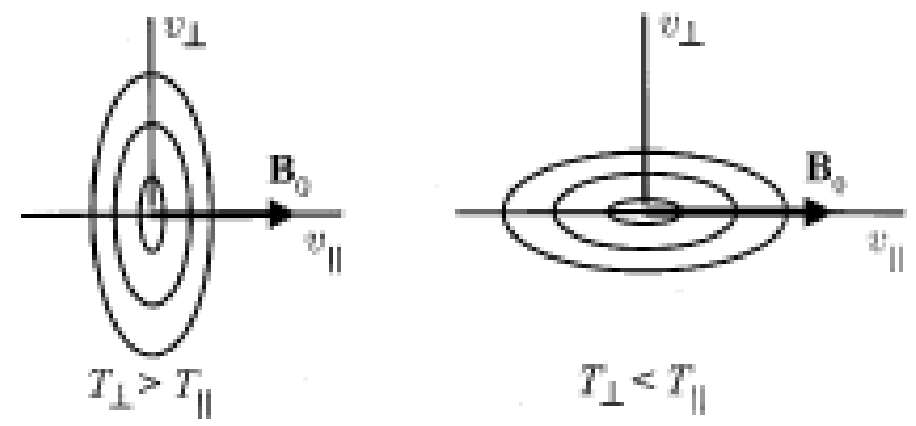

Figure 1.2. Typical bi-Maxwellian velocity distribution function shown for two cases, $T_{\perp}>T_{\square}$ and $T_{\square}>T_{\perp}$, where the perpendicular and parallel directions are with respect to the magnetic field direction. Figure is from Ref. [1]. 
Contour representations of velocity distribution functions are commonly used to aid in the interpretation of two-dimensional VDFs. In a contour representation, lines of constant $f$ are plotted as a function of the two velocity axes. Contours of constant $f\left(v_{x}, v_{y}\right)$ are circles if the VDF is isotropic in $v_{x}$ and $v_{y}$. The VDF given by Eq.1.15 yields concentric circles shifted by the velocity, $\mathbf{V} s_{0}$. A VDF consisting of a drifting Maxwellian along with a beam of particles travelling along the $\mathrm{x}$-direction would have circular contours displaced from the origin and separate region of concentric circles shifted by the beam velocity. Such a VDF is shown in Fig. 1.3 and is representative of the measured argon IVDFs that will be presented in Chapter 5 .

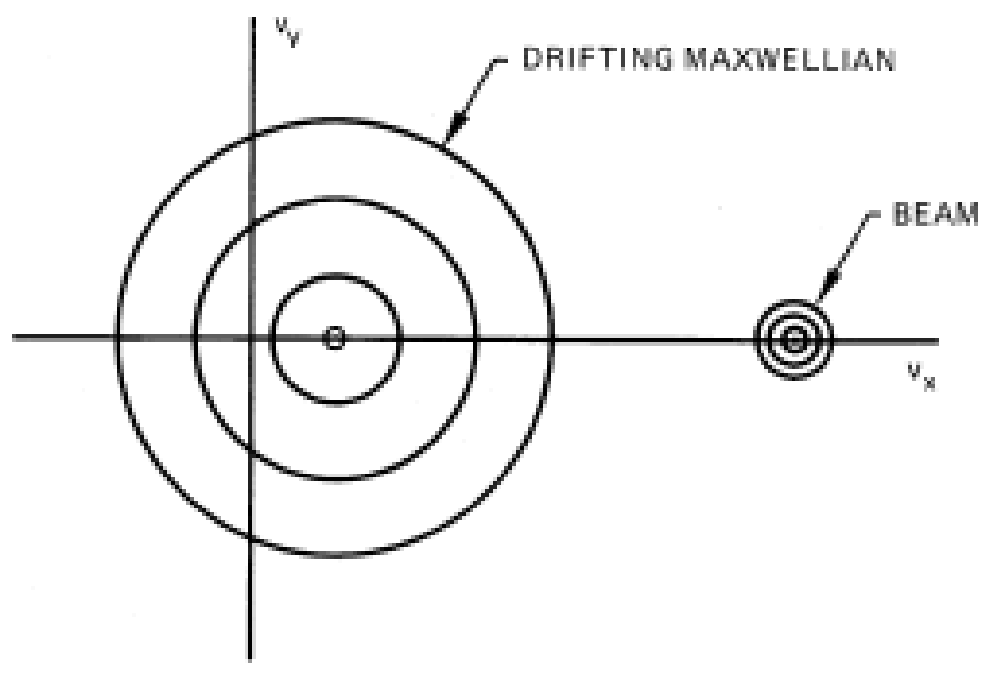

Figure 1.3. Contour representation of constant $f$ for a VDF consisting of a drifting Maxwellian and a "beam" moving in the x-direction. Figure is from Ref. [3].

Therefore, a VDF measurement provides a means of determining the mean flow of a species of particles. If the IVDF is thermalized, the VDF is Gaussian and the full width at half maximum is a measure of the species temperature. Any deviation of the VDF from a Gaussian nature indicates the presence of non-thermal components, which may provide important clues about physical processes occurring in the plasma. Finally, the integral of the VDF is a measure of the species density. 


\subsection{Physics Insight from Ion Velocity Distribution Function Measurements}

Examples of experimental VDF measurements that have provided important insights into the physics of specific plasma systems include measurements in sheaths, wave detection and identification experiments, and experiments with involving evolving electric potential structures.

A sheath is a thin layer of plasma near the boundary of a device (or any finite size structure in plasma, e.g., a divertor or a limiter in fusion plasma devices or a substrate holder in plasma etching and deposition devices) where the potential drops from the plasma potential to zero. Typically a sheath is of the order of a Debye length, ${ }^{4}$ (the length scale beyond which the potential of a charge in plasma is shielded from the rest of the plasma). It was shown by Langmuir ${ }^{5}$ that the ions must arrive at the sheath edge with some velocity derived from weak electric fields extending from the sheath some "considerable distance” into the plasma. The region beyond the sheath is called the "presheath.” Bohm showed that solutions to the equation describing the plasma potential to exist, there is a minimum kinetic energy that the ions must achieve in pre-sheath before they reach the sheath edge. ${ }^{6}$ To study the acceleration of ions due to the pre-sheath, experiments were performed in a laboratory hot-filament multidipole plasma device. ${ }^{7,8,9}$ Fig. 1.4 shows IVDF measurements of argon ions at various locations near the plasmawall boundary. Far away $(z=2 \mathrm{~cm})$ from the boundary at $z=0 \mathrm{~cm}$, the mean ion velocity is small and the shape of the distribution is asymmetric; extending towards the low velocity side. The asymmetry feature arises when charge exchange collisions create low velocity ions from the non-drifting neutral particles of the same species. The ion flow velocity increases throughout the pre-sheath and the ions reach the Bohm speed $\left(C_{1}=\right.$ $2.07 \mathrm{~km} / \mathrm{s}$ ) at a distance of $6 \mathrm{~mm}$ from the boundary. Later work examined the acceleration of ions in multi-species plasmas, a subject of active research. ${ }^{7}$ Such experiments have also been performed at WVU and the measured IVDFs have shown clear acceleration of a single ${ }^{10}$ and multiple ${ }^{11}$ ion species through electrostatic double layers in expanding helicon plasmas. 


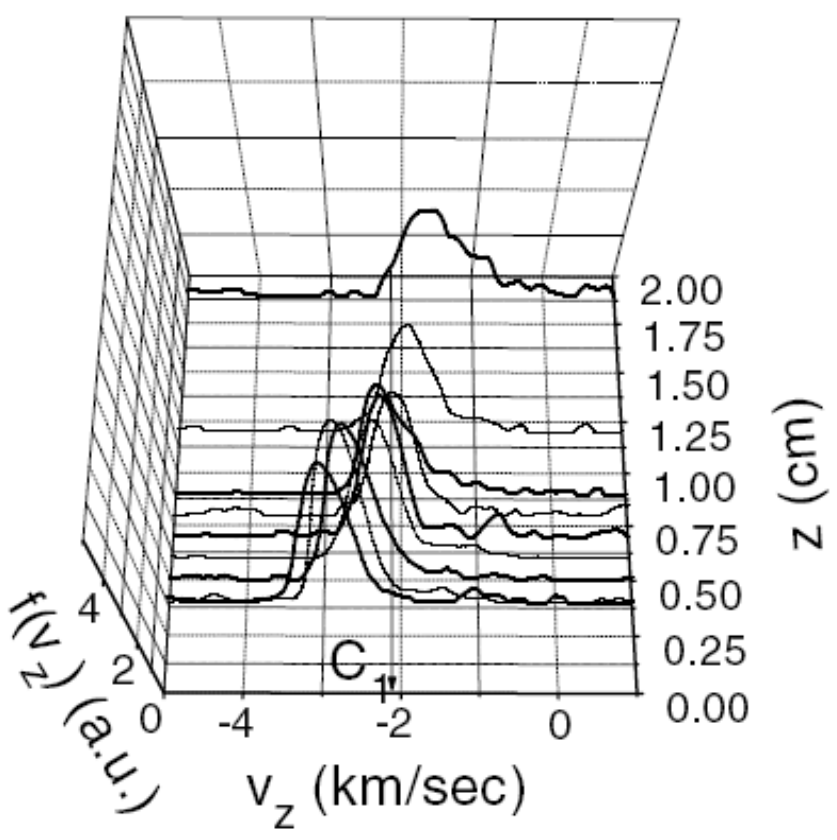

Figure 1.4. A family of IVDFs for argon ions showing that the mean velocity increases up to the Bohm velocity $\left(C_{1}=2.07 \mathrm{~km} / \mathrm{s}\right)$ at the sheath edge as predicted for the formation of a stable sheath. Figure obtained from Ref. [7].

An example of wave detection and study in plasmas through IVDF measurements ${ }^{12}$ comes from one of the earliest experiments using laser induced fluorescence as a tool to measure a IVDF. Electrostatic ion cyclotron waves (EICW) were generated in barium plasma in a single-ended collisionless, fully ionized Q-machine. ${ }^{13}$ The measured IVDFs, as shown in Fig. 1.5, revealed that the ion density decreased within the unstable region of an ion cyclotron wave. Hot ion transport out of the same unstable region also decreased. Figure 1.5a shows the IVDFs near the excitation of the EICW as a function of increasing current density. It is apparent that the ion temperatures $\left(T_{i}\right)$ increase while the total density $(n)$ decreases with increases in the current density $(j)$. The opposite behavior for the density is seen in the IVDFs in Fig. 1.5b, where both $n$ and $T_{i}$ increase with $j$. The IVDF measurements shown in Fig. 1.5b were acquired outside the interaction region of the EICW instability. 


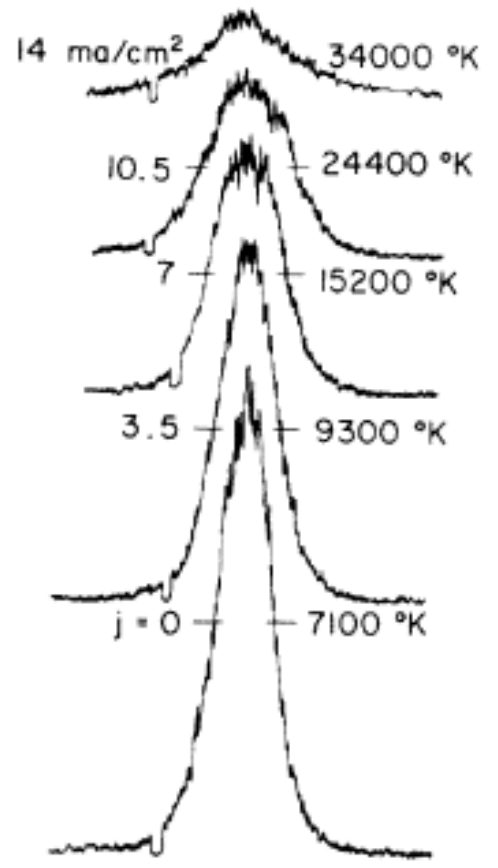

(a)

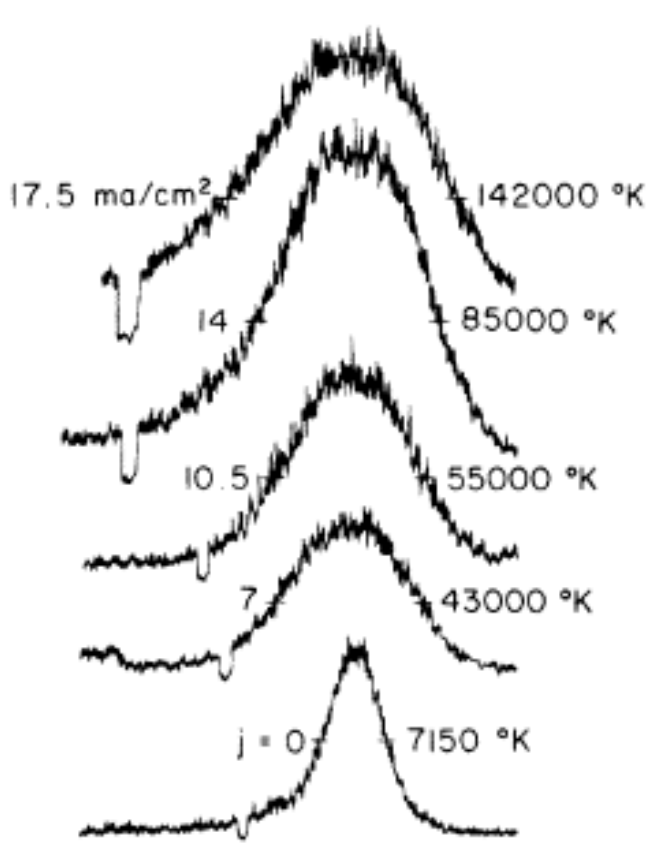

(b)

Figure 1.5. A family of IVDFs for barium ions showing the effects of EICW: (a) At the center of the current filament driving the instabilities and (b) outside the range of the EICW. A blockedlaser-glitch showing the background level is given at the left side of each trace. Figure obtained from Ref. [12].

Another example of a study of wave propagation physics in plasmas was provided by Skiff et. al. ${ }^{14}$ who showed that local measurements of IVDF synchronous with externally launched electrostatic waves carry information concerning the wavelength of the wave perpendicular to the magnetic field. The IVDF measurements also provide a measure of the wave amplitude and phase. All three parameters were directly compared to theory predictions. With linear Vlasov theory ${ }^{15}$ using the method of characteristics ${ }^{16}$, the perturbed VDF is calculated from first principles for a given wave. Figure 1.6 shows an example of a measured perturbed IVDF (and the Fourier transform of the corresponding perturbation) using LIF and a comparison to the theoretical predictions. These observations of plasma dielectric motion confirmed successful launching of EICW and provided the first confirmed of the launching of neutralized ion-Bernstein waves (NIBW) in the plasma. 


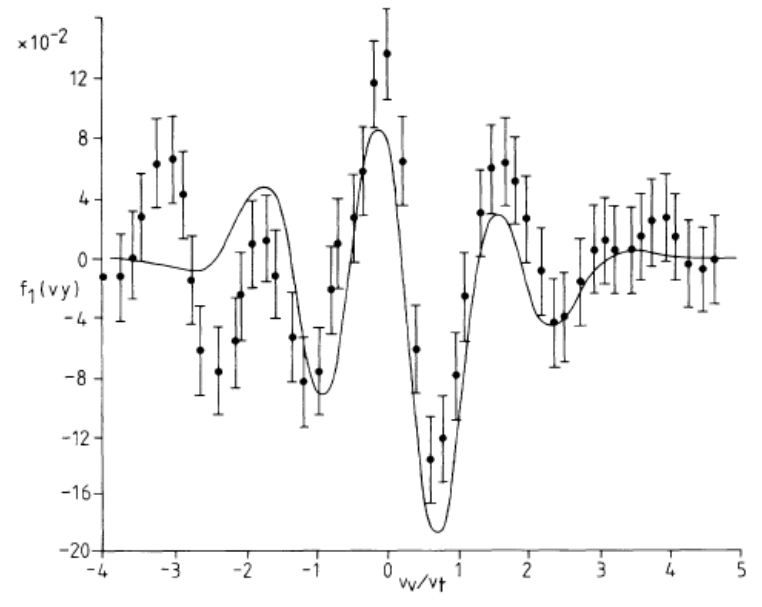

(a)

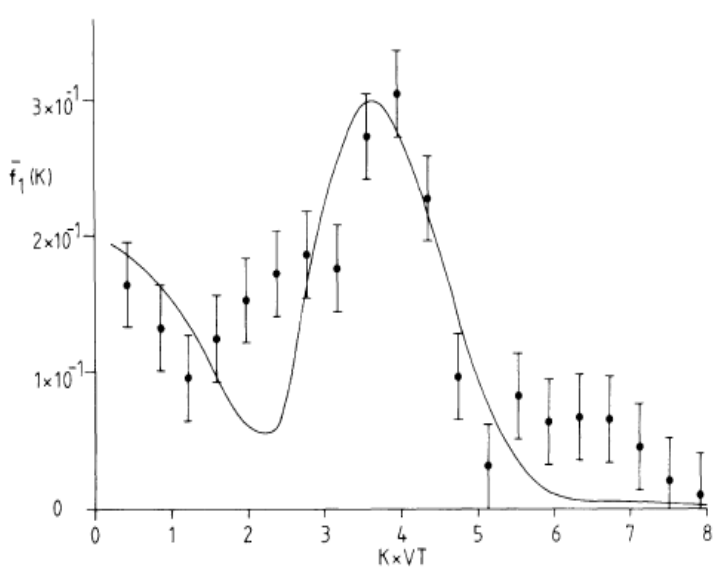

(b)

Figure 1.6. (a) Perturbed component of the IVDF due to NIBW launching. The solid line is theoretically predicted while the dots are the experimental data and (b) the Fourier transform of the corresponding perturbation. The solid line is from theory and the dots are from the data. Figure obtained from Ref. [14].

Nearly identical methods were used in the WVU helicon plasma source to measure the perturbed IVDF for ion cyclotron waves generated in HELIX by an external wave heating antenna. ${ }^{17}$ The IVDF measurements provided non-perturbative measurements of the perpendicular and parallel wave numbers of the waves in the plasma. $^{18}$

A third example of the impact of IVDF measurements on physics understanding of plasma phenomena comes from time resolved measurements of IVDFs. Time resolved LIF measurements in the Versatile Toroidal Facility (VTF) device ${ }^{19}$ yielded the evolution of the IVDF in argon plasma within a magnetic reconnection cycle. ${ }^{20}$ The IVDF measurements showed that magnetic reconnection causes ion heating and that the strongest increase of ion temperatures occurs for the maximum reconnection rate. Figure 1.8 shows the time averaged IVDF with and without the reconnection drive. When reconnection is driven (black squares), the ion temperature increases to $T_{i}=1.1 \mathrm{eV}$ from $T_{i}=0.5 \mathrm{eV}$ for the undriven reconnection case. In addition to the increase in ion 
temperatures, the mean drift velocity increases from $v_{d}=0.29 C_{s}$ to $v_{d}=0.44 C_{s}$ during reconnection, where $C_{s}$ is the ion sound speed.

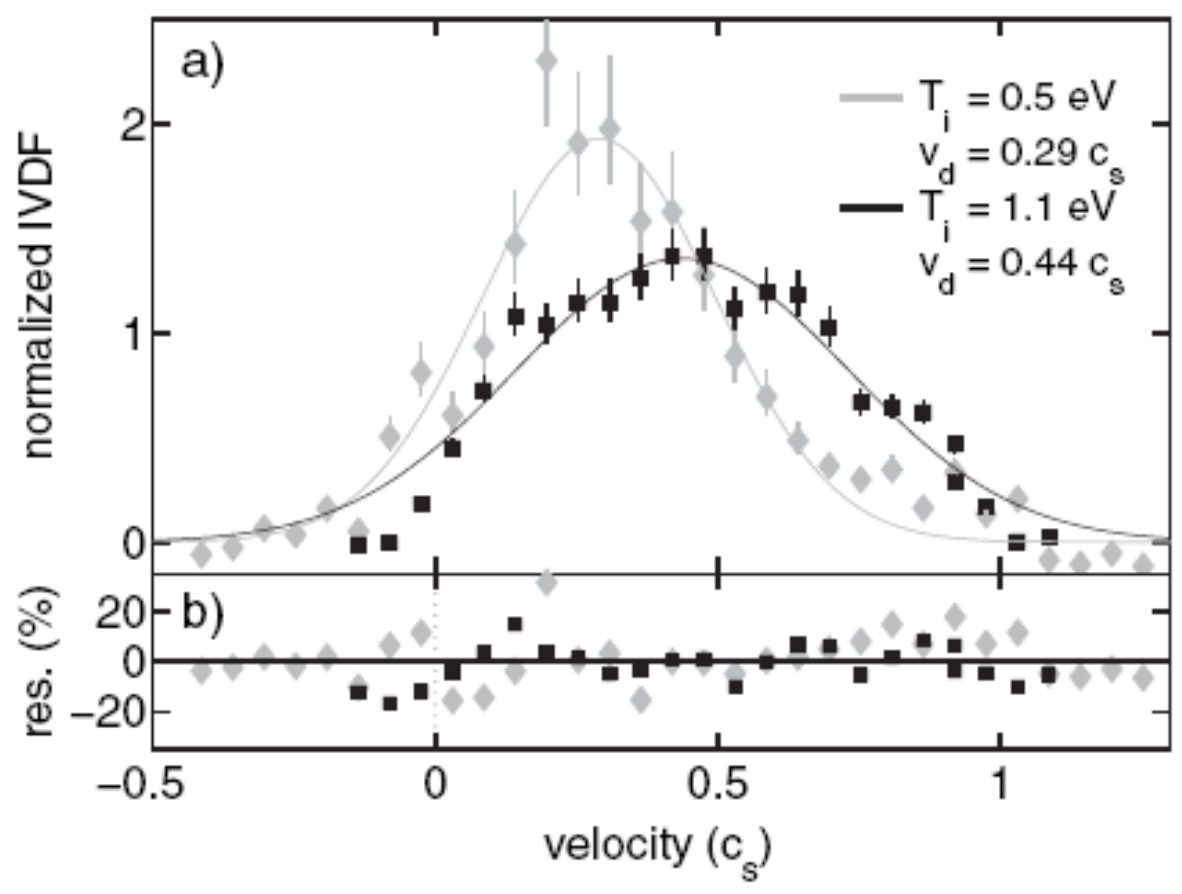

Figure 1.7. (a) LIF measurements of IVDF with (black squares) and without (gray diamonds) drive reconnection. Also shown are the ion temperatures and the mean ion velocities from the Gaussian fist to the two curves. (b) The residua (in \%) of the two least-square fits. Figure obtained from Ref [20].

Figure 1.8 shows the complete temporal evolution of the IVDF in VTF and shows how the ion temperatures vary within the reconnection event. Without reconnection, the ion temperature is almost constant at $0.3 \mathrm{eV}$ throughout the entire shot. But when reconnection is driven, the ion temperature rapidly increases from 1 to $2 \mathrm{eV}$ in 20 milliseconds and then slowly decays to $\sim 1 \mathrm{eV}, 60$ milliseconds later. These changes are correlated with the temporal behavior of the reconnection drive amplitude. Also evident in Fig. 1.9 are strong non-thermal components in the tail region of the IVDF during the reconnection events; ions that are absent in the IVDF without the reconnection drive. Similar time resolved LIF measurements have been used in the WVU helicon plasma 
source group to investigate the physics of double layer formation, a topic that will be revisited in Chapter 5 of this work. ${ }^{21}$

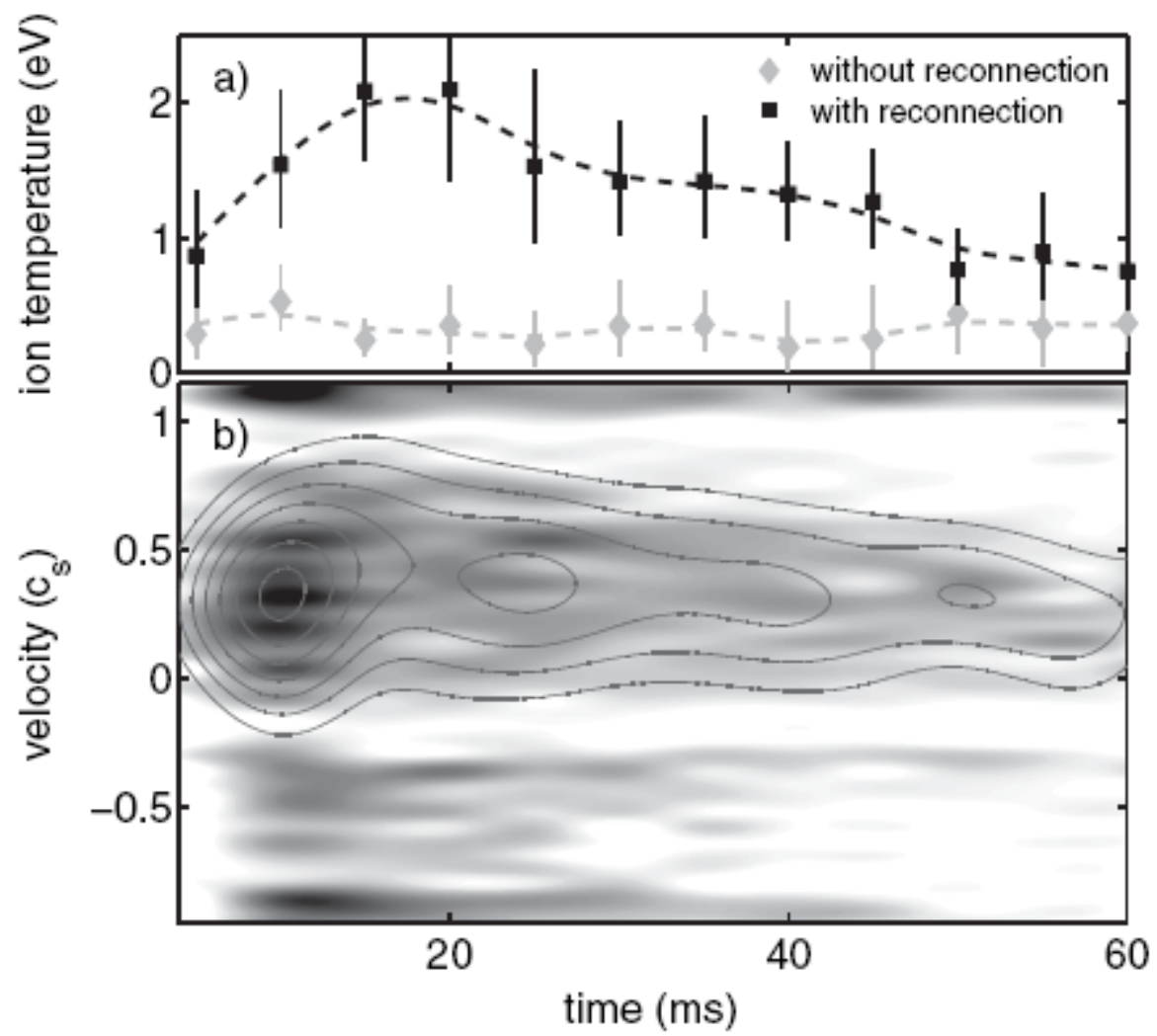

Figure 1.8. (a) Time evolution of the ion temperature in VTF during with (black squares) and without (gray diamonds) driven reconnection and (b) Gray scale plot of the time evolution of the IVDF, which has non thermal components in the tail region. Contours of Gaussians fitted to the thermal components are indicated as solid lines. The decrease in amplitude of the IVDF is due to the decrease in plasma density during the shot in the experiment. Figure obtained from Ref [20].

\subsection{Summary}

As demonstrated by these examples, detailed measurements of IVDFs play an important role in understanding the physics of a wide variety of laboratory plasma experiments. In this work, three methods of measuring IVDFs in plasmas are employed. The retarding field energy analyzer (RFEA) is a standard probe used to measure IVDF in 
various low density plasmas. Even though it is perturbative, it is simple to construct and use. In addition to measuring the IVDF for all ion species simultaneously, a RFEA also provides a measure of the plasma potential. The main problem with RFEA is its relatively large size. In addition to being perturbative, the probe heats up easily and gets destroyed. Thus, RFEAs are used only in low density plasmas or at the edges of higher density, short pulse plasma devices. Laser induced fluorescence is a non-invasive spectroscopic technique for measuring IVDFs. Both RFEA and LIF are standard techniques for measuring IVDFs in plasma. In Chapter 4 of this work, the two methods are compared. In Chapter 5, IVDF measurements by LIF and RFEA are used to investigate the stability of ion beam formation by electrostatic double layers in expanding helicon argon plasma. A theoretical prediction of increased upstream ionization by electrons accelerating upstream towards the plasma source due to stable DL formation is verified in the HELIX helicon source. The instabilities that prevent the formation of a stable DL are examined through electrostatic double probes measurements and discussed in terms of the IVDF measurements in Chapter 5.

LIF is not without its own experimental challenges. For LIF to work, large population of the species in the target initial state (typically a metastable state) is required. Therefore, for many ion species and at low temperatures, the signal-to-noise ratio of any available LIF schemes is not sufficient to permit an IVDF measurement.

To move beyond the limitations of conventional LIF measurements of IVDFs, a portion of this work also focused on the development of a new technique for the noninvasive measurement of IVDFs that is considerably more sensitive than LIF. In Chapter 6, the development and implementation of continuous wave cavity ring down spectroscopy (CW-CRDS) as a tool to measure IVDFs in plasmas is described. CWCRDS is a proven, ultrasensitive, cavity enhanced spectroscopic technique that has, in the past, been used to measure the absorption line shapes of particular atomic, ionic, and molecular transitions in test cells. The results reported here are the first CW-CRDS measurements of the argon IVDF in helicon plasmas. 


\section{Chapter 1 References}

${ }^{1}$ D. A. Gurnett and A. Bhattacharjee, Introduction to Plasma Physics, (Cambridge University Press, 2005)

${ }^{2}$ P. M. Bellan, Fundamentals of Plasma Physics, (Cambridge University Press, 2006)

${ }^{3}$ F. F. Chen, Introduction to Plasma Physics, (Springer, 1974)

${ }^{4}$ P. Debye and P. Huckel, Physikal. Z. 24 (9), 185 (1923)

${ }^{5}$ I. Langmuir, Phys. Rev. 33, 954 (1929)

${ }^{6}$ D. Bohm, The characteristics of Electrical Discharges in Magnetic fields, edited by A. Guthrie and R. K. Wakerling (New York: McGraw-Hill) Chapter 3, p. 77 (1949)

${ }^{7}$ G. D. Severn, X. Wang, E. Ko and N. Hershkowitz, Phys. Rev. Lett. 90, 145001 (2003)

${ }^{8}$ K. N. Leung, N. Hershkowitz and K. R. Mackenzie, Phys. Fluids 19, 1045 (1976)

${ }^{9}$ M. A. Hala and N. Hershkowitz, Rev. Sci. Instrum. 72, 2279 (2001)

${ }^{10}$ X. Sun, A. M. Keese, C. Biloiu, E. E. Scime, A. Meige, C. Charles and R. W. Boswell, Phys. Rev. Lett. 95, 025004, (2005)

${ }^{11}$ I. A. Biloiu, Laser Induced Fluorescence Studies of Ion Acceleration in Single and Multiple Species Expanding Plasmas, Ph. D. Thesis, West Virginia University, Morgantown (2009)

${ }^{12}$ R. A. Stern, D. L. Correll, H. Bohmer and N. Rynn, Phys. Rev. Lett. 37, 833 (1976)

${ }^{13}$ N. Rynn, D. R. Dakin, D. L. Correll and G. Benford, Phys. Rev. Lett. 33, 765 (1974)

${ }^{14}$ F. Skiff and F. Anderegg, Phys. Rev. Lett. 59, 896 (1987)

${ }^{15}$ T. H. Stix, The theory of Plasma Waves, (New York: McGraw-Hill) Pg. 186 (1962)

${ }^{16}$ J. E. Drummond, Phys. Rev. 110, 25 (1958)

${ }^{17}$ J. Kline, E. E. Scime, P. A. Keiter, M. M. Balkey and R. F. Boivin, Plasmas 6, 4767 (1999)

${ }^{18} \mathrm{~J}$. Kline, C. Frank and R. Spangler, First order perturbed velocity distribution theory and measurement , PL-047, West Virginia University, Morgantown (2000)

${ }^{19}$ J. Egedal et. al., Rev. Sci. Instrum. 71, 3351 (2000)

${ }^{20}$ A. Stark, W. Fox, J. Egedal, O. Grulke and T. Klinger, Phys. Rev. Lett. 95, 235005, (2005)

${ }^{21}$ E. E. Scime, I. A. Biloiu, J. Carr Jr., S. Chakraborty Thakur, M. Galante, A. Hansen, S.

Housmandyar, A. M. Keesee, D. McCarren, S. Sears, C. Biloiu and X. Sun, Phys. Plasmas 17, 055701 (2010) 


\section{CHAPTER 2: Helicon Plasma Sources}

\subsection{Introduction to Helicon Plasma Sources}

Helicon waves were first investigated in the early 1960's in solid state plasmas ${ }^{1}$ and in gaseous plasmas. ${ }^{2}$ The basic theory of these helicon waves was studied extensively in that period by Woods, ${ }^{3}$ Klozenberg et al. ${ }^{4}$ and Davies et al. ${ }^{5}$ The generation of high plasma density in a helicon plasma discharge, as we know today, was originally developed by Rod Boswell, ${ }^{6}$ while at Flinders University of South Australia in the early 1970's. Those first experiments produced plasmas with densities of the order of $10^{13} \mathrm{~cm}^{-3}$ with the characteristic argon "blue core". ${ }^{7}$ For approximately twenty years following the first publication, only a modest amount of research was conducted concerning the helicon plasma source

During the 1980's, in a series of experiments, Boswell and co-workers ${ }^{8,9,10}$ investigated the structure and propagation of these helicon waves. Since the early 1990's, there has been a large increase in the number of publications related to the helicon source: largely due to the wide applicability of a plasma source with high density and low temperature. Helicon sources have been constructed for a variety of uses ranging from plasma thrusters, ${ }^{11,12,13,14}$ plasma processing, ${ }^{15,16}$ space relevant experiments ${ }^{17,18}$ and basic

plasma physics experiments. ${ }^{19,20}$ A helicon source is even being considered as a replacement for the current $\mathrm{H}^{-}$ion source for the Spallation Neutron Source [SNS] facility at Oak Ridge National Laboratory [ORNL]. ${ }^{21}$ Excellent reviews are available on the early history of helicon plasma physics research, including the basic theory, by Boswell and Chen. ${ }^{22}$ Helicon research in the following ten years was reviewed by Chen and Boswell. ${ }^{23}$ Most recently, a review by Scime, Keesee and Boswell, following the mini-conference on helicon plasma sources at the $49^{\text {th }}$ Annual American Physical Society-Division of Plasma Physics meeting, discussed topics related to optimal source performance and novel applications of the helicon source. ${ }^{24}$

Helicon discharges are sustained by electromagnetic waves propagating in magnetized plasmas in the so-called "helicon modes". The driving frequency of these discharges is typically in the radio frequency [rf] range of 1 to $50 \mathrm{MHz}$ (for material processing, the industrially licensed radio-frequency of $13.56 \mathrm{MHz}$ is commonly used). 
In contrast to other rf discharges (capacitive and inductively coupled), helicon discharges are considered to be wave heated even though they operate in the rf range. Since the phase velocity of electromagnetic waves in magnetized plasma can be substantially lower than the speed of light, the helicon wavelength is comparable to the discharge system size even at radio frequencies much smaller than the standard frequency of $13.56 \mathrm{MHz}$. The magnetic field in helicon discharges varies from 20 to $2000 \mathrm{G}$ and magnetic field strengths of $\sim 1000 \mathrm{G}$ are often employed for fundamental plasma studies employing helicon sources. Excitation of the helicon waves is provided by an rf antenna that couples to the transverse mode structure of the wave across an insulating chamber wall. The electromagnetic wave mode propagates along the plasma column and the wave energy is transferred to electrons through collisional or collisionless damping mechanisms. Resonant coupling of the helicon mode to the antenna can lead to discontinuous changes in the plasma density for small changes in the source parameters. This phenomenon, known as the "mode jump", is characteristic to a helicon plasma discharge and restricts the operating regime for a given helicon source design. Typically, on-axis peak densities of $\sim 10^{13} \mathrm{~cm}^{-3}$ can be created in a $10 \mathrm{~cm}$ diameter tube with only 1 kilowatt of rf power and 1 kiloGauss of magnetic field strength for a variety of rf antenna configurations.

\subsection{Physics of Helicon Plasma Sources}

Helicon waves are bounded right-hand circularly polarized electromagnetic waves, propagating in the frequency range $\omega_{c i} \ll \omega \ll \omega_{c e}$, where $\omega_{c i}$ is the ion cyclotron frequency, $\omega_{c e}$ is the electron cyclotron frequency and $\omega$ is the wave frequency. Free (unbounded) right-hand circularly polarized electromagnetic waves are typically referred to as "whistler" waves because of their characteristic descending tones as heard during the latter half of World War I. ${ }^{7}$ The waves were inadvertently picked up by radio communication spies while listening for enemy communications and were later determined to be an atmospheric phenomenon; initiated by lightning strikes generating the waves which then propagated along the earth's magnetic field lines.

Helicon waves differ from classical whistler waves in two main aspects: 1) they propagate at frequencies close to the low frequency limit of the electron cyclotron wave, so that electron inertia effects are small, and 2) they are modes of bounded systems; 
hence their purely electromagnetic character cannot be maintained. The term "helicon" was originally coined by Aigrain ${ }^{25}$ in 1960 to describe the propagation of bounded right hand circularly polarized waves in a solid rod of sodium. The dispersion relation for the helicon wave is given by

$$
N^{2} \approx \frac{\omega_{p e}^{2}}{\omega \omega_{c e} \cos \theta},
$$

where $N$ is the parallel index of refraction, defined as $N=k_{\|} c / \omega, k_{\|}$is the wave number parallel to the magnetic field, $c$ is the speed of light, $\omega$ is the wave frequency, $\omega_{p e}$ is the electron plasma frequency, $\omega_{c e}$ is the electron cyclotron frequency and $\theta$ is the angle at which the wave propagates with respect to the magnetic field. One interesting feature of the helicon dispersion relation is that the maximum group velocity of the helicon waves is given by $(d \omega / d k)_{\max }=\omega / 4 \omega_{c e}{ }^{26}$ Therefore, high frequency helicon waves travel faster, arriving earlier than the low frequency waves emanating from the same source; giving rise to the same "whistling" effect characteristic of the unbounded whistler waves recorded in the early part of the $20^{\text {th }}$ century.

Since helicons are propagating whistler wave modes in an axially magnetized, finite diameter plasma column, the electric and magnetic fields of the helicon modes have radial, axial and azimuthal variations. They propagate in high density plasmas at relatively low magnetic fields and their operating frequency, $\omega$, relative to key plasma frequencies is characterized by $\omega_{L H} \ll \omega \ll \omega_{c e}$ and $\omega \omega_{c e} \ll \omega_{p e}^{2}$, where, $\omega_{L H}$ is the lower hybrid frequency, $\omega_{p e}=\left(n e^{2} / \varepsilon_{0} m\right)^{1 / 2}$ is the electron plasma frequency, $\omega_{c e}=e B / m_{e}$ is the electron cyclotron frequency and $n, e, \varepsilon_{0}, m$ and $B$ are the plasma density, elementary electron charge, dielectric permittivity of vacuum, electron mass and the uniform background magnetic field strength respectively. Including ion inertia effects, the lower hybrid frequency, $\omega_{L H}$, is given by

$$
\frac{1}{\omega_{L H}^{2}}=\frac{1}{\omega_{p i}^{2}+\omega_{c i}^{2}}+\frac{1}{\omega_{c e} \omega_{c i}},
$$


where $\omega_{p i}=\left(n Z^{2} e^{2} / \varepsilon_{0} M\right)^{1 / 2}$ is the ion plasma frequency and $\omega_{c i}=Z e b / M$ is the ion cyclotron frequency (with $Z e$ and $M$ being the ion charge and mass respectively). In high density plasmas, the first term is negligible and hence $\omega_{L H} \approx\left(\omega_{c e} \omega_{c i}\right)^{1 / 2}$.

The physical process responsible for efficient helicon source operation (very high plasma densities at relatively low temperatures given the if input power) have been extensively studied over a wide variety of operating regimes. Collisional processes, ${ }^{22,23}$ Landau damping, ${ }^{27,28}$ helicon wave penetration, ${ }^{29}$ antenna localized acceleration, ${ }^{30,31}$ mode conversion near the lower hybrid frequency, ${ }^{32}$ non linear trapping of fast electrons $^{33,34}$ and ion heating ${ }^{35}$ have all been considered as possible explanations for the helicon source's high efficiency in creating plasma.

Recent studies of helicon plasma sources have focused on both high efficiency operation and strong wave damping, neither of which is fully explained by either collisional or Landau damping processes. The possible role of a population of fast electrons, constituting a non-Maxwellian component of the electron distribution function, in ionizing the background gas in a helicon source is another important and related issue.

The plasma density and the parallel wave number obey a fixed relationship for a helicon wave for a given magnetic field strength ${ }^{36}$

$$
k=\sqrt{k_{\perp}^{2}+k_{\square}^{2}} \approx \frac{\omega}{k_{\square}} \frac{\omega_{p}^{2}}{\omega_{c e} c^{2}}=e \mu_{0} v_{p}\left(\frac{n}{B}\right),
$$

where $k$ is the wave number of the helicon wave, $\mu_{0}$ is the magnetic permeability in vacuum and $v_{p}=\omega / k_{\|}$is the helicon waves' phase velocity along the tube. Generally $k_{\perp}$ is fixed by the tube radius $a$, such that $J_{1}\left(k_{\perp} a\right)=0$ (where $J_{1}$ is the Bessel function of the first kind). It follows then that for a gas which has a peak in its ionization cross-section of $\sim 50 \mathrm{eV}$, such as Argon, matching the wave phase speed to electrons with kinetic energies at the peak of the ionization cross-section requires

$$
B / n=220 a, v_{p}=f . \lambda=4.19 \times 10^{6} \mathrm{~m}_{\mathrm{sec}}{ }^{-1} \text { and } f=32 / a \text {; }
$$


with $n$ in units of $10^{13} \mathrm{~cm}^{-3}$, the tube radius $a$ in $\mathrm{cm}$, and the driving frequency $f$ in $\mathrm{MHz}$. For the typical industrial frequency of $13.56 \mathrm{MHz}$, a tube of $\sim 5 \mathrm{~cm}$ diameter will require a magnetic field of $\sim 275 \mathrm{G}$ to generate plasma of density $\sim 5 \times 10^{12} \mathrm{~cm}^{-3}$. When these design targets are met in practice, the density performance is generally consistent with these simple predictions.

Even in the case of uniform magnetic fields, the mechanisms of plasma creation and loss in helicon sources are not completely understood. The axial profile of plasma density downstream of the antenna is sensitive to many operating parameters such as neutral pressure, antenna design, magnetic field strength etc. In the particular case of small diameter tubes, the axial density scale length is much shorter than the calculated damping distance and may be related to depletion of neutrals or formation of double layers. ${ }^{37}$

In the case of nonuniform magnetic fields, specifically an expanding magnetic field geometry, helicon sources have played a key role in recent studies of spontaneously forming double layers. Investigation of high-density, low temperature plasmas expanding into a vacuum or into a low density background plasma date back to the 1930s, when high velocity plasma jets were observed in low pressure dc discharges. ${ }^{38,39}$ Later experiments demonstrated acceleration of ions to supersonic speeds during plasma expansion. ${ }^{40,41,42}$ Detailed measurements of electron and ion velocity distribution functions during the expansion process in a pulsed plasma have been reported. ${ }^{43,44,45}$ The physics of expanding plasmas play a key role in understanding a wide range of phenomena: in the filling of the wake region behind objects moving supersonically through a plasma, ${ }^{46}$ in laser fusion experiments when the laser heated target material moves away from the target, ${ }^{47}$ and in the expansion of ionospheric plasma into the magnetosphere along the earth's magnetic field. ${ }^{48}$ Different laboratory experiments designed to study the details of expanding plasmas include the use of pulsed plasma sources, ${ }^{43,44}$ Q-machines with shaped magnetic fields, ${ }^{49,50}$ cathode-anode plasma sources, ${ }^{51}$ and triple plasma devices. ${ }^{52,53}$ 


\subsection{WVU Helicon Plasma Sources}

At present there are two independent, active helicon plasma sources at WVU. One is the HELIX-LEIA device (Fig. 2.1) and the other is CHEWIE (Fig. 2.7). While both machines share many common features of helicon sources and similar experimental geometry, there are key differences between the two sources. In the following sections, detailed descriptions are provided for each device. The experimental work related to Electrostatic Double Layers (EDLs) in expanding plasma was performed in the HELIXLEIA device while the measurements of ion velocity distribution function (IVDF) using continuous wave cavity ring down spectroscopy (CW-CRDS) were performed in CHEWIE.

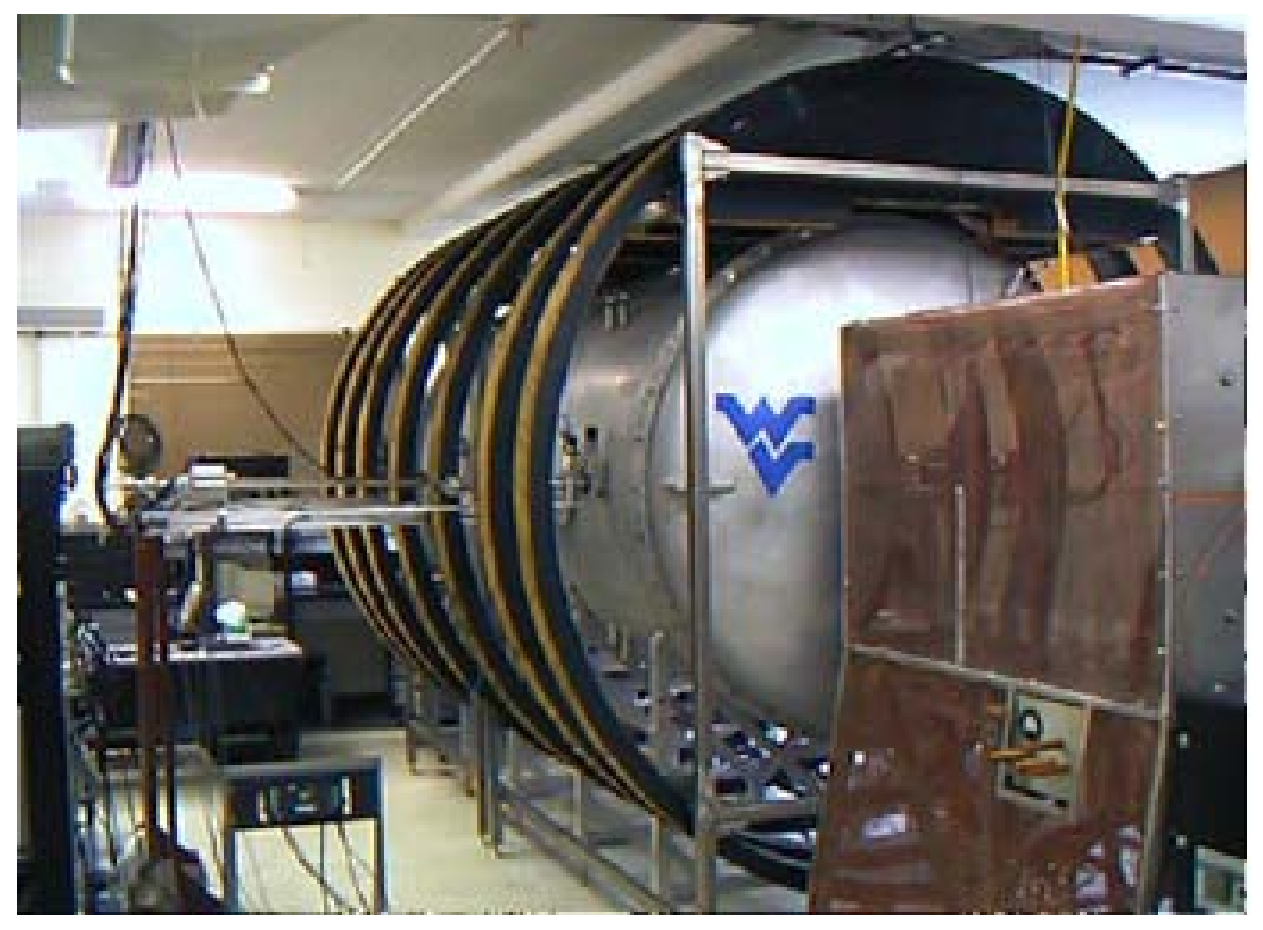

Figure 2.1. HELIX (foreground) and LEIA (large aluminum chamber). HELIX resides inside a Faraday cage for rf shielding. The large electromagnets surrounding LEIA are approximately $3 \mathrm{~m}$ in diameter. The figure is adapted from Ref. [57].

\subsubsection{HELIX - LEIA Experimental device}

This helicon plasma source consists of two regions: the small diameter HELIX (Hot hELIcon eXperiment) where the plasma is produced and the large expansion region 
LEIA (Large Experiment on Instabilities and Anisotropies). The plasma created in HELIX (Fig. 2.2) flows into the LEIA, which has a weaker magnetic field. The resultant high beta (particle pressure/magnetic field pressure i.e. $\beta=n k_{B} T \mu_{0} / B^{2}$ ) characteristic of LEIA plasma is ideally suited for laboratory investigations of both magnetospheric and heliospheric relevant experiments. The geometry of the magnetic field expansion region between HELIX and LEIA also enables the studies of spontaneous, current-free, electrostatic double layer formation at low neutral pressures. Detailed descriptions of the early development of the HELIX in WVU can be found in the dissertations of Kieter, ${ }^{54}$ Balkey ${ }^{55}$ and Kline. ${ }^{56}$ Details of more recent modifications to the HELIX source and several calibration measurements for the source parameters are found in the dissertations of Sun, ${ }^{57}$ Keesee, ${ }^{58}$ Hardin $^{59}$ and Biloiu. ${ }^{60}$

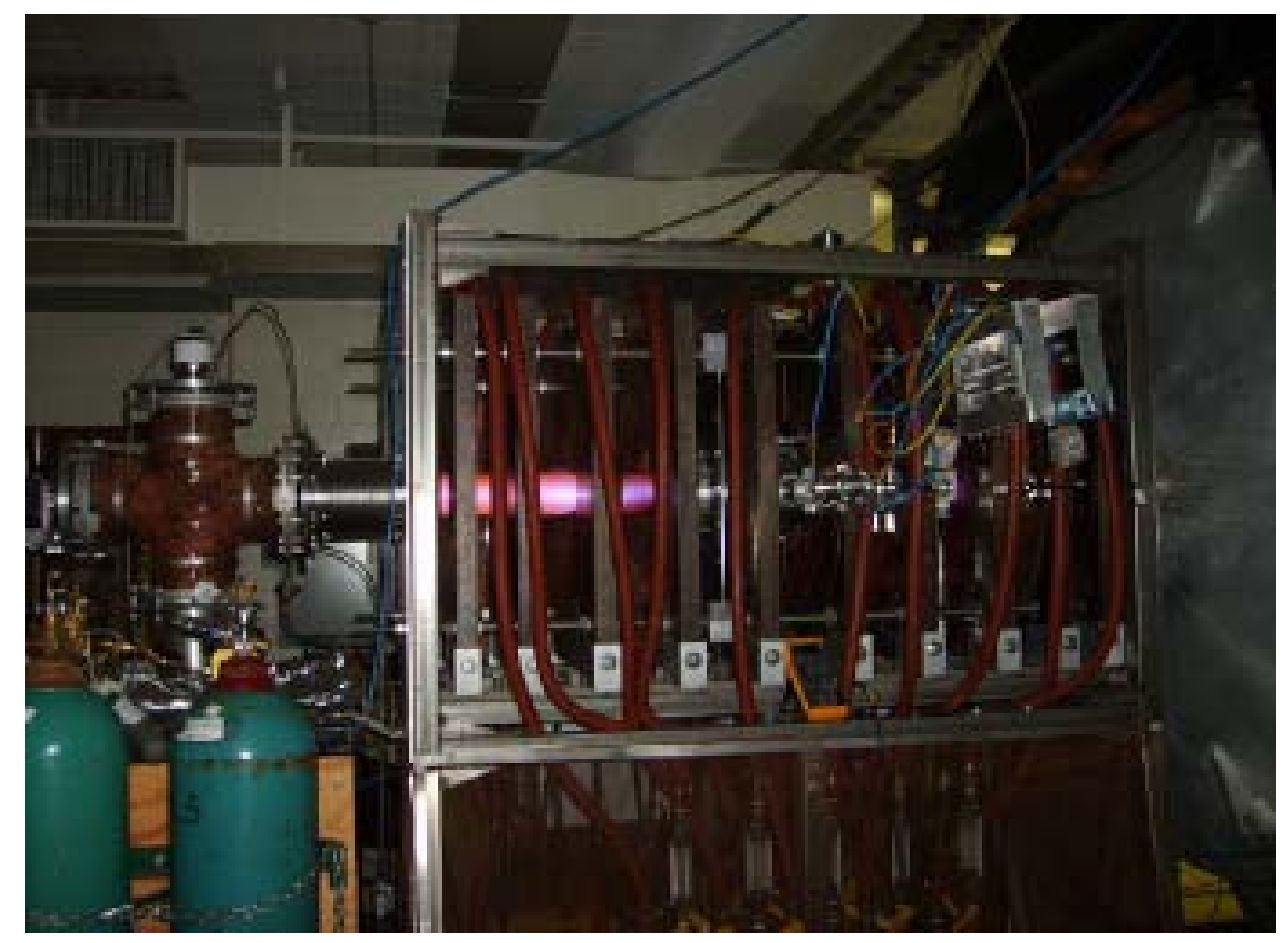

Figure 2.2. The HELIX helicon plasma source. On the far left is the four way cross with the mating flange connected to one of the pumping stations. The antenna is tightly wrapped around the Pyrex tube at about $37 \mathrm{~cm}$ from the closed end. In the middle, the Pyrex tube is connected to the 6' ID stainless steel chamber. In the far right is the connection of the HELIX to the large aluminum expansion chamber LEIA. The rf shielding is taken off to show the details. The figure is adapted from Ref. [60]. 


\subsubsection{Plasma Chamber}

The HELIX-LEIA system (Fig. 2.1) is shown schematically in Fig. 2.3. The HELIX vacuum chamber is a 24” long, Pyrex tube 4” in diameter connected to a 36” long, 6” inner diameter stainless steel chamber. The stainless steel chamber has one set of four 6" Conflat ${ }^{\mathrm{TM}}$ crossing ports in the center of the chamber and four sets of four 2.75" Conflat $^{\mathrm{TM}}$ crossing ports. The 2.75" Conflat ${ }^{\mathrm{TM}}$ crossing ports are spaced evenly on either side of the set of the 6" Conflat ${ }^{\mathrm{TM}}$ crossing ports. The left end of the 6" ID stainless steel chamber is directly connected to the LEIA chamber which has an inner diameter of $1.8 \mathrm{~m}$ and a length of $4.4 \mathrm{~m}$. The far end of the LEIA chamber, away from HELIX, is connected to a turbo-molecular pumping station. The other end of the HELIX chamber, opposite to LEIA, is connected to a glass cross. The other three legs of the cross are terminated with another turbo-molecular pumping station, an ion gauge and a 12” stainless steel flange fitted with a 4" viewport, respectively. LEIA has several accessible ports on either side used for the injection of our scanning internal probes (see Chapter 3)

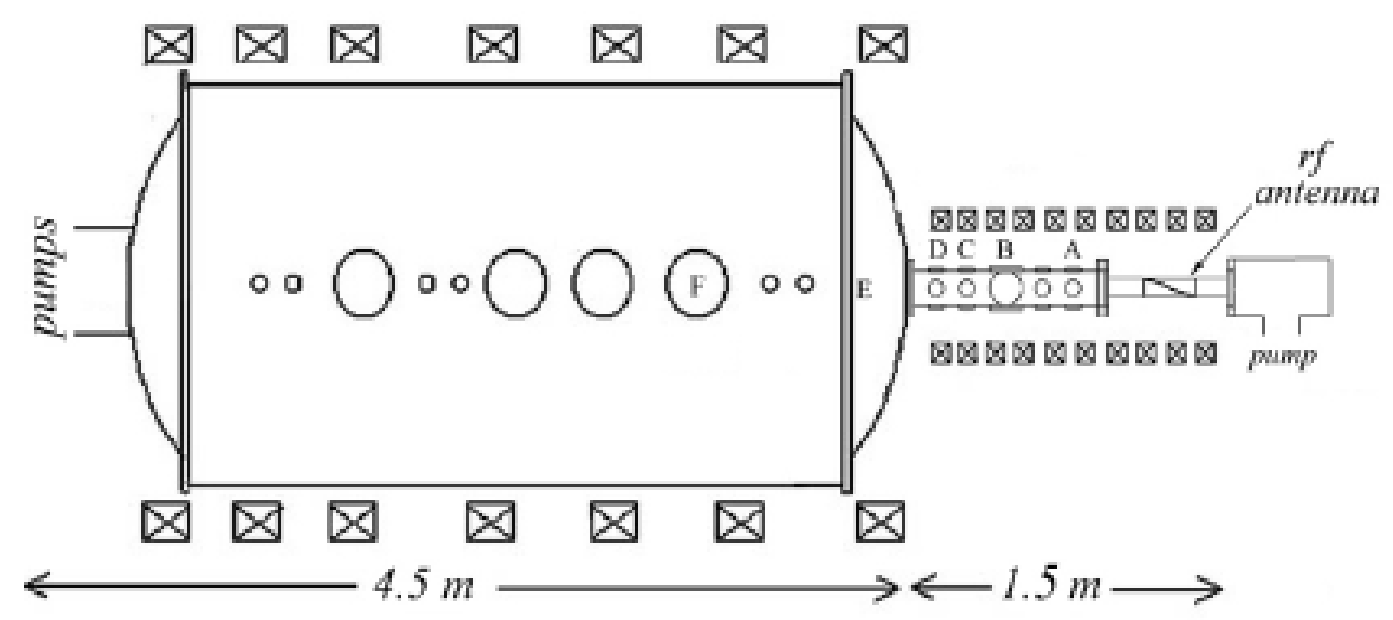

Figure 2.3. Schematic (side view) of the HELIX-LEIA plasma chamber along with the locations of the various diagnostics (more details in Chapter (5)).The figure is adapted from Ref. [57].

\subsubsection{Vacuum system}

The vacuum pressure in the chamber is maintained by a set of three turbomolecular drag pumps, each backed by a diaphragm roughing pump. A single Balzers 
TMU 520 turbo-molecular drag pump is connected to one branch of the glass cross at the end of HELIX. A Balzers MD 4T diaphragm roughing pump is attached to the turbo pump. A MDC GV-4000M-P 6" ID gate valve is located between the turbo pump and the glass tee. Two Pfeiffer TMU 1600 turbo-molecular pumps are connected to the far end of the LEIA chamber. Each of the turbo pumps is backed by a Pfeiffer MD-8 diaphragm roughing pump. Two MDC GV-8000V-P 10" ID gate valves separate the turbo pumps from the LEIA chamber. Each of the LEIA pumps can be run at a rotation frequency of either $400 \mathrm{rpm}$ or $600 \mathrm{rpm}$, allowing for three pumping rates in the expansion chamber. There is a slight pressure gradient in the HELIX along the source axis, while the pressure is nearly constant in LEIA and almost an order of magnitude smaller than the pressure in HELIX. The device can run with the front (near HELIX) gate valve either shut or open.

The three pumps maintain a base pressure on the order of $10^{-7}$ Torr. An interlock system is used to shut the gate valves in the event of a high pressure event (from a leak in the chamber or otherwise) or loss of power. The pressure is measured by two Balzers PKR250 full range pressure gauges and a Baratron ${ }^{\circledR}$ capacitance manometer. The Balzers gauges achieve full range by combining a Pirani gauge for pressures above $10^{-2}$ Torr and a cold cathode gauge for pressures below $10^{-2}$ Torr. One advantage of the Baratron ${ }^{\circledR}$ gauge is that the measurement is independent of the gas species, while the Balzers gauges require correction depending on the gas being used. The pressure gauge for the HELIX pumping station is located on one branch of the glass cross at one end. The gauge for the LEIA pumping station is located on LEIA, toward the pumping station end of the chamber. The Baratron ${ }^{\circledR}$ capacitance manometer pressure gauge is located 35 $\mathrm{cm}$ downstream of the antenna, approximately around the middle of HELIX.

The gas (or mixture of gases) flow rate is regulated by two MKS1179 mass flow valves controlled by a PR-4000 power supply. The gases used for the plasma discharges can be introduced at two locations in HELIX. One location is next to the Balzers pressure gauge on the glass cross. In this experiment, this "end gas feed" location is used. The other location for gas introduction is at a 2.75" cross port on the stainless steel portion of the HELIX chamber closest to the antenna. This gas feed allows for a more direct gas flow into the plasma chamber near the antenna instead of relying on diffusion 
overcoming the pumping at the "end gas feed" location. Neutral operating pressures of 0.1 to 100 mTorr are typically achieved in HELIX.

\subsubsection{Magnetic Field}

Ten water-cooled electromagnets, donated to WVU by the Max Planck Institüt in Garching, Germany, produce a steady state, almost uniform, axial magnetic field of 01300 Gauss in HELIX. Each magnet has 46 internal copper windings with a resistance of $17 \mathrm{~m} \Omega$ and an inductance of $1.2 \mathrm{mH}$. The axial position of each magnet is adjustable along a set of rails. Two Xantrex XFR power supplies connected in parallel provide a total current of up to 400 Amperes to the electromagnets. These Xantrex power supplies replace the original MacroAmp power supply which was found to have significant current fluctuations. ${ }^{56}$

The LEIA magnetic field is produced by a set of seven custom-built, 9' diameter water cooled electro-magnets. Each magnet consists of five sets of aluminum tubing wound into five two-coil "pancakes" of four layers each, for a total of 40 turns per magnet. The 0.5 ” x 0.5 ” square tubing is hollow and wrapped in an insulating paper. The magnets are water-cooled by a closed loop system maintained with a Neslab NX-300 chiller. These magnets are upgraded versions of the original 20 turn coils used previously

on LEIA. $^{55,56}$ In these new electromagnets, the larger size aluminum tubing (and therefore lower electrical resistance) and increased number of turns provide a factor of two increase in the generated magnetic field strength for the same input power as in the original electromagnets. Moreover, the inner hole of the new tubing is circular as opposed to being rectangular, thus allowing for better attachment of the water connections. A LEIA magnetic field of 0-130 Gauss can be produced using a 200 Amperes DC EMHP power supply. 


\section{LEIA magnets}

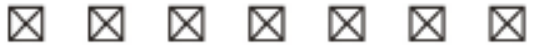

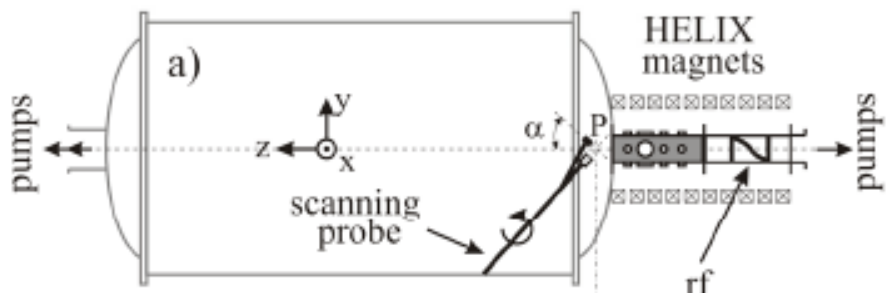

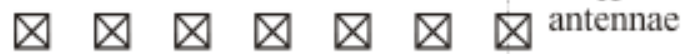
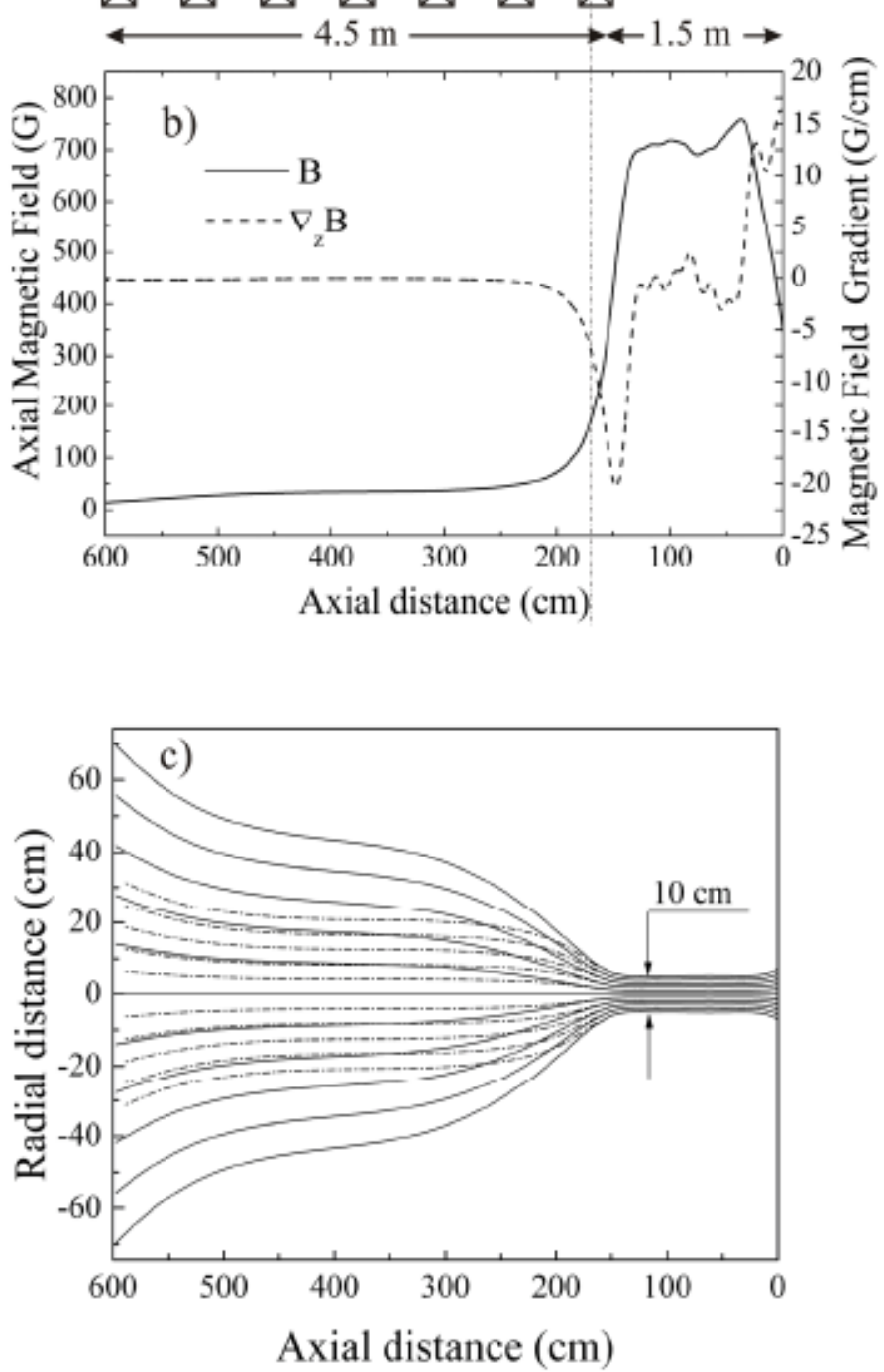

Figure 2.4. (a) HELIX-LEIA device drawn to scale with the magnetic field profiles shown. (b) Magnetic field strength and magnetic field gradient versus axial position over the entire length of the HELIX-LEIA device. (c) Contours of constant magnetic field flux showing the increased divergence that results when the magnetic field in the LEIA decreases from $70 \mathrm{G}$ (dash-dot line) to $14 \mathrm{G}$ (solid line), for a constant $600 \mathrm{G}$ magnetic field in HELIX. The figure is adapted from Ref. [57]. 
Under typical operating conditions, in the connection region between the helicon source HELIX and the expansion chamber LEIA, there is an axial magnetic field gradient of approximately 10 Gauss/cm over a distance of $70 \mathrm{~cm}$. Fig. 2.4(b) shows the on-axis magnetic field strength and its gradient in the HELIX-LEIA combined system as calculated with a two dimensional numerical model that was validated with point measurements along the system axis. The spatial variation of the contour lines of constant magnetic flux (flux tubes) are also shown in Fig. 2.4(c) for a constant magnetic field strength of $B_{H}=600 \mathrm{G}$ in HELIX and for two different but individually constant magnetic field strengths in LEIA: $B_{L}=70 \mathrm{G}$ (dash-dot line) and $B_{L}=14 \mathrm{G}$ (solid line).

\subsubsection{Rf antenna and Matching Network}

The plasma is created in HELIX by a $19 \mathrm{~cm}$ long half wave, right-handed $(\mathrm{m}=1)$ helical antenna (Fig. 2.5) wrapped around the outside of the Pyrex vacuum chamber, at approximately $37 \mathrm{~cm}$ from the closed end of HELIX. A detailed description of the matching network and the antenna can be found in the dissertations of Balkey ${ }^{55}$ and Sun. ${ }^{57}$ The rf system for HELIX consists of a $50 \mathrm{MHz}$ Wavetek model-80 function generator that supplies the rf signal to an ENI 1000, a $30 \mathrm{~dB}$ amplifier which provides up to $2 \mathrm{~kW}$ of power over the frequency range 6-18 MHz. The rf power is transmitted from the amplifier to the source through a high frequency co-axial cable and a $\pi$-type matching network to match the $50 \Omega$ output impedance of the amplifier to the antenna/matching network system. The matching network consists of one load and three tuning capacitors. The load capacitor is a large, tunable, Jennings high voltage vacuum capacitor with a range of $20-2000 \mathrm{pF}$. The tuning capacitors (two with a range of $4-250 \mathrm{pF}$ and one with a range of $5-500 \mathrm{pF}$ ) are all Jennings high voltage tunable capacitors. All the capacitors are connected by sheets of copper, which are connected to the antenna by rods of silver-plated copper. The three tuning capacitors are in parallel to each other and in series with the antenna. The whole combination is then in parallel with the load capacitor, as shown in the schematic in Fig. 2.6. To maximize the antenna coupling efficiency, the real impedance of the matching network must equal the output impedance of the amplifier, $\mathrm{R}_{0}=50 \Omega$, and the imaginary part of the combined matching network-antenna circuit must be zero. 

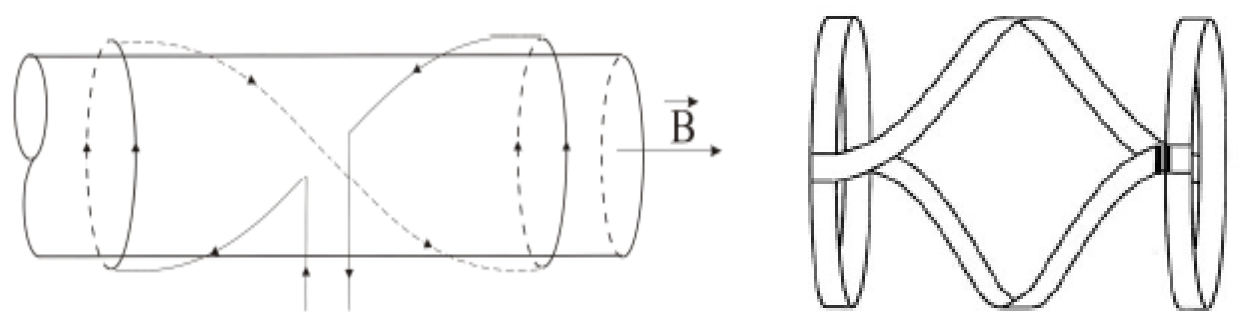

Figure 2.5. Diagram of $m=+1$ helical antenna and the actual design of the antenna ${ }^{61}$. The figure is adapted from Ref. [57].

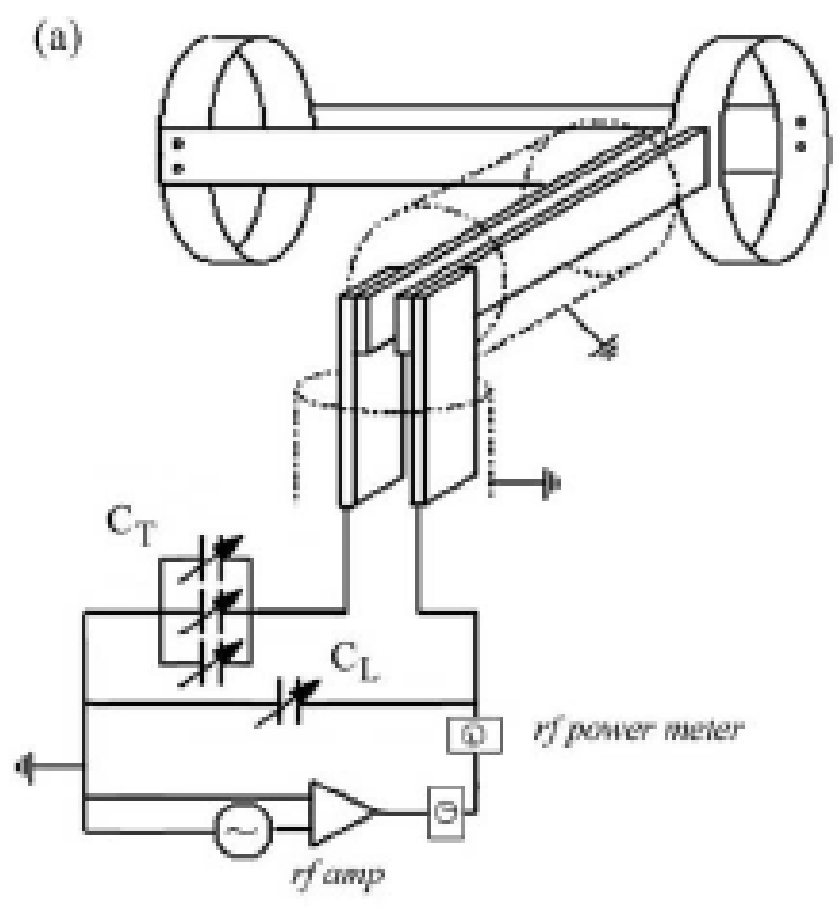

Figure 2.6. Matching circuit for the helicon antenna for HELIX. $C_{L}$ is the load capacitor and $C_{T}$ are the three tuning capacitors. The figure is adapted from Ref. [57].

Denoting the load capacitance by $C_{L}$ and the total tuning capacitance by $C_{T}$, the real resistance of the antenna by $R$, and the reactance $\omega L$, by $X$, $C h e n^{62}$ calculated the required load and tuning capacitances for an inductive load to be

$$
C_{L}=\frac{1}{2 \omega R}\left[1-\left(1-\frac{2 R}{R_{0}}\right)\right]^{1 / 2}
$$


and

$$
C_{T}=\left[\omega X-\frac{1-R / R_{0}}{C_{L}}\right]^{-1} .
$$

After the discharge is initiated, the effect of the inductive load of the plasma on the antenna has to be considered. For a typical helicon plasma source in the "inductive" or "helicon" mode, the above equation becomes

$$
C_{T}^{-1}=\omega^{2} L-\frac{\left(1-\frac{R}{R_{0}}\right)}{C_{L}},
$$

where $L$ is the total inductance in the antenna portion of the circuit. 


\subsubsection{HELIX-LEIA Plasma Parameters}

Here we report typical plasma parameters in both HELIX and LEIA for argon plasma.

\begin{tabular}{|c|c|c|}
\hline Parameter (units) & HELIX & LEIA \\
\hline$B$ (Gauss) & $500-1200$ & $5-70$ \\
\hline$p_{\text {neutral }}$ (mTorr) & $1.5-10$ & $0.15-1$ \\
\hline$n\left(\mathrm{x} 10^{12} \mathrm{~cm}^{-3}\right)$ & $0.1-10$ & $0.001-0.1$ \\
\hline$T_{e}(\mathrm{eV})$ & $4-12$ & $2-8$ \\
\hline$T_{i}(\mathrm{eV})$ & $<1$ & $<1$ \\
\hline$\lambda_{\text {Debye }}(\mathrm{cm})$ & $5 \times 10^{-3}-8 \times 10^{-4}$ & $3 \times 10^{-2}-6.5 \times 10^{-2}$ \\
\hline$r_{e}(\mathrm{~cm})$ & $4 \times 10^{-3}-2 \times 10^{-2}$ & $5 \times 10^{-2}-1.5$ \\
\hline$r_{i}(\mathrm{~cm})$ & $2 \times 10^{-1}-6 \times 10^{-1}$ & $4-60$ \\
\hline$\omega_{p e}\left(10^{10} \mathrm{rad} / \mathrm{s}\right)$ & $1.8-18$ & $0.18-18$ \\
\hline$\omega_{c e}\left(10^{9} \mathrm{rad} / \mathrm{s}\right)$ & $5.2-34$ & $52-340$ \\
\hline$\omega_{c i}\left(10^{6} \mathrm{rad} / \mathrm{s}\right)$ & $0.12-0.3$ & $1.2 \times 10^{-3}-1.6 \times 10^{-2}$ \\
\hline
\end{tabular}




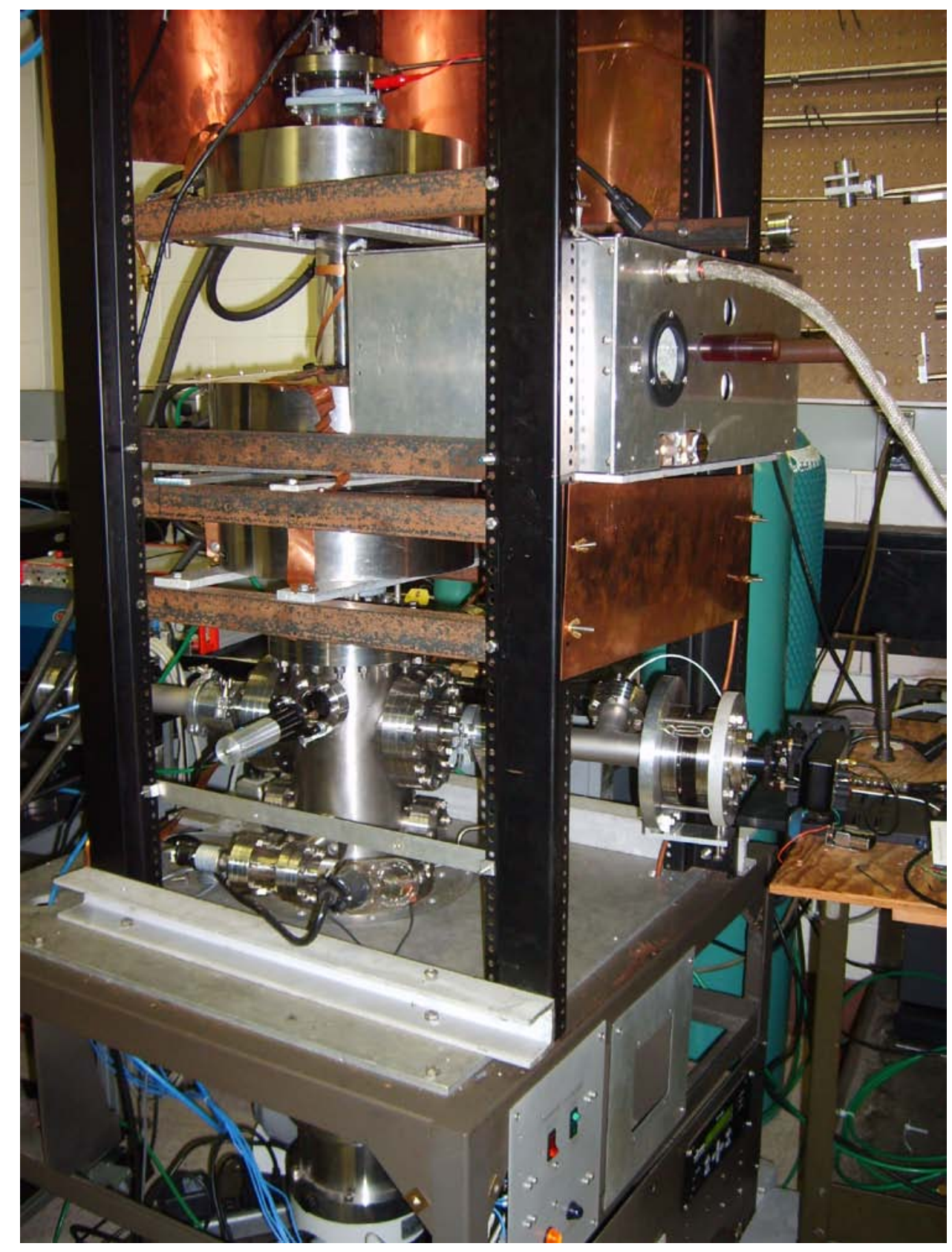

Figure 2.7. Photo of CHEWIE, with the copper rf shielding taken off.

\subsubsection{CHEWIE Experimental device}

The second helicon source in operation at WVU is CHEWIE (Compact HElicon Waves and Instabilities Experiment). The smaller diameter source chamber opens into a larger diameter expansion chamber similar to the HELIX-LEIA device. One difference is 
that CHEWIE (Fig. 2.7) is a vertical device. The plasma is produced in the source chamber and then flows down to the expansion chamber.

Their relatively high plasma densities and the possibility to either supply an independent voltage to the sample substrate or to allow the sample holder to electrically float are characteristics that make helicon sources particularly attractive as plasma processing sources. In a plasma processing configuration, the sample to be processed is placed in the expansion chamber connected to the helicon source. The plasma expands from the strong magnetic field region of the helicon source into the weaker magnetic field of the expansion chamber. For material processing applications involving deposition, surface modification or etching, control of the ion temperature, ion speed, plasma density and uniformity in the expanding plasma is of utmost importance. It has been shown that in the expansion chamber, charge exchange collisions associated with plasma expansion reduce the average energy of the ions impinging on the target. Helicon plasma sources have been used to generate uniform plasma fluxes with a high plasma density but with reduced ion energies at the substrate location in the diffusion chamber. ${ }^{63,64,65}$ Arrays of compact helicon plasma sources have been shown to produce uniform plasmas over a large surface area. ${ }^{66}$ For plasma etching, high etching rates $(1.5 \mu \mathrm{m} / \mathrm{min}$. $)$ with minimal anisotropy (0.97) were obtained with expanding $\mathrm{SF}_{6}$ helicon plasma. ${ }^{67}$ Better control of ion flow speed and ion temperature and an improved understanding of charge exchange collisions in the expansion region are needed for helicon sources to become competitive with more technologically mature, commercially available, plasma based, etching systems.

The CHEWIE experiment was specifically designed to explore the properties of helicon sources in a plasma processing relevant geometry. For the schematic details of the device, see Fig. 2.8. CHEWIE also serves the dual purpose of validating and testing different diagnostics before implementation in HELIX-LEIA. The vertical geometry allows the study of the effects of downward flowing plasma on material substrates. Being vertical, CHEWIE occupies much less laboratory space and is easily portable. 


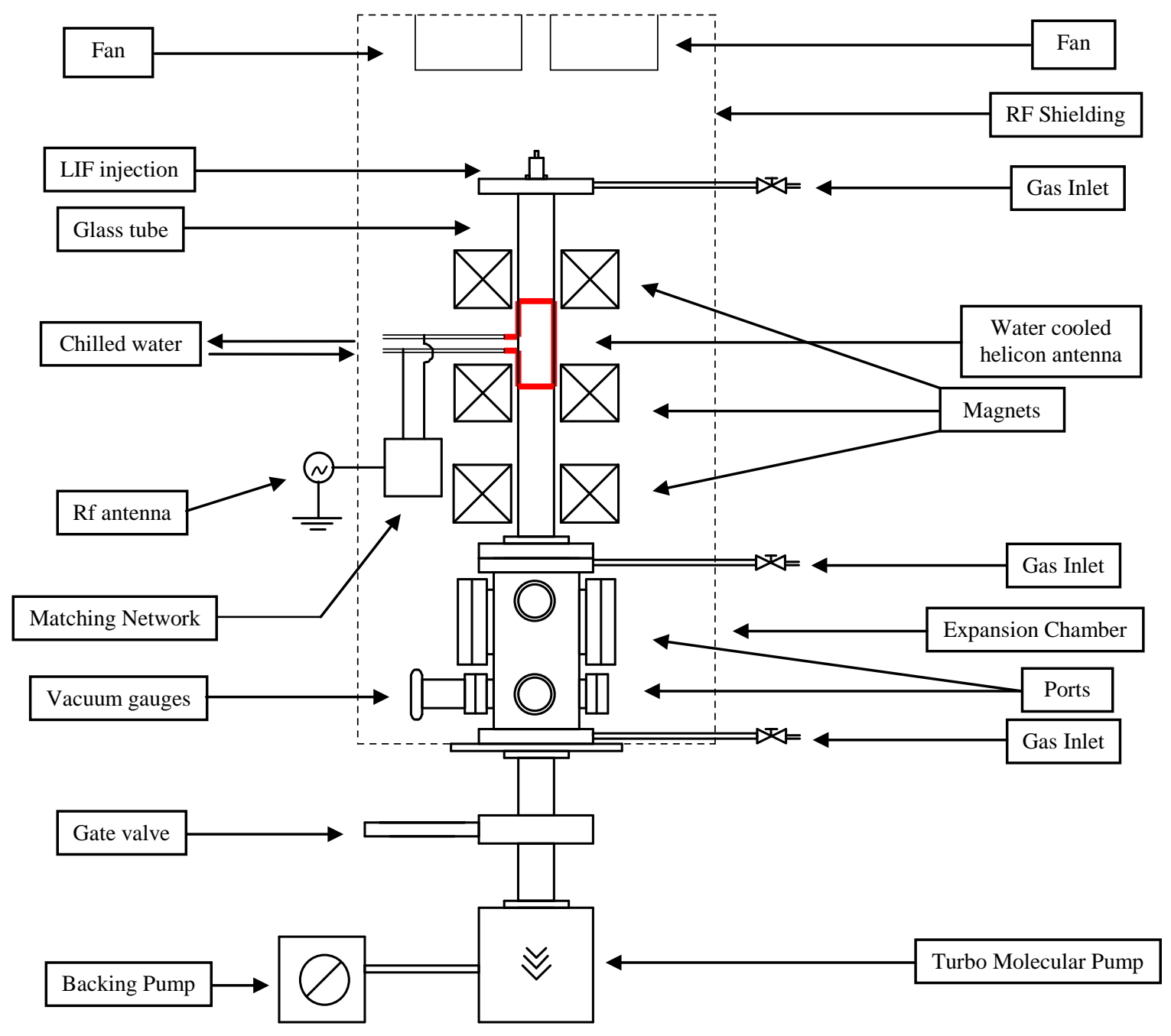

Figure 2.8. Schematic diagram showing the full details of the CHEWIE system.

\subsubsection{Plasma Chamber}

A less complicated schematic of the CHEWIE plasma chamber is shown in Fig. 2.9. The source chamber is a Pyrex glass tube 24” long and 3" in diameter. CHEWIE is air cooled by two fans placed above the chamber. At the top, the source chamber is closed by an adapter with a 2.75" Conflat ${ }^{\mathrm{TM}}$ glass window on which are mounted the LIF injection optics. The top of the chamber is defined as the $z=0$ position in CHEWIE. The other end of the glass tube is connected to the expansion chamber; $30 \mathrm{~cm}$ in length and 15 $\mathrm{cm}$ in diameter. The expansion chamber has several ports which are used for diagnostic access or gauges to monitor the chamber pressure. On the uppermost portion of the expansion chamber, two 2.75" ports (at position A in Fig. 2.9) serve as the rf- 
compensated Langmuir probe injection port and to hold the assembly for the LIF collection optics, respectively. A little below, there are two oppositely placed 6” ports (at position BB' in Fig. 2.9) which serve as the accessible ports for attaching the mirror holders for CRDS. Further down along the z-axis there are four symmetrically placed 2.75” ports (at position C in Fig. 2.9) which are used for other diagnostics or pressure gauges.

\subsubsection{Vacuum system}

The chamber is connected to a turbo molecular pump (TMU 520) at the bottom end, which is then backed by an XtraDry 150-2 Dry piston roughing pump. The base pressure achieved in the chamber using this combination is $\sim 10^{-7}$ Torr. The gas flow in the chamber is regulated by a MKS PR4000 power supply and a MKS 1179A mass flow controller. There are three possible places where the gas can be fed into the device: at the top of the source chamber, at the junction of the source and expansion chambers, and at the bottom of the expansion chamber (positions 1, 2 and 3 respectively in Fig. 2.9). The lowest achievable gas injection rate into the chamber is $11 \mathrm{sccm}$ (standard cubic centimeters per minute), which correspond to a pressure of $\sim 2.7$ mTorr. The limitation is due to the size of the aperture of the mass flow controller.

The pressure in the chamber was measured by a Kurt J. Lesker 4500 ion gauge controller which used a thermocouple and an ion gauge. The thermocouple gauge measured in a pressure range of 1 Torr to $1 \mathrm{mTorr}$, while the range of pressure measured by the ion gauge is from 1 mTorr down to $10^{-10}$ Torr. The filament in the ion gauge does not operate at pressures higher than 1 mTorr. 


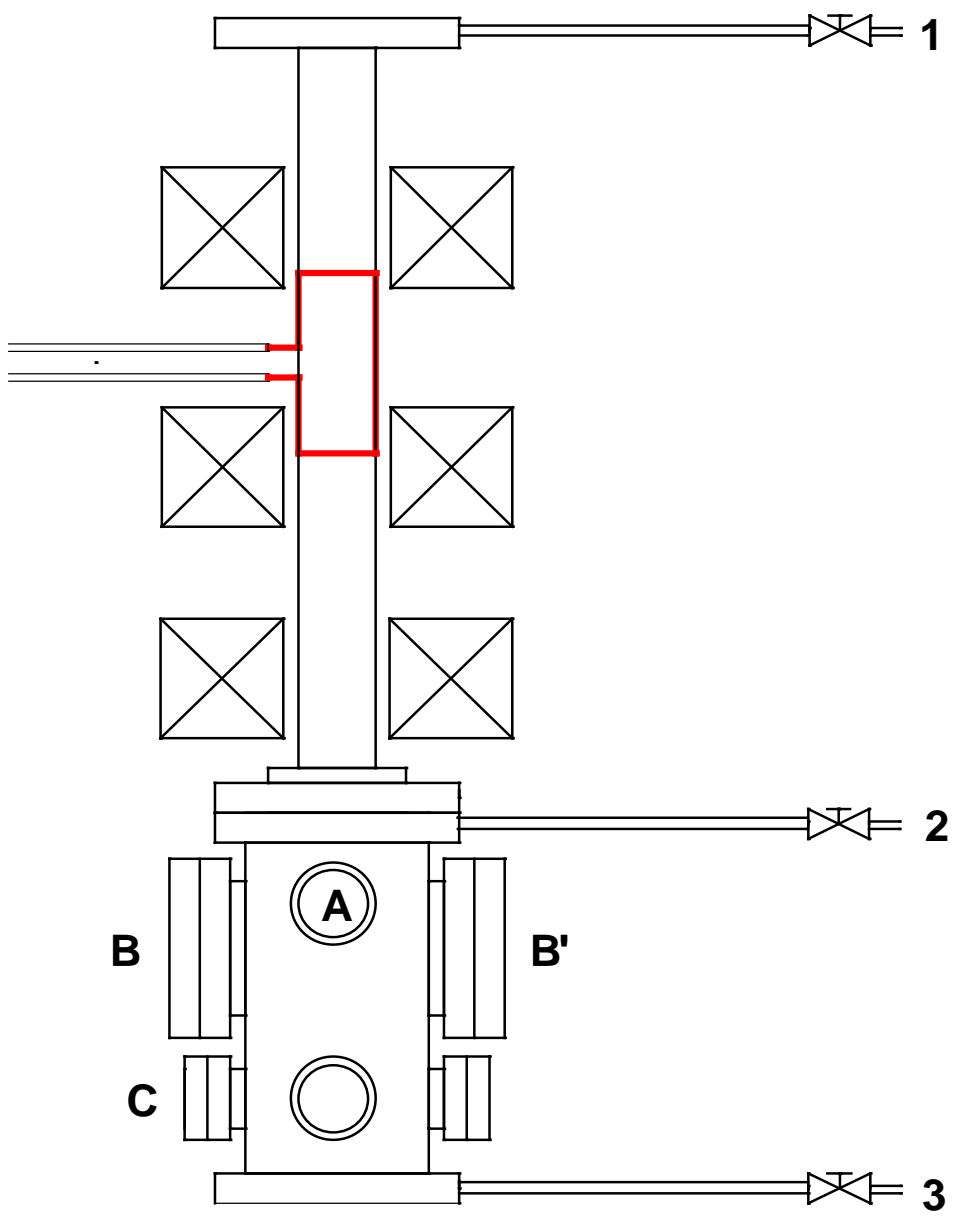

Figure 2.9. Schematic diagram of the vacuum chamber of CHEWIE. The positions of the gas feeds, the helicon antenna and the magnets are also shown.

\subsubsection{Magnetic Field}

The magnetic field for CHEWIE is provided by three water cooled electromagnets placed around the source chamber. Each magnet is $10 \mathrm{~cm}$ in height and has an inner radius of $5 \mathrm{~cm}$ and outer radius of $17 \mathrm{~cm}$. The three magnets were placed between $10.5 \mathrm{~cm}$ to $20.5 \mathrm{~cm}, 32.8 \mathrm{~cm}$ to $42.8 \mathrm{~cm}$ and $47.3 \mathrm{~cm}$ to $57.3 \mathrm{~cm}$. The magnetic field profiles for some values of current flowing through the magnets are shown in Fig. 2.10. An EMS-Power Supply provides the current for the electromagnets. The maximum rating of the power supply is $30 \mathrm{~A}$ at $200 \mathrm{~V}$. Typically a magnetic field strength of 1 kGauss was used (achieved at 10 Amps. of current). The magnets were water cooled by an Accurate Gas Controller Systems chiller Model 708. 


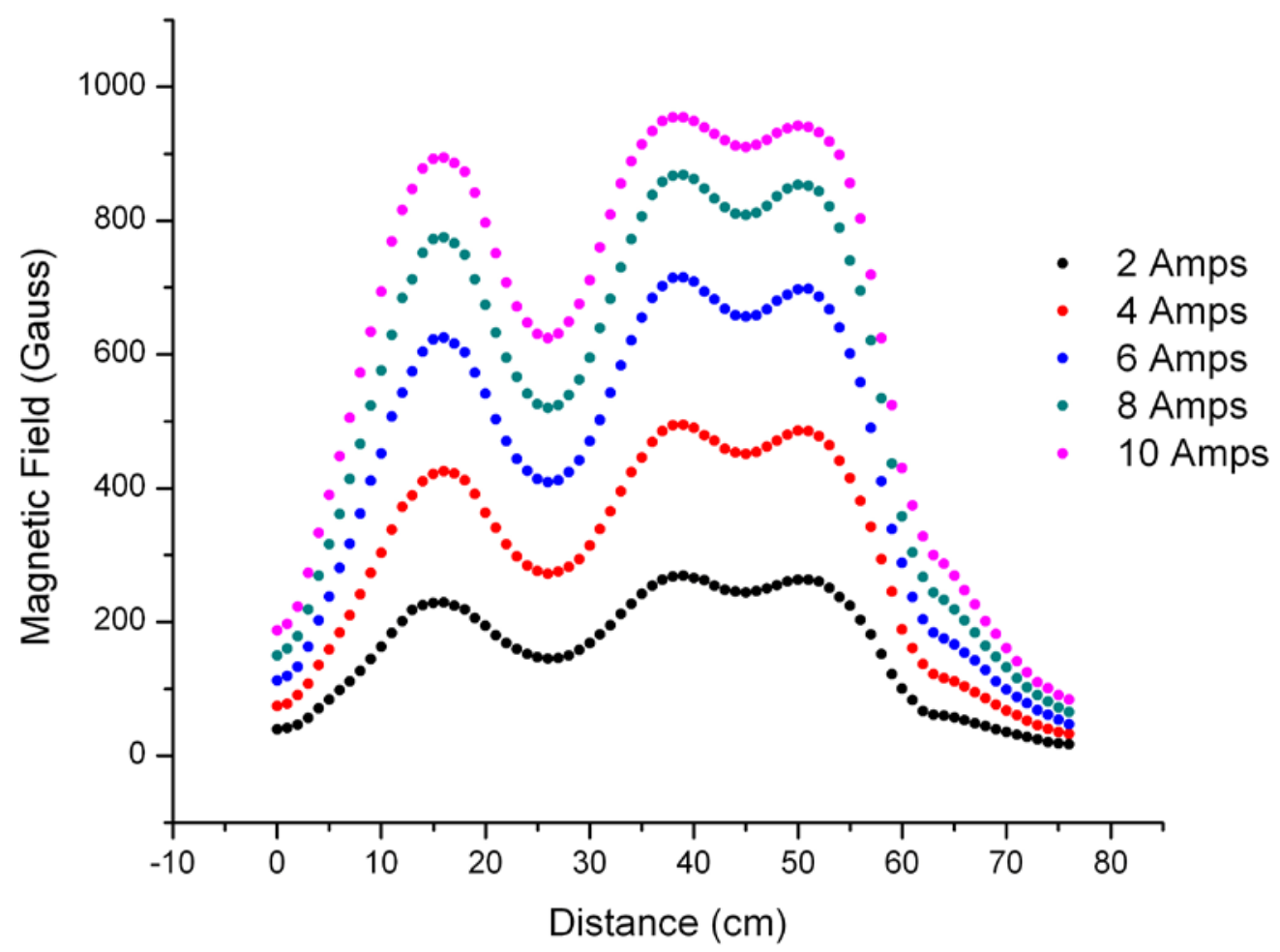

Fig. 2.10. Magnetic field profiles in CHEWIE for some values of current in the magnets.

\subsubsection{Rf antenna and matching network}

The plasma is created in CHEWIE by a $12.5 \mathrm{~cm}$ long $m=+1$ helical antenna (Fig. 2.11) wrapped around the outside of the Pyrex vacuum chamber; starting at approximately $22 \mathrm{~cm}$ from the closed end of the source chamber. The antenna was connected to the capacitors of the matching network by copper strips instead of wires to effectively reduce impedance and hence loss of rf power. A fan was used to keep the antenna and the surrounding Pyrex glass cool during plasma production. Copper sheets were used between the antenna and the bottom electromagnet to reduce the effective rf coupling from the antenna to the electromagnet. 


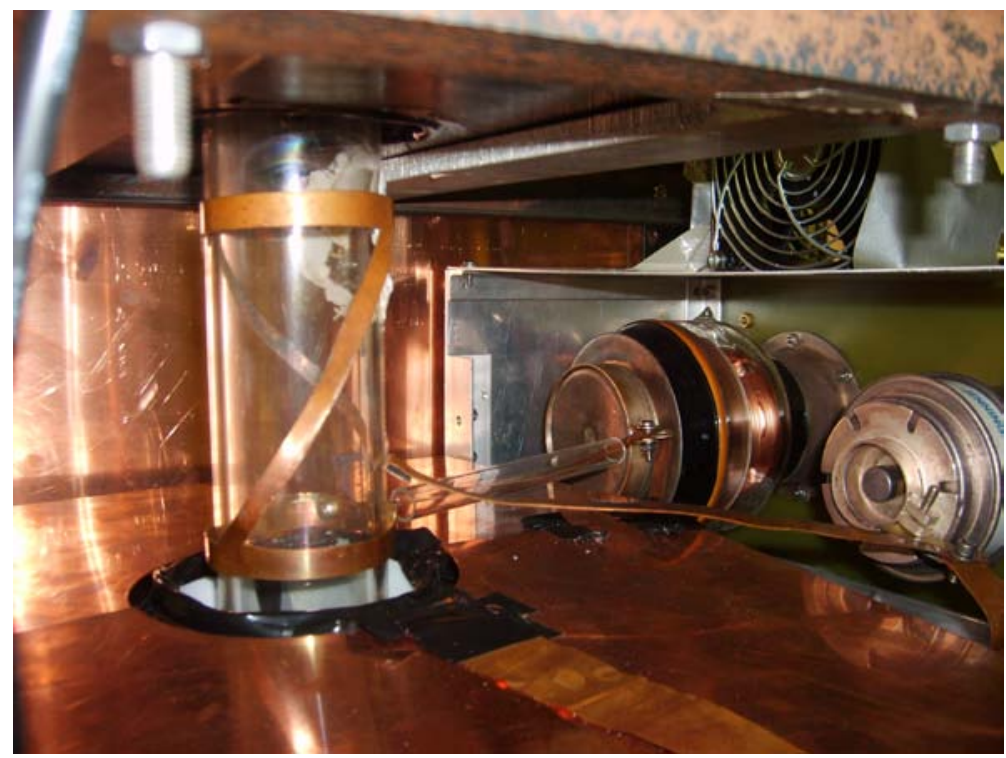

Figure 2.11. Picture of $m=+1$ helical antenna system used in CHEWIE. Also shown are the capacitors in the matching box and the fan used to keep the antenna cool during plasma production. The copper sheet at the bottom is to reduce rf coupling from the antenna to the electromagnets. 


\subsubsection{CHEWIE Plasma Parameters}

Here we report typical plasma parameters in CHEWIE for argon plasma.

\begin{tabular}{|c|c|}
\hline Parameter (units) & CHEWIE \\
\hline$B$ (Gauss) & $400-1000$ \\
\hline$p_{\text {neutral }}$ (mTorr) & $3-10$ \\
\hline$n\left(\mathrm{x} 10^{10} \mathrm{~cm}^{-3}\right)$ & $1-50$ \\
\hline$T_{e}(\mathrm{eV})$ & $3-8$ \\
\hline$T_{i}(\mathrm{eV})$ & 0.2 \\
\hline$\lambda_{\text {Debye }}(\mathrm{cm})$ & $1.8 \times 10^{-3}-2.1 \times 10^{-2}$ \\
\hline$r_{e}(\mathrm{~cm})$ & $4 \times 10^{-3}-1.7 \times 10^{-2}$ \\
\hline$r_{i}(\mathrm{~cm})$ & $2.9 \times 10^{-1}-7.2 \times 10^{-1}$ \\
\hline$\omega_{p e}\left(10^{10} \mathrm{rad} / \mathrm{s}\right)$ & $0.5-4$ \\
\hline$\omega_{c e}\left(10^{9} \mathrm{rad} / \mathrm{s}\right)$ & $7-18$ \\
\hline$\omega_{c i}\left(10^{5} \mathrm{rad} / \mathrm{s}\right)$ & $0.9-2.4$ \\
\hline
\end{tabular}




\section{Chapter 2 References}

${ }^{1}$ R. Bowers, C. Legendy and F. E. Rose, Phys. Rev. Lett. 7, 339 (1961)

${ }^{2}$ G. N. Harding and P. C. Thonemann, Proc. Phys. Soc. 85, 317 (1965)

${ }^{3}$ L. C. Woods, J. Fluid Mech. 18, 401 (1964)

${ }^{4}$ J. P. Klozenberg, B. McNamara and P. C. Thonemann, J. Fluid Mech. 21, 545 (1965)

${ }^{5}$ B. J. Davies and P. J. Christiansen, Plasma Phys. 11, 987 (1969)

${ }^{6}$ R. W. Boswell, Plasma Phys. Controlled Fusion 26, 1147 (1970)

${ }^{7}$ R. W. Boswell, A Study of Wave in Gaseous Plasma, Ph. D. Thesis, Flinders University, Adelaida, Australia (1974)

${ }^{8}$ R. W. Boswell, R. K. Porteus, A. Bouchoule and P. Ranson, Phys. Lett. A 91, 163 (1982)

${ }^{9}$ R. W. Boswell and D. Henry, Appl. Phys. Lett. 47, 1095 (1985)

${ }^{10}$ R. W. Boswell and R. K. Porteus, J. Appl. Physics. 62, 3123 (1987)

${ }^{11}$ J. P. Squire, F. R. Chang-Diaz, T. W. Glover, V. T. Jacobson, D. G. Chavers, R. D. Bengtson, E. A. Bering III, R. W. Boswell, R. H. Goulding and M. Light, Fusion Sci. Technol. 43, 111 (2003)

${ }^{12}$ R. W. Boswell, O. Sutherland, C. Charles, J. P. Squire, F. R. Chang-Diaz, T. W. Glover, V. T. Jacobson, D. G. Chavers, R. D. Bengtson, E. A. Bering, R. H. Goulding and M. Light, Phys. Plasmas 11, 5125 (2004)

${ }^{13}$ X. Sun, C. Biloiu, R. Hardin and E. E. Scime, Plasma Sources Sci. Technol. 13, 359 (2004)

${ }^{14}$ R. Winglee, T. Ziemba, L. Giersch, J. Prager, J. Carscadden and B. R. Roberson, Phys. Plasmas 14, 63501 (2007)

${ }^{15}$ G. W. Gibson Jr. and D. J Hemker, Semiconductor International 19, 193 (1996)

${ }^{16}$ S. Kimura and H. Ikoma, J. Appl. Phys. 85, 551 (1999)

${ }^{17}$ E. E. Scime, P. A. Keiter, M. M. Balkey, R. F. Boivin, J. L. Kline, M. Blackburn and S. P. Gary, Phys. Plasmas 7, 2157 (2000)

${ }^{18}$ J. Hanna and C. Watts, Phys. Plasmas 8, 4251 (2001)

${ }^{19}$ K. Itoh, S. Itoh, P. H. Diamond, T. S. Hahm, A. Fujisawa, G. R. Tynan, M. Yagi and Y. Nagashima, Phys. Plasmas 13, 55502-1 (2006)

${ }^{20}$ M. I. Panevsky and R. D. Bengston, Phys. Plasmas 11, 4196 (2004)

${ }^{21}$ R. H. Goulding, F. W. Baity, D. A. Rasmussen, D. O. Sparks, M. D. Carter and Y. Yoshitaka, Bull. Am. Phys. Soc. 52, 139 (2007)

${ }^{22}$ R. W. Boswell and F. F. Chen, IEEE Trans. Plasma Sci. 25, 1229 (1997)

${ }^{23}$ F. F. Chen and R. W. Boswell, IEEE Trans. Plasma Sci. 25, 1245 (1997) 
${ }^{24}$ E. E. Scime, A. M. Keesee and R. W. Boswell, Phys. Plasmas 15, 058301 (2008)

${ }^{25}$ P. Aigrain, Proc. Int. Conf. Semiconductor Physics, 224 (1960)

${ }^{26}$ L. R. Storey, Philos. Trans. R. Soc. London A Math. Phys. Sci. 246, 113 (1953)

${ }^{27}$ Y. Mouzouris and J. E. Scharer, Phys. Plasmas 5, 4253 (1998)

${ }^{28}$ F. F. Chen and D. D. Blackwell, Phys. Rev. Lett. 82, 2677 (1999)

${ }^{29}$ R. Kinder and M. J. Kushner, J. Vac. Sci. Technol. A 19, 76 (2001)

${ }^{30}$ G. G. Borg, J. Bright and I. V. Kamenski, Plasma Phys. Controlled Fusion 40, 987 (1998)

${ }^{31}$ A. W. Degeling and R. W. Boswell, Phys. Plasmas 4, 2748 (1997)

${ }^{32}$ S. Cho and J. G. Kwak, Phys. Plasmas 4, 4167 (1997)

${ }^{33}$ R. T. S. Chen and N. Hershkowitz, Phys. Rev. Lett. 80, 4677 (1998)

${ }^{34}$ A. W. Degeling, N. Mikhelson, R. W. Boswell and N. Sadeghi, Phys. Plasmas 5, 572 (1998)

${ }^{35}$ M. M. Balkey, R. F. Boivin, J. L. Kline and E. E. Scime, Plasma Sources Sci. Technol. 10, 284 (2001)

${ }^{36}$ F. F. Chen, Plasma. Phys. Controlled Fusion 33, 339 (1991)

${ }^{37}$ C. Charles, Plasma Sources Sci. Technol. 18, R1 (2007)

${ }^{38}$ R. Tanberg, Phys. Rev. 35, 1080 (1930)

${ }^{39}$ E. Kobel, Phys. Rev. 36, 1636 (1930)

${ }^{40}$ A. V. Gurevich, L. V. Pariiskaya and L. P. Pitaevskii, Sov. Phys. JETP. 22, 449 (1966)

${ }^{41}$ V. G. Eselevich and V. G. Fainshtein, Sov. Phys. JETP. 52, 441 (1980)

${ }^{42}$ G. Samir, K. H. Wright and N. H. Stone, Rev. Geophys. Space Phys. 21, 1631 (1982)

${ }^{43}$ G. Hairapetian and R. L. Stenzel, Phys. Rev. Lett. 61, 1607 (1988)

${ }^{44}$ G. Hairapetian and R. L. Stenzel, Phys. Rev. Lett. 65, 175 (1990)

${ }^{45}$ G. Hairapetian and R. L. Stenzel, Phys. Fluids B 3, 899 (1991)

${ }^{46}$ K. H. Wright, N. H. Stone and G. Samir, J. Plasma Phys. 33, 71 (1985)

${ }^{47}$ P. Wagli and T. P. Donaldson, Phys. Rev. Lett. 40, 875 (1978)

${ }^{48}$ N. Singh and R. W. Schunk, J. Geophys. Res. 88, 7867 (1983)

${ }^{49}$ S. A. Anderson, V. O. Jensen, P. Neilson and N. D’Angelo, Phys. Fluids 12, 557 (1969)

${ }^{50}$ P. Korn, T. C. Marshall and S. P. Schlesinger, Phys. Fluids 13, 517 (1970)

${ }^{51}$ H. Gunell, J. P. Verboncoeur, N. Bremming and S. Torvén, Phys. Rev. Lett. 77, 5059 (1996)

${ }^{52}$ T. Intrator, J. Menard and N. Hershkowitz, Phys. Fluids B 5, 806 (1992)

${ }^{53}$ P. Coakley and N. Hershkowitz, Phys. Fluids 22, 1179 (1979)

${ }^{54}$ P.A. Keiter, Experimental Investigation of Ion Temperature Anisotropy Driven Instabilities in a High Beta Plasma, Ph. D. Thesis, West Virginia University, Morgantown (1999) 
${ }^{55}$ M. M. Balkey, Optimization of a Helicon Plasma Source for Maximum Density with Minimal Ion Heating, Ph. D. Thesis, West Virginia University, Morgantown (2000)

${ }^{56}$ J. L. Kline, Slow Wave Ion Heating and Parametric Instabilities in the HELIX Helicon Source, $\mathrm{Ph}$. D. Thesis, West Virginia University, Morgantown (2002)

${ }^{57}$ X. Sun, A Study of Ion Acceleration, Asymmetric Optical Pumping and Low Frequency Waves in Two Expanding Helicon Plasmas, Ph. D. Thesis, West Virginia University, Morgantown (2005)

${ }^{58}$ A. M. Keesee, Neutral Density Profiles in Argon Helicon Plasmas, Ph. D. Thesis, West Virginia University, Morgantown (2005)

${ }^{59}$ R. A. Hardin, Measurement of Short-Wavelength Electrostatic Fluctuations in a Helicon Plasma Source, Ph. D. Thesis, West Virginia University, Morgantown (2008)

${ }^{60}$ I. A. Biloiu, Laser Induced Fluorescence Studies of Ion Acceleration in Single and Multiple Species Expanding Plasmas, Ph. D. Thesis, West Virginia University, Morgantown (2009)

${ }^{61}$ M. Light and F. F. Chen, Phys. Plasmas 2, 1084 (1995)

${ }^{62}$ F. F. Chen, UCLA Internal Report, PPG-1401 (1992)

${ }^{63}$ C. Charles, R. W. Boswell and R. K. Porteus, J. Vac. Sci. Technol. A 10, 398 (1992)

${ }^{64}$ C. Charles, J. Vac. Sci. Technol. A 11, 157 (1993)

${ }^{65}$ C. Charles and R. W. Boswell, J. Vac. Sci. Technol. A 13, 2067 (1995)

${ }^{66}$ F. F. Chen, X. Jiang and J. D. Evans, J. Vac. Sci. Technol. A 18, 2108 (2000)

${ }^{67}$ A. J. Perry and R. W. Boswell, Appl. Phys. Lett 84, 332 (2004) 


\section{CHAPTER 3: Standard Plasma Diagnostics}

It is convenient to divide plasma diagnostics techniques into three broad categories: ex situ, in situ intrusive and in situ non-intrusive. The common characteristic of ex situ measurement techniques is that they sample a portion of the plasma reactors contents and transfer it elsewhere for examination. On the other hand, the division of in situ techniques into intrusive and non-intrusive is more arbitrary. To some extent, any diagnostic technique perturbs the plasma. In some cases the perturbations may be so slight that they can be neglected, while in other cases they may be quite considerable and have included properly for correct analysis.

All the diagnostic techniques discussed in this chapter are in situ methods; both in situ intrusive and in situ non-intrusive. An example of an in situ diagnostic which is generally considered intrusive is a Langmuir probe. In this case, a physical object is inserted into the plasma, thereby perturbing it. However, depending upon the information desired, this perturbation may or may not be negligible. Another similar diagnostic is the electrostatic two tip probe. The Retarding Field Energy Analyzer (RFEA) is a relatively larger metallic object inserted in the plasma. Its perturbation is non- negligible and has to be considered during RFEA data analysis. One in situ non-intrusive method discussed in this chapter is laser induced fluorescence (LIF); as long as the injection of the laser and collection of the emitted fluorescence light is accomplished from outside the plasma chamber.

Although none of the diagnostic techniques discussed in this chapter are completely original in concept, most of the hardware has been modified to suite our experimental requirements. The development of a novel in situ non-intrusive plasma diagnostic, continuous wave cavity ring down spectroscopy (CW-CRDS), will be discussed in detail in Chapter 6.

\subsection{Langmuir Probe}

Electrostatic probes are undisputedly one of the oldest and most widely used diagnostic tools, particularly in low temperature plasmas. The technical description and the first theoretical explanation of the electrostatic probe were developed by Irving Langmuir, ${ }^{1}$ hence it is widely known as the Langmuir probe. In its simplest form, the 
Langmuir probe consists of a single conductor inserted into plasma to obtain local parameters such as the electron temperature and density. Measurements, in general, are performed by applying a bias to the conductor with a voltage and measuring the current drawn. Albeit a simple measurement to perform, the analysis of a Langmuir probe data is non-trivial. In particular, analysis of Langmuir probe measurements in drifting, nonMaxwellian or collisional plasmas require comparison with complex theoretical models. Thus a typical simplifying assumption in the analysis is that the plasma investigated is a stationery plasma with a Maxwellian velocity distribution. As with all probes physically introduced in the plasma, the perturbative effects of the probe on the plasma can be significant. Excellent reviews on both the theory and the operation of Langmuir probes are available in the literature, e.g., Chen, ${ }^{2}$ Schott, ${ }^{3}$ Hutchinson, ${ }^{4}$ Hershkowitz $^{5}$ and Demidov et al. ${ }^{6}$ Only the most relevant aspects of the probe theory, design and data analysis are presented here.

\subsubsection{Langmuir Probe Theory}

A classic Langmuir probe measurement consists of applying a bias voltage to a conductor immersed in plasma and measuring the current drawn. By sweeping the probe through a range of bias voltages and measuring the corresponding currents, the relationship commonly known as I-V characteristic or I-V trace is obtained. An ideal I-V trace is shown in Figure 3.1

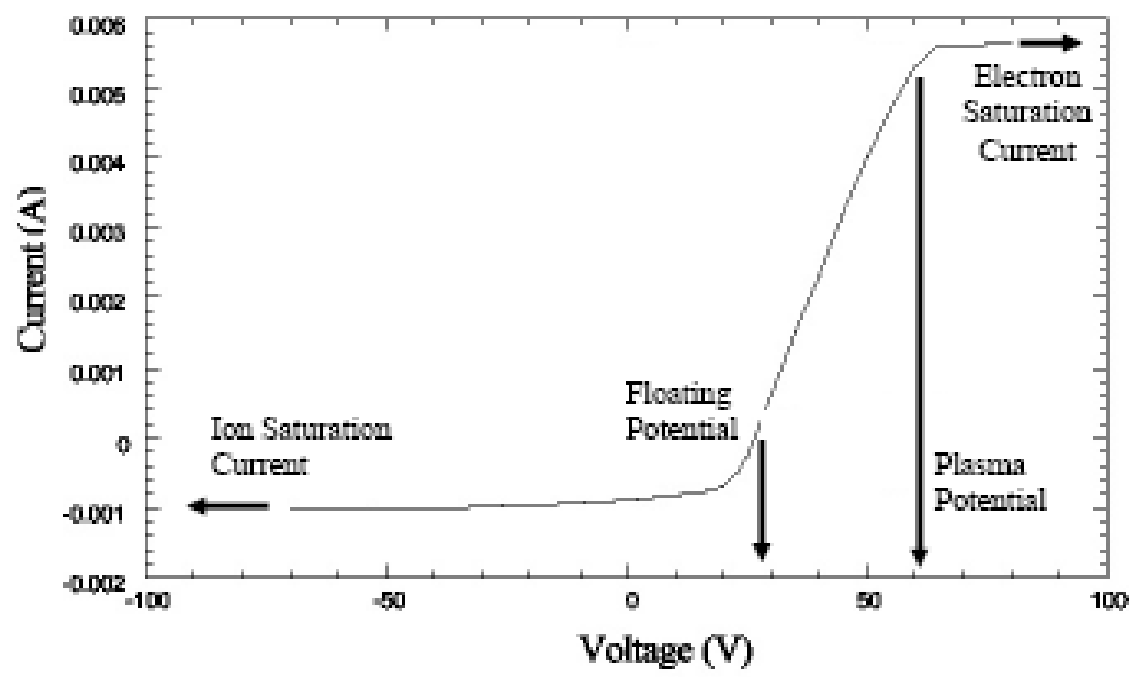

Figure 3.1. Idealized Langmuir probe I-V characteristic. Figure obtained from Ref.[7] 
If a Langmuir probe is placed in the plasma without a bias or connection to the electrical ground, the probe would charge negatively until reaching a potential at which the current to the probe vanished. The potential at which the current vanishes is called the "floating potential," i.e. the potential at which the electron and ion fluxes to the probe are equal. For a typical quasi-neutral plasma, the probe charges negatively because the electrons have a larger flux to the probe than the ions due to the lighter mass and a larger mean velocity of the electrons.

Variation of the probe's bias voltage is accomplished by connecting the probe to a high impedance voltage source. When the applied voltage is more negative than the floating potential, the probe attracts ions and repels the electrons, resulting in a net positive current. As the negative bias is increased and all nearby electrons are collected, the ion current will reach a maximum possible current in the ideal case. This maximum ion current is called the ion saturation current. A similar process occurs when the Langmuir probe is biased more positive than the floating potential. Large positive biases draw larger and larger electron currents until the electron saturation current is reached. The bias voltage at which the slope of the I-V trace begins to decrease near the electron saturation ( $2^{\text {nd }}$ knee) is the plasma potential. At this voltage there is no potential difference between the probe and the plasma. The magnitude of the electron saturation current is larger than the magnitude of the ion saturation current because the electrons are more mobile than the ions. Both the electron and ion saturation currents depend on the size of the probe, the plasma density and the electron temperature.

To determine the plasma parameters from the I-V characteristic, one assumes that the plasma constituents obey a Maxwellian velocity distribution, are non-drifting, and collisionless and that there is no ambient magnetic field. The total electric current, $I\left(V_{0^{-}}\right.$ $V_{p}$ ), collected by the probe, in the region just above the floating potential is approximated by $^{4}$

$$
I\left(V_{0}-V_{p}\right)=n_{e} e A_{p}\left(\frac{T_{e}}{m_{i}}\right)^{1 / 2}\left[\frac{1}{2}\left(\frac{2 m_{i}}{\pi m_{e}}\right)^{1 / 2} \exp \left(\frac{e\left(V_{0}-V_{p}\right)}{T_{e}}\right)-\frac{A_{s}}{A_{p}} \exp \left(-\frac{1}{2}\right)\right]
$$


where $n_{e}$ is the plasma density, $e$ is the electronic charge, $A_{p}$ is the surface area of the probe, $T_{e}$ is the electron temperature, $m_{e}$ and $m_{i}$ are electron and ion mass respectively, $V_{0}$ is the applied voltage, $V_{p}$ is the plasma potential and $A_{s}$ is the area of the sheath surface. The sheath around a Langmuir probe is a region of space a few Debye lengths thick. The sheath is a region of spatially varying potential and is created when the ions in the plasma shield the potential applied to the probe. ${ }^{8}$ The second term inside the brackets of the Equation 3.1 is the ion saturation current, $I_{s i}$, in unmagnetized plasma

$$
I_{s i}=-e J_{i}=-0.61 e n_{e} A_{p} \sqrt{T_{e} / m_{i}} .
$$

There are two unknowns in Equation 3.1, the plasma density and the electron temperature. The derivative of Equation 3.1 with respect to the voltage seen by the plasma $\left(V=V_{0}-V_{p}\right)$ is

$$
\frac{d I(V)}{d V} \cong \frac{e}{T_{e}}\left(I-I_{s i}\right)+\frac{d I_{s i}}{d V}
$$

Since $d I_{s i} / d V$ « $d I(V) / d V$, Equation 3.3 can be rewritten to provide an estimate of the electron temperature

$$
T_{e}=\frac{e\left[I(V)-I_{s i}\right]}{\frac{d I(V)}{d V}} .
$$

Experimentally, the electron temperature is calculated by performing a linear fit to $\ln \mid \mathrm{I}$ $I_{s i} \mid$ versus $V$ and the electron temperature is the inverse of the slope of the fit. Using the calculated electron temperature and the measured ion saturation current, the electron density is determined via Equation 3.2.

Cylindrically Langmuir probes cannot be driven into electron saturation in high density plasmas such as helicon source plasmas because the sheath surrounding the probe continues to expand and collects more electrons as the voltage is increased. The collected electron current then never reaches saturation. ${ }^{7}$ Since the Langmuir probe does not reach 
electron saturation, the plasma potential at the "knee" is not directly measured and has to be approximated.

For $T_{e}>T_{i}$, the floating potential is related to the plasma potential through a simple relationship. Given that the ion current at the floating potential is

$$
j_{i}=0.25 n e \sqrt{\frac{8 k_{B} T_{i}}{\pi m_{i}}} .
$$

while the electron current (which is equal to the ion current at this voltage) at the floating potential is

$$
j_{e}=0.25 n e \sqrt{\frac{8 k_{B} T_{e}}{\pi m_{e}}} \exp \left(\frac{e\left(V_{f}-V_{p}\right)}{k_{B} T_{e}}\right) \text {, }
$$

where $k_{B}$ is the Boltzmann's constant. Since the total probe current is zero at the floating potential we have (with some rearrangement of terms)

$$
V_{p}=V_{f}+\frac{k_{B} T_{e}}{2 e} \ln \left(\frac{T_{e} m_{i}}{T_{i} m_{e}}\right) .
$$

Under the constraint $T_{e}>T_{i}, T_{i}$ can be replaced by $T_{e}$ in Equation 3.7 and for argon ions ( $m_{i}=40 m_{p}$, where $m_{p}$ is the mass of a proton), Equation 3.7 becomes $^{9}$

$$
V_{p} \approx V_{f}+5.6 T_{e} .
$$

Thus, in argon, the difference between the plasma and the floating potentials is approximately six times the electron temperature. So the slope of $d I / d V$ can be well approximated by $d I / d V_{\text {applied }}$ for a reasonable estimate of the electron temperature.

One major aspect of Langmuir probe theory that has been ignored so far is the effect of a magnetic field. Since the ions and electrons gyrate around the magnetic field 
lines and cross field transport is limited, the flux of particles to the probe is restricted. The total effect depends on the ratio of the gyroradius of each species to the size of the probe. When this ratio is much less than one for a particle species, that species is impeded from reaching the probe and Equations 3.1 through 3.4 must be modified by including collisional terms and estimates of cross field transport. ${ }^{4}$ Since electrons have a much smaller gyroradius, the electron flux to the probe is preferentially reduced. In our case, since we do not bias the probe into electron saturation for density measurements, only the effects on the ions are relevant.

For a magnetic field of 1000 Gauss in HELIX and ion temperature of $0.3 \mathrm{eV}$, the ion gyroradius is approximately $3.5 \mathrm{~mm}$ which is of the same order of magnitude as the probe tip length of $2 \mathrm{~mm}$ but larger than the tip diameter of $0.5 \mathrm{~mm} .{ }^{10}$ Including magnetic field effects, Equation 3.2 becomes

$$
I_{s i}=-0.49 e n_{e} A_{p} \sqrt{T_{e} / m_{i}}
$$

Another important effect on Langmuir probe measurements arise from rf fields in the helicon source. The rf fields constantly accelerate and decelerate the electrons towards the probe when the probe is near the floating potential, resulting in an error in the floating potential measurement and an apparent broadening of the electron velocity distribution function. ${ }^{11}$ A method, developed by Sudit and Chen, ${ }^{12}$ is used to compensate for the rf fields in helicon plasmas. The addition of a floating electrode that is exposed to the plasma potential fluctuations and connected to the probe tip through a small capacitor, forces the probe tip to follow the potential oscillations, thereby reducing the sheath impedance. The Langmuir probe used in these experiments has such a floating electrode, but it is not directly exposed to the plasma. A set of rf chokes are also connected in between the probe tip and the voltage source, increasing the impedance of the current measurement circuit at the rf frequency. 


\subsubsection{Langmuir Probe Design}

A schematic drawing of the Langmuir probe used in these experiments is shown in Fig 3.2. The probe tip is a $0.5 \mathrm{~mm}$ diameter graphite rod (commercially available for mechanical pencils) inserted into a $0.6 \mathrm{~mm}$ inner diameter alumina shaft and attached by a set screw to a copper base. A $10 \mathrm{nF}$ capacitor is also connected to the copper base. This assembly is placed inside a boron nitride $(\mathrm{BN})$ cap such that the probe tip extends into the plasma through a hole in the $\mathrm{BN}$ cap, while the opposite leg of the capacitor remains within the BN head so that it is not directly exposed to the plasma. The threaded BN cap attaches to the stainless steel probe shaft. A chain of rf chokes is attached to the copper base. The rf chokes are 0.25 Watt, shielded, resonant, inductors from Lenox-Fugle International, Inc.; each designed to block a particular range of rf frequencies.

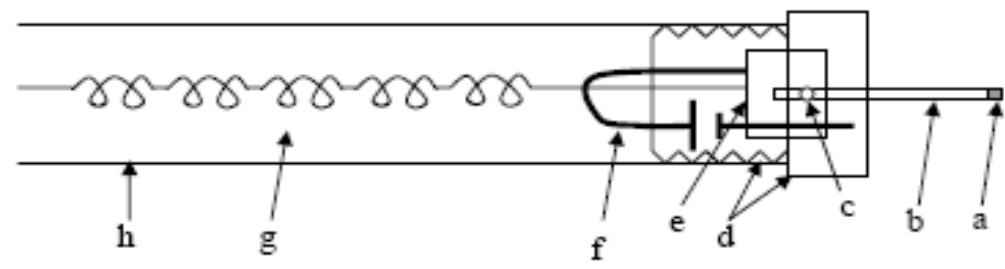

Figure 3.2. Schematic drawing of the Langmuir probe design including a) graphite probe tip, b) alumina shaft, c) set screw, d) threaded boron nitride cap, e) copper base, f) capacitor, g) chain of rf chokes and h) stainless steel probe shaft. Figure obtained from Ref. [13].

Starting from the copper base, the rf chokes are placed in the following order: 26, 53, 26, 13.2 and 6.8 MHz. The end of the rf chokes is then soldered to a shielded, coaxial probe wire that is attached to the BNC vacuum feedthrough at the far end of the probe shaft. Thermaflex tubing is used to cover the chain of rf chokes. Fig 3.3 is a picture of the Langmuir probe head used in HELIX. Note that the majority of the probe tip is less than $1 \mathrm{~mm}$ in diameter and thus plasma perturbation is minimized. 


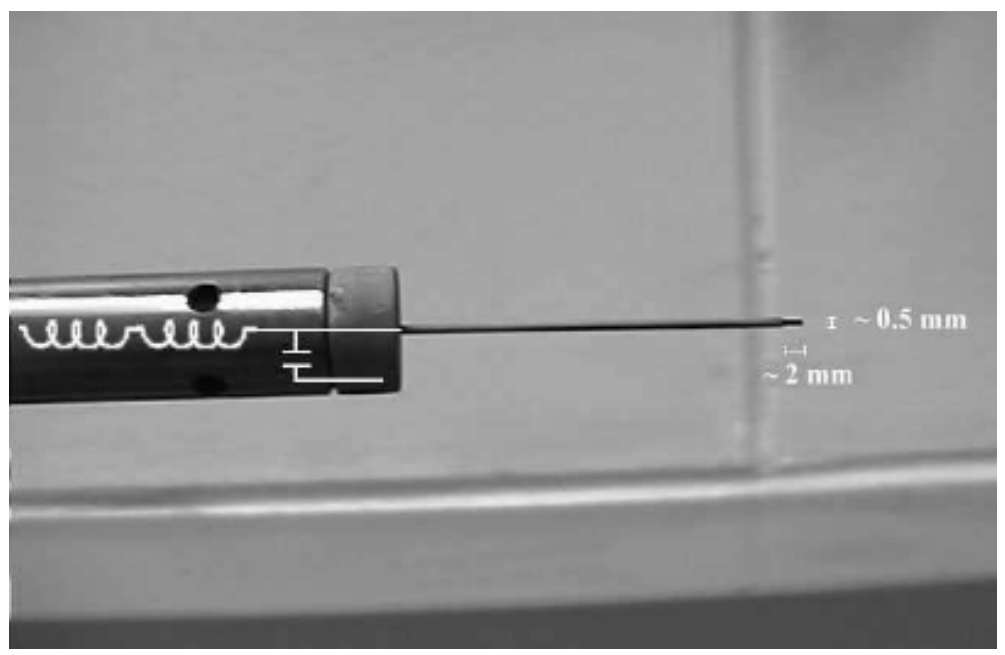

Figure 3.3. The Langmuir probe head. The exposed graphite tip is $2 \mathrm{~mm}$ long and runs the length of the alumina tube into the boron nitride cap. The capacitor and the inductor chains are shown schematically. Figure obtained from Ref. [14].

A high-impedance Keithley 2400 SourceMeter is used to measure the Langmuir probe I-V trace. The source meter applies the bias voltage to the probe, as well as measures the collected current. The source meter is controlled via a GPIB interface by custom software created in LabWindows ${ }^{\mathrm{TM}}$. A schematic drawing of the Langmuir probe measurement circuit is shown in Fig 3.4. The Langmuir probe measurements used in these experiments were obtained $50 \mathrm{~cm}$ downstream of the antenna at the position shown in Fig. 2.4.

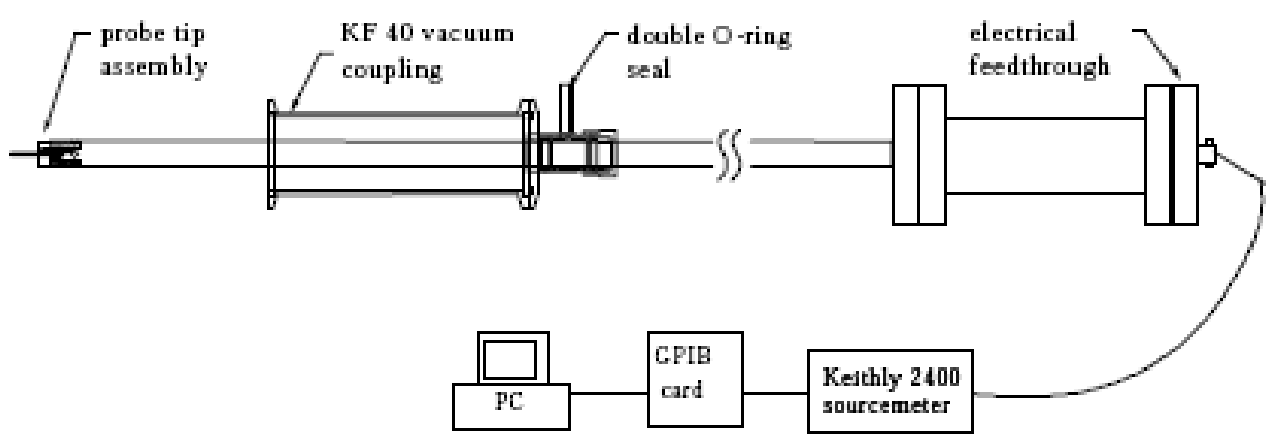

Figure 3.4. Schematic drawing of the Langmuir probe measurement circuit. Figure obtained from Ref. [14]. 


\subsection{Electrostatic Probe}

The electrostatic probe used for electrostatic fluctuation measurements is essentially a two-tipped Langmuir probe. The two tips are separated spatially to measure differences in floating potential. The electrostatic probe has no rf compensation because the objective was to measure fluctuations in the floating potential at frequencies up to the rf driving frequencies. We assume that the fluctuations are that of the floating potential and not due to fluctuations in the electron temperature. The emissive probes ${ }^{15}$ typically used to determine electron temperature fluctuations do not work well in steady state helicon plasma with densities of the order of $10^{13} \mathrm{~cm}^{-3}$.

\subsubsection{Electrostatic Probe Theory}

Since the conducting tips of the electrostatic probe are unbiased, they charge up to the voltage at which no current flows to the probes, i.e. the floating potential. When additional charged particles are driven to the probe, by changes in the nearby plasma potential arising from electrostatic waves, the particle fluxes to the probe tips change. The changes in the particle flux cause changes in the floating potential. By measuring the floating potential fluctuations at two spatially separated locations, the local electric field can be determined from $\boldsymbol{E}=\Delta \varphi / d$, where $\Delta \varphi=\varphi_{1}\left(x_{1}\right)-\varphi_{2}\left(x_{2}\right)$ is the difference in floating potential at the location of the two probe tips and $d=x_{1}-x_{2}$ is the spatial separation of the two probe tips. The electrostatic probe can also be used to measure the wavelength of traveling electrostatic waves by determining the phase difference between the two probe tips for a known separation distance. For a probe tip separation “ $d$ ”, and a measured phase difference of $\Delta \theta$ between the two probe tips, the wave number of a travelling wave is given by $k=\Delta \theta / d$. From the individually recorded time series of the fluctuating potentials measured by each probe, a power spectrum can be calculated which gives a measure of the strength of the fluctuations at the frequencies of interest. Knowing the frequencies and the wavelengths, the dispersion relation ( $\omega$ versus $k$ ) for the wave is determined. 


\subsubsection{Electrostatic Probe Design}

The electrostatic probe used in this work ${ }^{16}$ consists of two tungsten tips, $2 \mathrm{~mm}$ in length and $0.33 \mathrm{~mm}$ in diameter (Fig. 3.5). The center-to-center distance between the tips is $0.61^{+0.33}-0.13 \mathrm{~mm}$. The upper limit includes the center to center separation distance added to the individual radius of each tip, while the lower limit is based on the resolution limit of the method used to measure the tip spacing. The tungsten tips protrude from a 6bore alumina shaft that is $2 \mathrm{~mm}$ in diameter and extend $7.6 \mathrm{~cm}$ from a boron nitride (BN) cap. Just inside the base of the BN cap, the tungsten wires are soldered to the wires that bring the signals out to the vacuum BNC feedthrough. The thermoflex covered the signal wires and kept them from touching inside the probe shaft. The signal from each tip is connected to the Joerger VTR10012, a 12-bit resolution digitizer, with RG223U double shielded cables. The data records are transferred from the digitizer via a MIXI bus to a PC for later analysis. The various possible orientations of the probe are shown in Fig. 3.6. For a maximum measured phase difference of $\pi$ and a tip separation of $0.061 \mathrm{~cm}$, the maximum measurable wave number is $\pm 51.5 \mathrm{rad} / \mathrm{cm}$.

a)

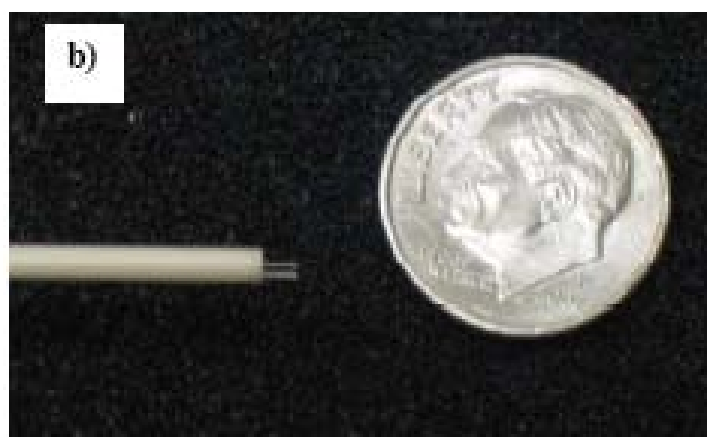

Figure 3.5. (a) End view of the electrostatic double probe. The dark portions represent the locations of the tungsten tips in the 6-bore alumina. (b) The electrostatic double probe. Figure obtained from Ref. [16]. 


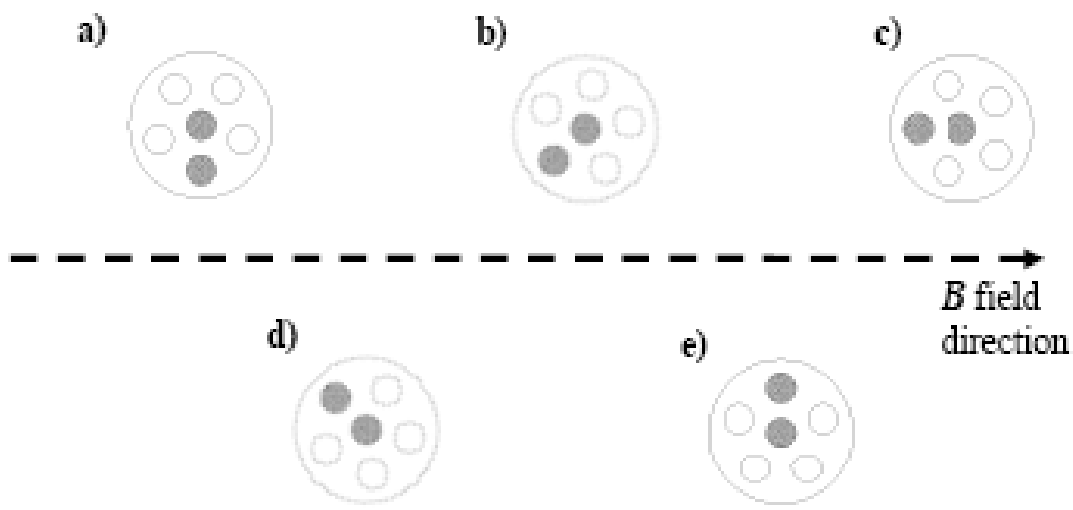

Figure 3.6. Possible electrostatic double probe orientations with respect to the direction of the magnetic field: (a) perpendicular, (b) + 45 degrees, (c) parallel, (d) - 45 degrees and (e) antiperpendicular. Figure obtained from Ref. [16].

\subsubsection{Electrostatic Probe Analysis}

To estimate the wave number of a wave, at a specific instant in time, using two spatially separated probes, the phase difference $(\theta)$ between the signals measured by the two probes has to be measured. For a single traveling wave, having typical ( $\omega t-\boldsymbol{k} \boldsymbol{r})$ behavior and traveling past the two tips, a phase difference $(\theta=\boldsymbol{k} \boldsymbol{\bullet r})$ occurs because of the finite transit time of the wave. For a measured phase difference and a known probe separation, $r$, the wave number, $k$, is determined from the ratio $\theta / r$.

For ideal sinusoidal signals, the phase difference can be directly measured from the time series measurements of the two signals. However, typical signals are distorted by harmonics of the wave and noise. One way to find the phase differences at specific harmonics is to calculate the cross-power spectrum of the two measured time series. The cross-power spectrum is the product of the Fast Fourier Transform (FFT) of one time series and the complex conjugate of the FFT of the other time series. Mathematically, let $f_{1}\left(x_{1}, t\right)$ and $f_{2}\left(x_{2}, t\right)$ represent the two individual time series. The FFTs are defined as

$$
\Phi_{1}\left(x_{1}, \omega\right)=\int_{-\infty}^{\infty} f_{1}\left(x_{1}, t\right) \cos (\omega t) d t-i \int_{-\infty}^{\infty} f_{1}\left(x_{1}, t\right) \sin (\omega t) d t
$$

and 


$$
\Phi_{2}\left(x_{2}, \omega\right)=\int_{-\infty}^{\infty} f_{2}\left(x_{2}, t\right) \cos (\omega t) d t-i \int_{-\infty}^{\infty} f_{2}\left(x_{2}, t\right) \sin (\omega t) d t
$$

where $x_{1}$ and $x_{2}$ are the respective probe tip locations. The cross-power spectrum is given by the product of Eqn. 3.10 and the complex conjugate of Eqn. 3.11;

$$
P_{12}(\Delta x, \omega)=\Phi_{1}\left(x_{1}, \omega\right) \Phi_{2}^{*}\left(x_{2}, \omega\right) \text {, }
$$

where $\Delta x=x_{1}-x_{2}$, is the spatial separation of the probe tips. Expanding Eq. 3.12, by substitution of Eqns. 3.10 and 3.11, we get the cross-power spectrum as a function of the real and imaginary parts of each FFT;

$$
P_{12}(\Delta x, \omega)=\left(\varphi_{1 \mathrm{Re}} \varphi_{2 \mathrm{Re}}+\varphi_{1 \mathrm{Im}} \varphi_{2 \mathrm{Im}}\right)+i\left(\varphi_{1 \mathrm{Im}} \varphi_{2 \mathrm{Re}}-\varphi_{1 \mathrm{Re}} \varphi_{2 \mathrm{Im}}\right)
$$

In the complex plane, the phase difference of the cross-power spectrum and hence of the two time series, is simply the angle between the real and the imaginary vectors:

$$
\Theta(\omega)=\tan ^{-1}\left(\frac{\left(\varphi_{1 \mathrm{Im}} \varphi_{2 \mathrm{Re}}-\varphi_{1 \mathrm{Re}} \varphi_{2 \mathrm{Im}}\right)}{\left(\varphi_{1 \mathrm{Re}} \varphi_{2 \mathrm{Re}}+\varphi_{1 \mathrm{Im}} \varphi_{2 \mathrm{Im}}\right)}\right)
$$

An implicit assumption in these calculations is that the signal-to-noise ratio is very large. When the signals are relatively low in amplitude with respect to the noise, ensemble averaging of many cross-power spectra can significantly improve the precision of the phase measurements: the random errors decrease as $1 / \sqrt{M}$, where $M$ is the number of data samples recorded. ${ }^{17}$

Two difficulties associated with using the cross-power spectrum technique with pairs of spatially separated probes are the large amounts of data required for each measurement and aliasing in both the frequency and spatial domains. In power spectrum measurements at a single spatial location, aliasing occurs when a periodic signal's frequency is larger than the Nyquist frequency $\left(f_{\text {Nyquist }}=0.5 \times\right.$ sample rate) of the data 
acquisition system. In an FFT of an aliased time series measurement, the periodic signal spectral power will appear downshifted in frequency. Additional spectral peaks at harmonics of the artificially downshifted frequency are also likely to appear in the FFT. For the sampling rate of $100 \mathrm{MHz}$, the Nyquist frequency for the experiments is $50 \mathrm{MHz}$. So for the low frequency measurements in the experiments $(17.5 \mathrm{kHz})$, temporal aliasing is ignorable.

For wave number measurements using a pair of spatially fixed probes, spatial aliasing occurs when the wavelength of the wave is smaller than the separation distance between the two tips (Fig. 3.7). For the situation depicted in the figure, the measured phase differences are the same even though their wavelengths are not. As seen in Fig. 3.7, the probe separation places a lower bound on the wavelength $(\lambda)$ of the waves that can be resolved: $\lambda_{\min }=2 d$ where $d$ is the probe separation distance. With a probe tip separation of $0.061 \mathrm{~cm}$, wavelengths of the order of $0.12 \mathrm{~cm}(\mathrm{kmax} \sim 51.5 \mathrm{rad} / \mathrm{cm})$ are measurable. Hence for our experimentally measured wavelengths of $30 \mathrm{~cm}$, spatial aliasing is not significant.
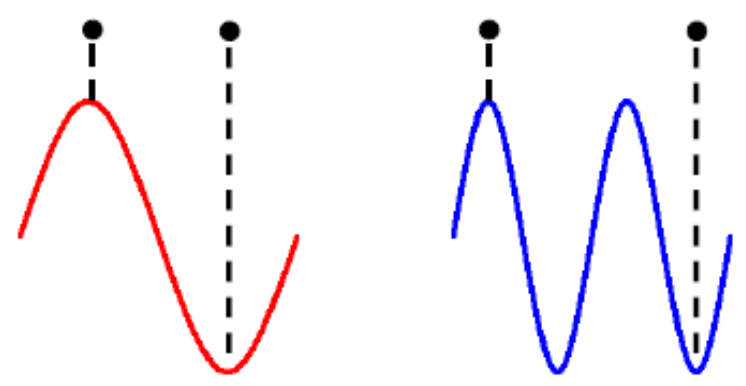

Figure 3.7. An illustration of spatial aliasing for a pair of fixed probes. The black dots and dashed lines represent the spatial location of the probe tips while the color sinusoids represent the wave amplitudes at an instant in time. Figure obtained from Ref. [16].

\subsection{Retarding Field Energy Analyzer (RFEA)}

Retarding Field Energy Analyzers (RFEA) have been used extensively over the last couple of decades to measure the ion energy distribution (IED) in a variety of plasma reactors and for a variety of discharge operating conditions. ${ }^{18,19,20,21,22,23,24,25,26,27,28}$ In its most simple form, the potential of a grid placed in front of a beam of ions is increased 
until the beam flux is stopped. By collecting and measuring the current due to the ions passing through the grid, as a function of the grid potential, the integrated IED is obtained. The true IED is obtained by differentiating the current-potential characteristic.

Most of the early RFEA designs required differential pumping to ensure that the analyzer dimensions are smaller than the ion mean free path in the device. ${ }^{18,19}$ The need for differential pumping made the designs for these RFEA very cumbersome and prohibited more widespread use. Ingram and Braithwaite ${ }^{20}$ designed and built a compact RFEA that did not require differential pumping in the pressure ranges being investigated. The analyzer design, which incorporated two grids and a Faraday cup detector, was very compact and had a total depth of only $0.75 \mathrm{~mm}$, less than the mean free path of the ions in the pressure range being studied. The first grid behind the entrance orifice was biased negatively to prevent electrons from entering the analyzer and a potential sweep was applied to the ion current collector to discriminate ions with different energies. This simple and compact design was utilized by several research groups $\mathrm{s}^{21,22,23,24,25}$ in a variety of plasma reactors for various plasma chemistries. The two grid design, though, can lead to problems with secondary emission within the analyzer. Later efforts to increase the

efficiency and resolution of the RFEA involved the use of one or more extra grids, ${ }^{26,27,28}$ to minimize secondary electron emission from the metallic surfaces within the analyzer.

\subsubsection{Theory of RFEA}

The working principles of an RFEA can be easily understood from the schematic diagram of a typical multi-grid RFEA shown in Figure 3.8. The RFEA begins with a small entrance slit in the probe surface that admits the plasma. The probe entrance is aligned so that the local magnetic field is normal to the plane of the aperture. Thus, the measurements are of the energy distribution of the parallel component of the ion flux. The entrance slit (or in some designs, a grid just behind the entrance slit) is biased to a sufficiently large and constant negative potential $\left(\Phi_{e}\right)$ to repel most of the electrons entering from the plasma. A discriminator potential $\left(\Phi_{D}\right)$, applied to the next grid that the ions face, ranges from zero to a large positive value to repel ions. Only ions with a kinetic energy larger than $e \Phi_{D}$ cross this retarding grid. The next grid is biased to a constant negative potential $\left(\Phi_{S}\right)$ to suppress any secondary electrons produced by the ion 
bombardment on the collector and to repel any secondary electrons created by ion impact on the second grid. The current collected by the collector decreases as the potential on the discriminator increases, as more and more ions are repelled.

There are existing theories and modeling results ${ }^{25,29,30}$ on how the collector current measured as a function of the discriminator potential depends on the energy of the ions and relates to the ion velocity distribution function. It is generally believed that the first derivative $\left(\partial I_{C}\left(\Phi_{D}\right) / \partial \Phi_{D}\right)$ of the collector current $\left(I_{C}\right)$ with respect to the varying potential $\left(\Phi_{D}\right)$ applied to the retarding grid gives the ion velocity distribution $(f(v))$.

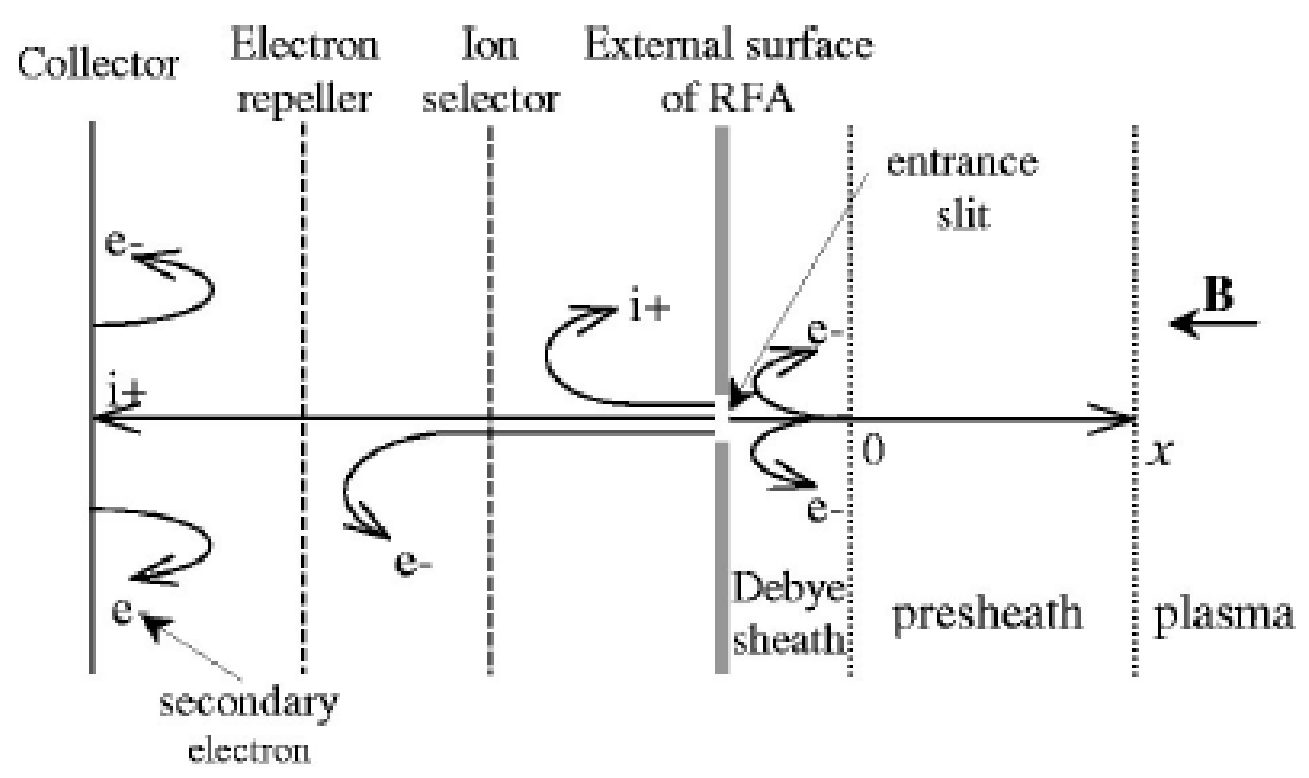

Figure 3.8. Schematic representation of a typical RFEA showing the sequence of grids and their effects on the charged particles. Figure obtained from Ref. [30].

To understand how a RFEA measurement actually relates to the ion velocity distribution we consider the simplest case of a perfectly anisotropic ion velocity distribution, with respect to the $\mathrm{z}$ axis (parallel to the magnetic field). Assume the $v_{x}$ and $v_{y}$ components of the ion velocity distribution function $(f(v))$ are ignorable by choosing them to be Dirac delta functions. Thus, we have a one dimensional ion velocity distribution function $f\left(v_{z}\right)$, which for the sake of simplicity, we shall call $f(v)$. The total ion density $(n)$ is then given by 


$$
n=\int_{0}^{+\infty} d n=\int_{-\infty}^{+\infty} f(v) d v
$$

If $g(E)$ denotes the ion energy distribution, then by definition, we have

$$
n=\int_{o}^{+\infty} d n=\int_{-\infty}^{+\infty} g(E) d E
$$

Thus,

$$
\int_{-\infty}^{+\infty} f(E) d E=n=\int_{-\infty}^{+\infty} f(v) d v
$$

Since only the kinetic energies of ions with mass $m$ are considered;

$$
d E=d\left(\frac{1}{2} m v^{2}\right)=m v d v .
$$

Hence the velocity and the energy distribution functions of the ions entering the RFEA are related, up to a constant of integration, through

$$
f(v)=\operatorname{mvg}(E) .
$$

The total ion current density in the entrance plane of the analyzer $\left(I_{\text {ion }}\right)$ in this special one dimensional case is ${ }^{31}$

$$
I_{\text {ion }}=e \int v f(v) d v=\frac{e}{m} \int f(v) d E .
$$


In the RFEA, ions with energies larger than the potential applied to the discriminator grid pass through, so denoting the integration variable of the ion energy as $\xi$, Eqn. 3.20 becomes

$$
I_{\text {ion }}(E)=\frac{e}{m} \int_{0}^{E} f\left[\left(\frac{2 \xi}{m}\right)^{1 / 2}\right] d \xi \text {. }
$$

In the ideal case, all the ions reaching the entrance plane of the RFEA pass unhindered through the grids when the discriminator potential $\left(\Phi_{D}\right)$ is zero i.e. grounded and contribute to the current $\left(I_{C}\right)$ collected by the collector. As $\Phi_{D}$ is swept from zero to more positive values, the measured $I_{C}$ is decreases since the slower ions are repelled. In terms of the cutoff energy, the current collected by the collector is

$$
I_{C}(E)=\frac{e}{m} \int_{E}^{\infty} f\left[\left(\frac{2 \xi}{m}\right)^{1 / 2}\right] d \xi .
$$

The difference between the number of ions reaching the entrance grid of the RFEA from the plasma and the collector grid, at a given energy $E$, is due to the potential applied to the discriminator grid. Thus, $E$ (measured in $\mathrm{eV}$ ) can be replaced by the product of the elementary charge $e$ and the discriminator grid potential $\left(\Phi_{D}\right)$ (measured in Volts). Hence, the ion velocity distribution function is measured by calculated the derivative of the collected current with respect to the applied retarding potential

$$
f(v)=\frac{m}{e}\left[\frac{d I_{C}(E)}{d E}\right]=\frac{m}{e^{2}}\left[\frac{d I_{C}\left[\Phi_{D}\right]}{d \Phi_{D}}\right] .
$$

If the transparency of the grid are not $100 \%, f(v)$ is reduced by some factor. So, generally the ion velocity distribution function (IVDF), $f(v)$, is understood to be the first derivative of the collector current with respect to the discriminator voltage up to some arbitrary constant that depends on the design of the RFEA probe. 
It is worth noting, since it has been a point of discussion between research groups, that it is customary to plot the calculated IVDF versus $E$ for convenience. Such plots are a concern for purists since it is rather odd to plot $f(v)$, which is clearly defined as a function of $v$, versus $E$. It is possible to rescale the abscissa using $v=(2 E / m)^{1 / 2}$ and compress $f(v)$ along the abscissa; converting the plot of $f(v)$ into a real ion velocity distribution function. However, according to the measurement principles of the RFEA, since the ion energies are measured in terms of energy units (in $\mathrm{eV}$ ) and they are not an actual velocity measurement (in units of $\mathrm{m} / \mathrm{s}$ ), scientists generally accept the incongruity of plotting $f(v)$ as a function of $E$.

\subsubsection{Design and Schematics}

For probe measurements of the IVDF, a compact RFEA is inserted to the axis of the experiment, downstream of the source (position F in Fig. 2.3), and aligned so that the RFEA aperture faces the upstream (helicon source) direction. The RFEA used is WVU is based on the design schematics provided by the Australia National University (ANU) group and outlined in Ref. [29]. The RFEA consists of four closely spaced mesh screens placed behind a grounded aperture and held in grounded probe assembly (see Fig. 3.9). Inside the RFEA there are four "stacks", each consisting of a mica insulator and one 250 lines per inch nickel mesh glued to a copper support ring. The grids are kept in place by a mica insulating pocket. 30 AWG wires are soldered to the tabs in each of the support copper rings and attached to BNC connectors on the probe stem to be connected to individual power supplies. 

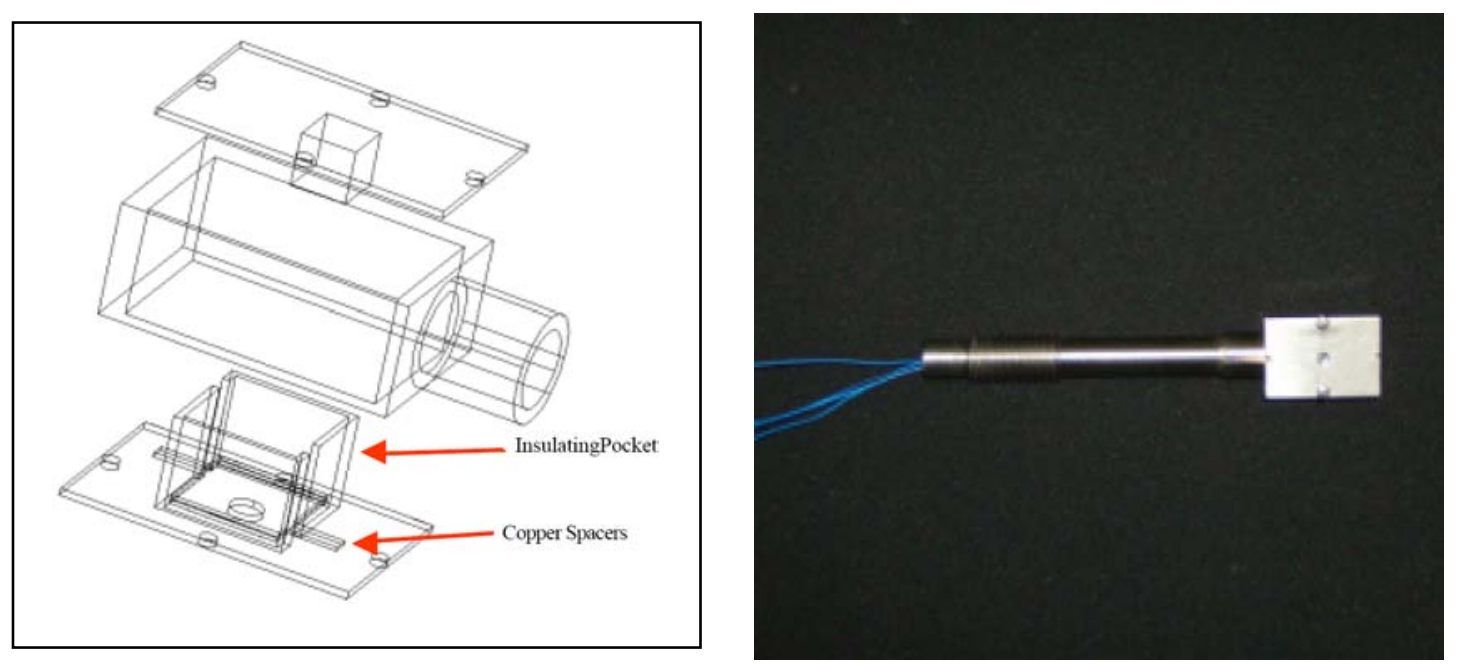

Figure 3.9. The RFEA housing: schematics and a photograph of the assemble probe.

The relative potentials applied to each grid are shown schematically in Fig. 3.10. The first grid, the electron repeller, is biased from -40 to $-70 \mathrm{~V}$ depending on the local plasma conditions. Since the typical electron temperature is $5 \mathrm{eV}$, the repeller bias is sufficient to exclude all but the most energetic electrons in the plasma. The second grid, the discriminator or retarder, is typically swept from $-5 \mathrm{~V}$ to $75 \mathrm{~V}$ with a linear ramp. Ions with energies larger than the discriminator potential continue on to the third grid. Because the grids are so closely spaced, discriminator sweep rates larger than $10 \mathrm{mHz}$ resulted in unacceptable levels of capacitive coupling to the third grid, the electron suppressor grid.

Since an "anomalous" ion signal arises if secondary electrons escape the collector, due to ion bombardment on the collector, the "suppressor grid" Is biased to -18 Volts with two 9 Volts batteries. The fourth grid, the collector, is a solid copper plate biased to -9 Volts. The current collected by the suppressor grid (due to those secondary electrons emitted from the collector with enough energy to overcome the $-9 \mathrm{~V}$ potential barrier) is added to the collector current with a fast amplifier with variable gain of 2 to 1001 .

The amplifier used with the RFEA is a switched gain DC amplifier with gains of 2, 11, 101 and 1001 and has a bandwidth of about $10 \mathrm{kHz}$. The amplifier is powered from a single 24 Volt DC floating plug-pack power supply with a ground reference established internally at 12 Volt positive from the negative supply; providing \pm 12 Volts to the amplifier. The output of the amplifier is buffered to drive a $50 \mathrm{ohm}$ load. The RFEA 
collector current develops a voltage across a 10k resistor, which is then amplified. The addition of the current collected by the suppressor grid and the collector current is performed before amplification. The electronics schematics were provided by ANU and the circuits built by researchers at WVU.

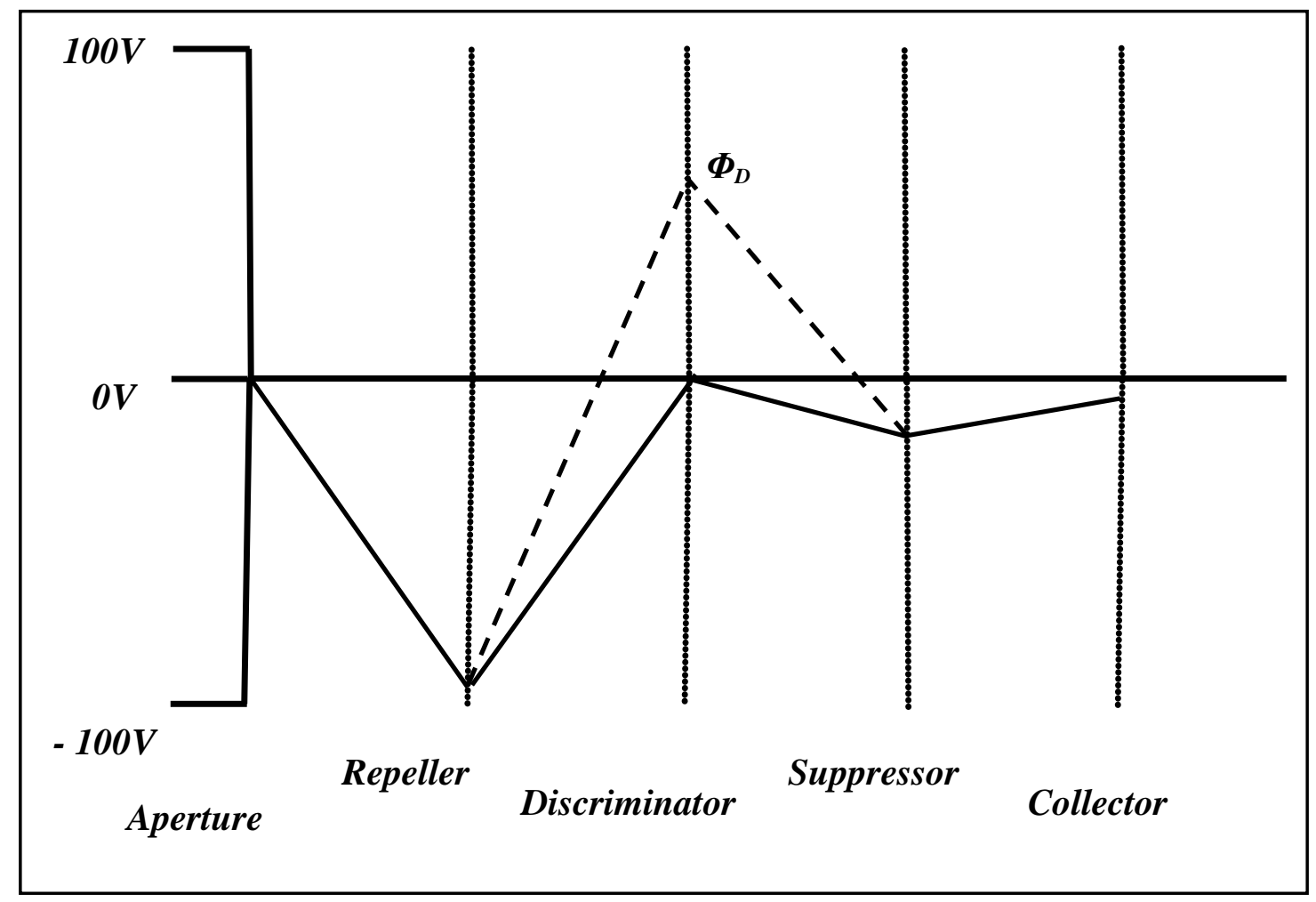

Figure 3.10. The RFEA bias voltages: the aperture is on the left and the vertical dotted lines represent the four grids. The dark line shows typical voltages applied to each grid. The dashed line shows the typical range of voltages applied to the discriminator grid.

\subsubsection{Data Analysis Methodology}

In our experiments (and for nearly all published RFEA measurements in plasmas), the support structure of the RFEA probe and the first grid are at electrical ground. Ions in the plasma are accelerated by the positive plasma potential towards the grounded first grid. Even if the average velocity of ions in the plasma is zero, the ion population entering the RFEA will do so with a bulk energy of $e \Phi_{P}$, where $\Phi_{P}$ is the plasma potential. Thus, the assumption that the particle distribution measured with a RFEA is non-drifting 
Maxwellian is incompatible with nearly every RFEA measurement ever performed. IVDF The current collected by an RFEA as a function of discriminator potential, $\Phi_{D}$ for a single drifting Maxwellian ion distribution (an ion beam) is given by

$$
I_{C}\left(\Phi_{D}\right)=\sqrt{\frac{n_{b}^{2} e^{2}}{2 m \pi}}\left[\sqrt{T_{b}} e^{-\left(\left(\sqrt{e \omega_{D}}-\sqrt{E_{b}}\right)^{2} / T_{b}\right)}+\sqrt{E_{b} \pi} \operatorname{erfc}\left[\left(\sqrt{e \Phi_{D}}-\sqrt{E_{b}}\right) / \sqrt{T_{b}}\right]\right],
$$

where $n_{b}$ is the beam density, $T_{b}$ the beam temperature, and $E_{b}$ the beam energy, i.e., the energy gained by the background ion population as it is accelerated to the RFEA by the local plasma potential. ${ }^{32}$ The derivative of Eq. (3.24) with respect to the discriminator potential is

$$
\left.d I_{C}\left(\Phi_{D}\right) / d \Phi_{D}=-n_{b} e \sqrt{1 / 2 m \pi T_{b}} e^{-\left(\left(\sqrt{e_{D}}-\sqrt{E_{b}}\right)^{2} / T_{b}\right.}\right)
$$

and thus the derivative is no longer a direct measure of the velocity distribution function. In the limit of zero beam energy, the right-hand side of Eq. (3.25) reduces to the original Maxwellian distribution. For finite beam energies, the width in energy space predicted by Eq. (3.25), i.e., the $1 /$ e folding points, is $\Delta \Phi_{D}=4 \sqrt{T_{b} E_{b}}$. In other words, the width of the peak resulting from a derivative of the collected current of an RFEA is not necessarily a measure of the ion temperature but is in fact proportional to square root of the product of the beam energy and the ion temperature.

Shown in Fig. 3.11 is the RFEA collector current versus discriminator voltage obtained in the LEIA expansion chamber near the position "F" in Fig. 2.3 for a repeller voltage of $-45 \mathrm{~V}$. The first "knee" in the raw RFEA collector current versus discriminator potential occurs at the local plasma potential. In a non-drifting plasma, the collector current would immediately begin to decrease for positive discriminator voltages. Thus, the raw RFEA data provides a direct measure of the local plasma potential; $25 \mathrm{eV}$ in this case. There is another "knee" at roughly $35 \mathrm{eV}$, indicative of an additional ion beam population in the ion distribution. 
The simplest means of estimating the energies of the two ion populations is to differentiate collected current with respect to the discriminator voltage, (see Fig. 3.12). The derivative analysis indicates that the local plasma potential is approximately 27 Volts (corresponding to the energy of the first peak) and that there is an ion beam with an energy of roughly $12 \mathrm{eV}$ relative to the background ion population (assuming both populations have been accelerated through the sheath in front of the probe). Based on the relative amplitudes of the two peaks, the derivative analysis also indicates a beam density roughly 50\% larger than the background ion density.

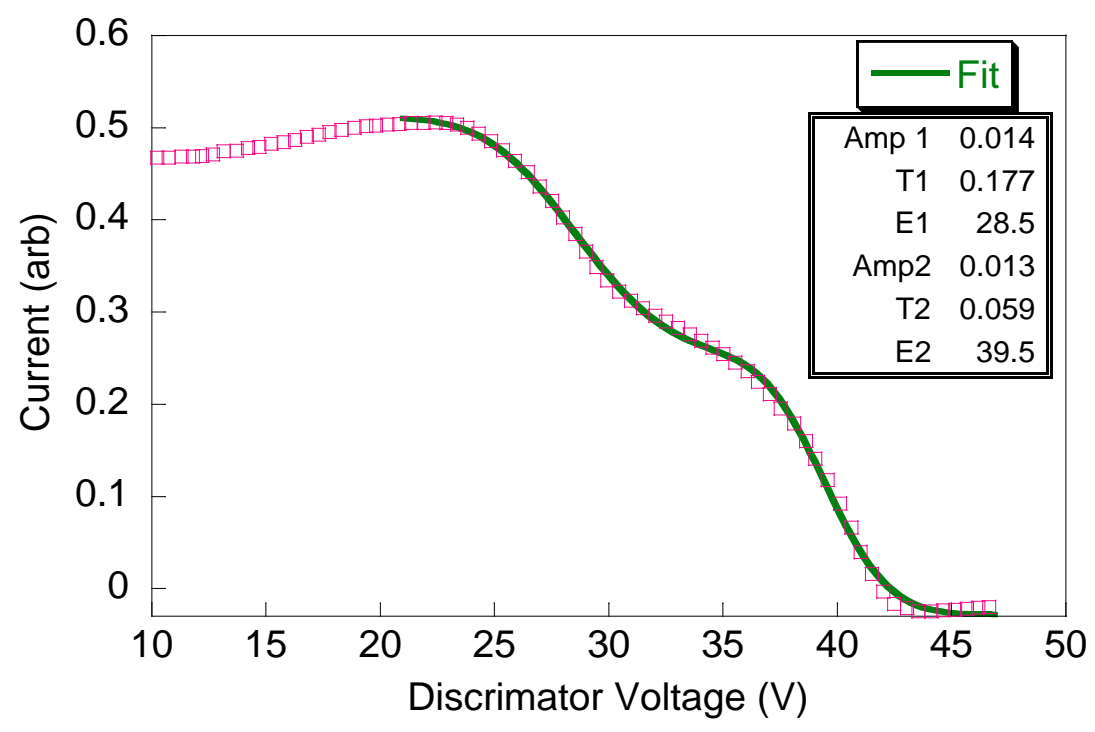

Figure 3.11. An example of RFEA data: collector current versus discriminator voltage. The red blank squares are the actual data. Signatures of ion beam populations are seen at about $25 \mathrm{~V}$ and about $35 \mathrm{~V}$. The solid line is a best fit to the RFEA data, using two drifting Maxwellian distributions, each described by Eqn. 3.25. (The best fit parameters are shown in the inset).

A slightly more sophisticated analysis method is to assume that the data of Figure 3.11 arise from a combination of two drifting ion populations (a background ion population accelerated by the plasma potential to ground potential difference and an ion beam population) as described by Eq. (3.24). Fitting the data with the sum of two beam populations yields the parent ion population shown by the green solid line in Fig. 3.13. In 
this case, the resultant beam density is equal to the background ion density and the energy of the ion beam remains about $12 \mathrm{eV}$.

As will be discussed in the next Chapter, this analysis method can be improved further by self-consisting including the effect of the acceleration of the background and beam ion populations into the RFEA.

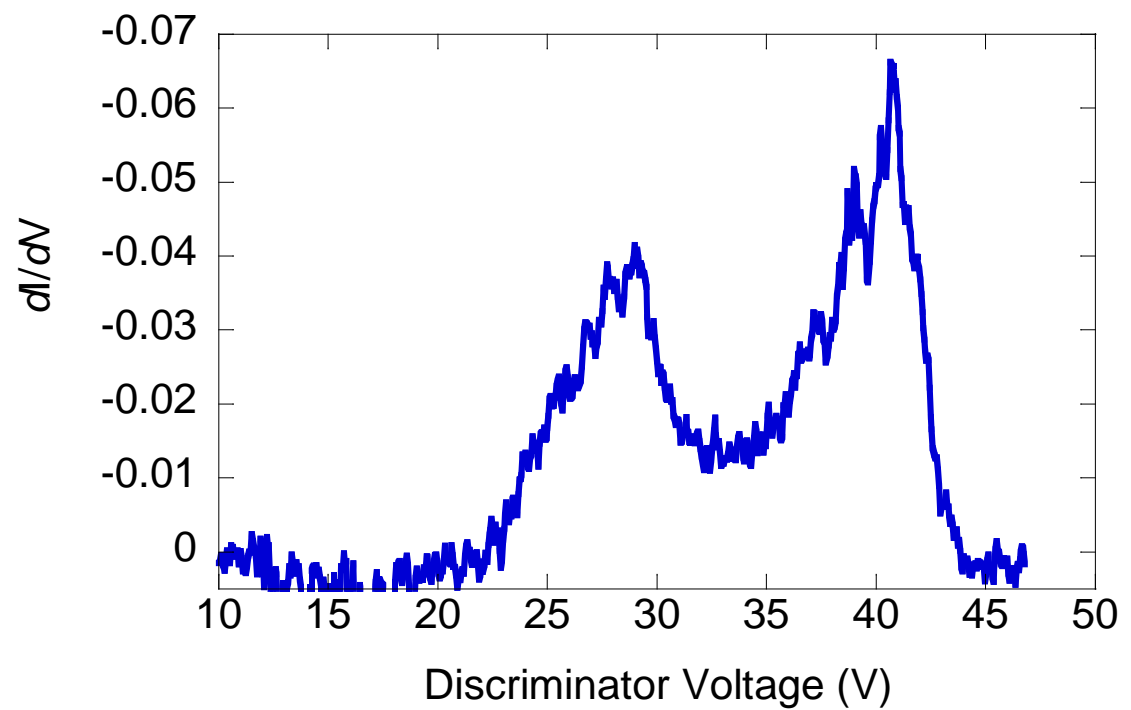

Figure 3.12. The derivative of the collector current with respect to the discriminator voltage, corresponding to the raw RFEA data shown in Figure 3.11.

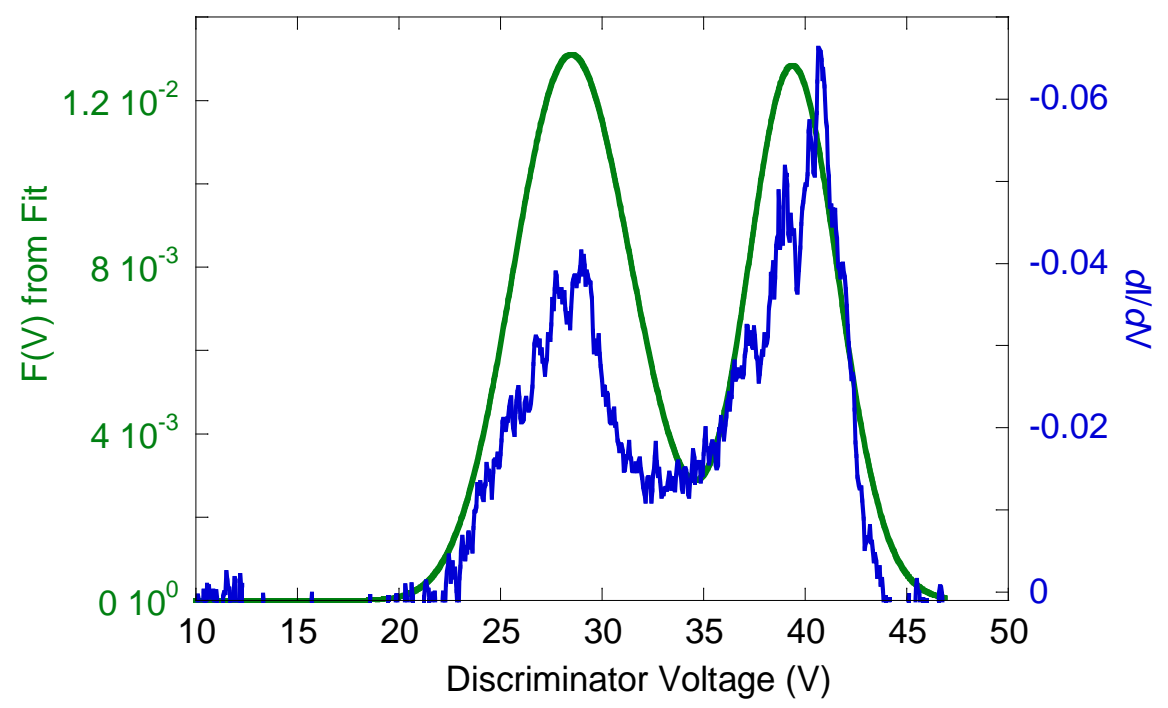

Figure 3.13. Comparing the two methods of finding IVDF from RFEA data. The green line is the parent ion population from the best fit of two drifting Maxwellian distributions. The blue line is the derivative of the collector current with respect to the discriminator voltage. 


\subsection{Laser Induced Fluorescence (LIF)}

Since the advent of the LASER (Light Amplification by Stimulated Emission of Radiation) in the 1960s, many new, laser-based, spectroscopic measurement techniques have been developed. Laser induced fluorescence (LIF) uses a laser tuned to a natural absorption line of an atom or ion to induce emission from the upper pumped state to either the same initial state or a different third state. The first laser induced fluorescence from molecules other than the lasing medium of a laser, was observed by Yardley and Moore in $1966 .{ }^{33}$ The first experiment to demonstrate LIF in plasmas was performed by Stern and Johnson ${ }^{34}$ using a single frequency argon ion laser in 1975. A tunable diode laser was first used by Meng and Kunze ${ }^{35}$ in 1979 to perform LIF in plasma. Hill et al. ${ }^{36}$ were the first to use the capability to tune the laser wavelength to obtain velocity selective LIF measurements in 1983. Instead of measuring the emission line shape, which is limited by the resolution of the spectrometer used for detection, they measured the absorption line shape with a velocity resolution determined by the natural linewidth of the laser transition. The dye laser was the primary laser choice for LIF for many years due to its high power output and useful wavelength range. Recently,, diode laser technology has improved significantly, yielding lasers in more wavelength ranges with increased power and tenability, and now LIF is routinely accomplished with either dye or diode lasers. A detailed review of LIF with tunable dye lasers can be found in Ref. [10] while a review of LIF with a tunable diode laser can be found in Ref. [13].

In a LIF measurement of a particle velocity distribution function (VDF) in plasma, the frequency of the narrow linewidth, tunable laser is scanned across an absorption line of an ion or neutral species in the plasma and the intensity of the fluorescent emission from the excited state is measured as a function of the laser frequency. Typically, only metastable excited states or ground states are populated enough to be used for the initial state. In the plasma, moving ions or neutrals absorb the Doppler shifted laser light in their rest frame, thereby pumping an electron to a higher energy, usually a short-lived, excited state, which then spontaneously decays. The fluorescent emissions is spatially limited to the width of the laser beam and with focused collection optics, the spatial resolution achieved in LIF can be as small as $1 \mathrm{~mm}^{3}$. The initial state density (with an absolutely calibrated system), ion or neutral temperature, and 
the net drift velocity can be determined from the frequency dependence of the measured absorption spectrum.

For a Maxwellian distribution of ion velocities, the Doppler broadening of an ion absorption line is

$$
I(v)=I_{0} \exp \left(\frac{-m\left(v-v_{0}^{*}\right)^{2}}{2 k_{B} T c^{2} v^{2}}\right)
$$

where $I(v)$ is the absorbed photon flux as a function of frequency $v, I_{0}$ is the maximum photon flux absorption and $v_{0}{ }^{*}=v_{0}+v_{0} \cdot \mathrm{v}_{0} / c$ is the average proper frequency of the transition when viewed from the laboratory frame. LIF is an indirect method of measuring this Doppler broadened absorption line since instead of directly measuring the absorption profile, the emission intensity from the excited state is monitored as a function of the injected laser frequency.

In addition to Doppler broadening, there are other mechanisms such as the natural line width, Stark broadening, power broadening and Zeeman broadening that affect the shape of the absorption profile. For typical experimental conditions in HELIX and LEIA, the only two significant line broadening mechanisms are Zeeman splitting and thermal Doppler broadening. ${ }^{37}$ In the presence of a strong magnetic field, Zeeman splitting yields linearly polarized $\pi$ lines $(\Delta m=0)$ and circularly polarized sigma $\sigma$ lines $(\Delta m= \pm 1)$ for absorption between the initial and the upper states. Fig. 3.14 (a) and (b) show the details of typical Zeeman splitting for the primary $611 \mathrm{~nm}$ and $668 \mathrm{~nm}$ absorption lines of argon, respectively. The $\pi$ lines are symmetrically distributed around the zero magnetic field transition. The $\sigma$ lines include two clusters of lines, $\sigma+$ and $\sigma-$. The amplitude envelope of each of $\sigma^{+}$or $\sigma$ - clusters is asymmetric, but each cluster is distributed symmetrically around the central line (zero magnetic field transition). The shift in frequency of each cluster from the central line depends linearly on the magnetic field strength. From the measured shift of the $\sigma$ clusters, the strength of the magnetic field at the measurement location can be determined. 


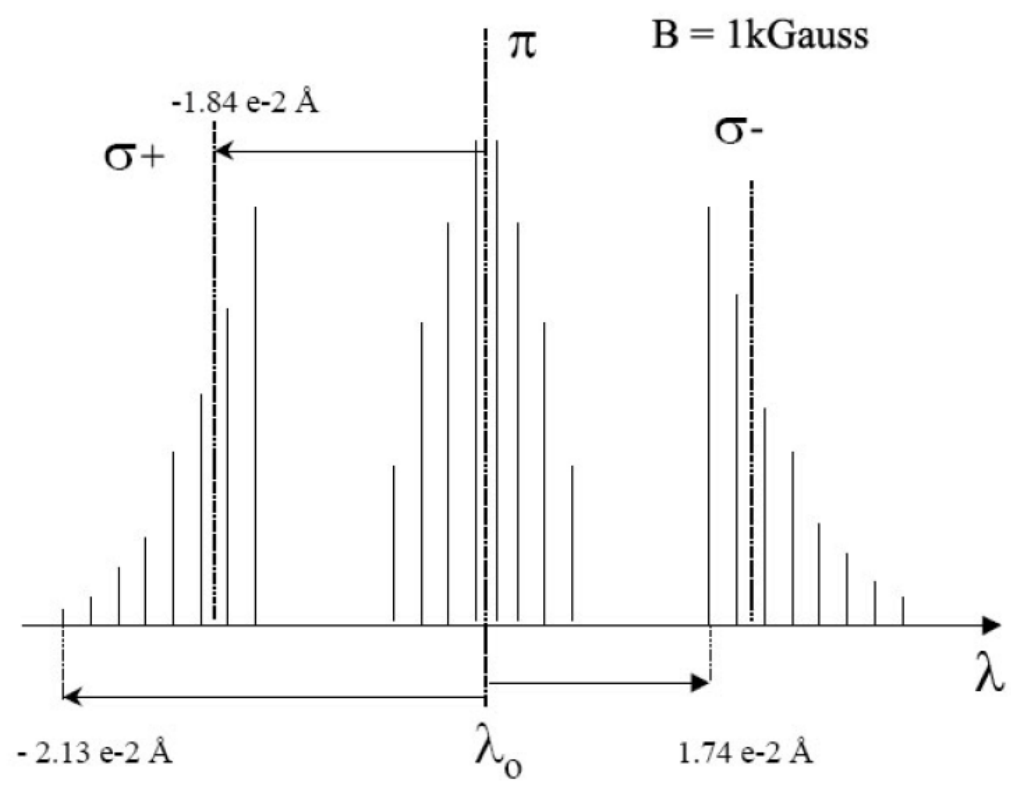

(a)

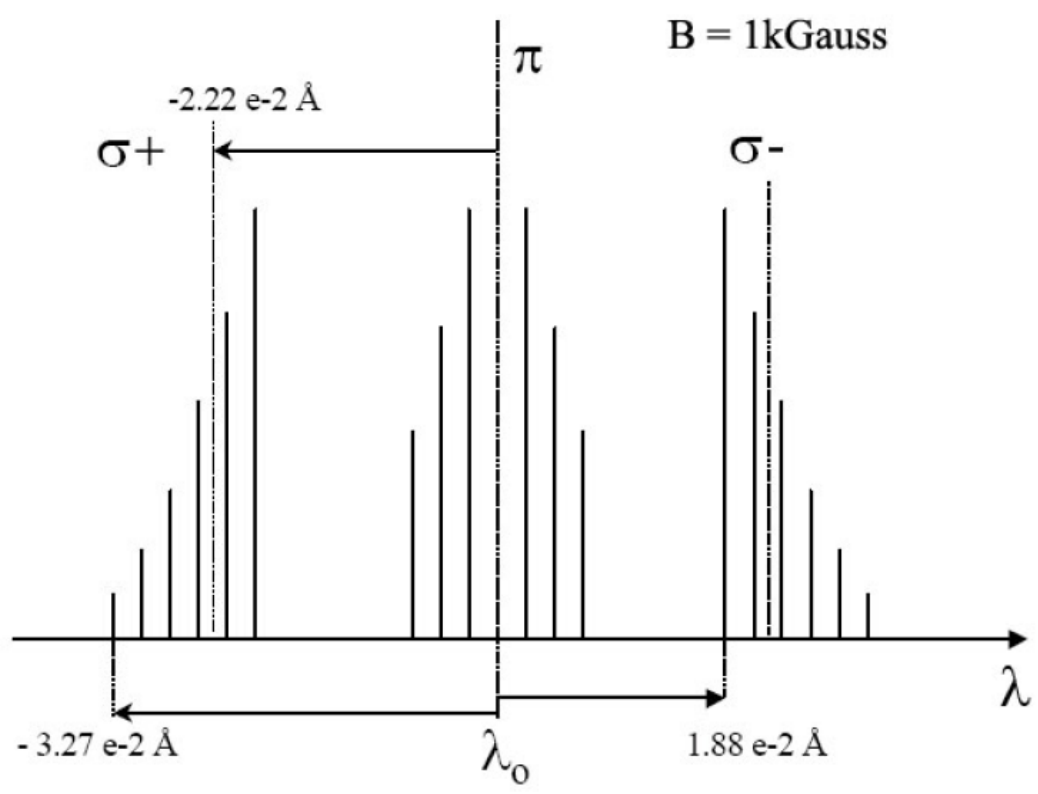

Figure 3.14. Schematic of the $\sigma$ and $\pi$ transitions for argon ion absorption lines. The height of each line corresponds to the statistical weighing of the each transition as a function of wavelength for the: (a) $611.6616 \mathrm{~nm}$ line and (b) $668.6138 \mathrm{~nm}$ line. The figure is adapted from Ref. [44]. 
For thermal particle distributions, each Zeeman component is also Doppler broadened. To get a true measurement of the temperature from the measured LIF signal, the signal has to be de-convolved into the individual Zeeman components. ${ }^{38}$ However, for typical LIF measurements in HELIX-LEIA, the Zeeman splitting of each of the two sets of $\sigma$ lines and the set of $\pi$ lines is much less than the Doppler broadening for each of the individual $\sigma$ and $\pi$ lines. ${ }^{44}$ In this case, the two sets of $\sigma$ lines can be treated as two Doppler broadened lines shifted from the proper wavelength by the statistically weighted average Zeeman shift of the individual $\sigma$ lines. Similarly, the $\pi$ lines can be treated as a single Doppler broadened line.

By suitable choice of laser injection relative to the magnetic field, either the parallel (to the magnetic field) or the perpendicular particle distributions can be measured. For perpendicular injection, the laser is polarized parallel to the magnetic field and only the $\pi$ lines are excited. For parallel injection, the laser is circularly polarized so that the $\sigma$ lines are excited. For relatively weak magnetic fields the two $\sigma$ clusters are not individually resolved in frequency and they broaden the measured absorption line.

\subsubsection{LIF Diagnostic Apparatus}

A representative LIF experimental set up is shown in Fig. 3.15 for an argon-ion pumped dye laser (for more details see Section 3.4.2). Experiments were also performed with a portable diode laser (for more details see Section 3.4.3). The laser first passes through a 90-10 beam splitter (BS). The 10\% of the laser light extracted goes through an iodine cell for an absolute zero velocity reference. Spontaneous emission from the iodine cell absorption lines is collected by a photodiode (PHD) for each scan of the laser wavelength. With the iodine cell as a reference, bulk ion speeds can be reliably resolved up to $25 \mathrm{~m} / \mathrm{s}$. A Burleigh WA-1500 high resolution wavemeter, placed after the iodine cell, and is used to monitor the laser wavelength during tuning of the. 


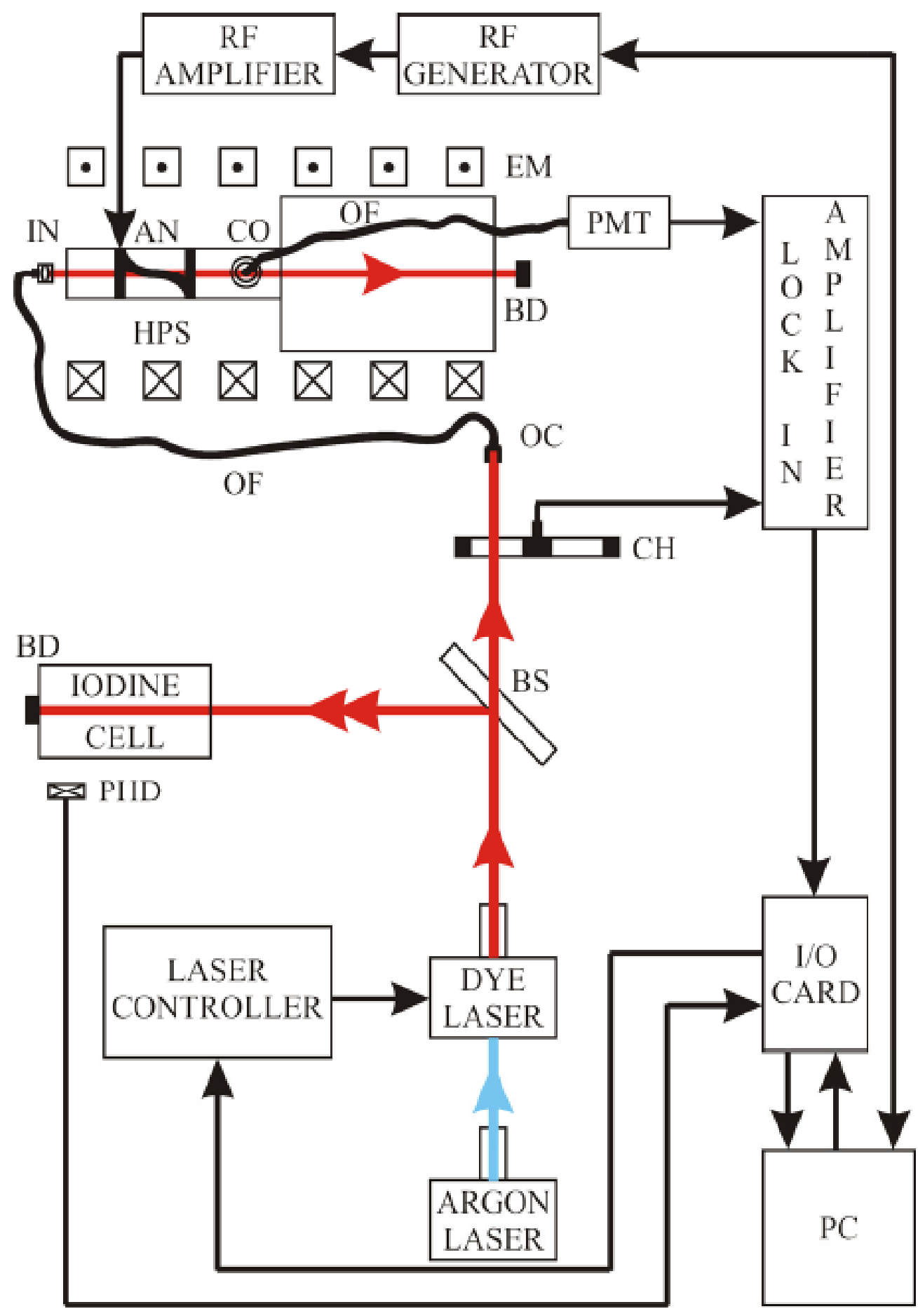

Figure 3.15. The LIF diagnostic apparatus: HPS - helicon plasma source; IN - injection optics; AN - antenna; CO - collection optics; BD - beam dump; EM - electro-magnets; $\mathrm{CH}$ - chopper; PMT - photo multiplier tube; OF - optical fiber; OC - optical coupler; PHD - photo diode and BS - beam splitter. Figure obtained from Ref. [11]. 
The remaining $90 \%$ of the laser light is modulated by an optical chopper $(\mathrm{CH})$ at a few kilohertz and coupled (OC) into a multi-mode, non-polarization preserving, optic fiber (OF) that transports the laser light to the experimental device. The parallel injection optics (IN) includes a combined linear polarizer and quarter-wave plate optical element that converts the unpolarized laser light into either right or left circularly polarized light (to pump only one of the two $\sigma$ clusters). The perpendicular injection optics includes a linear polarizer to select only laser light with polarization parallel to the magnetic field direction. The fluorescent light, together with the background light, is coupled into another fused silica optical fiber by the collection optics (CO). The fiber used has a numerical aperture (NA) of 0.22. For the maximum coupling of the collected light into a fiber using a lens with focal length $f$ and diameter $D$, the relationship $D / 2 f<\sin ^{-1}(N A)$ must be satisfied. For a 1” $(2.54 \mathrm{~cm})$ diameter lens, the focal length must be a little larger than $5 \mathrm{~cm}$. The intersection of the $5 \mathrm{~mm}$ diameter injected laser beam and the $0.8 \mathrm{~mm}$ diameter collection focal spot yields a very highly spatially resolved measurement volume of $\sim 4 \mathrm{~mm}^{3}$. The collected light is then transported to a filtered (bandwidth of 1 nm) photomultiplier tube (PMT). The Hamamatsu HC124-06 photomultiplier tube has an integrated $30 \mathrm{kHz}$ bandwidth pre-amplifier. A Stanford Research SR 830 lock-in amplifier is used to isolate the LIF signal from the intense background emission at the same wavelength. The chopper frequency serves as the reference to the lock-in amplifier which eliminates non-correlated signals at all other frequencies. Although the fluorescent signal is on the order of 1 in $10^{6}$ of the background light, typical LIF measurements using the chopper and lock-in amplifier combination gives a signal-to-noise ratio of 20:1.

\subsubsection{LIF with a Dye Laser}

For the LIF measurements using the dye laser in this work, light from a tunable ring dye laser was injected into an argon plasma to optically pump only those argon ions whose Doppler shifted absorption frequency matched the laser frequency. The dye laser consists of a $100 \mathrm{MHz}$ linewidth, continuous wave argon-ion pumped ring dye laser (a 6 Watt Coherent Innova 300 argon ion laser pumps a Coherent 899 ring dye laser using the Rhodamine 6G dye that fluoresces from $560 \mathrm{~nm}$ to $620 \mathrm{~nm}$ ). The absorption line chosen for singly excited argons ions (Ar II) is 611.4923nm (in air), which corresponds to 
$611.6616 \mathrm{~nm}$ (in vacuum). The laser light pumps the argon ions from the metastable state $3 d^{2} G_{9 / 2}$ to the higher state $4 p^{2} F_{7 / 2}$. An ion in the upper $4 p^{2} F_{7 / 2}$ state then decay to the $4 s^{2} \mathrm{D}_{5 / 2}$ state by emitting a photon of wavelength $461.086 \mathrm{~nm}$. The LIF scheme is shown in Figure 3.16.
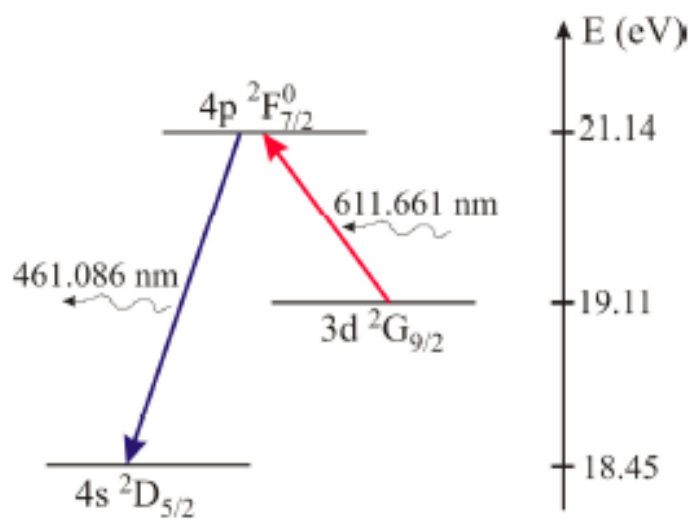

Figure 3.16. LIF scheme for Ar II using the ring dye laser.

To accurately determine the ion velocity, ion temperature and the ion density from the LIF measured IVDF, the LIF system must be calibrated in absolute laser frequency. For absolute metastable density measurements, a full calibration of the efficiencies of all the optical elements is required. Although the resolution of the Burleigh 1800 wavemeter is sufficient to tune the ring dye laser to the correct wavelength, the wavemeter refresh rate is too slow to keep up with the laser scanning rates used in typical LIF measurements. Therefore, an iodine absorption spectrum was recorded for each scan of the laser. To identify the appropriate iodine lines to be used as a zero velocity reference for the LIF measurements, the Salami ${ }^{39}$ reference iodine spectrum was compared to the experimentally obtained iodine spectra in the ranges of interest of each LIF scheme. Typically, the dye laser frequency was scanned over $20 \mathrm{GHz}$ for each measurement. For scans wider than $20 \mathrm{GHz}$, individual $20 \mathrm{GHz}$ scans were stitched together.. As shown by the arrow in Fig. 3.17, for the Ar II absorption line at 661.6616 $\mathrm{nm}\left(16348.91 \mathrm{~cm}^{-1}\right)$, the iodine line closest to the zero velocity reference with a sufficiently strong intensity is the $16348.94 \mathrm{~cm}^{-1}$ line, which correspond to a shift of 1.08 GHz. For each measurement, the bulk velocity of the Ar II IVDF is determined from 


$$
V \cong \lambda_{0} \Delta v_{\text {total }}
$$

where the velocity $V$ is in $\mathrm{m} / \mathrm{s}$ if the rest frame wavelength $\lambda_{0}$ is in $\mathrm{nm}$ and the absolute frequency shift $\Delta v_{\text {total }}$ is in $\mathrm{GHz}$.

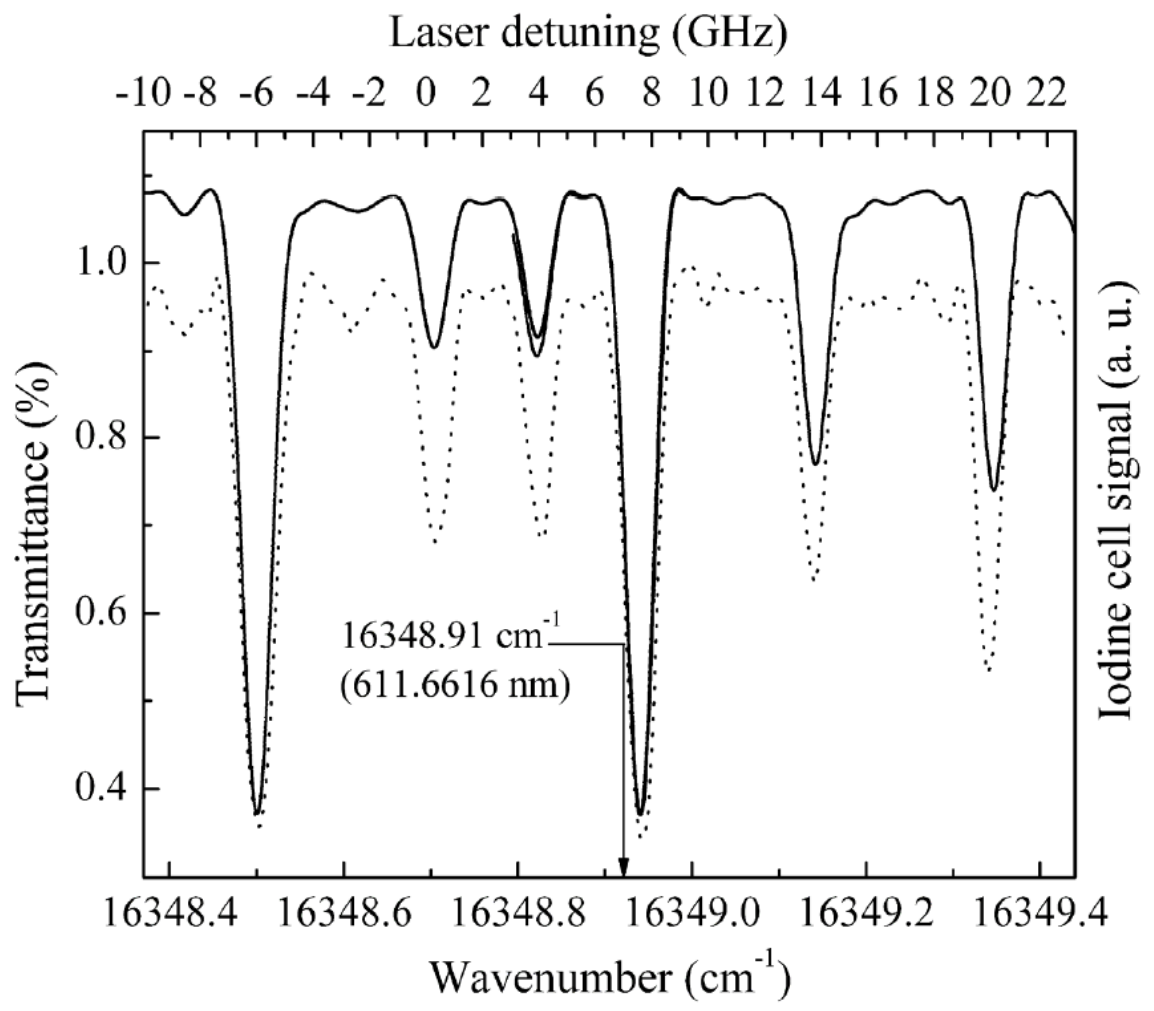

Figure 3.17. Iodine spectra for approximately $\pm 15 \mathrm{GHz}$ relative to $611.6616 \mathrm{~nm}$. This spectrum obtained from two partially overlapping $20 \mathrm{GHz}$ wide laser scans (solid line). The iodine spectrum in the spectral ranges of interest for Ar II, as given in Ref. [39], is shown as the dotted line. Figure adapted from Ref. (11).

Figure 3.18 shows a typical LIF measurement of an Ar II IVDF obtained in LEIA, $126 \mathrm{~cm}$ downstream of the antenna (position D in Fig. 2.3). The bulk velocity is calculated according to Eqn. 3.28 for the total frequency difference equal to difference between the LIF signal peak and the iodine reference line (1.46 GHz in this example) plus the $1.08 \mathrm{GHz}$ shift in the peak of iodine reference line from the unshifted absorption 
line at $611.6616 \mathrm{~nm}$ and minus the Zeeman shift due to the magnetic field at that position (this is a $\sigma^{+}$Zeeman line and for these experimental conditions, the Zeeman shift is 1.03 $\mathrm{GHz}$ ). The total frequency shift is $1.51 \mathrm{GHz}$, corresponding to a bulk velocity of $\sim 925$ $\mathrm{m} / \mathrm{s}$. The argon IVDF is well fit with a single Gaussian function, according to Eqn. 3. 26. From the full width at half maximum, the parallel ion temperature is determined to be $0.16 \mathrm{eV}$.

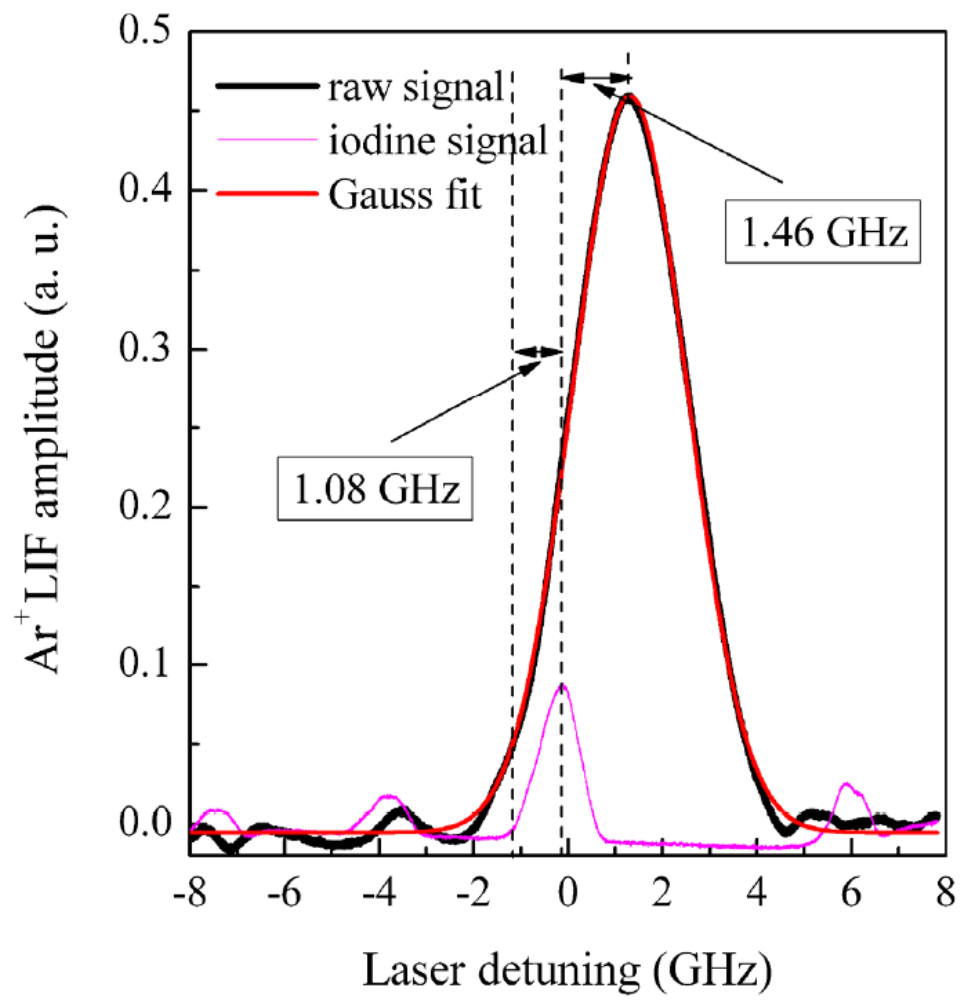

Figure 3.18. Typical LIF profile of argon IVDF, measured in HELIX, $126 \mathrm{~cm}$ from antenna (position D in Fig. 2.3). The black line is the raw LIF signal, the red line is a single Gaussian fit to the data, and the purple line is the iodine reference spectrum. This figure was obtained from Ref. [11].

\subsubsection{LIF with a Diode Laser}

Even though the diode laser was invented before the dye laser, ${ }^{40}$ the non-linearity of laser output intensity with injection current, unstable spatial profiles, requirement of liquid nitrogen temperatures, and widely spread cavity modes prevented diode lasers 
from being used for LIF. In recent years, commercially available diode lasers have overcome these challenges and are in widespread use in the spectroscopic research community. Modern diode lasers have several advantages over their $\mathrm{cw}$ dye laser counterparts: they are relatively inexpensive, they do not need high power electrical systems or water cooling, they are capable of a few gigahertz modulations, and being much smaller in size - they are portable. Diode lasers are now available from ultraviolet to infrared wavelengths; a wider range of wavelengths than achievable with a dye laser.

A typical laser diode consists of several semiconductor layers. The light is generated by sending a current (commonly known as "injection current") through the active region of the diode located between the $n$ and $p$ type cladding layers. The electrons and holes produced due to the injected current recombine to emit the light. The photon energy (and thus the wavelength of the laser) is determined by the band gap in the semiconductor due to the difference in the electron energy in the conduction band and the hole energy in the valence band. Diode lasers that emit in the $670 \mathrm{~nm}$ wavelength region are made from InGaA1P semiconductor. Typically, the semiconductors are doped to achieve the desired wavelength. In contrast to the gas lasers, the excited state (conduction band) and the ground state (valence band) are continuous energy bands for optical transitions in semiconductors. The wavelength dependence of the laser medium gain curve and the optical path length of the cavity, both depend on temperature. Fine tuning the wavelength of the laser is accomplished by changing the diode temperature (increasing the diode temperature shifts the cavity modes and the wavelength at the maximum gain towards higher wavelengths), or by changing the characteristics of the external resonant cavity that surrounds the laser diode.

Diode lasers are not without challenges. The shifting of the gain curve by changing the temperature of the laser causes jumps in wavelength from one longitudinal cavity mode to another. ${ }^{41}$ The emitted laser intensity is a strong function of the temperature of the laser (increasing the temperature increases the threshold value of the current for lasing, thus reducing the laser intensity at a fixed injection current) and hence temperature scanning often leads to unacceptable variations in the laser output power during a wavelength scan. Changing the injection current also changes the wavelength of the laser, primarily by indirectly changing the laser temperature by Joule heating, but also 
by changing the index of refraction through changes in the carrier charge density. ${ }^{41}$ Since laser power also depends on the laser current, injection current modification is not a practical means to performing a wavelength scan. Thus external cavity tuning is preferred for fine tuning a laser. The external cavity also helps to narrow the linewidth of the laser. $^{41}$

An external cavity can be made by using an anti-reflection coating on the diode along with external optical components to produce a laser resonator. A pseudo-external cavity set up has a diode with only one facet with an anti-reflection coating. Two commonly used pseudo-external cavity configurations are the Littman/Metcalf ${ }^{42}$ and the Littrow $^{43}$ configuration (shown in Fig. 3.19).

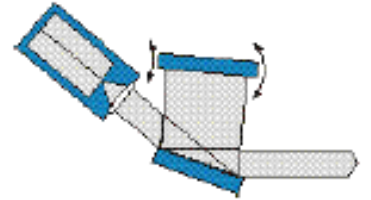

(a)

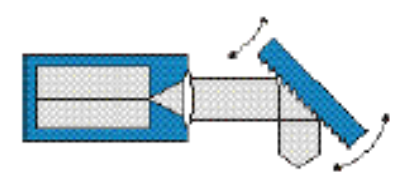

(b)

Figure 3.19. Two commonly used diode laser external cavity resonators. (a) Littman/Metcalf configuration. (b) Littrow configuration. The figure is adapted from Ref. [13].

In the Littman/Metcalf configuration, output from the laser is directed towards a grating at grazing incidence. The diffracted light reflects off a tuning mirror back to the grating. Wavelength tuning is achieved by rotating this tuning mirror. In a Littrow configuration, wavelength tuning is achieved by changing the angle of the external diffraction grating. The grating is placed at the Littrow angle, defined such that the first order diffracted beam is coincident with the input beam. The zeroth-order beam reflects off the grating and is directed out of the cavity. The Littrow cavity offers more output power at the expense of tuning range. The Littman cavity is a double pass grazing incidence cavity that naturally achieves multi-mode suppression. On the other hand, the power in the Littrow configuration is much greater because the zeroth-order beam is lost for the Littman/Metcalf configuration. Because of the crucial importance of laser power for LIF measurements, we chose a Littrow cavity diode laser for our original diode laser based LIF measurements using low power diode lasers. 
Previously we had used two different diode lasers made by Sacher Lasertechnik. The first diode laser used was SAL-665-10, which had a $1.5 \mathrm{MHz}$ bandwidth, $15 \mathrm{mWatt}$ maximum output power and a mode-hop free tuning range of $\sim 14 \mathrm{GHz}(\sim .021 \mathrm{~nm}) .{ }^{44}$ That laser was later upgraded to a SAL-665-20, another Littrow mounted diode laser with a mode-hop-free tuning range of $20 \mathrm{GHz}$ and an increased maximum output power of 20 mWatt. Unfortunately, in May 2005, an electromagnetic pulse (EMP) in the lab destroyed that diode, which was subsequently replaced with a SAL-670-15, with a mode-hop-free tuning range of $10 \mathrm{GHz}$ and maximum output power of $15 \mathrm{mWatt}$. This last diode had terrible performance characteristics and its shortcomings are discussed in detail in Ref. [13]. With time, the diode's performance degraded even further and in 2009 the maximum output power was $\sim 7 \mathrm{~mW}$ att and finding a reasonably wide mode-hop-free tuning range was very difficult. The destruction of the diode laser by the EMP demonstrates their extreme sensitivity to environmental conditions. To safeguard the diodes an uninterrupted power supply (UPS) is now used for the laser controller, the laser injection current is turned on and off in gradual steps, and a wrist grounding strap attached to the laser table is worn at all times when working with the laser.

The new tunable diode laser system used for the experiments in this work was manufactured by Toptica Photonics (Model TA 100) and consists of a master oscillator followed by a tapered amplifier; a MOPA (master oscillator power amplifier) configuration..

Typically free-running laser diodes have a linewidth of about $100 \mathrm{MHz}$. However, using optical feedback from the external cavity, the laser linewidth of the master oscillator is reduced to $\sim 1 \mathrm{MHz}$. The laser light emitted from the front facet of the laser diode is collimated by a lens with a very short, diffraction-limited, focal length and then hits the reflection grating. The grating is mounted in the Littrow configuration. The first order diffraction beam reflected back passes through the collimator again and is focused onto the laser diode resonator. As the feedback of the grating is considerably higher than the feedback from the front facet, the laser resonator consisting of the rear facet and the reflection grating forms an external oscillator. The free spectral range of this new resonator, which is only a few centimeters long, is considerably less than that of the 
individual laser diode and has a higher finesse. The result is a very narrow laser linewidth.

To achieve more output power than is possible with even the most powerful single mode laser diode, the collimated output beam of the master oscillator is focused onto the entrance facet of a tapered amplifier (amplifier with a tapered gain region; see Fig. 3.20). The amplifier has anti-reflecting coatings on both facets to prevent laser emission without seeding. Thus, the internal modes of the amplifier are completely controlled by the master oscillator. Because of a broad gain profile, the tapered amplifiers can achieve a relatively large coarse tuning range about their center wavelength (the Toptica diode laser can be tuned from $663 \mathrm{~nm}$ to $673 \mathrm{~nm}$ ) with a very high output power (up to 500 mWatts).

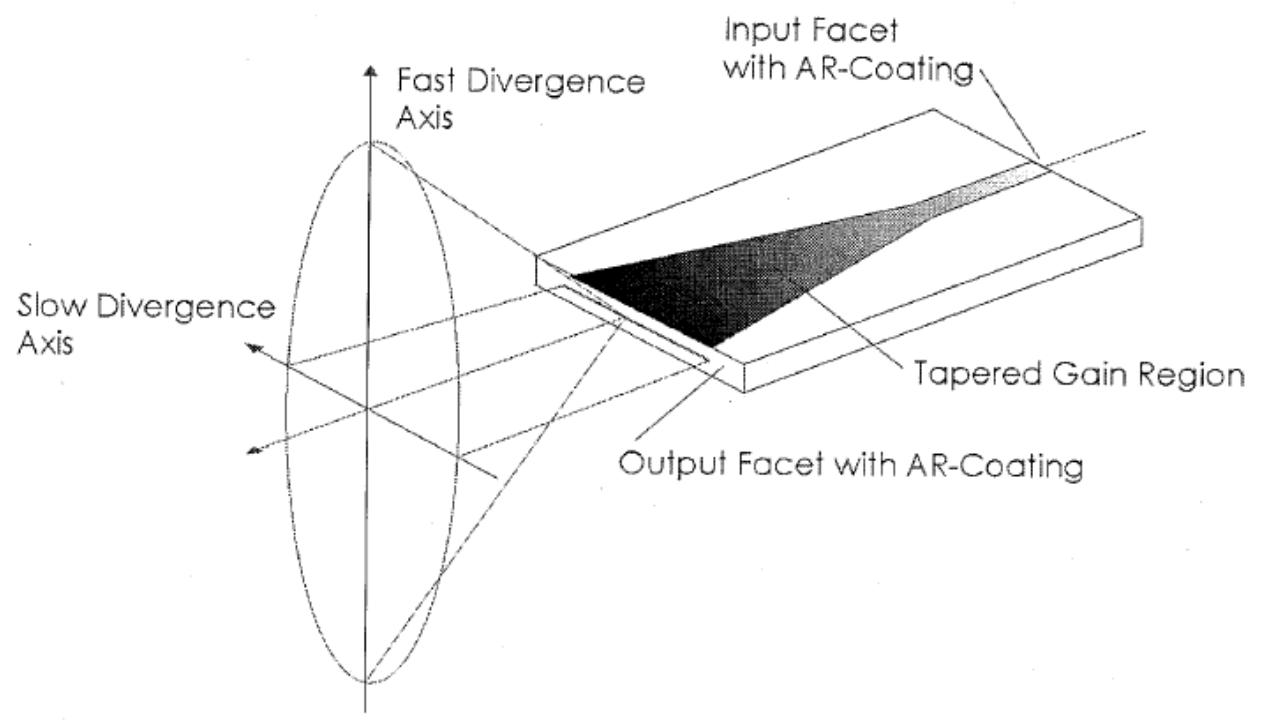

Figure 3.20. A representative diagram showing the tapered amplifier region and non-Gaussian beam characteristics of a typical diode laser.

The spatial beam profile of a typical tapered amplifier output is anisotropic. The beam has a high divergence in the direction perpendicular to the elongated direction of the output facet and a small divergence in the parallel direction. For a well collimated output beam, the beam cross section is elliptical (3:1). To achieve a more circular beam 
spot, the high direction axis is collimated with a very high numerical aperture collimator in front of the amplifier facet and the small divergence axis is corrected by a cylindrical lens afterwards. Still, the output beam is non-Gaussian and for experimental purposes where a single mode Gaussian beam is desirable, proper external beam shaping is necessary (described in Section 6.2.1). Because of the very high feedback sensitivity of both the master oscillator and the tapered amplifier, both the laser diodes are protected from reflections with very high extinction optical isolators (> $60 \mathrm{~dB}$ ).

In the Littrow configuration, the external cavity has two elements that determine the laser wavelength: the cavity length and the grating. Typically the cavity length is fixed and the grating angle is varied by changing the voltage on the piezoelectric stack (PZT) attached to the grating housing. The grating is mounted at the Littrow angle which is given by

$$
\sin \alpha=\frac{k \lambda}{2 d},
$$

where the angle $\alpha$ is given with respect to the normal to the grating, $k$ is the diffraction order (in our case, $k=1$ ), $\lambda$ is the wavelength of the laser and $d$ is the grating constant (depends on the number of lines per unit length on the grating). At $670 \mathrm{~nm}$, the line density of the grating used is 2200 lines/mm which gives a grating constant of $455 \mathrm{~nm}$. The deflection angle of $2 \alpha$ in that case is 94.95 degrees. The PZT integrated in the grating holder is a low voltage piezo-actuator controlled by a voltage range of $-5 \mathrm{~V}$ to $150 \mathrm{~V}$. It has a typical linear extension of 5 microns/150V. Rotating the grating does result in slight length adjustments to the external cavity length and to achieve a mode-hop-free wavelength scan, the grating and the cavity length must be tuned synchronously. For improved mode-hop-free wavelength tuning range, a simultaneous additional modulation of the laser diode current is used. Using an internal potential divider circuit, a small portion of the ramp voltage used to modulate the PZT for scanning is fed back into the power applied to the laser diode. This is commonly known as "feed forward" and only proper use of this feature ensures mode-hop-free wavelength tuning.

Figure 3.21 shows the effect of diode injection current on the emitted wavelength. The red line at 668.614 is the chosen wavelength corresponding to the 
absorption feature of Ar II, about which the PZT was manually scanned over a range of $10 \mathrm{GHz}(\sim 0.014 \mathrm{~nm})$. Figure 3.22 shows the typical laser power output as the amplifier current input is varied.

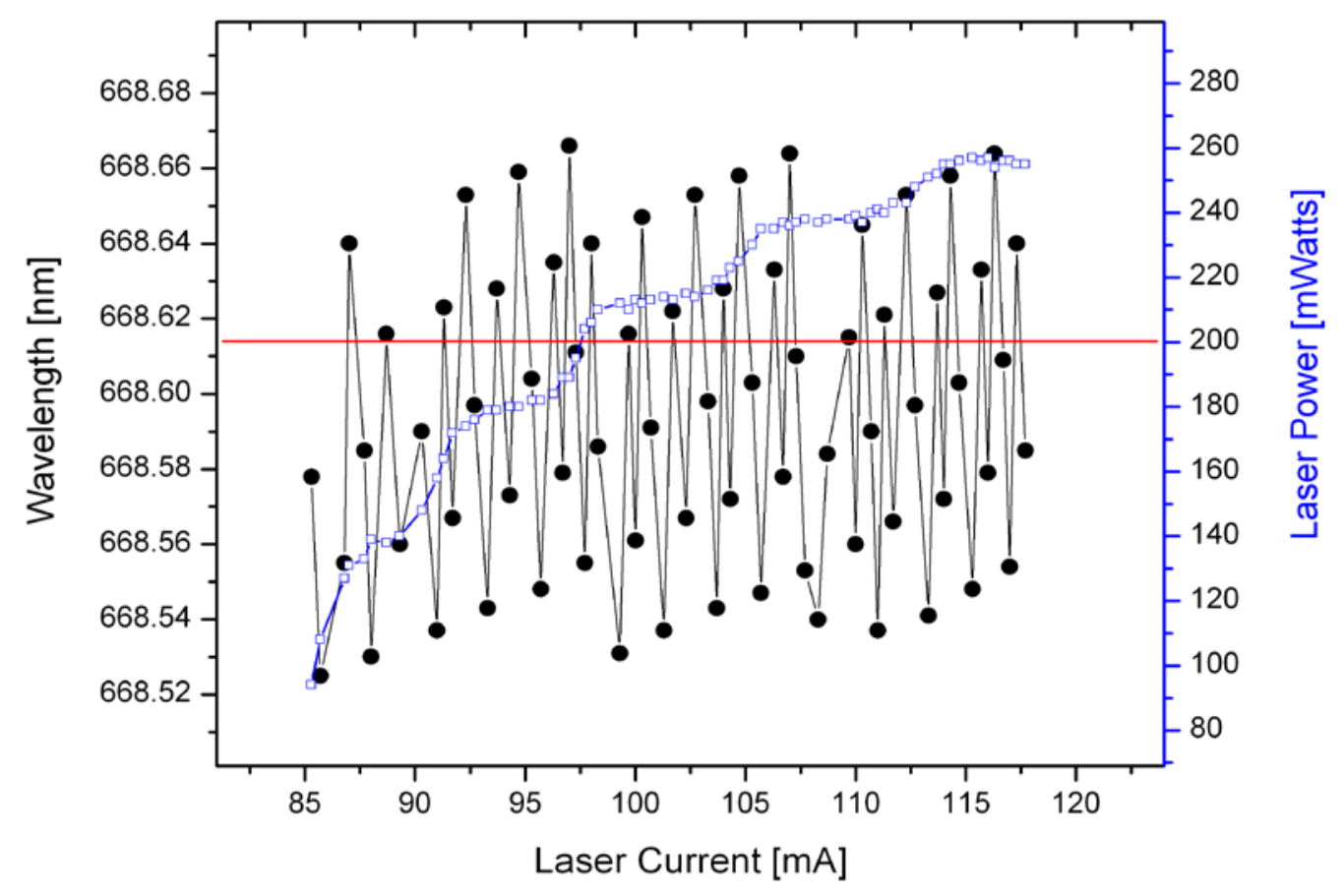

Figure 3.21. Diode laser wavelengths and output power versus injection current.

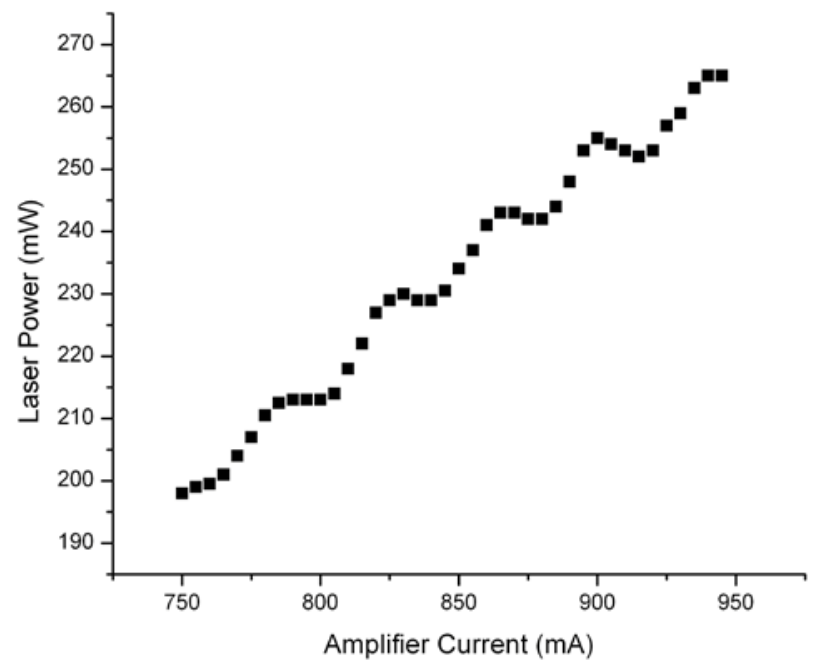

Figure 3.22. Laser output power versus tapered amplifier current. 
The absorption line chosen for diode laser LIF of singly excited argons ions (Ar II) is $668.6138 \mathrm{~nm}$ (in vacuum). The single mode, narrow band, output from the diode laser pumps the argon ions from the metastable state $3 \mathrm{~d}^{4} \mathrm{~F}_{7 / 2}$ to the upper state $4 \mathrm{p}^{4} \mathrm{D}_{5 / 2}$. Ions in the upper $4 \mathrm{p}^{2} \mathrm{~F}_{7 / 2}$ state then decay to a lower $4 \mathrm{~s}^{4} \mathrm{P}_{3 / 2}$ state, by emitting photons of wavelength $442.7244 \mathrm{~nm}$. The LIF scheme is shown in Figure 3.23.

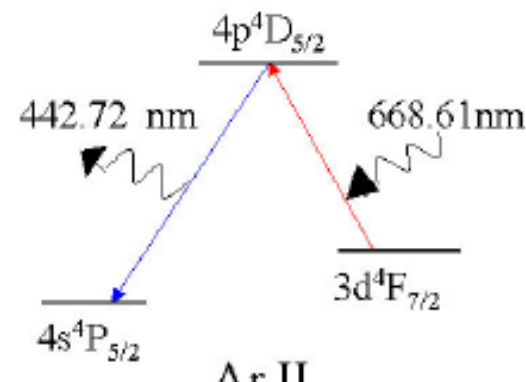

Figure 3.23. LIF scheme for Ar II using the diode laser.

\subsection{Scanning Internal Probes}

To measure plasma parameters at different spatial positions inside LEIA, two scanning internal probes were used. The super probe (see section 3.5.1) is capable of obtaining spatially resolved measurements in LEIA throughout a horizontal plane $100 \mathrm{~cm}$ in length along the z-axis and $40 \mathrm{~cm}$ wide in the radial direction (y-axis). The other scanning internal probe is the re-entrant probe (see section 3.5.2) that enters LEIA from the side opposite to that of the super probe and the probe tip can be placed at any location with a rotatable vacuum feedthrough.

\subsubsection{Super Probe}

Figure 3.24 shows the typical experimental geometry for the super probe. The measurement area begins in the divergent magnetic field region near the HELIX-LEIA junction and extends to the middle of the LEIA chamber. 


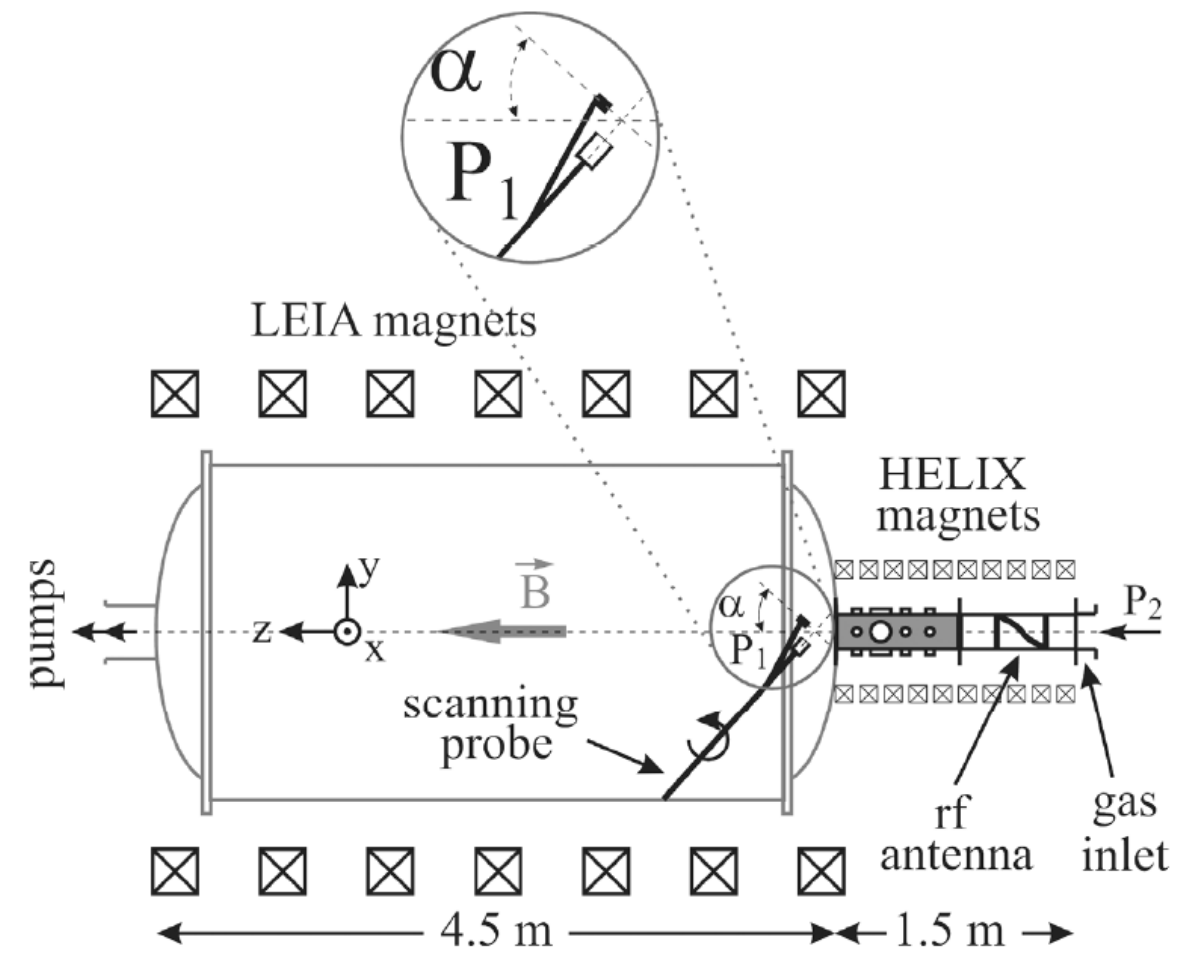

Figure 3.24. Top view of the HELIX-LEIA system showing the typical orientation of the super probe. The magnified insert shows the details of the injection geometry. Figure is from Ref. [11].

The backbone of the super probe is a 6' long, 3/4”- o.d. stainless steel shaft with 0.083”-thick walls supported by a stainless steel ball joint bearing mounted on the interior of a feedthrough flange, which is mounted on flexible welded bellows coupled to the LEIA chamber. Details of the probe construction can be found in Ref. [45]. The probe head comprises of LIF injection and collection optics, a rf-compensated cylindrical Langmuir probe and a 3-D magnetic sense coil array (see Figure 3.25). Motion along the $\mathrm{y}$-axis $(40 \mathrm{~cm})$ and the $\mathrm{z}$-axis $(100 \mathrm{~cm})$ is achieved by two computer-driven VELMEX ${ }^{\mathrm{TM}}$ stepping motor assemblies that control the insertion depth of the probe and the tilt angle between the probe and the chamber axis. A VELMEX ${ }^{\mathrm{TM}}$ rotary stepping motor spins the probe shaft about its axis to switch between parallel or perpendicular LIF measurements (with respect to the magnetic field) and for optical tomography. This design keeps the LIF investigated plasma volume (the intersection between the plasma volume and the collection optics field of view) the same for all injection angles. The spatial and angular resolutions are determined by the precision of the stepping motors and are $\sim 1 \mathrm{~mm}$ and $~$ 
$0.5^{\circ}$ respectively. When inserted into the plasma, the LIF interrogation volume is upstream of the Langmuir probe, which is upstream of the magnetic sense coil array. Thus, the most perturbative component of the probe, the magnetic sense coil array, is the last part of the probe to interact with the plasma flowing out of HELIX.

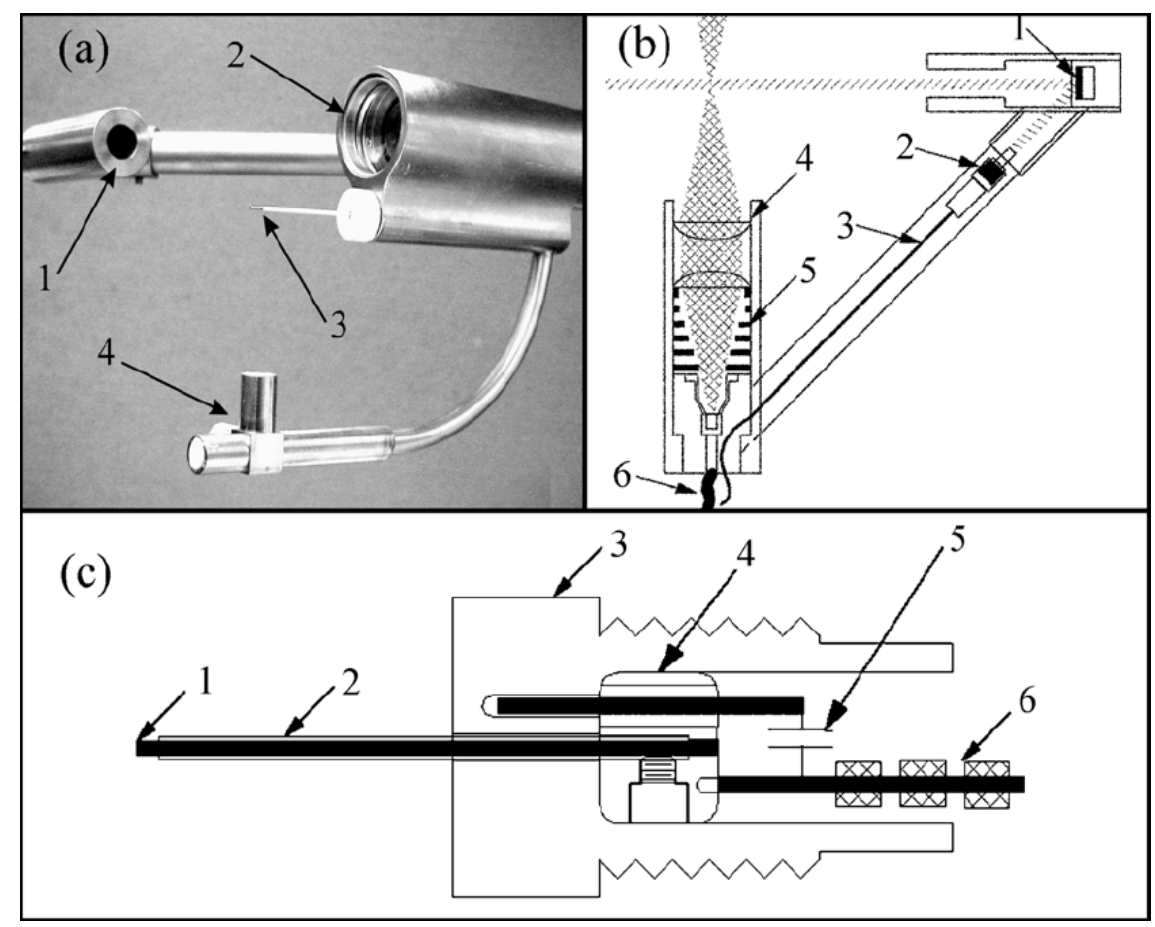

Figure 3.25. Super probe head diagnostics: (a) 1 - LIF injection optics, 2 - LIF collection optics, 3 - rf compensated Langmuir probe, 4 - 3D magnetic sense coil array; (b) 1 - injection mirror, 2 collimating injection optics, 3 - injection fiber; 4 - collection lens, 5 - light baffles; 6 - collection fiber;,(c) 1 - 0.5 mm graphite rod, 2 - alumina tube, 3 - boron nitride cap, 4 - brass plug, 5 - 10nF shorting capacitor and 6 - rf choke chain. The Figure is adapted from Ref. [44].

For LIF measurements using the super probe, the laser light is coupled into the internal 200 micron fused silica fiber through a fiber-fiber vacuum feedthrough. The injection fiber is terminated by a $1 / 4$ " collimating lens to create a weakly divergent beam that reflects from a plane mirror and passes $5 \mathrm{~cm}$ in front of the collection optics (see Fig. 3.25 (a)). The 1" diameter collection optics consists of a $5 \mathrm{~cm}$ focal length collection lens followed by a $5 \mathrm{~cm}$ focal length focusing lens. The numerical aperture of the focusing lens was chosen to match the numerical aperture of the $1 \mathrm{~mm}$ core diameter, $2 \mathrm{~m}$ long 
fused silica collection fiber ( $\mathrm{NA}=0.22$ ). Between the focusing lens and the collection fiber, a series of circular light baffles prevent off axis rays from passing through the lenses into the collection fiber. Since the plasma strongly emits at the fluorescence wavelength, reduction of background light is critical for improved signal-to noise ratio. To avoid loss of fluorescence light at another fiber-fiber vacuum feed through, the collected light is directly coupled into a Hamamatsu HC-124-06 PMT, mounted on the end of the probe shaft. The collected fluorescence radiation is collimated inside the probe, passes through a standard quartz fused silica window, a Dell Optics 1-nm wide bandpass filter (centered on $461 \mathrm{~nm}$ for Ar II LIF) and into the PMT. The PMT moves with the probe as it scans through the measurement plane in LEIA. Due to the losses at each optical interface and in the fibers, only about $40 \%$ of the laser power is effectively injected into the plasma. The parallel IVDF can be measured either "actively", by injecting the laser from and collecting the fluorescence with the super probe head, or "passively" by injecting the laser parallel along the axis of the chamber from the HELIX end and collecting with the super probe head.

To measure the plasma density, electron temperature and the floating potential in LEIA, the super probe head includes a rf compensated Langmuir probe. The Langmuir probe design on the super probe is the same as described in Section 3.1.2.

\subsubsection{Re-entrant Probe}

In addition to the super probe feedthrough assembly, another rotatable feedthrough was constructed for use with Langmuir and LIF collection probes in LEIA (see Fig. 3.26). The feedthrough design was based on the schematics available in Ref. [46]. For compatibility with existing probe hardware at WVU, the feedthrough was modified to use a QF-40 flange instead of the original design for QF-50. The ball, sealed with two O-rings, enables angular motion of the probe and linear motion is provided by a double O-ring seal that is connected to the end of the ball by the QF-40. 


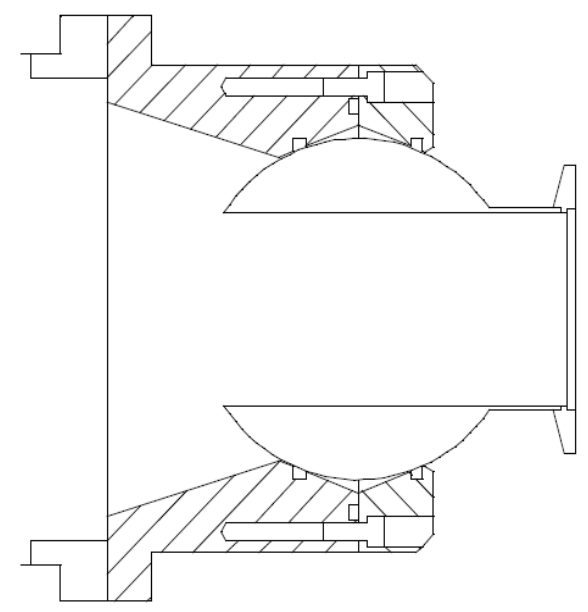

Figure 3.26. Mechanical drawing of the UCLA tilting port, modified for use at WVU. Figure is adapted from Ref. [44].

This rotatable feedthrough is used for inserting a Langmuir probe or for collection optics for LIF measurements in LEIA. The placement of the feedthrough provides better access to the HELIX-LEIA junction region than the super probe. Fig. 3.27 shows the design of the collection optics in the re-entrant probe. This probe contains miniature collection optics in a glass tube inserted into the plasma through the rotatable feedthrough. The laser is injected from the HELIX end of the machine, parallel to the axis of the chamber, and the fluorescent emission is collected by the re-entrant probe to obtain parallel measurements of the IVDF. Emission from the $461 \mathrm{~nm}$ line is focused into the fiber with a $1 / 4$ " plano-convex lens. The fiber is then connected to the same PMT used for typical LIF measurements in HELIX.

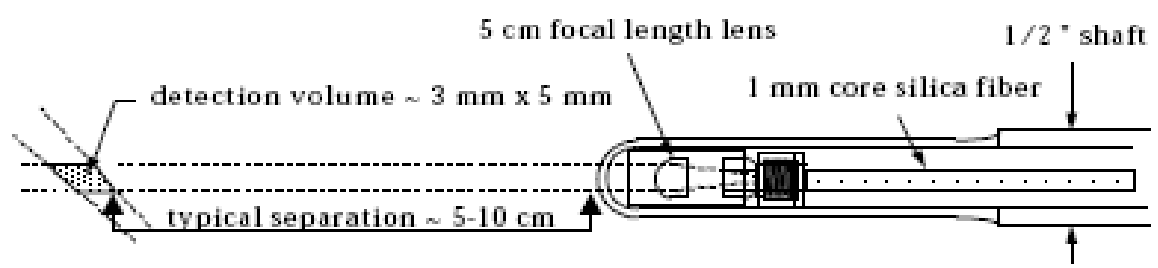

Figure 3.27. Schematic of the re-entrant probe. Figure adapted from Ref. [14]. 


\section{Chapter 3 References}

${ }^{1}$ I. Langmuir, Phys. Rev. 28, 727 (1926)

${ }^{2}$ F. F. Chen, in Plasma Diagnostic Techniques, ed. by R. H. Huddlestone and S. L. Leonard (Academic Press, New York, 1965)

${ }^{3}$ L. Schott, in Plasma Diagnostics, ed. by W. Lochte-Holtgreven (John Wiley, New York, 1968)

${ }^{4}$ I. H. Hutchinson, Principles of Plasma Diagnostics, (Cambridge University Press, 1987)

${ }^{5}$ N. Hershkowitz, How Langmuir Probes Work, in Plasma Diagnostics, Discharge Parameters and Chemistry, ed. by O.Aucillo and. D.L. Flamm (Academic Press, 1989)

${ }^{6}$ V. I. Demidov, S. V. Ratynskaia and K. Rypdal, Rev. Sci. Intrum. 73, 3409 (2002)

${ }^{7}$ P. A. Keiter, E. E. Scime, M. M. Balkey, R. Boivin, J. L. Kline and S. P. Gary, Phys. Plasmas 7, $779(2000)$

${ }^{8}$ N. A. Krall and A. W. Trivelpiece, Principles of Plasma Physics, (San Francisco Press, 1986)

${ }^{9}$ J. C. Sprott, Electrostatic Probe Techniques, PLP 88, University of Wisconsin, Madison (1966)

${ }^{10} \mathrm{~J}$. L. Kline, Slow Wave Ion Heating and Parametric Instabilities in the HELIX Helicon Source, $\mathrm{Ph}$. D. Thesis, West Virginia University, Morgantown (2002)

${ }^{11}$ I. A. Biloiu, Laser Induced Fluorescence Studies of Ion Acceleration in Single and Multiple Species Expanding Plasmas, Ph. D. Thesis, West Virginia University, Morgantown (2009)

${ }^{12}$ I. D. Sudit and F. F. Chen, Plasma Sources Sci. Technol. 3, 162 (1994)

${ }^{13}$ A. M. Keesee, Neutral Density Profiles in Argon Helicon Plasmas, Ph. D. Thesis, West Virginia University, Morgantown (2005)

${ }^{14}$ P.A. Keiter, Experimental Investigation of Ion Temperature Anisotropy Driven Instabilities in a High Beta Plasma, Ph. D. Thesis, West Virginia University, Morgantown (1999)

${ }^{15}$ M. J. Burin, G. R. Tynan, G. Y. Antar, N. A. Crocker and C. Holland, Phys. Plasmas 12, 052320 (2005)

${ }^{16}$ R. A. Hardin, Measurement of Short-Wavelength Electrostatic Fluctuations in a Helicon Plasma Source, Ph. D. Thesis, West Virginia University, Morgantown (2008)

${ }^{17}$ J. M. Beall, Y. C. Kim and E. J. Powers, J. Appl. Phys. 53, 3933 (1982)

${ }^{18}$ J. W. Coburn and E. Kay, J. Appl. Phys. 43, 4965 (1972)

${ }^{19}$ K. Kohler, J. W. Coburn, D. E. Horne, E. Kay and J. H. Keller, J. Appl. Phys. 57, 59 (1985)

${ }^{20}$ S. G. Ingram and N. S. J. Braithwaite, J. Phys. D. 21, 1496 (1988)

${ }^{21}$ A. D. Kuypers and H. J. Hopman, J. Appl. Phys. 67, 1229 (1990)

${ }^{22}$ W. M. Holber and J. Forster, J. Vac. Sci. Technol. A 8, 3720 (1990)

${ }^{23}$ J. Janes and C. Huth, Appl. Phys. Lett. 61, 261 (1992) 
${ }^{24}$ C. Charles, R. W. Boswell and R. K. Porteous, J. Vac. Sci. Technol. A 10, 398 (1992)

${ }^{25}$ C. Bohm and J. Perrin, Rev. Sci. Instrum. 64, 31 (1993)

${ }^{26}$ J. R. Woodworth, M. E. Riley, P. A. Miller, C. A. Nichols and T. W. Hamilton, J. Vac. Sci. Technol. A 15, 1 (1997)

${ }^{27}$ E. A. Edelberg, A. J. Perry, N. Benjamin and A. S. Aydil, Rev. Sci. Instrum. 70, 2689 (1999)

${ }^{28}$ D. Gahan, B. Dolinaj and M. B. Hopkins, Rev. Sci. Instrum. 79, 033502 (2008)

${ }^{29}$ C. Charles, A. W. Degeling, T. E. Sheridan, J. H. Harris, M. A. Leiberman and R. W. Boswell, Phys. Plasmas 7, 5232 (2000)

${ }^{30}$ F. Valsaque, G. Manfredi, J. P. Gunn and E. Gauthier, Phys. Plasmas 9, 1806 (2002)

${ }^{31}$ A. von Angel, Ionized Gases (Oxford University Press, London, 1965)

${ }^{32}$ Z. Harvey, S. Chakraborty Thakur, A. Hansen, R. A. Hardin, W. S. Przybysz and E. E. Scime, Rev. Sci. Instrum. 79, 10F314 (2008)

${ }^{33}$ Y.T. Yardley and C. B. Moore, Journal of Chemical Physics 45, 1066 (1966)

${ }^{34}$ R. A. Stern and J. A. Johnson, Phys. Rev. Lett. 34, 1548 (1975)

${ }^{35}$ H. C. Meng and H. J. Kunze, Phys. Fluids 22, 1082 (1979)

${ }^{36}$ D. N. Hill, S. Fornaca and M. G. Wikham, Rev. Sci. Instrum. 54, 309 (1983)

${ }^{37}$ R. F. Boivin, Line Broadening Mechanisms, PL-039, West Virginia University, Morgantown (1999)

${ }^{38}$ R. F. Boivin, Zeeman Splitting for LIF Transitions and De-convolution Technique to Extract Ion Temperatures, PL-050, West Virginia University, Morgantown (2002)

${ }^{39}$ H. Salami and A. Ross, J. Molec. Spectr. 233, 157 (2005)

40 J. C. Camparo, Contemp. Phys. 26, 443 (1985)

${ }^{41}$ C.E. Weiman and L. Holberg, Rev. Sci. Instrum. 62, 1 (1991)

${ }^{42}$ M. G. Littman and J. Metcalf, Appl. Optics 17, 2224 (1978)

${ }^{43}$ M. Born and E. Wolf, Principles of Optics, (Cambridge University Press, 1959)

${ }^{44}$ X. Sun, A Study of Ion Acceleration, Asymmetric Optical Pumping and Low Frequency Waves in Two Expanding Helicon Plasmas, Ph. D. Thesis, West Virginia University, Morgantown (2005)

${ }^{45}$ E. E. Scime, J. L. Kline, D. Mathess and C. Weber II, Rev. Sci. Instrum. 73, 1970 (2002)

${ }^{46}$ D. Leneman and W. Gekelman, Rev. Sci. Instrum. 72, 3473 (2001) 


\section{CHAPTER 4: Comparison of LIF and RFEA Methods for Measuring Ivdfs}

\subsection{Shortcomings of Conventional RFEA Data Analysis}

As discussed previously in Section 3.3.3, there are two commonly used methods of extracting IVDF information from conventional RFEA ${ }^{1}$ measurements: a) by taking the first derivative of the collector current $\left(I_{C}\right)$ with respect to the discriminating voltage and b) by plotting the parent distribution functions, corresponding to the best fit curve according to Eqn. 3.25, to the raw RFEA data. To compare the two methods in the context of extracting the IVDF, Fig. 4.1, once again shows the IVDF found by using the two methods from the same RFEA data.

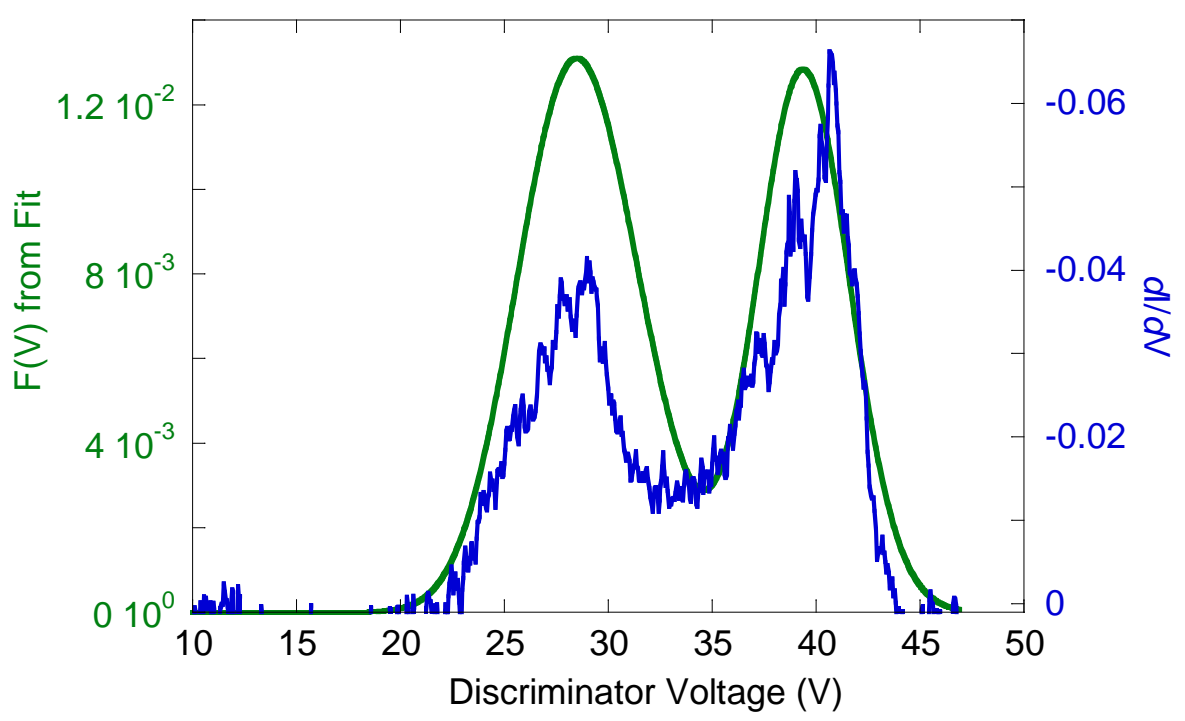

Figure 4.1. Comparing the two methods of finding IVDF from RFEA data. The green line is the parent ion population from the best fit of two drifting Maxwellian distributions. The blue line is the derivative of the collector current with respect to the discriminator voltage. The measurement location was in LEIA at the point $\mathrm{F}$ in Fig. 2.3

The most significant concern with both of these analysis approaches is that the relative ion beam population magnitude (compared to the background ion population) appears to be extremely sensitive to the analysis methodology and that these results are inconsistent with LIF measurements of the IVDF obtained in the same plasma at nearly 
the same location. Figure 4.2 shows a typical measurement of IVDF using LIF in LEIA. The measurement location was the position E in Fig 2.3. ${ }^{2,3}$ In the LIF measurement, the density of the slow background ion population is much larger than the population of the ion beam. If the measurement locations for the RFEA and LIF were the same, it is expected that the beam population as measured by LIF would be even smaller since position $\mathrm{F}$ is further downstream from the HELIX-EIA junction than position $\mathrm{E}$ in Fig 2.3. Such IVDFs are generally observed for all LIF measurements in double layer studies. $^{4,5}$ The LIF measurement also shows very clearly (from the full width at half maximum) that the background ions are relatively cold and that beam is comprised of hotter ions. Therefore, the LIF measurements disagree in both temperature and relative population density with the RFEA derived IVDF. It is worth noting that because LIF is non-invasive, it does not suffer from the sheath acceleration effects to which the RFEA is subject.

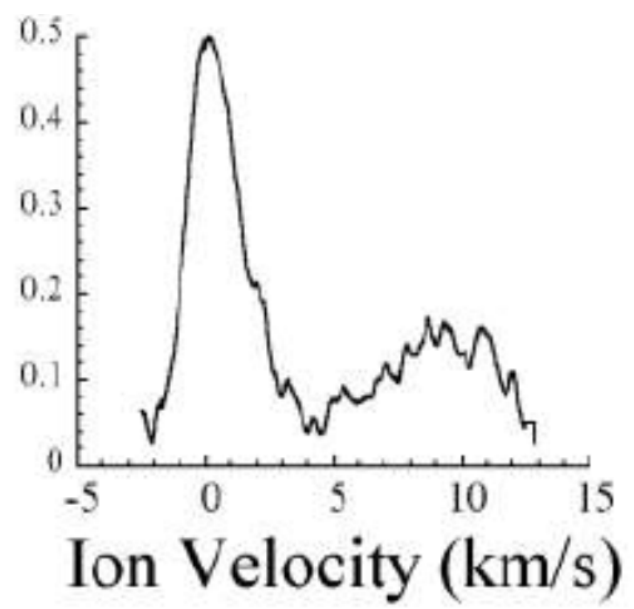

Figure 4.2. Example of a typical LIF measurement of IVDF for Ar II in LEIA (at the position E in Fig. 2.3). The figure is obtained from Ref. [3].

\subsection{An Advanced Method of RFEA Data Analysis}

Both the "derivative method" and the "beam fitting method" discussed in the previous section, ignore some key aspects of the RFEA measurement process. First, the acceleration of a drifting Maxwellian population in a spatially localized electric field (due to the sheath that forms in front of the large metallic RFEA) yields a clearly nonMaxwellian ion distribution - a result of “compression in velocity space.” Second, only 
the half of the background ion population moving towards the RFEA at the sheath boundary should make into the RFEA aperture, whereas the entire directed beam population will enter the RFEA. In the previous analysis methods, the entire background population is assumed to enter the RFEA aperture.

Therefore the effect of the acceleration of both the background and the beam ion populations through the sheath should be considered to properly analyze RFEA data and then compared to the LIF measurements of IVDF. To understand "compression in velocity space," consider two argon ions, one with a velocity of $0 \mathrm{~m} / \mathrm{s}$ (at the peak of the background population which is at rest) and the other travelling at $6926 \mathrm{~m} / \mathrm{s}(10 \mathrm{eV})$. If both experience an acceleration though a plasma sheath corresponding to $20 \mathrm{~V}$ (typical plasma potentials measured by RFEA), the final velocity of the two ions shall be 9795 $\mathrm{m} / \mathrm{s}(20 \mathrm{eV})$ and 11,997 $\mathrm{m} / \mathrm{s}(30 \mathrm{eV})$. Thus, the velocity difference between the two ions has shrunk to $\sim 2200 \mathrm{~m} / \mathrm{s}$ (from the initial $\sim 6900 \mathrm{~m} / \mathrm{s}$ ). This velocity space compression occurs because both ions gain a common energy increment, not a common velocity increment. The effect on a distribution function is to distort a Maxwellian velocity distribution into something decidedly non-Maxwellian.

To incorporate the above ideas in the analysis of RFEA data, the expression below describes the expected collector current, for two parent Maxwellian ion populations (a beam and a stationary background) that have been accelerated to the RFEA aperture by the plasma potential between the RFEA and the plasma, as a function of discriminator potential:

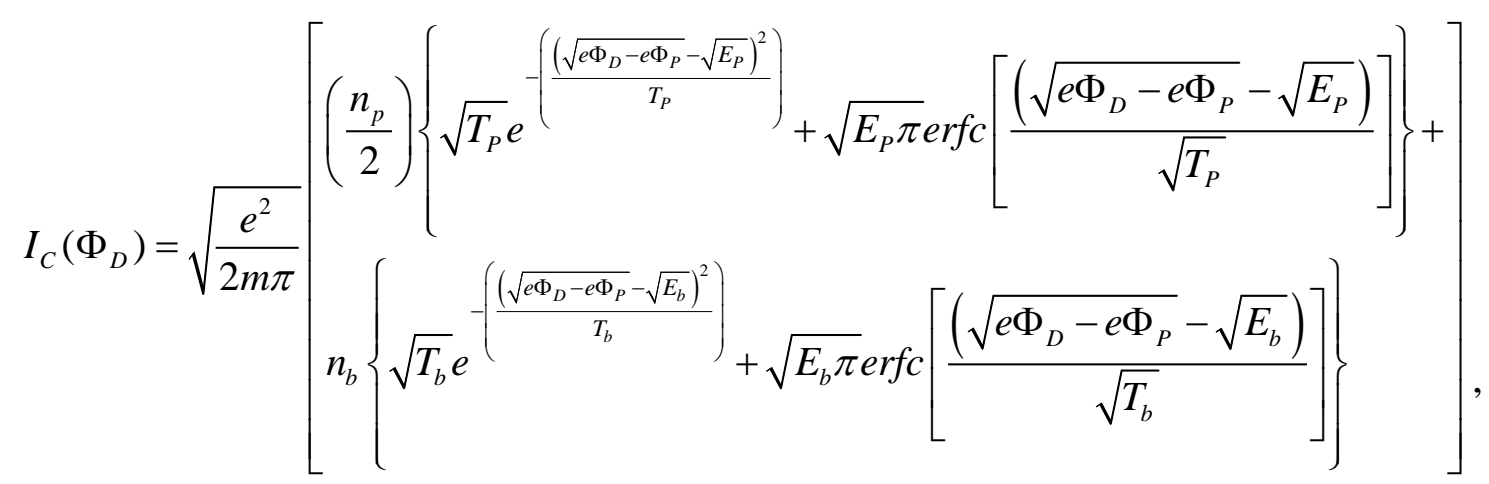


where a factor of $1 / 2$ appears in the density of the background plasma, $n_{p}$, to account for the fact that only the half of the background distribution that was traveling towards the probe is measured, whereas the entire beam population (assuming the beam was originally traveling towards the RFEA probe) is measured. ${ }^{6}$ The possibility of a drift of the background ion population in addition to the acceleration arising from the plasma potential is explicitly included in Eqn. (4.1) through the $E_{p}$ energy term. For the RFEA measurements presented in this work, the parent ion distributions are obtained by fitting Eqn. (4.1) to RFEA data.

Fig. 4.3 and Fig. 4.4 show an example of the results from such an analysis. In Fig. 4.3, the green line is raw RFEA data and the blue line gives the best fit of the expression of Eqn. 4.1 to the RFEA data. The two parent ion populations that are obtained from the fit are shown in red in Fig. 4.4.

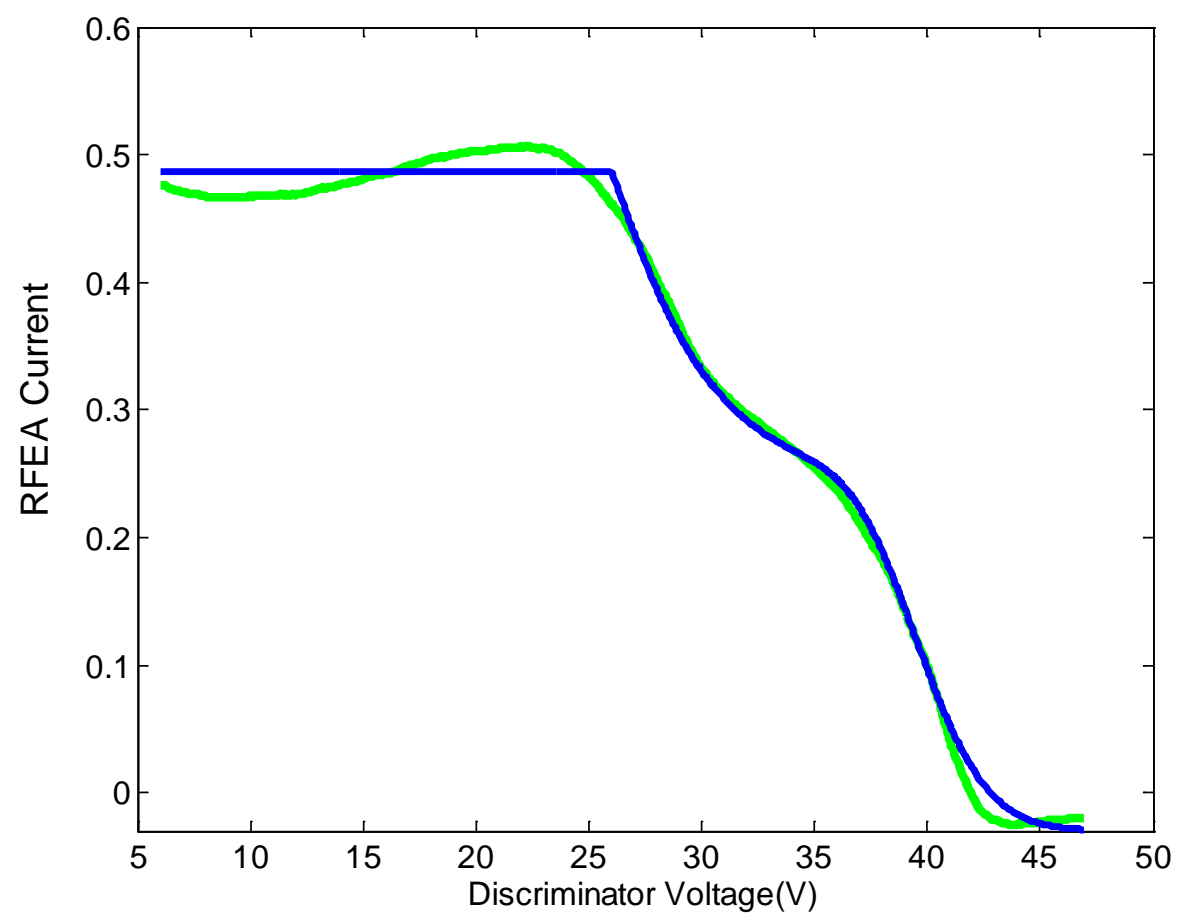

Figure 4.3. Typical RFEA measurement (green) and best fit (blue) of Eqn. 4.1 to the RFEA data. 


\subsection{IVDFs Obtained from RFEA and LIF Measurements}

When the acceleration of the background and beam ion populations through the sheath in front of the RFEA is self-consistently included by fitting the data of Fig. 4.3 with the expression given in Eqn. (4.1), the red curve shown in Fig. 4.4 is obtained for the parent ion distribution (shown in units of laser frequency offset for comparison with LIF measurements). Also shown in Fig. 4.4 is the IVDF obtained by LIF at the same location and under the same plasma conditions (green line). The beam energies obtained by both measurement techniques are in excellent agreement. ${ }^{6}$

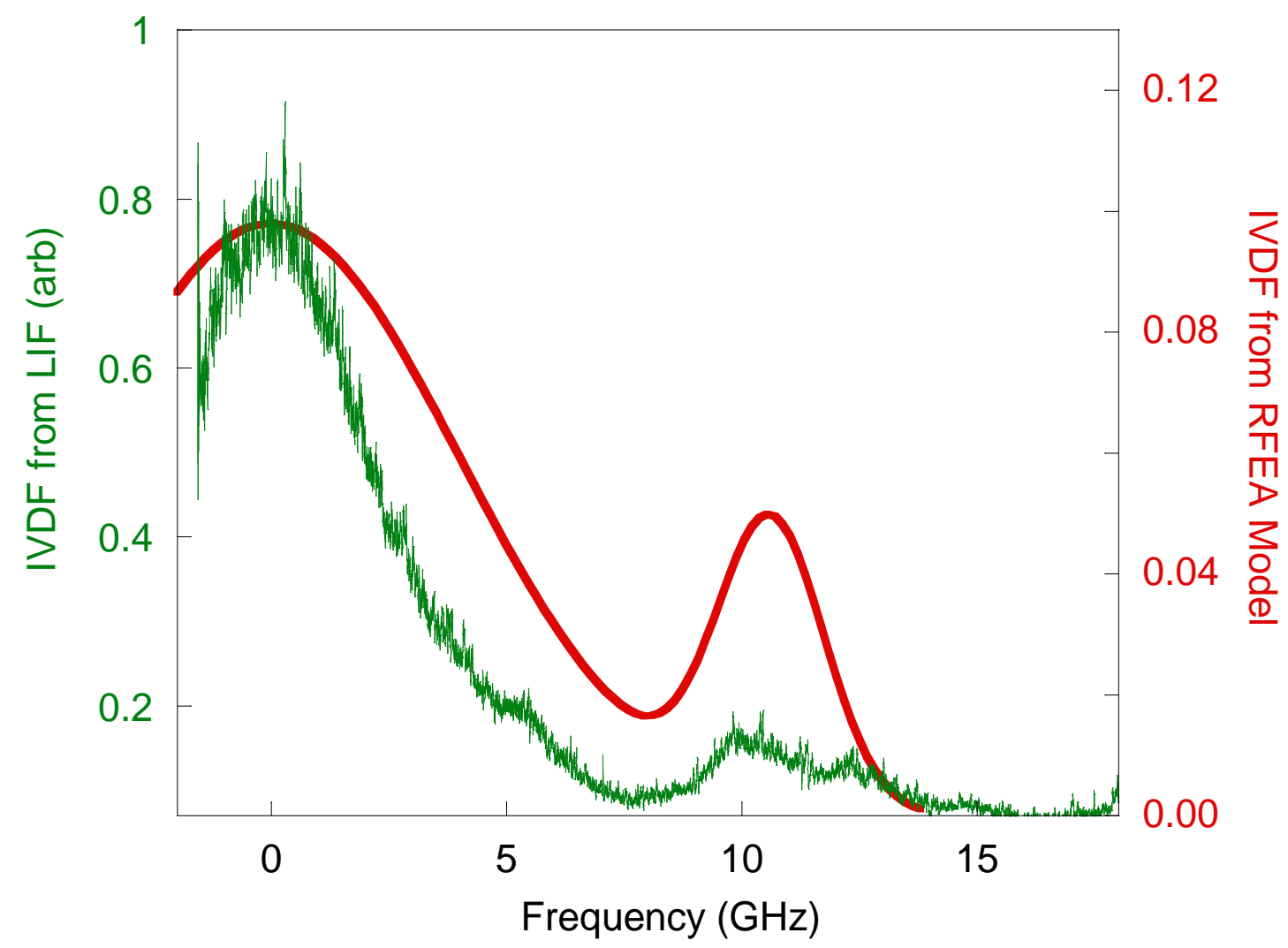

Figure 4.4. The two ion distributions obtained from fitting Eqn. 4.1 to the RFEA data (red) compared to LIF measurements (green) of the IVDF at the same axial location (position F in Fig. 2.3). The Figure is obtained from Ref. [6].

It is particularly interesting to note how this analysis method dramatically reduces the amplitude of the beam population with respect to the slower background ion plasma 
population. This is a result of correctly accounting for the greater impact of the fast ion population on the measured collector current. However, although the beam density is significantly smaller than the bulk ion density for both measurements, the beam density to bulk ion density ratio is much smaller in the LIF measurement than in the RFEA measurement. There has been a long-standing concern that LIF measurements of ion beams may underestimate the fraction of ions in a beam created upstream of the measurement location because the beam ions are no longer in the appropriate metastable state for detection by LIF (because of metastable state quenching collisions with ions, electrons, and neutrals). ${ }^{4,5}$ These results are consistent with the beam-ion metastable quenching hypothesis. Note also that the parallel ion temperature (IVDF width) is considerably larger in the RFEA measurement $(\sim 2.5 \mathrm{eV}$ ) than in the LIF measurement ( $0.9 \mathrm{eV})$. The substantially larger RFEA parallel ion temperature suggests that sloshing (back and forth ion motion) in the rf potential across the plasma sheath in front of the RFEA probe leads to an artificial broadening of an IVDF when measured with an RFEA probe in a rf plasma. Moreover, it is important to note that the LIF signal is due to the accessible metastable ions in the particular ionic state being used for the LIF scheme, while RFEA collects all the ions present in the system. Hence LIF signals are typically weaker, when compared to RFEA, since metastable quenching do not affect RFEA measurements.

Having used two different diagnostic techniques to measure the IVDF in plasma, we could compare their performance and study the intrinsic limitations in each of the methods. The RFEA is perturbative due to its large size and gets very hot in high density plasma so that it can only be used in the low density expansion chamber. Moreover, RFEA cannot distinguish between ions of different species in case of multiple ion studies. ${ }^{7}$ RFEA is also unable to measure the VDFs of neutrals. In these respects, LIF is a better choice for VDF measurements since it is non-perturbative and can be used to measure VDFs of either ions or neutral species. On the other hand LIF is a very restrictive technique. LIF requires convenient three or four state schemes ${ }^{8}$ along with an available laser with the correct wavelength for the pump transition. For LIF, the initial state should also have a large population for sufficient signal-to-noise; hence initial states are typically ground or metastable states. Thus, for many ions, neutrals, and molecules, 
LIF is not feasible. Since collisional quenching can also strongly reduce LIF signal, LIF is effective only when the three or four way schemes are available in relatively low collisionality plasmas. Finally, since LIF is typically an excited state measurement, complex models and additional measurements of plasma parameters are generally required to determine the bulk ion or neutral density from the LIF signal intensity. 


\section{References}

${ }^{1}$ C. Charles, A. W. Degeling, T. E. Sheridan, J. H. Harris, M. A. Leiberman and R. W. Boswell, Phys. Plasmas 7, 5232 (2000)

${ }^{2}$ X. Sun, A. M. Keese, C. Biloiu, E. E. Scime, A. Meige, C. Charles and R. W. Boswell, Phys. Rev. Lett. 95, 025004, (2005)

${ }^{3}$ X. Sun, A Study of Ion Acceleration, Asymmetric Optical Pumping and Low Frequency Waves in Two Expanding Helicon Plasmas, Ph. D. Thesis, West Virginia University, Morgantown (2005)

${ }^{4}$ S. A. Cohen, N. S. Seifert, S. Stange, E. E. Scime, R. F. Boivin and F. Levinton, Phys. Plasmas 10, 2593 (2003)

${ }^{5}$ A.M. Keesee, E. E. Scime, C. Charles, A. Meige and R. W. Boswell Phys. Plasmas 12, 093502 (2005)

${ }^{6}$ Z. Harvey, S. Chakraborty Thakur, A. Hansen, R. A. Hardin, W. S. Przybysz and E. E. Scime, Rev. Sci. Instrum. 79, 10F314 (2008)

${ }^{7}$ I. A. Biloiu, Laser Induced Fluorescence Studies of Ion Acceleration in Single and Multiple Species Expanding Plasmas, Ph. D. Thesis, West Virginia University, Morgantown (2009)

${ }^{8}$ A. M. Keesee, R. Boivin and E. E. Scime, Rev. Sci. Instrum. 75, 4091 (2004) 


\section{Chapter 5: Studies of Electrostatic Double Layers}

Mutually consistent measurements of ion beam energy and density obtained through retarding field energy analyzer and laser induced fluorescence IVDF measurements indicate that a stable ion beam of approximately $15 \mathrm{eV}\left(\sim 3 k_{B} T_{e}\right)$ appears in an expanding, low pressure, argon helicon plasma for source antenna frequencies above 11.5 MHz. The ion beam is evidence of the formation of an electrostatic double layer upstream of the measurement location. At lower antenna frequencies, large electrostatic instabilities appear downstream of the expansion region and a well-formed ion beam is not observed. Further studies of the low frequency $(\sim 17.5 \mathrm{kHz})$ electrostatic fluctuations suggest that they arise from beam-driven, ion acoustic instabilities in the double layer. We also observe a sharp increase in the upstream density at the same threshold frequency; confirming a theoretical model that predicts an increase in the upstream density due to enhanced ionization resulting from electrons accelerated upstream by the double layer.

\subsection{Review of Double Layers}

In the simplest form, a Double Layer (DL) can be thought of as two spatially separated layers of charge, one positive and the other negative. In other words, a DL is similar to a freestanding (or moving) sheath that can appear anywhere in a plasma far away from the boundary walls (in case of laboratory plasma). The physical location of the DL distinguishes it from a conventional sheath, which appears at the surface of an object inserted into the plasma or at the plasma boundary. ${ }^{1}$ Essentially, DLs are narrow, local regions of strong electric potential gradient isolated from plasma boundaries. A schematic of a typical DL is shown in Fig. 5.1. Note that even in quasi-neutral plasma, quasi-neutrality is violated within the DL. DL thicknesses are predicted to be $10-50$ times the Debye length. ${ }^{2}$ DLs often separate regions of plasma with widely different densities and temperatures and are an important mechanism for the acceleration of charged particles along magnetic fields in astrophysical and laboratory plasmas. A very nice review of some early studies of DLs is given by Block. ${ }^{2}$ 

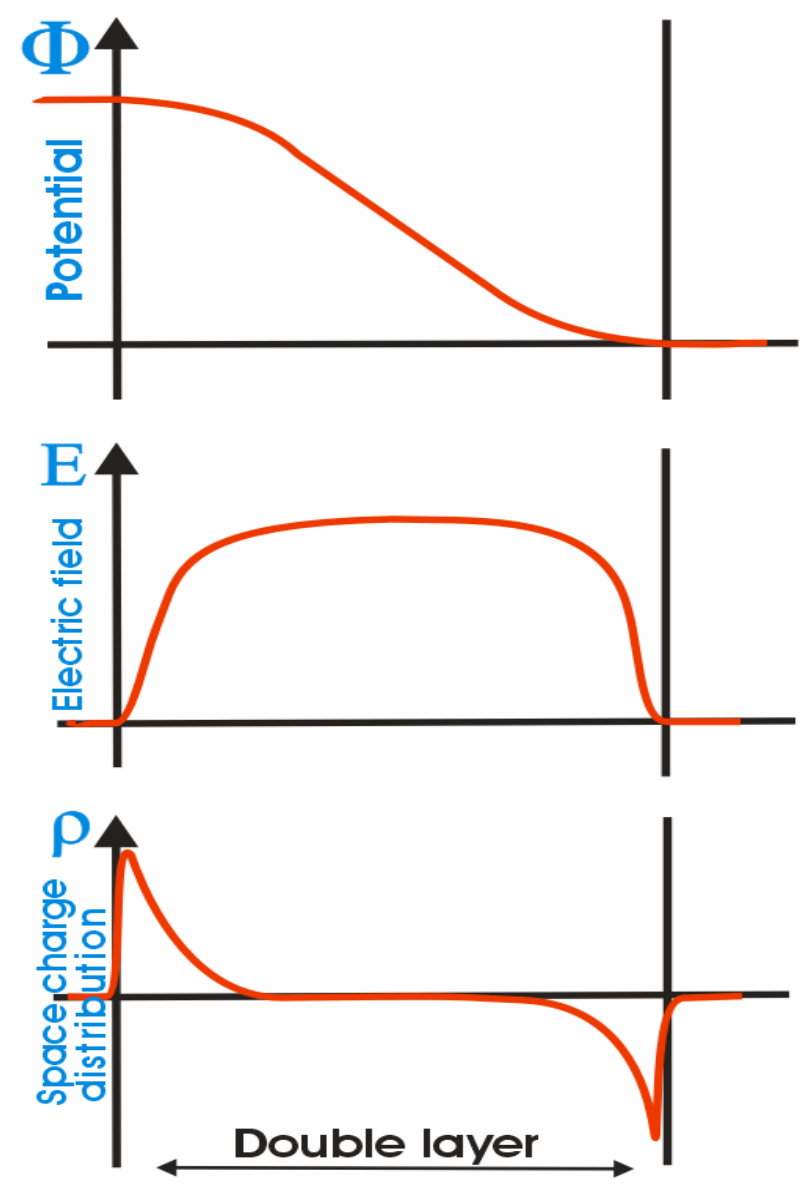

Figure 5.1. A cartoon showing the characteristics of a double layer. Figure from Ref. [2].

Hannes Alfvén suggested that a possible mechanism for the formation of the aurora is the precipitation of energetic electrons that have been accelerated by electric $\mathrm{DLs}^{3}$ in space into the upper atmosphere. Later observations ${ }^{4}$ provided strong evidence supporting Alfven's hypothesis. Since then DLs have been invoked in the discussions of physical phenomena as diverse as solar flares, ${ }^{5}$ high power gas lasers ${ }^{6}$, laser ablated plasmas $^{7}$ and helicon plasma thrusters. ${ }^{8}$ Previous observations of DLs in space, ${ }^{4}$ in laboratory experiments ${ }^{9,10,11,12,13}$ and simulations. ${ }^{14,15}$ have occurred in systems driven by electric current, externally imposed potential differences, or the merging of two plasmas with different initial electron temperatures.

It was first predicted in 1981 by Perkins ${ }^{16}$ in an analytical study that a DL can be formed in a current-free plasma expanding in a divergent magnetic field. In spite of 
experimental observations of ion acceleration in expanding current free plasma, ${ }^{17,18,19}$ there was no clear evidence of DL formation in those early experiments. On the other hand, unambiguous observations of DL formation in current-driven plasmas expanding in a diverging magnetic field, suggested that the divergent magnetic fields themselves could play an important role in DL formation. ${ }^{20}$

Current-free plasma expansion in a diverging magnetic field is surprisingly common in nature in a wide variety of spatial scales. Plasma expansion is essentially equivalent to a pressure gradient arising from change in the plasma density. This density gradient can give rise to a potential gradient which in turn retards the lighter electrons but accelerates the heavier ions downstream. A classic example of this process is the formation of the interplanetary electric field due to solar wind expansion. Under isothermal, collisional conditions, the electron density depends on the plasma potential through the classic Boltzmann equation. However, in collision-less plasmas, the mean free path for ion collisions (charge exchange and elastic collisions, for example) can be much longer than both the length scale of the magnetic field gradient or the plasma expansion. Electrostatic DLs can form under such circumstances. A typical DL potential structure with its associated charged particle populations is shown in Fig. 5.2. To maintain a DL, at least three of the four particle populations: free (or accelerated) and trapped (or retarded) ions and electrons, must be present.

Since freely expanding electrons can create their own ambipolar electric field that accelerates out of the source at the sound speed, a potential drop larger than $k_{B} T_{e}$ is an often cited requirement for a plasma structure to be identified as a DL. Weak double layers are those where the potential drop is a few times $k_{B} T_{e}{ }^{21}$ Strong DLs can have potential drops of many times the electron temperature: many hundreds, if not thousands, times the electron thermal energy. ${ }^{22,23,24,25}$ However, a potential drop with less than $k_{B} T_{e}$ is possible for DLs that are of the slow ion acoustic soliton type. In the literature it is also commonly stated that the thickness of a DL should be smaller than the ion mean-free path. ${ }^{2}$ But in laboratory plasmas, a DL's thickness can vary from several ${ }^{26}$ to thousands of Debye lengths. ${ }^{23}$ Experimental studies of DLs have also been performed in plasmas with widely varying plasma densities ${ }^{27}$; ranging from $10^{6}$ to $10^{20} \mathrm{~cm}^{-3}$. Both one and two dimensional $\mathrm{DLs}^{28}$ and also stationary and moving $\mathrm{DLs}^{29}$ have been experimentally 
studied. DLs have also been studied in freely expanding laboratory plasmas if the plasma source contains both Maxwellian and energetic electrons. ${ }^{30,31,32}$ The freely expanding experiments suggested that the DL was generated self-consistently to maintain the separation of the distinct electron populations.

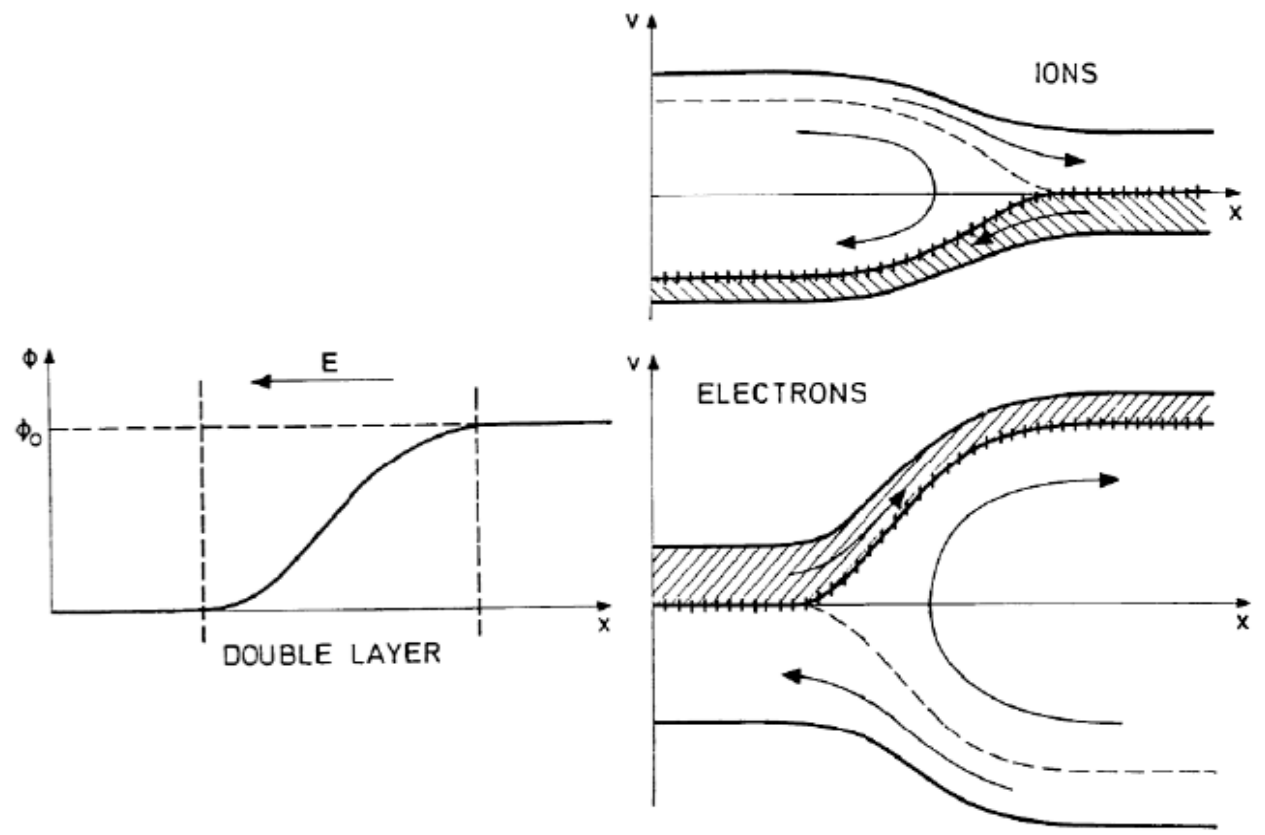

Figure 5.2. Potential distribution $\Phi(x)$, and the ion and electron phase space distributions across a DL with potential drop $\Phi_{0}$ (and the corresponding internal electric field $\mathbf{E}$ ). The hatched areas indicate accelerated populations. The reflection of ions and electrons within the separatrices (symmetrically placed broken and barred curves) are indicated by the arrows symbolizing velocity reversal. Ions or electrons with energy larger than $\Phi_{0}$ (with very large initial velocity) are not reflected but can pass through the DL, as indicated.

\subsection{Double Layers in Helicon Plasmas}

Helicon source plasma is a high-density, current-free, rf-wave driven plasma. Helicon sources can be operated either in a steady-state or pulsed mode. An often cited characteristic of helicon plasma is the appearance of a "bright core" (helicon mode) ${ }^{33}$ as the source transitions from the relatively low density "C" and "L" modes (Capacitive and Inductive modes respectively) into the high density "Helicon mode." The transition is typically initiated by increase in the magnetic field strength or power fed to the antenna. ${ }^{34}$ 
Although the mechanism(s) responsible for the highly efficient rf coupling in helicon sources is a subject of ongoing research (see Section 2.2), their high efficiency, compatibility with low mass ions for high specific impulse operation, steady-state operation without internal electrodes, and modest magnetic field strengths make helicon plasma sources ideal candidates for thermal plasma propulsion systems. In a typical thermal plasma thruster, the plasma is heated and the random energy of the hot propellant converted into directed flow in a physical or magnetic nozzle. ${ }^{35,36,37}$

In 2003, working with the Chi-Kung ${ }^{38}$ helicon plasma source in Australia National University (ANU), Charles and Boswell ${ }^{39}$ reported measurements of a discontinuity in the plasma potential, a typical signature of an electric double layer, at the end of a low pressure $(\leqslant 0.5$ mTorr) argon helicon plasma. Using a retarding field energy analyzer, ${ }^{40}$ they mapped the plasma potential along the axis of the device and found that when the neutral pressure dropped below 0.5 mTorr, a "rapid and discontinuous change in the plasma potential close to the exit of the source" appeared (see Fig. 5.2). In 2004, RFEA measurements in the same experimental device confirmed that ions are accelerated through the "potential drop" structure in both argon ${ }^{41}$ and hydrogen ${ }^{42}$ plasmas. The combination of a strong gradient in the plasma potential and the observation of an ion beam in the diffusion region of the Chi-Kung helicon plasma expanding in a diverging magnetic field demonstrated that a DL was being created in the expanding helicon plasma.

Almost simultaneously Cohen et al. ${ }^{43}$ at Princeton Plasma Physics Laboratory (PPPL) and Sun et al. ${ }^{44}$ in West Virginia University (WVU) reported measurements of supersonic ion flows in their expanding helicon plasma experiments: MNX (Magnetic Nozzle Experiment) and HELIX-LEIA, respectively. The ion flow measurements were accomplished with the same portable, tunable diode laser based LIF diagnostic. ${ }^{45}$ All the three helicon source experiments have similar magnetic field expansion regions, the same signatures of a sharp potential gradient near the expansion region, and observations of a supersonic ion beam in the diffusion region; signatures of a DL in the expanding helicon plasma. $^{46,43}$ 


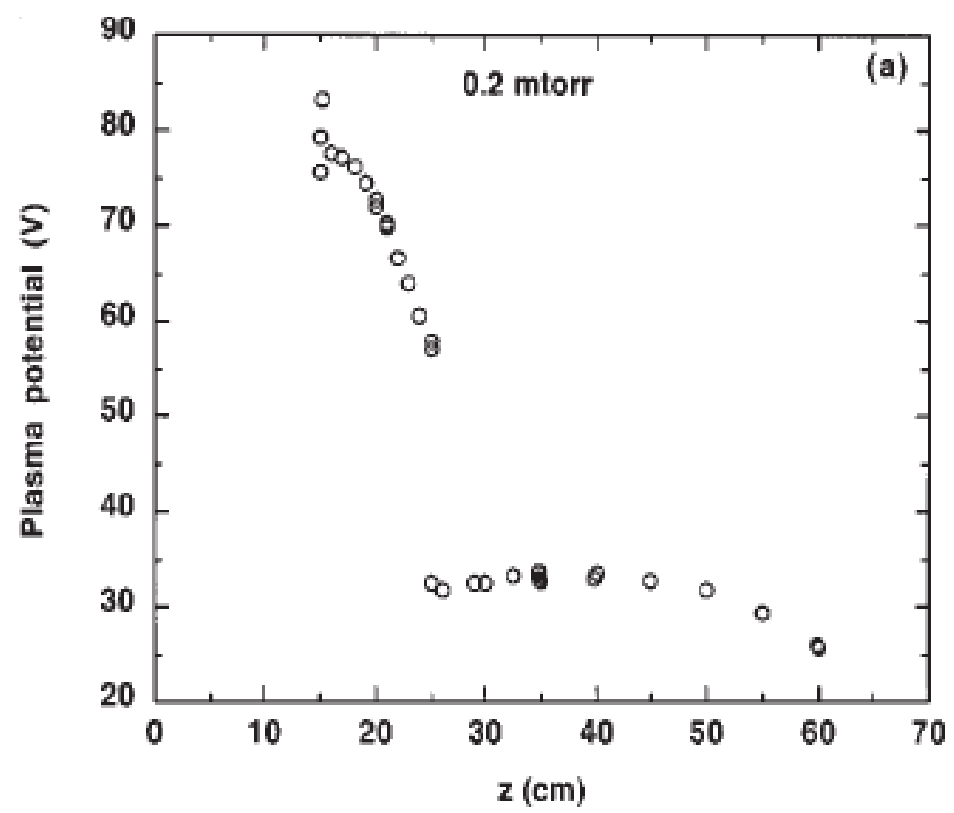

Figure 5.3. Measured plasma potential (using a retarding field energy analyzer) along the z-axis of the Chi-Kung helicon plasma source. Figure adapted from Ref. [39].

A one-dimensional, unmagnetized, MCC-PIC (Monte Carlo Collisions - Particle in Cell code) hybrid simulation (particle ions and fluid electrons) that modeled plasma expansion in a diverging magnetic field with a position dependant particle loss rate provided further evidence that a DL can form in current-free expanding plasma. ${ }^{47}$ In that simulation, the DL formed at the location of rapid plasma expansion. The DL width was a few tens of Debye lengths. A low energy population of ions, created by ionization and by charge-exchange collisions was observed. In addition to this ion population, downstream (in the diffusion region) of the DL a high-energy ion population ( $v_{\text {beam }} \sim$ $2 v_{\text {sound }}$ ) accelerated through the DL was observed.

The roughly $14 \mathrm{eV}$ potential drop through the DL was obtained for an argon plasma at a pressure of 0.5 mTorr, an electron density of $6.5 \times 10^{8} \mathrm{~cm}^{-3}$ and an electron temperature of $7.2 \mathrm{eV}$ (so $\Phi \sim 2 k_{B} T_{e}$ ). The total ion acceleration occurred over roughly an ion mean-free path. The predictions from the hybrid simulation were consistent with the experimental results from all the three devices: Chi-Kung, MNX and HELIX-LEIA. 


\subsection{Theory of Formation of Current-Free Double Layers in Expanding Helicon Plasmas}

Even though there are theoretical studies of DLs since the 1970s, ${ }^{2}$ most have dealt with current-driven DLs. ${ }^{48}$ Reference [49] includes a nice description of the details of the theory of one dimensional DL formation in collisionless plasma. The necessary conditions to embed a DL in a quasi-neutral plasma include the existence of four groups of charged particles inside the DL: (a) thermal ions, (b) accelerated ions flowing downstream, (c) accelerated electrons flowing upstream and (d) thermal electrons. ${ }^{50}$ For assumed mono-energetic accelerated species and Maxwellian thermal species, the density ratios of the species at the edge of the DL and for a given DL potential drop, the entering velocities necessary for the accelerated species are calculable.

More recently, theoretical models have been developed for current-free DLs expanding magnetic fields in laboratory plasmas ${ }^{51,52,53}$ in the solar corona [53] and at the boundary between the ionosphere and the auroral cavity [53]. In References [51] and [52], a diffusion-controlled model describes the formation of a current-free DL in a

plasma expanding from a small-diameter, dielectric source chamber (upstream) to a large-diameter, conducting expansion chamber (downstream); similar to our experimental configuration and the configuration of a variety of plasma sources used in the plasma processing industry and in other laboratory experiments. Here upstream and downstream are defined with respect to the spatial location of the DL.

The diffusion-controlled model couples the dynamics of the particles in the nonneutral DL to the diffusive flows of the quasi-neutral plasma in the source and expansion chambers. To ensure that the DL is current-free, in addition to the conventional four charge populations, the model requires another group of counter-streaming electron population, formed by the reflection of almost all of the accelerated electrons from the sheath at the insulated end wall of the source chamber. The potential difference across the DL is determined by the upstream and downstream particle balances. Since the upstream radius is smaller than the downstream radius, ionization by thermal electrons upstream is not enough to balance the larger particle losses upstream. An additional source of upstream ionization becomes necessary and is provided by the accelerated group of electrons. In this model, the DL vanishes at very low pressures as the maximum 
ionization rate for the accelerated electrons upstream is insufficient to balance the excess upstream particle loss. At very high pressures, the system length becomes comparable to the energy relaxation length for ionizing electrons. Since electrons are heated upstream, the downstream ionizing electron density decreases at higher pressures. When the downstream and upstream ionization rates become equal to the corresponding particle loss rates, the additional ionization provided by the accelerated electrons is unnecessary and the DL vanishes. The model successfully explains the experimental observations of both the lower and upper thresholds on pressure for the formation of DLs.

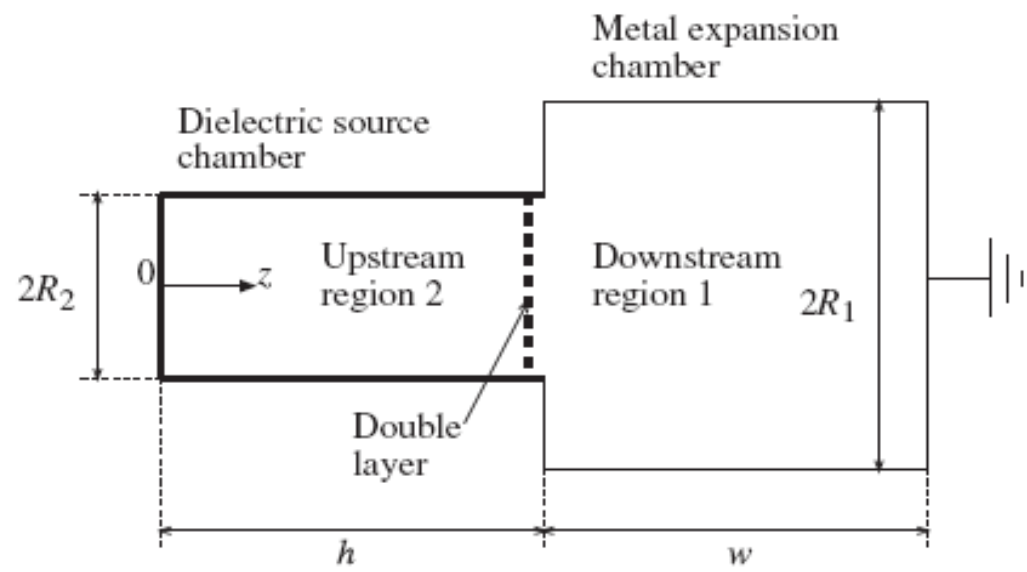

Figure 5.4. Schematic diagram of DL formation at the junction between a smaller diameter “Upstream” plasma source and a larger diameter "Downstream” expansion chamber in Chi-Kung. The values of the shown parameters are: $h=31 \mathrm{~cm} ; R_{2}=6.85 \mathrm{~cm} ; w=29.4 \mathrm{~cm} ; R_{1}=15.9 \mathrm{~cm}$. Figure is obtained from Ref. [51].

In the following calculation of densities of the different populations that lead to the formation of a DL in an expanding helicon plasma, following the procedure and notation of Reference [50], the subscripts $a, b, c$ and $d$ refer to thermal ions, accelerated ions, accelerated electrons and thermal electrons, respectively. The subscripts 1 and 2 refer to downstream and upstream DL edges. $M$ and $m$ stand for the ion and electron masses, respectively. The potential is chosen to be zero at the downstream edge and Vs at the upstream edge. The charge density within the DL is calculated by solving Poisson's 
equation for the potential, $V$. For the thermal ions and electrons, the charge densities are given by the Boltzmann relations

$$
\begin{gathered}
\rho_{a}=\rho_{a 1} e^{-V / T_{i}}, \\
\rho_{d}=\rho_{d 2} e^{-\left(V_{s}-V\right) / T_{e}},
\end{gathered}
$$

where $\rho_{a_{1}}$ and $\rho_{d_{2}}$ are the thermal ion and thermal electron densities at the downstream and upstream edges, respectively. Here $T_{i}\left(=k_{B} T_{i} / e\right)$ and $T_{e}\left(=k_{B} T_{e} / e\right)$ represent the ion temperature and the electron temperature in the equivalent voltage units (volts).

For mono energetic ions entering the DL upstream with velocity $v_{i}$ and charge density $\rho_{b 2}$, the charge density is determined by the particle flux and energy conservation within the DL:

$$
\rho_{b} v_{z}=\rho_{b 2} v_{i}
$$

where

$$
v_{z}^{2}=v_{i}^{2}+2 e\left(V_{s}-V\right) / M .
$$

Combining terms, we get

$$
\rho_{b}=\rho_{b 2}\left[1+2 e(V s-V) / M v_{i}^{2}\right]^{-1 / 2} .
$$

These calculations follow the procedure outlined in Reference [50], but an initially nearhalf-Maxwellian $\left[f_{c 1}\right]$ distribution of accelerated electrons, instead of mono-energetic ones, is introduced to make the model more realistically describe the laboratory experiments. Since, at the very low experimental pressures ( $<0.5$ mTorr), the energy relaxation length for electrons with energies below the first excitation energies of argon 
(11.6 $\mathrm{eV})$ is much larger than the system length, the characteristic electron temperatures $\left(T_{e}\right.$ ) for both the accelerated electron population $(c)$ and the trapped electrons are assumed to be the same. The velocity $v_{e}$ is on order of $\left(e T_{i} / m\right)^{1 / 2}$, which is much smaller than the electron thermal velocity. Moreover, the model also requires the distribution function to be zero at $v_{z}=0$.

So for $v_{z}>v_{e}$, the near-half-Maxwellian distribution is given by

$$
f_{c 1}=\frac{2 n_{c 1}}{\operatorname{erfc}\left(\zeta_{1}\right)}\left(\frac{m}{2 \pi e T_{e}}\right)^{1 / 2} e^{-m v_{2}^{2} / 2 e T_{e}}
$$

and for $v_{z}<v_{e}$,

$$
f_{c 1}=0
$$

where $\zeta_{1}=\left(m v_{e}^{2} / 2 e T_{e}\right)^{1 / 2}, n_{c 1}=-\rho_{c 1} / e, e r f c$ is the complimentary error function and the normalization is given by $\int f_{c 1} d v_{z}=n_{c 1}$. The corresponding charge density is

$$
\rho_{c}=-e \int_{v_{e}}^{\infty} v_{z}\left(v_{z}^{2}+2 e V / m\right)^{-1 / 2} f_{c 1} d v_{z} .
$$

Upon evaluating the integral, we get

$$
\rho_{c}=\rho_{c i} \frac{\operatorname{erfc}(\zeta)}{\operatorname{erfc}\left(\zeta_{1}\right)} e^{-V / T_{e}}
$$

where $\zeta=\left[\left(m v_{e}^{2}+2 e V\right) / 2 e T_{e}\right]^{1 / 2}$ 
Defining some normalized quantities:

$$
\begin{gathered}
\tau_{e}=T_{e} / V_{s} \text { and } \tau_{i}=T_{i} / V_{s} \\
\Phi_{e}=m v_{e}^{2} / 2 e V_{s}, \Phi_{i}=M v_{i}^{2} / 2 e V_{s}, \\
\eta=V / V_{s},
\end{gathered}
$$

and

$$
\zeta_{1}=\left(\Phi_{e} / \tau_{e}\right)^{1 / 2} \text { and } \zeta_{1}=\left[(\Phi e+\eta) / \tau_{e}\right]^{1 / 2}
$$

The total charge density is

$$
\rho(\eta)=\rho_{a}+\rho_{b}+\rho_{c}+\rho_{d}
$$

And the boundary conditions at the upstream and downstream edges of the current-free DL are:

$$
\begin{gathered}
\rho=0 \text { at } \eta=0 \quad \text { and } \quad \rho=0 \text { at } \eta=1 \\
\frac{d \rho}{d \eta}=0 \text { at } \eta=0 \quad \text { and } \quad \frac{d \rho}{d \eta}=0 \text { at } \eta=1
\end{gathered}
$$

The boundary conditions in Eqn. (5.16) are equivalent to the Bohm condition ${ }^{54}$ for a collisionless sheath in contact with collisionless plasma; the derivatives of the total electron and ion densities at the sheath edge are equal.

The four boundary conditions in Eqns. (5.15) and (5.16) can be written in the form of a 4 x 4 matrix equation. To obtain a non-trivial solution, the determinant of the coefficient matrix should vanish. Another condition used to determine the parameters of 
the DL is that the total charge (sum of the positive and negative charges) in the DL should vanish, or equivalently, the total force acting on the DL must vanish. This yields

$$
\int_{0}^{1} \rho(\eta) d \eta=0
$$

Eq. 5.17 is the constraint that a zero electric field at one edge of the DL forces the appearance of a similarly vanishing electric field at the opposite edge of the DL. Evaluating Eqn. (5.17),

$$
E_{1} \rho_{a 1}+E_{2} \rho_{b 2}+E_{3} \rho_{c 1}+E_{4} \rho_{d 2}=0
$$

Where:

$$
\begin{aligned}
& E_{1}=\tau_{i}\left(1-e^{-1 / \tau_{i}}\right) ; \\
& E_{2}=2 \Phi_{i}\left[\left(1+\Phi_{i}^{-1}\right)^{1 / 2}-1\right] ; \\
& E_{3}=\tau_{e}\left[2\left(\zeta_{2}-\zeta_{1}\right) e^{-\zeta_{1}^{2}} / \sqrt{\pi} \operatorname{erfc}\left(\zeta_{1}\right)+e^{1 / \tau_{e}} \operatorname{erfc}\left(\zeta_{2}\right) / \operatorname{erfc}\left(\zeta_{1}\right)-1\right] ; \\
& E_{4}=\tau e\left(1-e^{-1 / \tau_{e}}\right) .
\end{aligned}
$$

For given values of $\tau_{i}$ and $\tau_{e}$, the simultaneous solutions of Eqns. (5.15) to (5.18) determine $\Phi_{i}$ and $\Phi_{e}$. Once these are calculated, the densities can be found from the ratios of the individual matrix elements corresponding to the Eqns. (5.15) and (5.16). The Eqns. (5.15) to (5.18) are highly non-linear and have to be solved numerically. The solutions have been obtained and verified for their relative accuracies to within $0.1 \%$. Solving the equations consistently, the density ratios of the four particle groups can be found initially at the downstream and upstream edges of a DL, for given values of $T_{i} / T_{e}$ and $V_{s} / T_{e}$. 

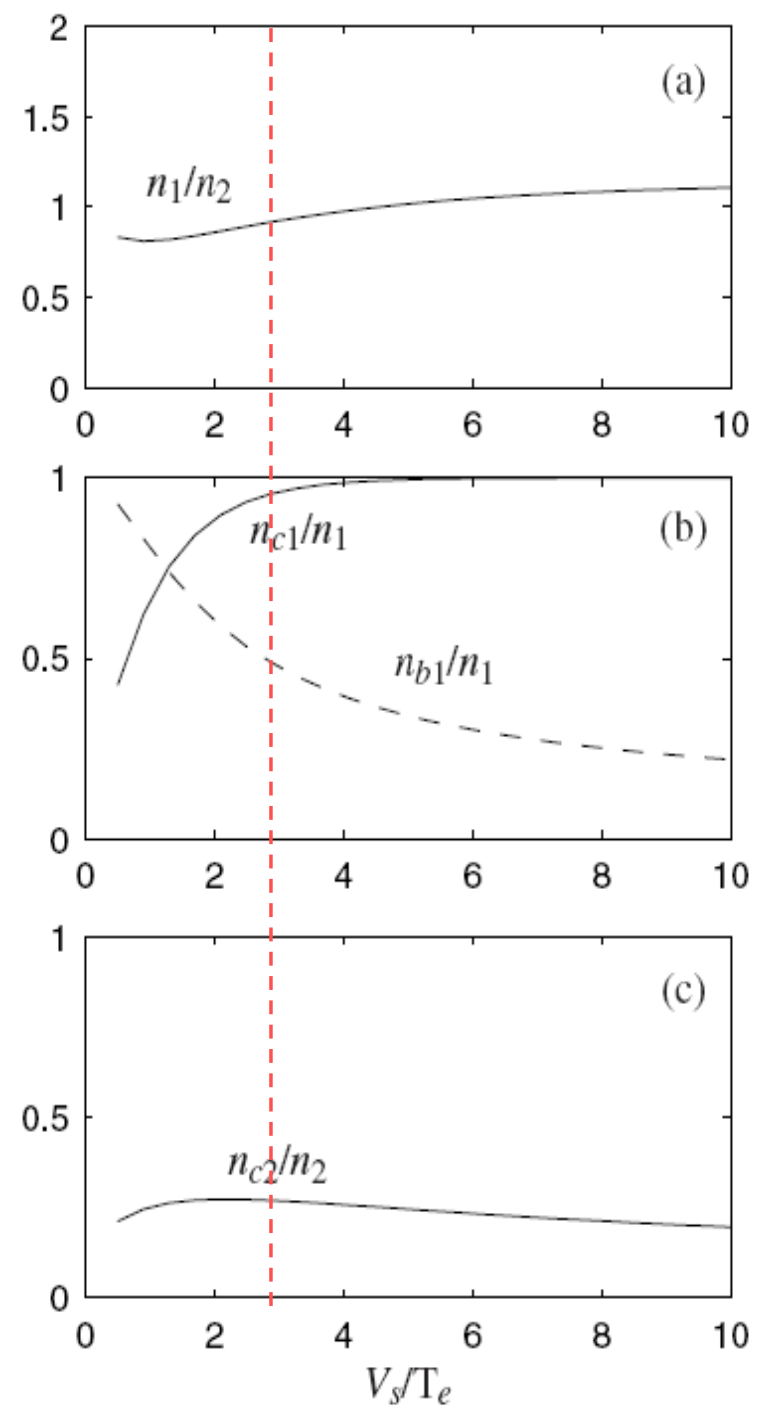

Figure 5.5. Predicted density ratios versus DL strength ( $V_{s} / T_{e}$ ); (a) $n_{1} / n_{2}$; (b) $n_{c 1} / n_{1}$ (solid) and $n_{b 1} / n_{1}$ (dashed) and (c) $n_{c 2} / n_{2}$. The figure is adapted from Ref. [51]

Fig. (5.5) shows the density ratios as a function of the DL strength $\left(V_{s} / T_{e}\right)$ for an assumed $T_{i} / T_{e}$ ratio of 0.05 (for comparison, in our experiments $T_{i} / T_{e} \sim 0.06-0.1$ ). In our experiments, the strength of the DL is approximately $V_{s} / T_{e}=3$, which is marked with a red dashed line in Fig. 5.5. The ratio of the total downstream to upstream density $\left(n_{1} / n_{2}\right.$; where $n_{1}=n_{a 1}+n_{b 1}$ and $\left.n_{2}=n_{a 2}+n_{b 2}\right)$ is nearly constant at unity as a function of the DL strength. Fig. (5.5b) shows the densities for the accelerated electron group (solid line) and the accelerated ion group (dashed line) with respect to the downstream density. Note that for our DL strength of $V_{s} / T_{e}=3$, the density of the group of electrons accelerated 
from downstream to upstream is about $90 \%$ of the total downstream density. Fig. (5.5c) shows the ratio of the accelerated electrons with respect to the total upstream density. The accelerated electrons comprise about $20 \%$ of the total upstream density almost independent of the DL strength. The key point of these numerical results is the prediction of the presence of a significant accelerated electron population upstream of the DL.

Since the source chamber is insulating in our experiments, there should be no net electrical current flowing into the source. To apply the above results to our experimental conditions, a fifth species is introduced, which is formed by the reflection of almost the entire accelerated electron group by the sheath formed at the upstream wall (which defines the end of the source chamber). It is found that this reflected electron group contributes the same charge density to the DL and to the downstream plasma as the original electron group. Hence the DL solution remains unchanged by the addition of the group. In that sense, there is no fundamental distinction between a current free and a current-carrying DL. ${ }^{55}$ A slight imbalance in the currents between the incident and reflected accelerated and thermal electron groups balances the flow of ions to the upstream wall which results in a floating potential that forms across the sheath at the upstream wall.

To calculate the electron temperature $\left(T_{e}\right)$ in the entire system at any given pressure $(p)$ and neutral gas density $\left(n_{g}\right)$, the downstream particle balance is determined by low pressure diffusion theory for unmagnetized plasma, ${ }^{56}$

$$
K_{i z} n_{g} \pi R_{1}^{2} \cdot 2 w=\left(2 \pi R_{1}^{2} h_{2 w}+2 \pi R_{1} \cdot 2 w h_{R_{1}}\right) u_{B}
$$

where $K_{i z}\left(T_{e}\right)$ is the ionization rate coefficient for thermal electrons; $h_{2 w}=0.86\left(3+w / \lambda_{i}\right)^{-1 / 2}$ and $h_{R_{1}}=0.8\left(4+R_{1} / \lambda_{i}\right)^{-1 / 2}$ are the axial and radial edge-to-center density ratios as given by low pressure diffusion theory. ${ }^{56} \lambda_{i} \approx(330 p)^{-1}$ is the ion mean free path in argon, where $\lambda_{i}$ is in $\mathrm{cm}$ and $p$ is in Torr. It has been assumed that the flux, due to the thermal ion population moving upstream into the source chamber is negligible (which is the typical behavior in helicon experiments). 
The DL potential drop $\left(V_{s}\right)$ is then determined by the upstream particle balance, which is derived from a simple one-dimensional diffusion model. Since the upstream radius (of the source chamber) is much smaller than the downstream radius (expansion chamber), ionization by thermal electrons upstream is insufficient to balance the larger upstream particles losses at low pressure. An additional source of upstream ionization is required and it is provided by the electrons being accelerated upstream through the DL. Let $n_{i}, n_{c 2}$ and $n_{d}=n_{i}-n_{c 2}$ be the densities of the ions, accelerated electrons and thermal electron respectively. The upstream diffusion equation is then given by:

$$
-D_{A 2} \frac{d^{2} n_{i}}{d z^{2}}+v_{R} n_{i}=n_{g}\left(K_{i z} n_{d 2}+K_{i z c} n_{c 2}\right)
$$

where

$D_{A 2}=h_{R 2} u_{B} R_{2}$ is the low pressure ambipolar diffusion coefficient;

$v_{R}=2 h_{R 2} u_{B} / R_{2}$ is the radial loss frequency;

and $K_{i z c}$ is the ionization rate coefficient for the accelerated electron group (a function of both $V_{s}$ and $T_{e}$ ).

Assuming a symmetric solution for the ion density about $z=h / 2$;

$$
n_{i}=\beta n_{c 2}-n_{i 0} \cosh \gamma(z-h / 2)
$$

where

$$
\beta=\frac{n_{g}\left(K_{i z c}-K_{i z}\right)}{\left(v_{R}-K_{i z} n_{g}\right)}
$$

and

$$
\gamma^{2}=\frac{\left(v_{R}-K_{i z} n_{g}\right)}{D_{A 2}}
$$


is the square of the axial decay constant. Setting the ion flux $\left(\Gamma_{i}=-D_{A 2}(d n / d z)\right)$ to $n_{i} v_{i}$ at $z=h$, determines $n_{i 0}$, where $v_{i}$ is the velocity of the mono-energetic ions entering the DL upstream. The complete solution is then given by:

$$
n_{i}=\left[1-\frac{\cosh \gamma(z-h / 2)}{\frac{\gamma D_{A 2}}{v i} \sinh \frac{\gamma h}{2}+\cosh \frac{\gamma h}{2}}\right] \beta n_{c 2}
$$

Evaluating this equation at the upstream edge of the DL, gives:

$$
\beta=\left(1+\frac{v_{i}}{\gamma D_{A 2}} \operatorname{coth} \frac{\gamma h}{2}\right) \frac{n_{i 2}}{n_{c 2}}
$$

where $n_{i 2}=n_{a 2}+n_{b 2}$ is the total ion density at the upstream edge. The ratio $n_{i 2} / n_{c 2}$ is evaluated from the simultaneous solutions of Eqns. (5.15) to (5.18). Finally, equating Eqn. (5.22) to Eqn. (5.25) yields

$$
\frac{n_{g}\left(K_{i z c}-K_{i z}\right)}{\left(v_{R}-K_{i z} n_{g}\right)}=\left(1+\frac{v_{i}}{\gamma D_{A 2}} \operatorname{coth} \frac{\gamma h}{2}\right) \frac{n_{i 2}}{n_{c 2}}
$$

since $K_{i z c}$ and $n_{i 2} / n_{c 2}$ depend on the DL potential drop, $V_{s}$, solving Eqn. (5.26) numerically determines the DL strength needed for the particle balance upstream. The numerical solutions of variation of the DL strength with pressure were compared to results from two different experiments, as shown in Fig. (5.6). It is seen that the theoretical model successfully accounts for both the upper and the lower pressure thresholds for DL formation in both experimental devices. 


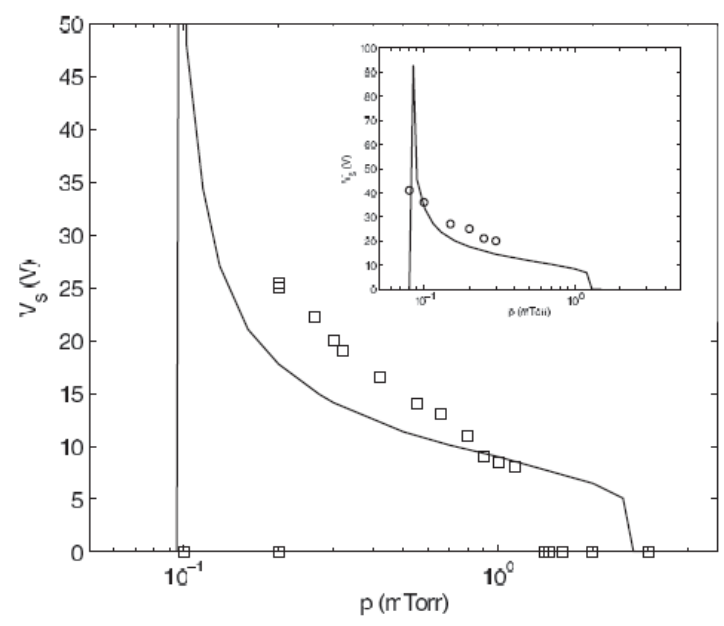

Figure 5.6. Pressure variation of the DL strength, $V_{s}$ : Theoretical (solid line) and experimental (open squares) results for Chi-Kung. ${ }^{39}$ The inset shows similar results for a larger helicon device: WOMBAT. ${ }^{57}$ Figure from Ref. [52].

The accelerated ions enter the expansion chamber downstream, where they undergo charge transfer collisions with the neutral gas, thereby producing thermal ions. The radius of the ion beam inside the expansion chamber is typically the same as the source radius. The neutralizing electrons associated with the downstream ion beam also lead to additional ionization. To describe this process, a simple diffusion model is used, where the ionization and the volume rate of thermal ion production due to the ion beam are assumed to be uniform inside the expansion chamber. The downstream ion beam density in the model is

$$
n_{b}=n_{b 1} \mathrm{e}^{-(z-h) / \lambda_{b}} \quad \text { for } \quad h<z<h+w \text { and } r<R_{2}
$$

where $\lambda_{b} \approx 0.7 \lambda_{i}$ is the mean free path of the ion-neutral charge transfer in the ion beam. The net number of ions in the beam converted to thermal ions per second in the expansion chamber is given by $n_{b 1} v_{b}\left(1-e^{-w / \lambda_{b}}\right)$, where $v_{b}=\left(v_{i}^{2}+2 e V_{s} / M\right)^{1 / 2}$ is the ion beam velocity in the expansion chamber. Dividing by the volume of the expansion 
chamber, we get the volume rate of production of the thermal ions $\left(v_{b} n_{b_{\text {eff }}}\right)$, where $v_{b}=v_{b} / \lambda_{b}$ is the charge transfer frequency and

$$
n_{b_{\text {eff }}}=n_{b 1} \frac{\lambda_{b}}{w} \frac{R_{2}^{2}}{R_{1}^{2}}\left(1-e^{-w / \lambda_{b}}\right)
$$

The diffusion equation for the thermal ions downstream is

$$
-D_{A 1} \frac{d^{2} n_{i}}{d z^{2}}+v_{R_{1}} n_{i}=n_{g} K_{i z}\left(n_{i}+n_{b_{\text {eff }}}\right)+v_{b} n_{b_{\text {eff }}}
$$

where $D_{A 1}=h_{R_{1}} u_{B} R_{1}$ and $v_{R_{1}}=2 h_{R_{1}} u_{B} / R_{1}$ is the downstream radial loss frequency. The solution to the diffusion equation is:

$$
n_{i}=n_{a 1} \cos k_{1}(z-h)+\alpha n_{b_{\text {eff }}}\left[\cos k_{1}(z-h)-1\right]
$$

where $k_{1}^{2}=\left(n_{g} K_{i z}-v_{R_{1}}\right) / D_{A 1}$ is the square of the axial wave-number, and

$$
\alpha=\frac{k_{1}^{2} D_{A 1}+v_{R_{1}}+v_{b}}{k_{1}^{2} D_{A 1}}
$$

Setting the thermal ion flux $\Gamma_{i}=n_{i} u_{B}$ at $z=h+w$,

$$
k_{1} D_{A 1}\left(n_{a 1}+\alpha n_{b_{\text {eff }}}\right) \sin k_{1} w=u B\left(n_{a 1}+\alpha n_{b_{\text {eff }}}\right) \cos k_{1} w-u_{B} \alpha n_{b_{\text {eff }}}
$$

Solving Eqn. (5.32) for $k_{1}$, the density profile can be obtained from Eqn. (5.30). $k_{1}$ is found to be real for low pressures and imaginary for higher pressures. The potential distribution within the DL can be obtained from the density distribution. Fixing the zero of the potential at the downstream wall and a floating potential of 
$V_{f 1}=\frac{1}{2} T_{e} \ln (2 M / \pi m)=5.4 T_{e}$ across the sheath there, ${ }^{58}$ the downstream and the upstream potentials, $V_{1}(z)$ and $V_{2}(z)$ become

$$
\begin{gathered}
V_{1}(z)=5.4 T_{e}+T_{e} \ln \left(\frac{n_{e}(z)}{n_{e}(h+w)}\right) \\
V_{2}(z)=V_{1}(h)+V_{s}+T_{e} \ln \left(\frac{n_{d 2}(z)}{n_{d 2}(h)}\right)
\end{gathered}
$$

The potential drop $V_{f 2}$, across the sheath at the upstream wall is obtained by equating the ion and electron fluxes at the wall,

$$
n_{i 2} V_{i}=\frac{1}{4}\left(\frac{8 e T_{e}}{\pi m}\right)^{1 / 2}\left(n_{d 2}+n_{c 1} e^{V_{s} / T_{e}}\right) e^{-V_{f 2} / T_{e}}
$$

Fig. (5.7) show the plasma potential and the thermal ion densities from the theoretical model on the left and published experimental results on the right ${ }^{39}$ for operating pressure of 0.2 mTorr. The general shape of the potential and the thermal ion density is quite similar to what is observed experimentally. One of the requirements of this model is the presence of accelerated electrons in the helicon source of about $20 \%$ of the total upstream density. Even though some studies with Langmuir probe data give a vague indication of an electron beam, ${ }^{41}$ other experiments on different devices indicate a very weak electron beam ${ }^{59}$ or no electron beam at all. ${ }^{60}$ A key prediction of this model is that the formation of a DL should be accompanied with a distinct increase in the upstream density, due to enhanced ionization by the electron beam being accelerated towards the helicon source. 

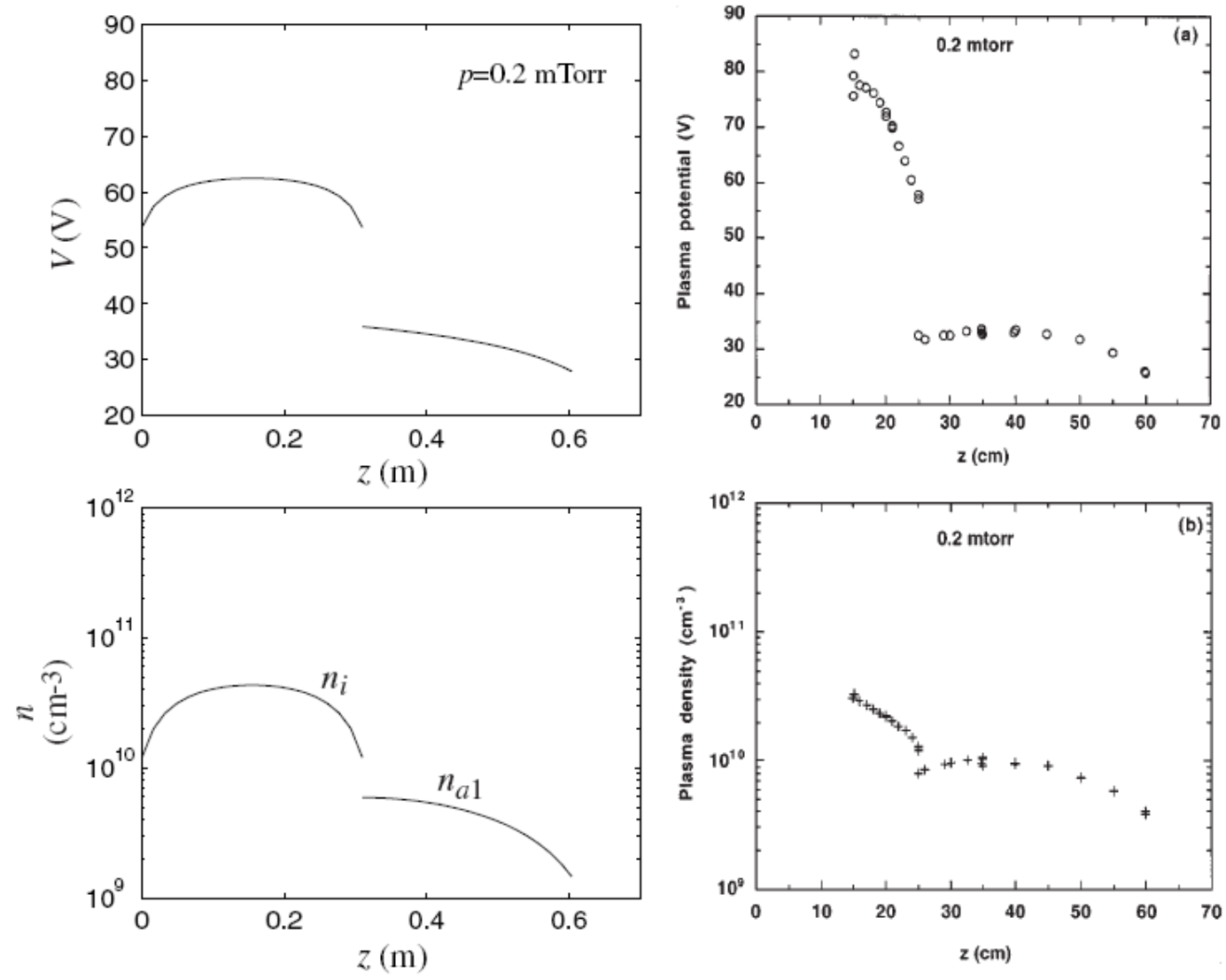

Figure 5.7. Potential (top panes) and thermal ion density (bottom panes) with position at 0.2 mTorr of pressure. The left hand panes are the results from the theoretical model by Lieberman while the right hand panes are observed experimentally. Figure from Ref. [51]

In the context of plasma propulsion, an alternate theory of current-free DL was proposed in Ref. [53], where the thrust is imparted to the plasma at the DL itself by the magnetic field pressure. In the model of Ref. [52], the thrust is not imparted to the plasma at the DL but only at the back wall, where the electrons accelerated towards the source by the DL are reflected back.

\subsection{Experimental Results}

Experimental studies in which the neutral pressure was varied $^{39,43,44,57}$ demonstrated a clear pressure threshold for ion beam formation that is consistent with the predictions of the diffusion-controlled model. However, because the overall neutral pressure was also changing in those studies, it was difficult to demonstrate that changes 
in the ratio of the upstream to downstream density $\left(D_{R}=n_{2} / n_{1}\right)$ were solely a result of the formation of the current-free DL - a key prediction of the model. The substantially different magnetic field profiles in another recent study ${ }^{61}$ that demonstrated a threshold in magnetic field strength for DL formation also made clear identification of the mechanism responsible for the observed change in $\mathrm{D}_{\mathrm{R}}$ problematic.

Here we describe our observations of the effect of changing only the antenna frequency on the formation of the ion beams downstream of a low pressure expanding helicon argon plasma. ${ }^{62,63,64}$ All other source parameters: the magnetic field in the source and expansion chambers, the power that the antenna feeds into the plasma, and the neutral gas pressure (the gas flow rate and the rate of pumping at both the HELIX and the LEIA ends), were held fixed. The plasma density, electron temperature, and parallel ion velocity distribution function IVDFs were measured both upstream and downstream of the acceleration region. The IVDFs were obtained by both retarding Field Energy Analyzer ${ }^{40,65}$ and laser induced fluorescence. ${ }^{66}$ Analysis of the RFEA measurements yields ion beam energies and relative ion beam to background ion densities that are consistent with the LIF measurements. The electron temperature and plasma densities upstream and downstream of the DL were measured with rf compensated Langmuir probes. ${ }^{67}$ An electrostatic double probe was used to measure the frequency spectrum of the electric field fluctuations for plasmas with and without a stable double layer. It is found that the ion beam, and hence the DL, is not observed for certain frequencies of the source operation. The electrostatic fluctuation measurements point to a beam-driven, ion acoustic instability as the mechanism responsible for suppression of the DL at low antenna frequencies. Fig. 5.8 shows the positions of the different diagnostics used in this study, with respect to the HELIX-LEIA chamber, the helicon source antenna and the magnetic field gradient. 


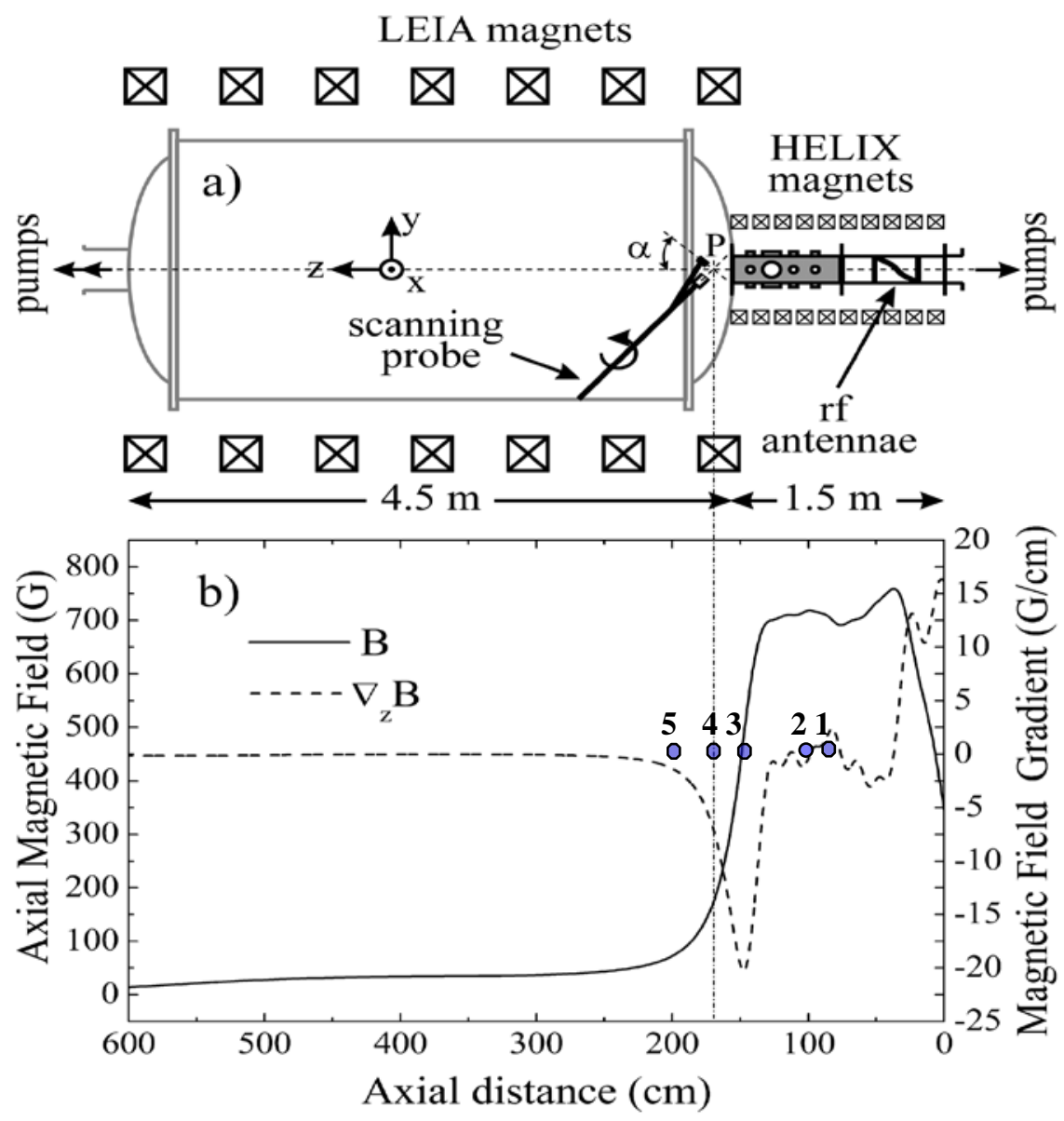

Figure 5.8. The HELIX-LEIA experimental device and the magnetic field profile (continuous line) and the profile of the magnetic field gradient (dashed line), which is a maximum near the junction of HELIX and LEIA. The upstream Langmuir probe is at position 1, the double probe is placed at position 2, the upstream LIF signal is collected at position 3, the downstream Langmuir probe and the downstream LIF measurements are taken at position 4, and the RFEA is located at position 5.

\subsubsection{Threshold for Ion Beam Formation}

Both the downstream IVDF measurements and the upstream and downstream plasma densities and electron temperatures were obtained for argon plasma at an rf power of $750 \mathrm{~W}$, a magnetic field of $700 \mathrm{G}$ in the source, and a magnetic field of $50 \mathrm{G}$ in the expansion region. With a constant flow rate of $3.6 \mathrm{sccm}$ into the system, the neutral pressures in the source and expansion regions were 0.2 mTorr and 0.05 mTorr, respectively. To obtain sufficient signal-to-noise for the upstream LIF-based IVDF 
measurements (using collection optics external to the vacuum chamber), the magnetic field in the expansion region was decreased to $14 \mathrm{G}$, the rf power lowered to $700 \mathrm{~W}$, and the flow rate increased to $8.6 \mathrm{sccm}$; yielding neutral pressures in the source and expansion regions of 1.0 mTorr and 0.09 mTorr respectively. To optimize the RFEA measurements (which was placed further away from the HELIX-LEIA junction), the flow rate was reduced to $5 \mathrm{sccm}$; yielding neutral pressures in the source and expansion regions of 0.7 mTorr and 0.07 mTorr respectively. The double probe measurements were obtained at the same source parameters as those used for the RFEA measurements. Note that during the scans of the antenna frequency, all other source parameters were held constant.

LIF measurements of the Doppler shifted downstream IVDF (relative to the rest frame absorption line frequency) are shown in Fig. 5.9. Because the laser injection direction is opposite to the flow of the plasma, a negative frequency shift corresponds to ion flow towards the expansion chamber. The frequency shift is proportional to the velocity of the ions, where the wavelength of the injected laser $(\lambda=661.6616 \mathrm{~nm}$ in these experiments) is the constant of proportionality. Hence the velocity (in $\mathrm{m} / \mathrm{s}$ ) is given by the product of the frequency shift (in GHz) and the laser wavelength (in $\mathrm{nm}$ ). The large amplitude peaks at low velocities (small laser frequency shift) are the background ion population. For antenna frequencies below $11.5 \mathrm{MHz}$, there is a strong background population and a long tail of energetic ions. For antenna frequencies of $12 \mathrm{MHz}$ and higher, a distinct ion beam appears at large, downstream directed (negative frequency shift), velocities. Based on fits to a pair of drifting Maxwellian distributions and correcting the measured bulk flow speeds for the angle $\alpha$ of the probe $\left(\mathrm{V}_{\text {actual }}=\right.$ $\mathrm{V}_{\text {obs. }} / \cos (\alpha) ; \alpha=47^{\circ}$ ) at location 4 in Fig. 5.8, the ion beam velocity increases from approximately $7.7 \mathrm{~km} / \mathrm{s}$ at $13.56 \mathrm{MHz}$ to $9.0 \mathrm{~km} / \mathrm{s}$ at $12 \mathrm{MHz}$. Since previous measurements demonstrated that the beam velocity increases for weaker expansion chamber magnetic fields ${ }^{68}$, we expect that the downstream beam velocities would have been even larger had the expansion chamber magnetic field been reduced to the $14 \mathrm{G}$ similar to what was used for the upstream IVDF measurements described later. 


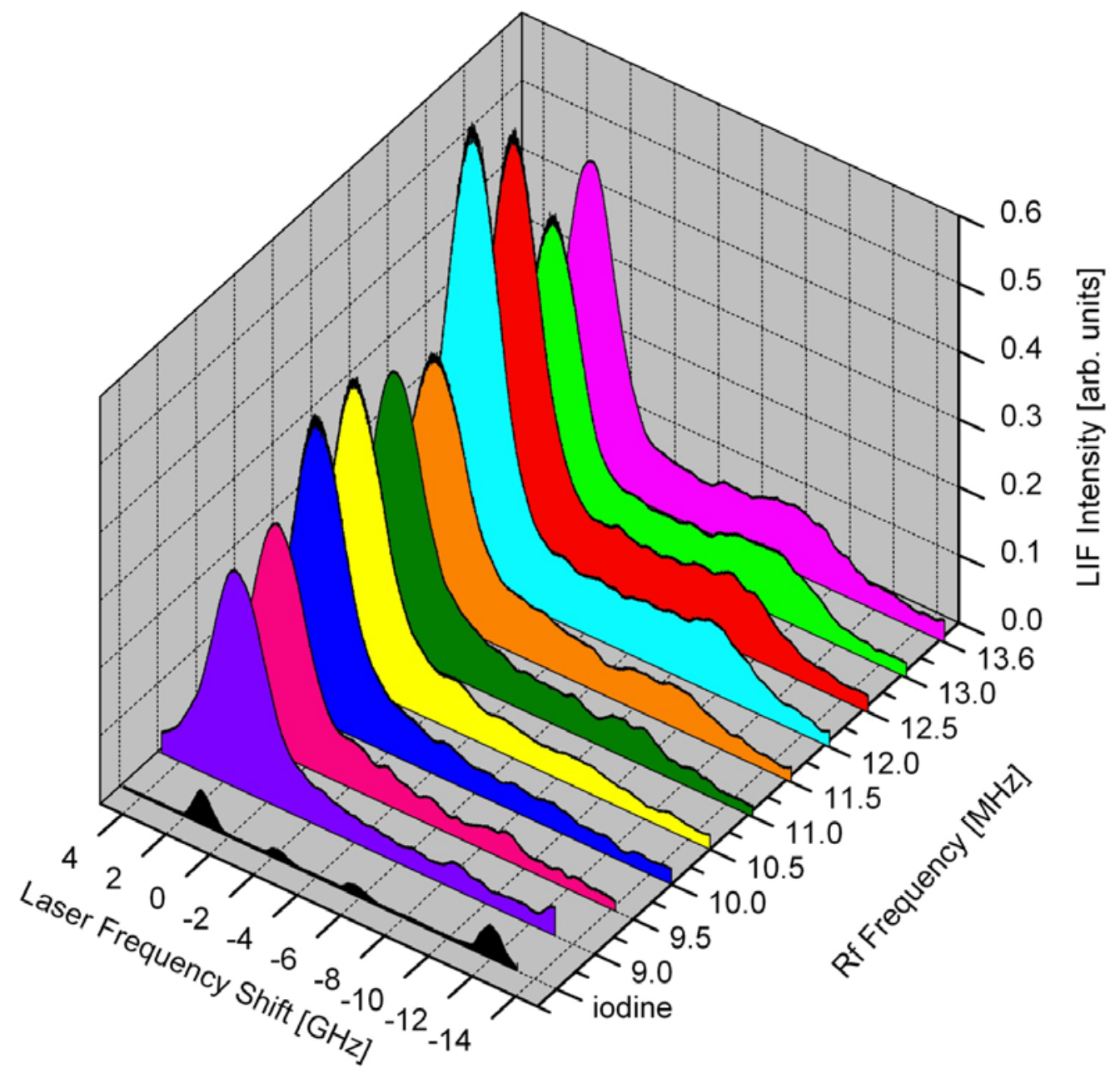

Figure 5.9. LIF measurements of the downstream IVDF versus antenna frequency obtained 124 $\mathrm{cm}$ downstream of the antenna. The laser frequency shift in $\mathrm{GHz}$ is the Doppler shift due to the velocity of the ions. The reference iodine line is also shown. The distinct fast ion population, the ion beam, is found only for the higher antenna frequencies. 


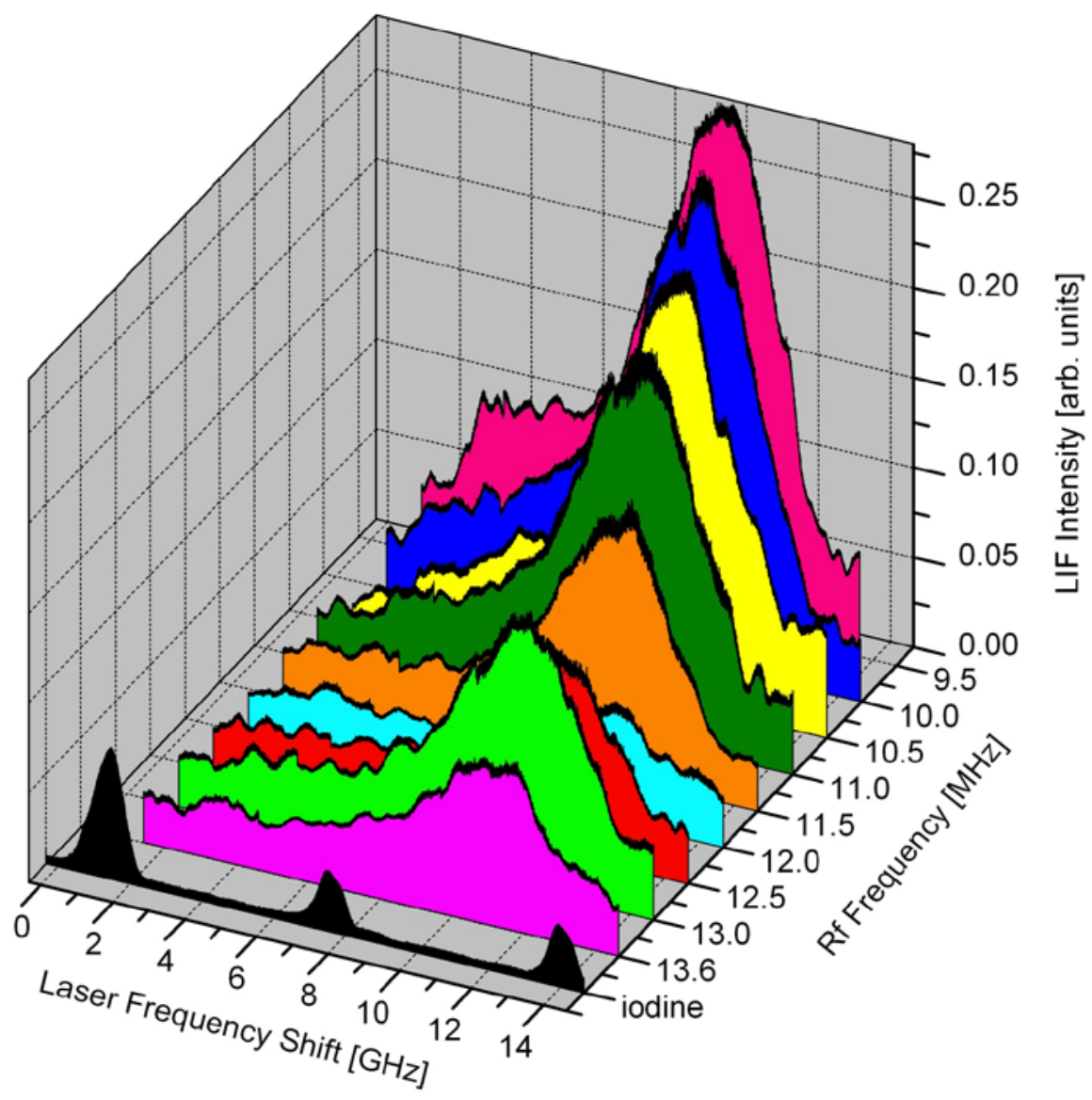

Figure 5.10. LIF measurements of the upstream IVDF versus antenna obtained $95 \mathrm{~cm}$ downstream of the antenna. The laser frequency shift in $\mathrm{GHz}$ is the Doppler shift due to the velocity of the ions. The reference iodine line is also shown. A broad tail due to slower ion population is present only for the lower antenna frequencies.

Shown in Fig. 5.10 are the upstream LIF measurements of the IVDF. A positive shift in the frequency of the peak LIF emission corresponds to ion flow towards the expansion chamber. Note that the antenna frequency axis has been reversed compared to Fig. 3 so that the entire IVDF can be seen for each measurement. After correcting for the Zeeman shift of the absorption line for a magnetic field of $700 \mathrm{G}$, there is a slight increase in the downstream-directed drift velocity of the bulk ion population with increasing antenna frequency, from $6.7 \mathrm{~km} / \mathrm{s}$ at $9.5 \mathrm{MHz}$ to $7.5 \mathrm{~km} / \mathrm{s}$ at $12 \mathrm{MHz}$. The bulk drift velocity remains nearly constant for further increases in the antenna frequency. The 
increase in LIF signal at lower antenna frequencies likely results from an increase in the population of the initial metastable argon ion state of the LIF sequence (since lower antenna frequencies typically result in improved coupling of the rf power into the plasma) or the slightly longer time spent in front of the collection optics by slower moving excited state ions. Note that for antenna frequencies below $11.5 \mathrm{MHz}$, a broad tail of slower ions appears. By 9.5 MHz, the tail of slow ions expands to include ions moving in the upstream direction as well. The increase in the drift velocity with increasing antenna frequency is opposite to what was observed in case of the ion beam from the downstream LIF measurements.

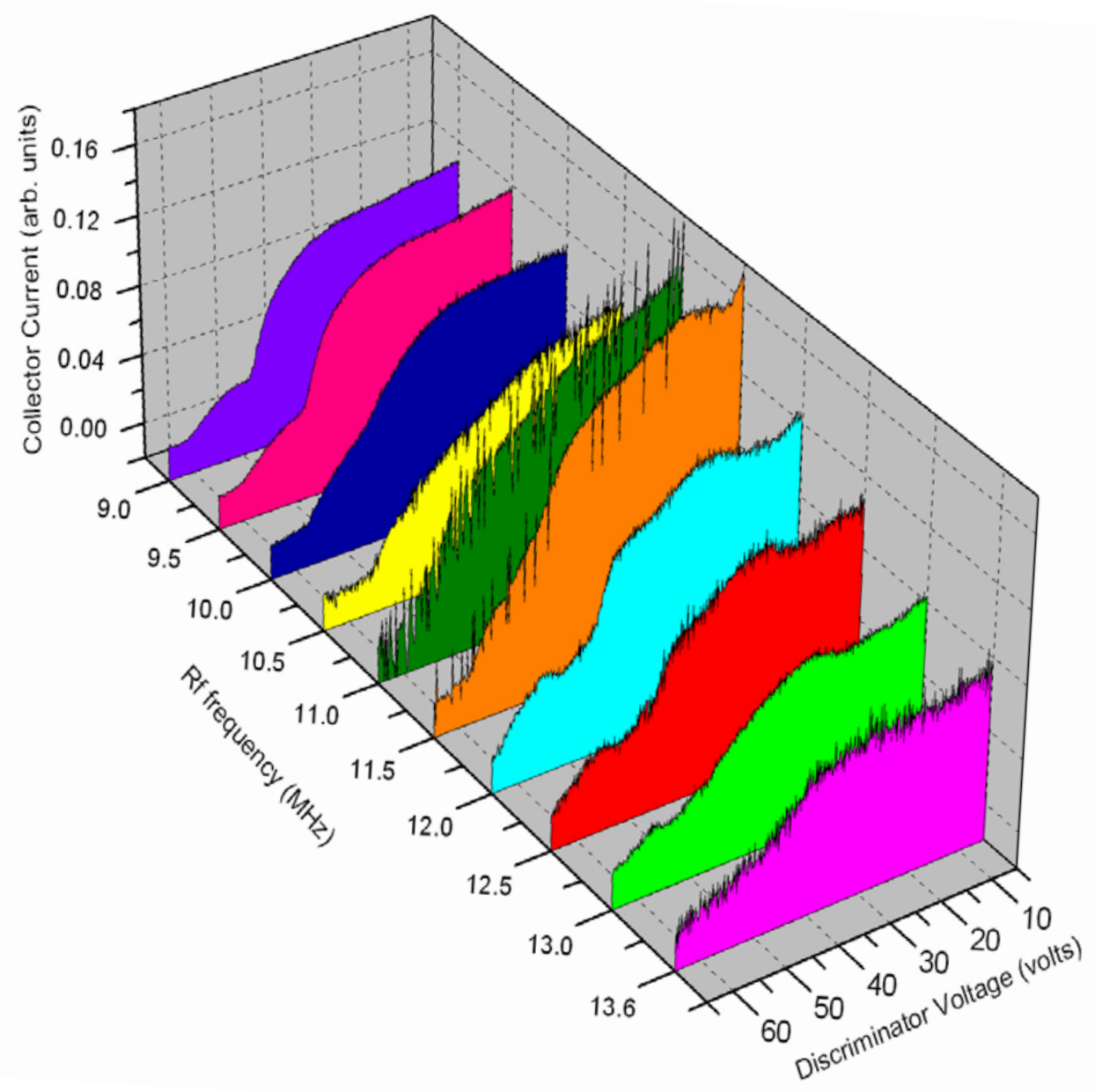

Figure 5.11. RFEA collector current versus discriminator voltage as a function of the antenna frequency obtained $184 \mathrm{~cm}$ downstream of the antenna. 


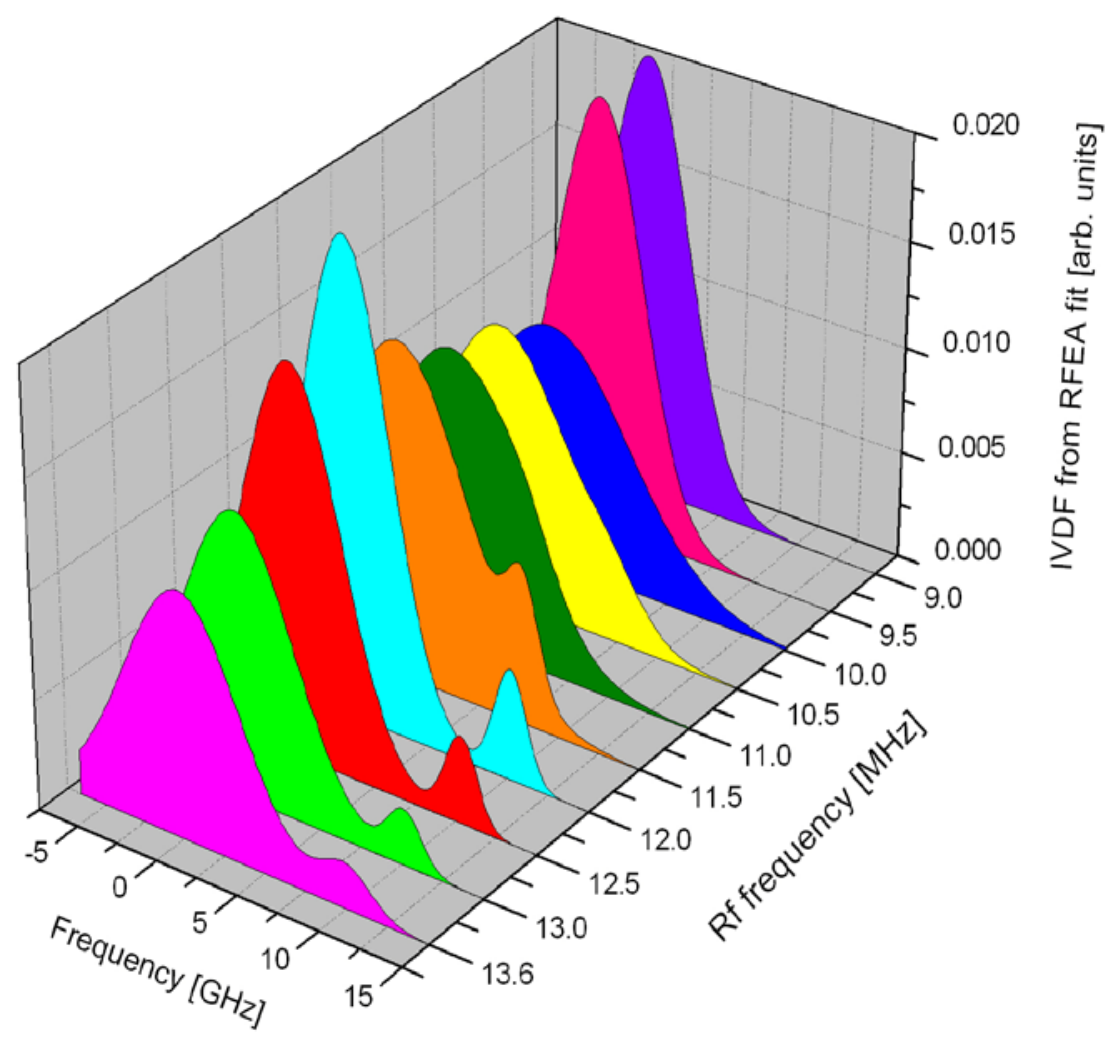

Figure 5.12. RFEA measurements (the bi-Maxwellian which gives the best fit of Eq. 4.1 to the raw data in Fig. 5.11) of the downstream IVDF versus antenna frequency obtained $184 \mathrm{~cm}$ downstream of the antenna. The ion beam appears only for the higher antenna frequencies.

Shown in Fig. 5.11 are RFEA collector current measurements versus discriminator voltage for the same range of antenna frequencies. The data shown were averaged over 100 measurements at each antenna frequency. There is a clear onset of large amplitude fluctuations in the RFEA collector current at driving frequencies of 11.0 $\mathrm{MHz}$ to $11.5 \mathrm{MHz}$. This 11.5 MHz threshold corresponds to the same threshold frequency above which two distinct ion populations are evident in the RFEA data: one is the background ion population that appears in the RFEA data at the plasma potential of approximately $25 \mathrm{~V}$ and the other is an ion beam population that appears at high discriminator voltages. At lower antenna frequencies, only the background ion population appears in the RFEA data. The parent ion velocity distribution functions obtained from theoretical fits Eq. 4.1 to the data of Fig. 5.11 are shown in Fig. 5.12. The RFEA-derived 
IVDFs are completely consistent with the downstream LIF IVDF measurements. For antenna frequencies below $11.5 \mathrm{MHz}$, there is a single, low velocity, background ion population. For antenna frequencies above $12 \mathrm{MHz}$, a distinct ion beam appears at large, downstream-directed velocities. From the fits to the RFEA measurements, the ion beam velocity increases from approximately $5.5 \mathrm{~km} / \mathrm{s}$ at $13.56 \mathrm{MHz}$ to $6.4 \mathrm{~km} / \mathrm{s}$ at $12 \mathrm{MHz}$. The roughly $1 \mathrm{~km} / \mathrm{s}$ increase in the ion beam velocity matches the trend seen in the downstream LIF measurements.

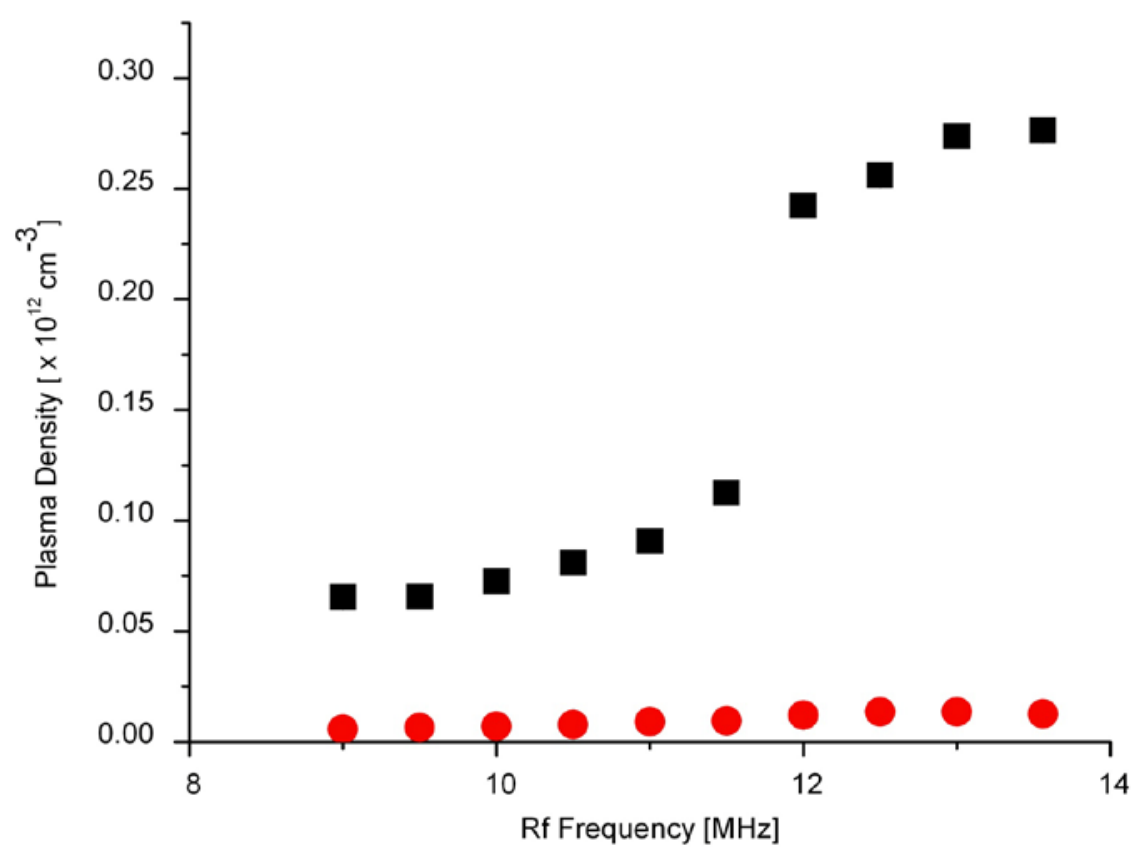

Figure 5.13. Upstream (black squares) and downstream (red circles) plasma density versus antenna frequency for rf power of $750 \mathrm{~W}, \mathrm{~B}_{\mathrm{HELX}}=700 \mathrm{G}, \mathrm{B}_{\mathrm{LEIA}}=50 \mathrm{G}$ and a pressure of 0.2 mTorr. The error bars are smaller than the size of the data points shown.

The upstream and downstream plasma densities are shown in Fig. 5.13. There is a gradual increase in the downstream plasma density with increasing antenna frequency, from $6 \times 10^{9} \mathrm{~cm}^{-3}$ at $9 \mathrm{MHz}$ to $1 \times 10^{10} \mathrm{~cm}^{-3}$ at $13.56 \mathrm{MHz}$. Upstream of the expansion region; there is a large and discontinuous increase in the measured density as the antenna frequency changes from $11.5 \mathrm{MHz}$ to $12 \mathrm{MHz}$. This is the same driving frequency at which the downstream ion beam, and by implication the DL, appears. With the DL present (at the higher antenna frequencies), the density increased to $2.6 \times 10^{11} \mathrm{~cm}^{-3}$ from 
an average density of $0.85 \times 10^{11} \mathrm{~cm}^{-3}$ without the DL; an increase of nearly a factor of three. It is important to remember that this increase in plasma density occurs without any change in the magnetic field geometry, neutral pressure, or forward rf power.

The upstream and downstream electron temperatures as measured with the same Langmuir probes also show a distinct change in the upstream electron temperature at driving frequencies above $12 \mathrm{MHz}$. The electron temperature drops from approximately $10.5 \mathrm{eV}$ when the $\mathrm{DL}$ is not present to an average of $8.7 \mathrm{eV}$ for driving frequencies at which the ion beam is observed downstream. The downstream electron temperature is relatively constant at an average value of $7.9 \mathrm{eV}$ for all driving frequencies.

The IVDF and density measurements are consistent with the diffusion-controlled model prediction of enhanced upstream plasma density arising from the formation of a current-free DL in expanding plasma. ${ }^{51,52,53}$ For identical plasma source parameters, apart from the driving frequency, the upstream density clearly increases when the downstream LIF and RFEA measurements indicate the presence of a current-free DL.

During these experiments, it was noticed that the upstream and downstream Langmuir probe measurements were extraordinarily noisy for source frequencies up to 11.5 MHz. Each measurement had to be repeated many times to obtain reliable density and electron temperature measurements. Above source frequencies of $12 \mathrm{MHz}$, a single measurement was sufficient. Shown in Fig. 5.14 are two typical rf compensated Langmuir probe I-V curves that were obtained for two different driving frequencies.

To quantify this phenomenon, the average root-mean-squared deviation from a linear fit to a $6.0 \mathrm{~V}$ wide region of the Langmuir probe $\mathrm{I}-\mathrm{V}$ curve in the electron retardation region ${ }^{69}$ was calculated as a function of rf frequency and the results normalized to the average value of the reference I-V curve in that same region, are shown in Fig. 5.15. Essentially, Fig. 5.15 is a measure of the noise-to-signal versus driving frequency. Note that these measurements were obtained after four Langmuir probe measurements were averaged together, i.e., the noise-to-signal is substantial, even after multiple measurements are averaged together. These data indicate that coincident with the appearance of the current-free DL, there is a dramatic reduction in the electrostatic noise both upstream and downstream of the expansion region. The presence of these large electrostatic fluctuations in the Langmuir probe traces, at lower antenna frequencies, 
motivated us to install a double probe to measure the frequency spectrum of the electric field fluctuations in the plasma.
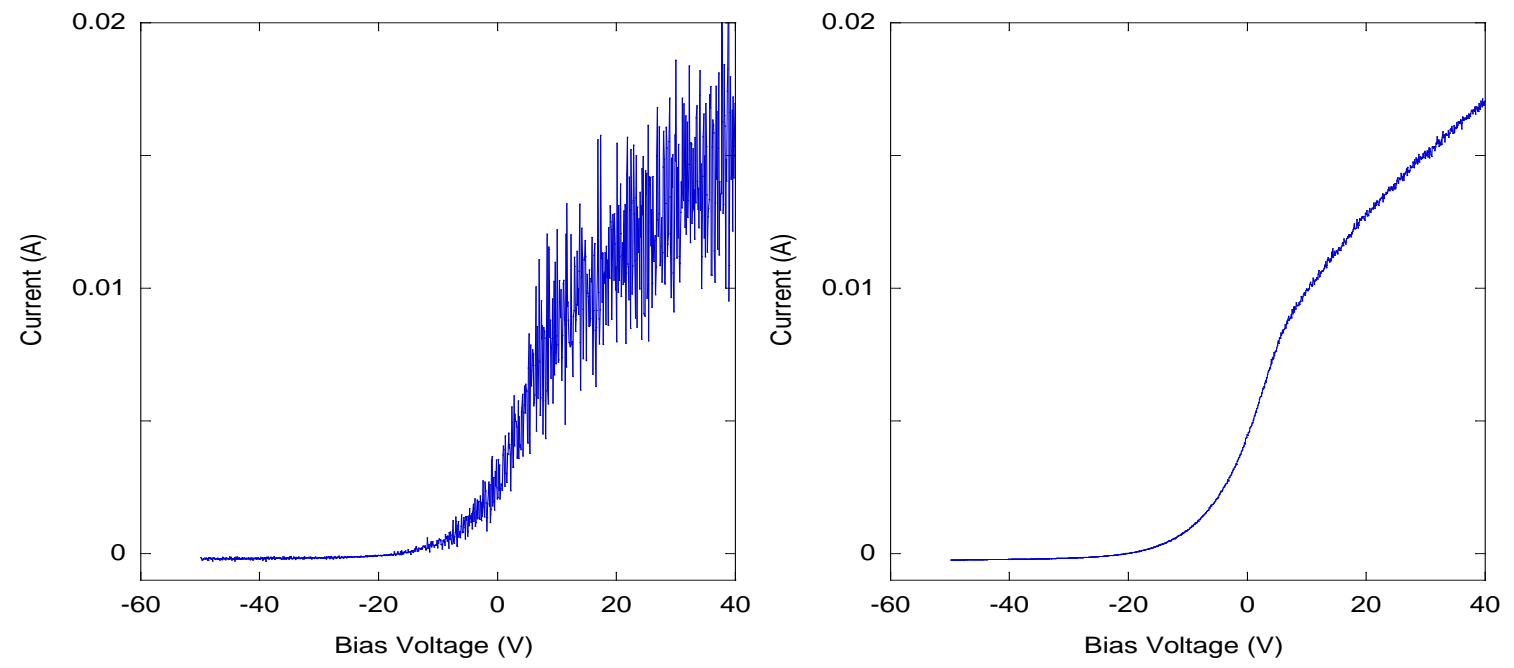

Figure 5.14. Typical rf compensated Langmuir probe traces for two representative antenna frequencies. (a) The $9.5 \mathrm{MHz}$ antenna frequency trace shows large scale fluctuations even after many averages. (b) The single $13.56 \mathrm{MHz}$ antenna frequency Langmuir probe trace is devoid of any electrostatic fluctuations.

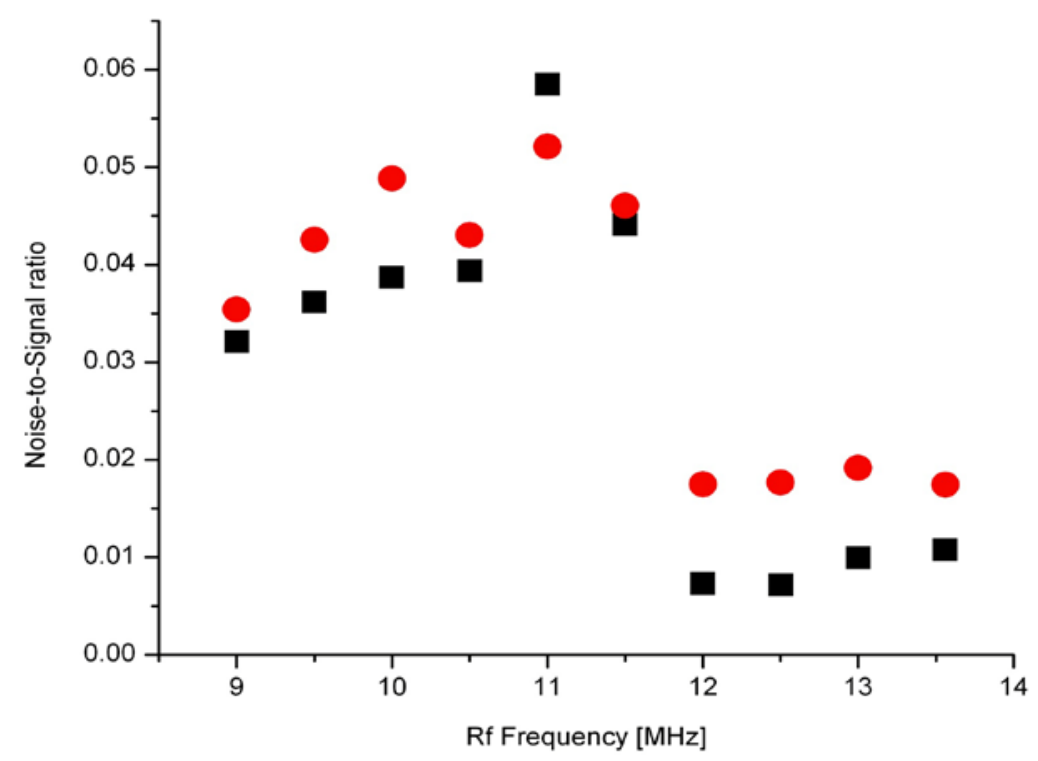

Figure 5.15. Upstream (black squares) and downstream (red circles) noise-to-signal ratio versus antenna frequency. The decrease in the noise for higher antenna frequencies corresponds to the appearance of the ion beam. 
Shown in Fig. 5.16 are the double probe measurements of the power spectra of the upstream electric field fluctuations versus driving frequency. For driving frequencies below $11.5 \mathrm{MHz}$, the spectra are dominated by a wave at a fundamental frequency of $17.5 \mathrm{kHz}$ and its harmonics. Fig. 5.17 shows the power spectra of the same electrostatic fluctuations near the primary antenna frequency for different antenna frequencies. The driving antenna frequency beats with the wave at $17.5 \mathrm{KHz}$ and produces sidebands only when the antenna frequency is less than $11.5 \mathrm{MHz}$. The wave activity decreases in intensity as the rf antenna frequency is reduced from $13.56 \mathrm{MHz}$ to $9 \mathrm{MHz}$. Expanded views of the power spectra for antenna frequencies of $9 \mathrm{MHz}$ and $12 \mathrm{MHz}$ are shown in Fig. 5.18 a and 5.18b, respectively.

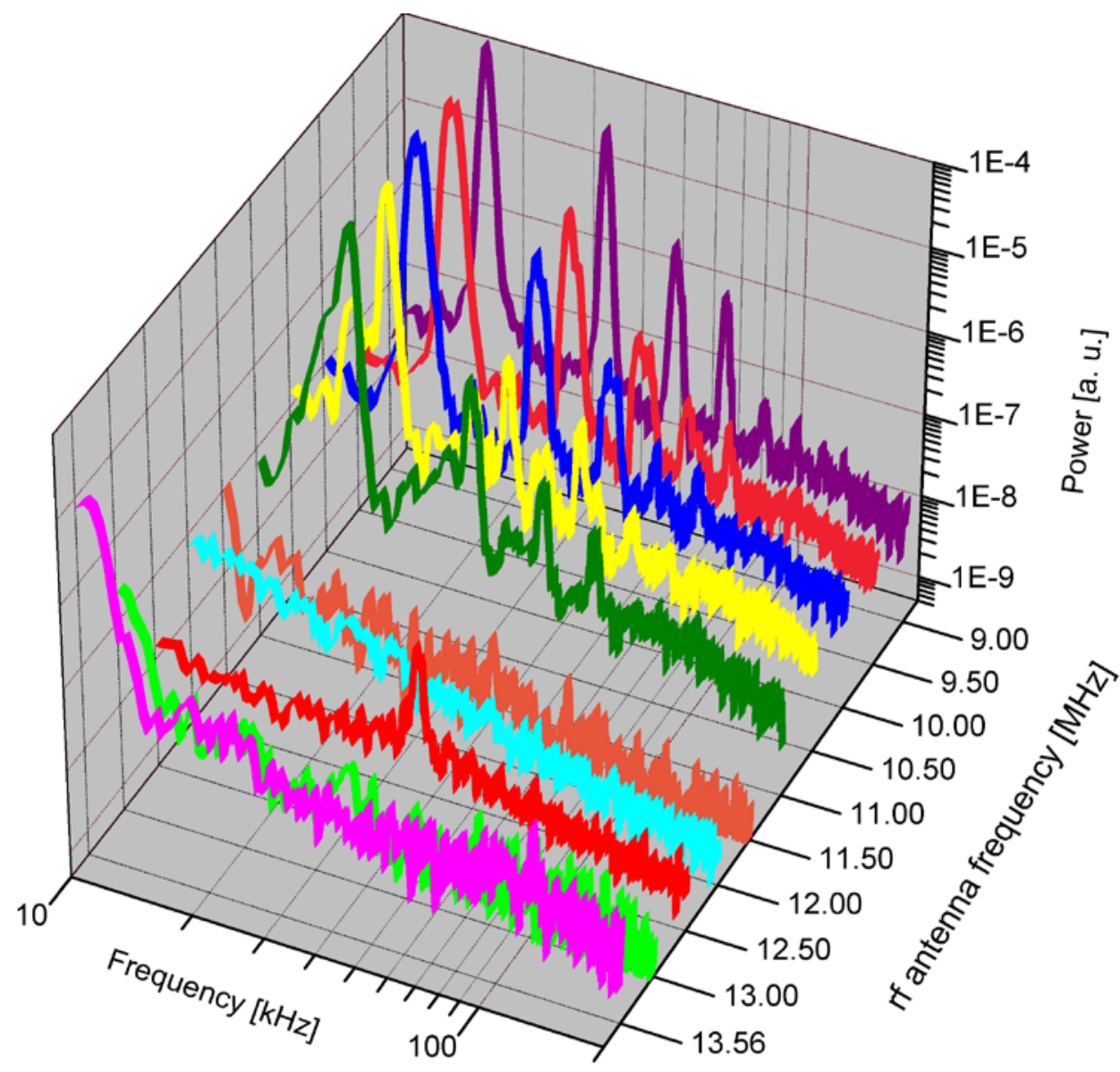

Figure 5.16. Double probe measurements of the power spectrum of the electrostatic instabilities versus antenna frequency obtained $50 \mathrm{~cm}$ downstream of the antenna. The instability at $17.5 \mathrm{kHz}$ and its harmonics appears only for the lower antenna frequencies. 


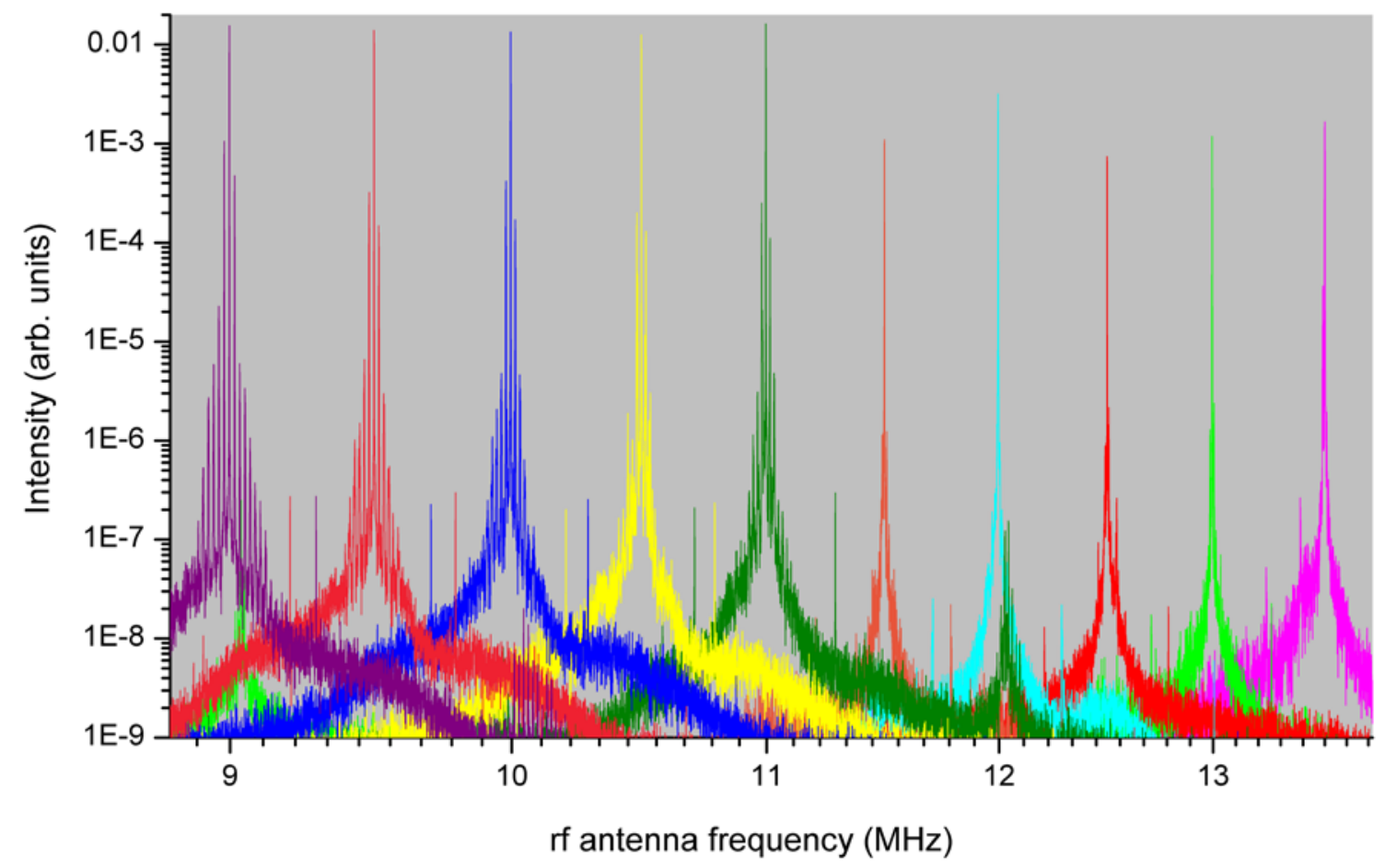

Figure 5.17. Power spectra of the electrostatic fluctuations near the primary antenna frequencies.
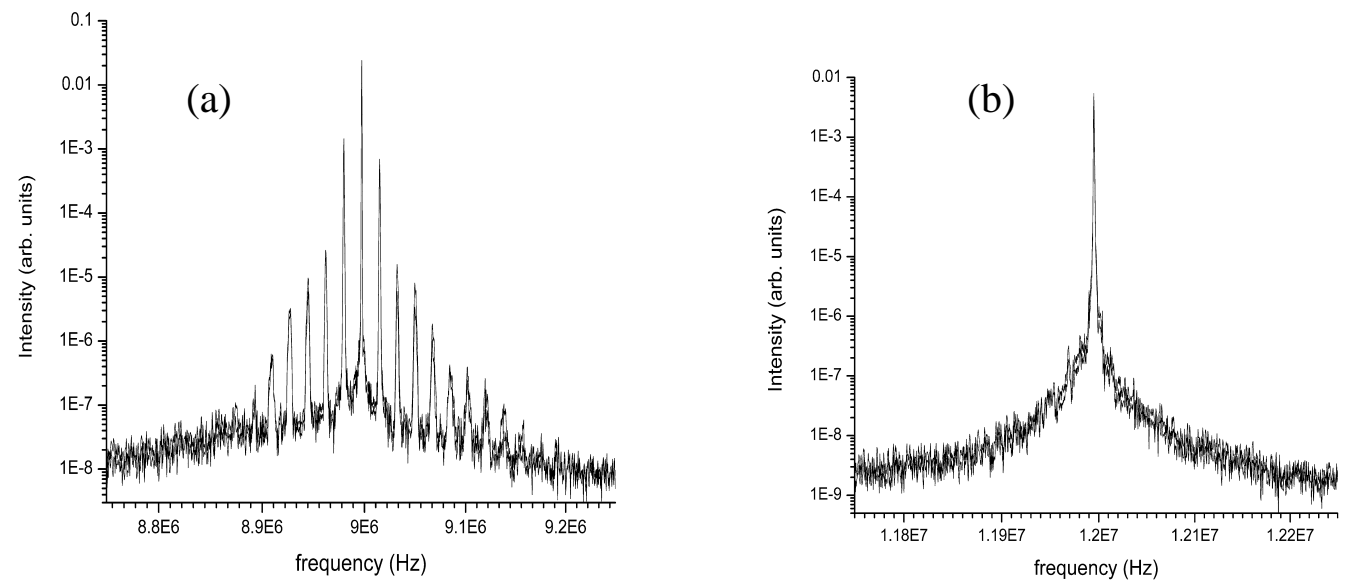

Figure 5.18. Expanded plots of the power spectra of the electrostatic fluctuations near the primary antenna frequencies for two runs: (a) $9 \mathrm{MHz}$ and (b) $12 \mathrm{MHz}$. 
These experimental results suggest that there is a threshold for the formation of a supersonic ion beam due to a DL in expanding magnetic field geometry. To summarize, a stable ion beam in continuous operating conditions is found for antenna frequencies of 12 $\mathrm{MHz}$ or more. Correspondingly, as per the predictions from the diffusion controlled theoretical model, there is a sharp increase in the upstream density for the same frequencies. Below the threshold of clear ion beam formation, the electrostatic probe signals are very noisy. The electrostatic noises arise from large amplitude waves at a frequency of $17.5 \mathrm{kHz}$. The threshold effect provide a unique means of turning the DL on or off without changing the source parameters; magnetic field geometry, neutral gas pressure or flow; and forward rf power to the antenna.

\subsubsection{Nature of the Instability}

The $17.5 \mathrm{kHz}$ wave propagates primarily in the axial direction (determined by the rotation of the double probe) with a wavelength of approximately $30 \mathrm{~cm}$. Axial propagation rules out drift waves as the source of the instabilities. The short parallel wavelength and multi-harmonic excitation are inconsistent with the characteristics of the ionization instability observed in another DL experiment. ${ }^{70}$ The multiple harmonics spanning frequencies below and above the ion cyclotron frequency rule out electrostatic ion cyclotron waves. Given that the wavelengths are much larger than the Debye length $\left(\lambda_{D}<1 \mathrm{~mm}\right)$ but much shorter than the system size $(\sim 6 \mathrm{~m})$, the fact that they propagate along the background magnetic field ( $k \square B$ ), and that excitation of the waves appears to be associated with a threshold particle drift velocity (ion beam speed) comparable to the ion sound speed ( $\sim 6 \mathrm{~km} / \mathrm{s}$ for these plasmas), the waves are most likely due to a beam driven ion acoustic instability. ${ }^{71}$ 


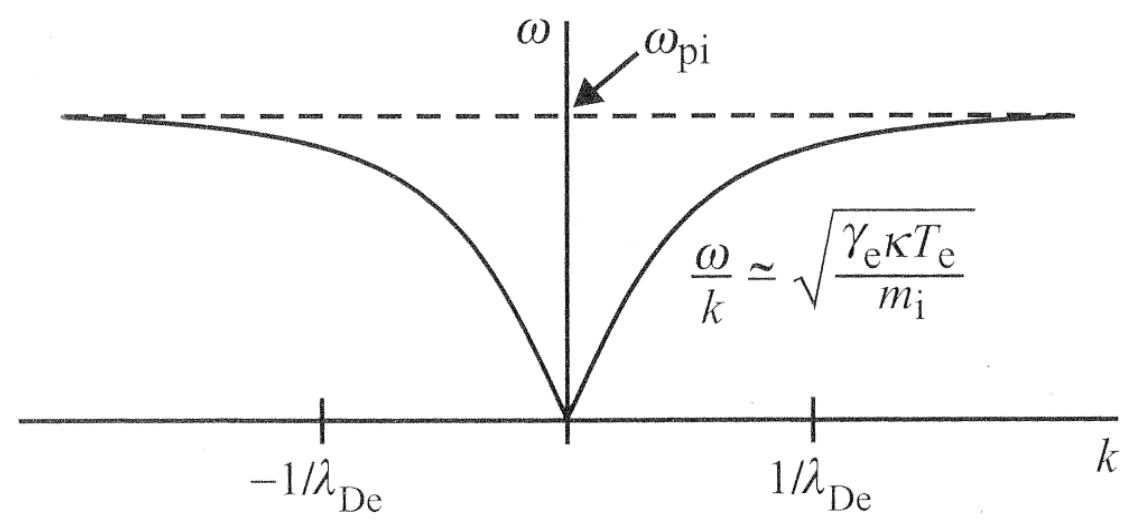

Figure 5.19. The full cold plasma dispersion relation for the ion acoustic mode, for $T_{e}<<T_{i}$. The figure is obtained from Ref. [72].

The dispersion relation for the ion acoustic mode, for ion temperatures much smaller than the electron temperature (in our experiment $T_{i} \sim 0.5 \mathrm{eV}$ and $T_{e} \sim 5 \mathrm{eV}$ ), is given by:

$$
\omega^{2}=\frac{1}{\left(1+\gamma \lambda^{2}{ }_{D e} k^{2}\right)}\left(\frac{\gamma K_{B} T_{e}}{m_{i}}\right) k^{2}
$$

where $\omega$ is the frequency of the wave, $k$ is the wavenumber, $\lambda_{D e}$ is the electron Debye length, $\gamma$ is the ratio of the specific heats, $K_{B}$ is the Boltzmann constant, $m_{i}$ is the mass of the ion and $T_{e}$ is the electron temperature. According to this dispersion relation for ion acoustic waves (as shown in Fig. 5.19) for large values of $k$, the frequency asymptotically approaches the ion plasma frequency. For frequencies much less than the ion plasma frequency ( $50 \mathrm{MHz}$, in this experiment) and for values of $k$ smaller than $1 / \lambda_{D e},(\sim 12$ $\mathrm{cm}^{-1}$ for our experimental conditions) the wave frequency is linear in $k$ and the slope of this linear portion (i.e. the phase velocity) is approximately given by the ion sound speed:

$$
\frac{\omega}{k}= \pm \sqrt{\frac{\gamma \kappa_{B} T_{e}}{m_{i}}}=V_{s_{\text {ion }}}
$$




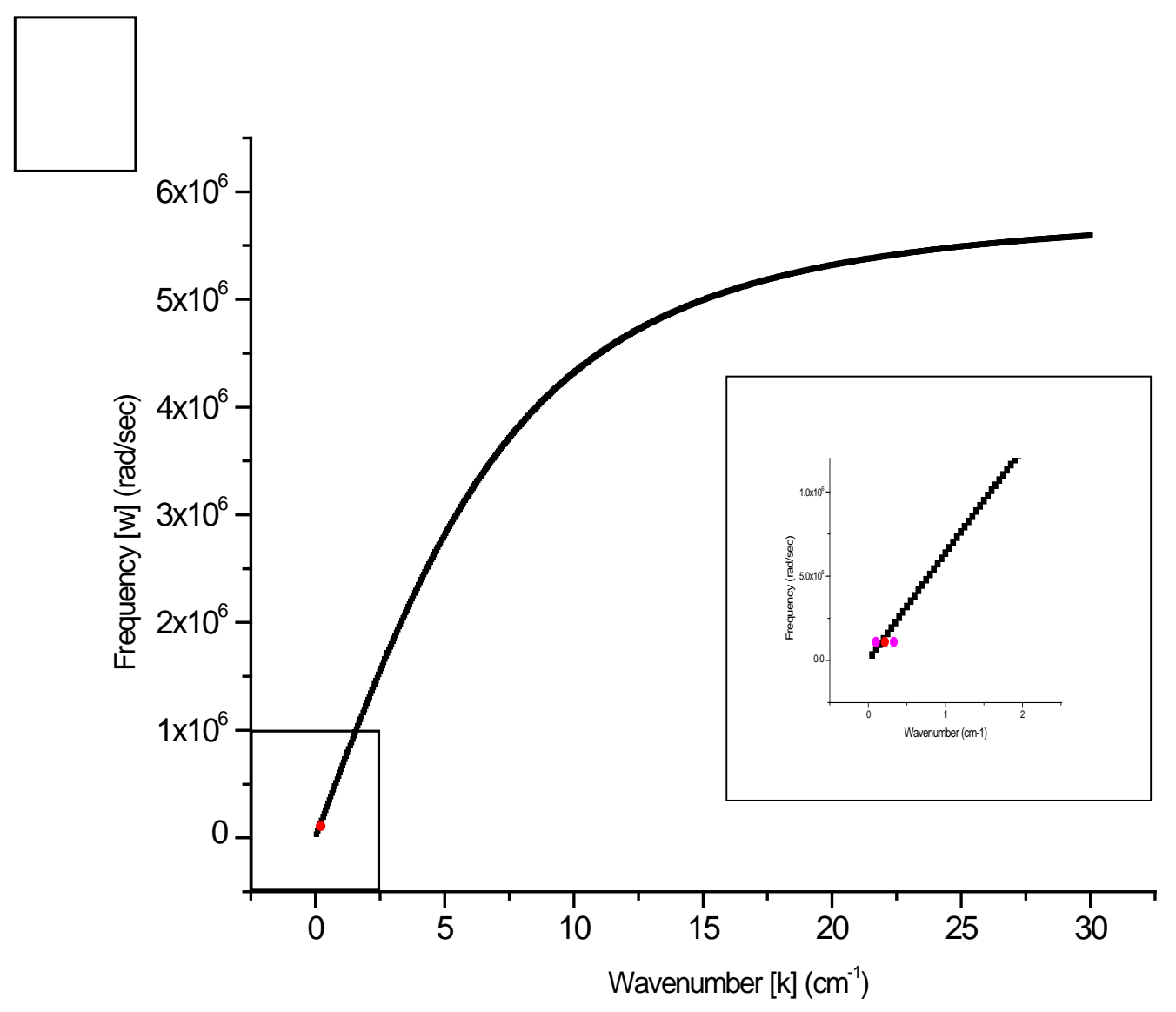

Figure 5.20. The positive root of the cold plasma dispersion relation (black squares) for the ion acoustic mode, calculated using the experimental parameters. The red circle is the measured value of $\omega$ and $k$ for the waves observed. The inset shows an enlarged portion of the plot, for relevant values of $k$, which also shows (in pink circles) the variance in the set of measurements.

For our experimental parameters, the calculated ion sound speed is $6.4 \mathrm{~km} / \mathrm{s}$. The measured average phase velocity of the $17.5 \mathrm{kHz}$ wave is $7 \pm 1 \mathrm{~km} / \mathrm{s}$. Thus, the observed waves are very likely ion acoustic waves. Since the waves naturally appear only at 17.5 $\mathrm{kHz}$, a full dispersion relation could not be obtained experimentally. When plotted along with the dispersion relation (Fig. 5.20), the wave frequency and wave number measurements fall on a single point that lies on the ion acoustic dispersion curve within experimental errors. 


\subsection{Discussion}

The instability at $17.5 \mathrm{kHz}$ and its harmonics appear only for driving frequencies below 11.5 MHz. These are the same driving frequencies for which large noise levels appear in the Langmuir probe traces and for which no ion beam is seen downstream in both the LIF and RFEA IVDF measurements. Moreover in the upstream LIF measurements, at the same antenna frequencies, there is a low density population of slow moving ions that extends from the drifting bulk population to zero velocity. Above the antenna frequency threshold of $11.5 \mathrm{MHz}$, the ion beam appears downstream of the plasma source and the beam velocity decreases with increasing driving frequency (see Fig. 5.21). The decrease in downstream ion beam velocity with increasing driving frequency suggests that the ion beam velocity would be even larger at lower driving frequencies if whatever mechanism that prevents ion beam formation did not arise at an antenna frequency of $11.5 \mathrm{MHz}$.

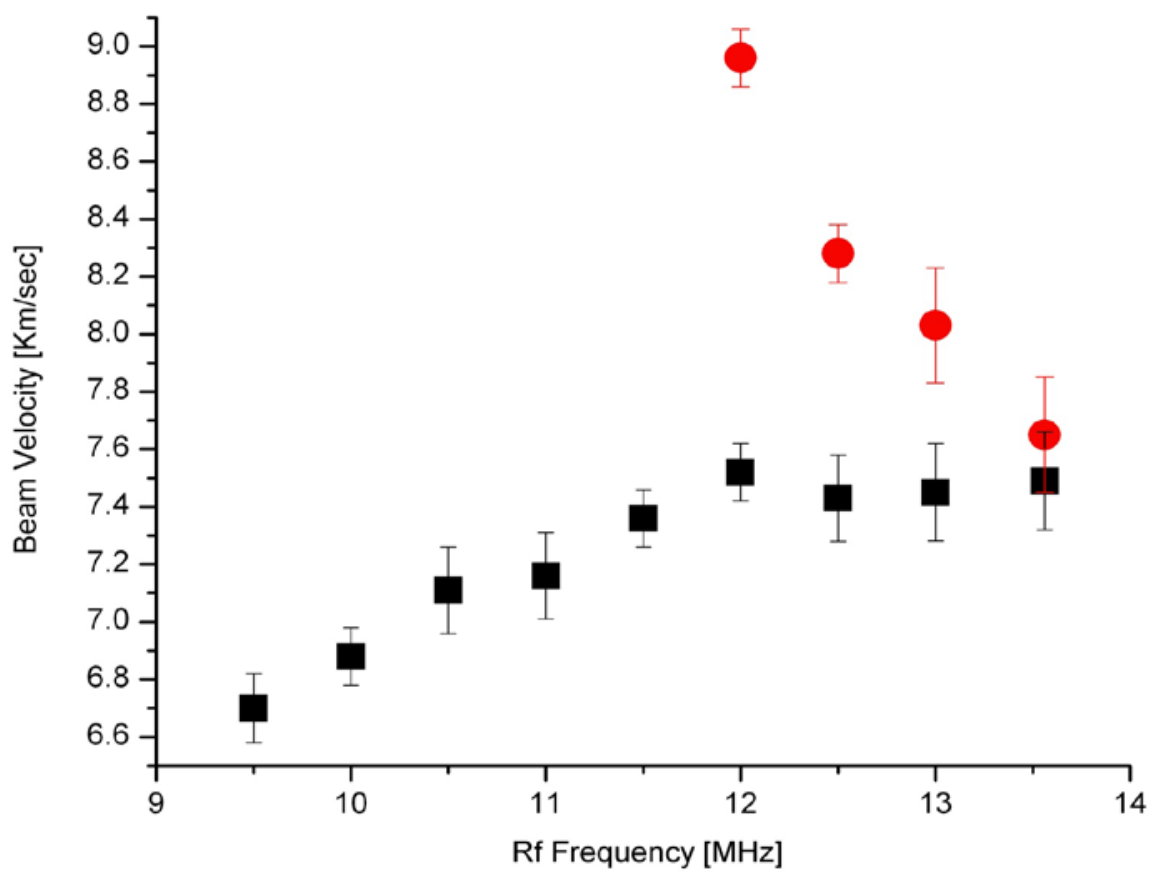

Figure 5.21. Upstream (black squares) and downstream (red circles) ion beam velocity versus antenna frequency. The velocities have been corrected for Zeeman shifts and the angle of the downstream LIF probe. The downstream ion beam vanishes for lower antenna frequencies. 
Previous experiments in our helicon source demonstrated improved power coupling into the plasma at lower antenna frequencies ${ }^{73}$ and we suggest the following interpretation of the observations reported here. At antenna frequencies below 11.5 - 12 $\mathrm{MHz}$, a stronger DL attempts to form. The accelerated ion and electron beam currents exceed some threshold and large electrostatic instabilities develop. The instabilities appear as large amplitude noise on the Langmuir probe measurements and also as "flickering” of the accelerating potential upstream of the normal double layer formation location. Because the double layer is unstable, the upstream ions are not continuously accelerated towards the expansion region and a broad ion population including an accelerated bulk population and a long tail extending down to zero drift energy results (see Fig. 5.10). Downstream of the expansion region, the ion beam does not propagate when the large instabilities are present - and is therefore not seen in the LIF data presented in Fig. 5.9 or in the RFEA data presented in Fig. 5.12. Once the rf power coupling efficiency decreases at higher antenna frequencies, a stable, but weaker, double layer forms, the electrostatic noise is significantly reduced, and the ion beam appears downstream. Consistent with this hypothesis are the measurements of the upstream bulk ion speeds and the downstream ion beam velocities (obtained from the IVDFs of Fig. 5.9 and Fig. 5.12) as shown in Fig. 5.21. The downstream ion beam velocity clearly increases with decreasing antenna frequency until the beam abruptly vanishes downstream. The upstream beam velocity is relatively constant at the higher antenna frequencies and then begins to drop at the same threshold antenna frequency for which the downstream beam vanishes. Above the threshold of $11.5 \mathrm{MHz}$, the modest increase in the strength of the DL with decreasing antenna frequency is probably related to the slight improvement in the rf coupling of the source at lower antenna frequencies as seen in previous experiments [73].

Because the appearance of the instability disrupts the DL, these measurements provide a unique means of experimentally studying the physics related to the formation of a current-free double layer in expanding plasmas. These results suggest that creation of strong DLs in expanding plasmas for plasma propulsion ${ }^{39,74}$ may be self- limited through instability growth. 


\section{Chapter 5 References}

${ }^{1}$ N. Hershkowitz, IEEE Trans. Plasma Sci. 29, 75 (2001)

${ }^{2}$ L. P. Block, Astrophys. And Space Sci. 55, 59 (1978)

${ }^{3}$ H. Alfvén, Tellus 10, 104 (1958)

${ }^{4}$ R. D. Albert and P. J. Linstrom, Science 170,1398 (1970)

${ }^{5}$ P. Carlqvist, in Proceedings of the Second Symposium on Plasma Double-layers and Related Topics, edited by Schrittweiser R and Eder G (Institute for Theoretical Physics, Innsbruck, Austria), p. 340 (1984)

${ }^{6}$ W. B. Bridges, A. N. Chester, A. S. Halsted et al., Proc. IEEE 59, 724 (1971)

${ }^{7}$ H. Hora, P. Lalousis and S. Eliezer, Phys. Rev. Lett. 53, 1650 (1984)

${ }^{8}$ J. P. Squire, F. R. Chang-Diaz, T. W. Glover, V. T. Jacobson, D. G. Chavers, R. D. Bengston, E. A. Bering, R. W. Boswell, R. H. Goulding and M. Light, Fusion Science and Technol. 43, 111 (2003)

${ }^{9}$ P. Coakley and N. Hershkowitz, Phys. Fluids 22, 1171 (1979)

${ }^{10}$ B. H. Quon and A.Y. Wong, Phys. Rev. Lett. 37, 1393 (1976)

${ }^{11}$ S. Torvén and D. Anderson, J. Phys. D. 12, 717 (1979)

${ }^{12}$ R. L. Stenzel, M. Ooyama and Y. Nakamura, Phys. Rev. Lett. 45, 86 (1980)

${ }^{13}$ M. J. Alport, S. L. Cartier and R. L. Merlino, J. Geophys. Res. 91, 1599 (1986)

${ }^{14}$ C. K. Goertz and G. Joyce, Astrophys. Space Sci. 32, 165 (1975)

${ }^{15}$ D. L. Newmann et al., Phys. Rev. Lett. 87, 255001 (2001)

${ }^{16}$ F. W. Perkins and Y. C. Sun, Phys. Rev. Lett. 46, 115 (1981)

${ }^{17}$ D. J. Trevor, N. Sadeghi, T. Nakamo, J. Derouard, R. A. Gottscho, P. D. Foo and J. M. Cook, Appl. Phys. Lett. 57, 1188 (1990)

${ }^{18}$ N. Sadeghi, T. Nakamo, D. J. Trevor and R. A. Gottscho, J. Appl. Phys. 70, 2552 (1991)

${ }^{19}$ C. Charles, R. W. Boswell, A. Bouchoule, C. Laure and P. Ranson, J. Vac. Sci. Technol. A 9, $661(1991)$

${ }^{20}$ R. Schrittwieser, I. Axnus, T. Carpenter and S. Torvén, IEEE Trans. Plasma Sci. 20, 607 (1992)

${ }^{21}$ C. Chan, M. H. Cho, N. Hershkowitz and T. Intrator, Phys. Rev. Lett. 57, 3050 (1986)

${ }^{22}$ H. Inuzuka, Y. Torri, M. Nagatsu and T. Tsukishima, Phys. Fluids 28, 703 (1985)

${ }^{23}$ Y. Takeda and K. Yamagiwa, Phys. Rev. Lett. 55, 711 (1985)

${ }^{24}$ N. Sato, R Hatakeyama, S. Iizuka, T. Mieno, K. Saeki, J. J. Rasmussen and P. Michelsen, Phys. Rev. Lett. 46, 1330 (1981) 
${ }^{25}$ S. Torvén, J. Phys. D: Appl. Phys. 15, 1943 (1982)

${ }^{26}$ R. L. Stenzel, M. Ooyama and Y. Nakamura, Phys. Rev. Lett. 45, 1498 (1980)

${ }^{27}$ N. Hershkowitz, Space Sci. Rev. 41, 351 (1985)

${ }^{28}$ P. Coakly and N. Hershkowitz, Phys. Fluids 22, 1171 (1979)

${ }^{29}$ P. Coakly and N. Hershkowitz, Phys. Lett. A 83, 1171 (1981)

${ }^{30}$ G. Hairapetian and R. L. Stenzel, Phys. Rev. Lett. 61, 1607 (1988)

${ }^{31}$ G. Hairapetian and R. L. Stenzel, Phys. Rev. Lett. 65, 175 (1990)

${ }^{32}$ G. Hairapetian and R. L. Stenzel, Phys. Fluids B 3, 899 (1991)

${ }^{33}$ R. W. Boswell, Phys. Lett. A 33, 457 (1970)

${ }^{34}$ P.A. Keiter, Experimental Investigation of Ion Temperature Anisotropy Driven Instabilities in a High Beta Plasma, Ph. D. Thesis, West Virginia University, Morgantown (1999)

${ }^{35}$ F. R. Chang-Diaz, R. H. Goulding, R. D. Bengtson, F. Wally-Baity, D. Sparks, R. G. Bussell Jr., C. G. Barber, G. McCaskill, V. T. Jacobson, M. D. carter, A. V. Ilin and T. W. Glover, Fusion Technol. 35, 243 (1999)

${ }^{36}$ J. P. Squire, F. R. Chang-Diaz, T. W. Glover, V. T. Jacobson, D. G. Chavers, R. D. Bengtson, E. A. Bering III, R. W. Boswell, R. H. Goulding and M. Light, Fusion Sci. Technol. 43, 111 (2003)

${ }^{37}$ J. T. Scheuer, K. F. Schoenberg, R. A. Gerwin, R. P. Hoyt, I. Henins, D. C. Black, R. M. Mayo and R. W. Moses Jr., IEEE Trans. Plasma Sci. 22, 1015 (1994)

${ }^{38}$ K. K. Chi, T. E. Sheridan and R. W. Boswell, Plasma Sources Sci. Technol. 8, 421 (1999)

${ }^{39}$ C. Charles and R. W. Boswell, Appl. Phys. Lett. 82, 1356 (2003)

${ }^{40}$ C. Charles, A. W. Degeling, T. E. Sheridan, J. H. Harris, M. A. Lieberman and R. W. Boswell, Phys. Plasmas 7, 5253 (2000)

${ }^{41}$ C. Charles and R. W. Boswell, Phys. Plasmas 11, 1706 (2004)

${ }^{42}$ C. Charles and R. W. Boswell, Appl. Phys. Lett. 84, 332 (2004)

${ }^{43}$ S. A. Cohen, N. S. Seifert, S. Stange, E. E. Scime, R. F. Boivin and F. Levinton, Phys. Plasmas 10, 2593 (2003)

${ }^{44}$ X. Sun, C, Biloiu, R. Hardin and E. E. Scime, Plasma Sources Sci. Technol. 13, 459 (2004)

${ }^{45}$ R. F. Boivin and E. E. Scime, Rev. Sci. Instrum. 74, 4352 (2003)

${ }^{46}$ X. Sun, A. M. Keese, C. Biloiu, E. E. Scime, A. Meige, C. Charles and R. W. Boswell, Phys. Rev. Lett. 95, 025004, (2005)

${ }^{47}$ A. Meige, R. W. Boswell, C. Charles, J.-P. Boeuf, G. Hagelaar and M. Turner, IEEE Trans. Plasma Sci. 33, 334 (2005) 
${ }^{48}$ M.A. Raadu, Physics Reports 178, 25 (1989)

${ }^{49}$ X. Sun, A Study of Ion Acceleration, Asymmetric Optical Pumping and Low Frequency Waves in Two Expanding Helicon Plasmas, Ph. D. Thesis, West Virginia University, Morgantown (2005)

${ }^{50}$ J. G. Andrews and J. E. Allen, Proc. R. Soc. London A 320, 459 (1971)

${ }^{51}$ M. A. Lieberman, C. Charles and R. W. Boswell, J. Phys. D: Appl. Phys. 393294 (2006)

${ }^{52}$ M. A. Lieberman and C. Charles, Phys. Rev. Lett. 97045003 (2006)

${ }^{53}$ A. Fruchtman, Phys. Rev. Lett. 46065002 (2006)

${ }^{54}$ D. Bohm, The characteristics of Electrical Discharges in Magnetic fields, edited by A. Guthrie and R. K. Wakerling (New York: McGraw-Hill) Chapter 3, p. 77 (1949)

${ }^{55}$ C. Chan, N. Hershkowitz and G. L. Payne, Phys. Lett. A 83, 328 (1981)

${ }^{56}$ M. A. Lieberman and A. J. Lichtenberg, Principles of Plasma Discharges and Material Processing, (Wiley, New York $2^{\text {nd }}$ ed.) p. 140-148, 691 (2005)

${ }^{57}$ O. Sutherland, C. Charles, N. Plihon and R. W. Boswell, Phys. Rev. Lett. 95, 205002 (2005)

${ }^{58}$ F. F. Chen, Phys. Plasmas 8, 3029 (2001)

${ }^{59}$ R. T. S. Chen and N. Hershkowitz, Phys. Rev. Lett. 80, 4677 (1998)

${ }^{60}$ F. F. Chen and D. D. Blackwell, Phys. Rev. Lett. 82, 2677 (1999)

${ }^{61}$ C. Charles and R. W. Boswell, Appl. Phys. Lett. 91, 201505 (2007)

${ }^{62}$ S. Chakraborty Thakur, Z. Harvey, I.A. Biloiu, A. Hansen, R. A. Hardin, W. S. Przybysz and E. E. Scime, Phys. Rev. Lett. 102, 035004 (2009)

${ }^{63}$ S. Chakraborty Thakur, A. Hansen and E. E. Scime, Plasma Sources Sci. Technol. 19, 025008 (2010)

${ }^{64}$ E. E. Scime, I. A. Biloiu, J. Carr Jr., S. Chakraborty Thakur, M. Galante, A. Hansen, S. Housmandyar, A. M. Keesee, D. McCarren, S. Sears, C. Biloiu and X. Sun, Phys. Plasmas 17, 055701 (2010)

${ }^{65}$ Z. Harvey, S. Chakraborty Thakur, A. Hansen, R. A. Hardin, W. S. Przybysz and E. E. Scime, Rev. Sci. Instrum. 79, 10F314 (2008)

${ }^{66}$ R. A. Stern and J. A. Johnson III, Phys. Rev. Lett. 34, 1548 (1975)

${ }^{67}$ I. D. Sudit and F. F. Chen Plasma Sources Sci. Technol. 3,162 (1994)

${ }^{68}$ I. A. Biloiu, E. E. Scime, C. Biloiu and S. Cohen, Proceedings of the XXVIII International Conference on the Physics of Ionized Gasses, Prague, 1537 (2007)

${ }^{69}$ I. H. Hutchinson, Principles of Plasma Diagnostics (Cambridge University Press, Cambridge 1987) 
${ }^{70}$ A. Aanesland, C. Charles, M. A. Lieberman and R. W. Boswell, Phys. Rev. Lett. 97, 075003 (2006)

${ }^{71}$ S. P. Gary and N. Omidi, J. Plasma Phys. 37, 45 (1987)

${ }^{72}$ D. A. Gurnett and A. Bhattacharjee, Introduction to Plasma Physics, (Cambridge University Press, 2005)

${ }^{73}$ P.A. Keiter, E.E. Scime and M. M. Balkey, Phys. Plasmas 4, 2741 (1997)

${ }^{74}$ F. R. Chang-Diaz, Sci. Am. 283, 90 (2000) 


\section{CHAPTER 6: CAVITY RING DOWN SPECTROSCOPY AS A TOOL TO MEASURE VDF}

\subsection{Introduction to Cavity Ring Down Spectroscopy}

Cavity Ring Down Spectroscopy [CRDS] is an alternative way of making ultra sensitive absorption measurements. Absorption spectroscopy measures the loss of electromagnetic energy after the radiation passes through a cell containing the sample under study. Atoms, ions or molecules absorb radiation at a wavelength, $\lambda_{i k}$, which excite them from a lower energy state, $E_{i}$, to an upper energy state $E_{k}$, according to

$$
\lambda_{i k}=\frac{h c}{\left(E_{k}-E_{i}\right)},
$$

where $c$ in the speed of light and $h$ is the Plank's constant. Consequently, radiation at this wavelength is attenuated at the exit of the sample cell. If the energy states are both bound levels, the transmitted spectrum consist of absorption discrete lines. Because atoms and molecules have a unique and identifiable set of energy levels, a measurement of the attenuated wavelengths allows identification of the species present. According to the Beer-Lambert law, the transmitted intensity, $I_{\text {trans }}(\lambda)$, at the wavelength $\lambda$ is related to the incident intensity $I_{0}(\lambda)$ by

$$
I_{\text {trans }}(\lambda)=I_{0}(\lambda) e^{-\alpha(\lambda) \square_{\text {eff }}} \text {, }
$$

where $\alpha(\lambda)$ is the absorption coefficient (which is a product of the absorption crosssection $[\sigma(\lambda)]$ of that line and the density $[N]$ of the corresponding species) and $l_{\text {eff }}$ is the effective path-length for the absorption. For weak absorption, the first order Taylor expansion of $\exp \left(-\alpha(\lambda) l_{\text {eff }}\right)$ is equal to $\left(1-\alpha(\lambda) l_{\text {eff }}\right)$, so that the absorption coefficient can be calculated from

$$
\alpha(\lambda)=\frac{I_{0}(\lambda)-I_{\text {trans }}(\lambda)}{I_{0}(\lambda) \cdot l_{\text {eff }}}
$$


The minimum detectable concentration of absorbers for a specific absorption transition is inversely proportional to the effective sample path-length and directly proportional to the minimum intensity fluctuation of the system.

Traditional absorption measurements involve the determination of two parameters: the incident power of a radiant beam on a sample $\left(P_{o}\right)$ and the radiant power of the beam after it has passed through the sample $\left(P_{\text {trans }}\right)$. As the concentration of the absorbing species decreases, the value of $P_{\text {trans }}$ approaches $P_{o}$. The detection limit is reached when the magnitudes of the two beams become statistically indistinguishable. By contrast, CRDS monitors the exponential decay of radiation in a resonant optical cavity. For a given wavelength, the time associated with this exponential decay is related to the absorption coefficient of the sample contained within the cavity. Improvements in the detection limit achieved with CRDS can be of the order of $10^{4}$ or more compared to traditional absorption methods.

\subsubsection{Historical background}

The fundamental concept behind the development of Cavity Ring Down Spectroscopy [CRDS] technique can be traced back to the experimental efforts in the early 1980s which were aimed at characterizing the reflectivity of Highly Reflective [HR] mirror surfaces for several different applications. As HR mirrors became more important for various applications like high power lasers, gyroscopes, optical frequency standards etc., the need to be able to measure the reflectivity of these mirrors grew. The problem confronting further development was the lack of precision in the characterization of mirror coatings; typical uncertainties in measured mirror reflectivities were of order $0.1 \%$. Without more precise measurements, incremental improvements in coating quality were problematic.

Two competing techniques were developed to measure mirror reflectivity based on the decay constant of a linear cavity Fabry-Perot resonator formed by two planoconcave mirrors: one was by measuring the phase shift accrued by amplitude-modulated light as it traversed the resonator ${ }^{1,2,3}$ and the other was by measuring the light intensity decay constant when the excitation source was abruptly interrupted. ${ }^{4,5}$ In 1980 , Herbelin et. $a{ }^{2}{ }^{2}$ demonstrated that the phase shift method (Fig. 6.1.1a) could accurately measure 
the averaged reflectivity of a pair of mirrors in a cavity to $0.01 \%$. This was followed by the work of Anderson et. al. in 1984, ${ }^{4}$ which utilized a shuttered laser injection into an optical cavity (Fig. 6.1.1 b) and measured the reflectivity of a pair of mirrors to an accuracy of $0.0005 \%$.

In the experiment by Anderson et. al. ${ }^{4}$, the output of a CW He-Ne laser was directed to the input of a linear optical resonator consisting of two plano-convex lenses facing each other. Before the resonator, the laser passed through a Pockel's cell and mode matching lenses. When the laser output frequency accidentally coincided with a sample cavity mode frequency, energy was efficiently coupled into the cavity. This was accompanied by an increased transmission out of the cavity in the forward direction. The transmitted intensity was monitored with a photo-detector. When the transmitted intensity sharply increased, the laser light was then terminated by the Pockel's cell and the decay of the light inside the cavity recorded. The reflectivity of the mirrors could then be calculated from the decay lifetime.

This technique was improved by Rempe et. al. ${ }^{5}$ in 1992 to measure the reflectivity of HR mirrors. They mounted one of the super-reflectivity mirrors onto a piezoelectric actuator so that the cavity length could be scanned across the test laser linewidth to intentionally create a resonance. When a sufficient light level was obtained, the laser light was deflected from the cavity using an acousto-optic modulator. The decay of the light intensity from the cavity was recorded and numerically fit.

Anderson's time domain approach was adopted by super-mirror manufacturers and was commercialized under the name of "cavity loss-meters". All measurements were performed at a single wavelength and the technique was not applied to spectroscopy. Interestingly, Anderson ${ }^{4}$ remarked: "the cavity optical path was evacuated: we have made tests in open air cavities but found our results depended on the ambient Pasadena air quality." Anderson et. al. ${ }^{4}$ had measured molecular absorption losses in atmosphere, in serendipity, without realizing that this method could be used as a spectroscopic tool.

It was not until 1988, that this method was used by O'Keefe and Deacon, ${ }^{6}$ with a nitrogen-gas-pumped pulsed (15 ns) dye laser emitting at $690 \mathrm{~nm}$, to investigate transitions of molecular oxygen. This was the first example of pulsed-cavity ring down spectroscopy [P-CRDS]. Another early application ${ }^{7}$ of P-CRDS appeared in 1990 when 
the UC Berkley group used a nitrogen-gas-pumped dye laser (450 nm, $15 \mathrm{ns)}$ to investigate the spectroscopic constants of jet-cooled metal clusters in a molecular beam. Using excimer pumped dye laser emitting in the ultraviolet (365 nm for silver, 405-413 $\mathrm{nm}$ for aluminum and $400 \mathrm{~nm}$ for copper), P-CRDS was further extended beyond visible spectroscopy to investigate the vibronic isotope shifts and rotational bands of cluster spectra. ${ }^{8}$ Since then, P-CRDS has been extended to the mid-infrared (using a Raman shifter cell) to study spectra of stable organic molecules and water clusters. ${ }^{9}$

Throughout the 1990s, applications of P-CRDS were extended to detailed spectroscopic studies of line intensities, transition wavelengths and coupling effects in vibrational and rotational spectra in gas phase species by Lehmann and Romanini at Princeton University. ${ }^{10}$ The Meijer group at the University of Nijmegen, Netherlands, is another source of innovations in P-CRDS techniques. They introduced the concept of using short cavities having a dense transverse mode structure, and collecting all the modes to avoid mode beating, to improve the resolution of P-CRDS. ${ }^{11}$ Their group was the first to apply P-CRDS to the study of laser-desorbed, jet-cooled organics (diphenylamine $)^{12}$ and to demonstrate that this method can be used in the UV for trace gas detection. They achieved P-CRDS sensitivities of ten parts per billion for ammonia and one part per trillion for elemental mercury in air. ${ }^{13}$ They were the first to identify the laser bandwidth effects in P-CRDS that result in multi-exponential decays when the laser linewidth is broader than the absorption feature. Since the mid 1990s, the Zare group at Stanford University has been applying P-CRDS in the ultraviolet $(216 \mathrm{~nm})$ for the detection of methyl radicals in a hot filament reactor used for chemical vapor deposition of diamond films. They demonstrated that P-CRDS is a much more sensitive technique than multi-pass direct absorption spectroscopy and that it is possible to measure spatially resolved concentration profiles (along the axis perpendicular to the ring down cavity). They were the first to show that multimode cavity excitation results in mode effects that severely distort the ring down decay. ${ }^{14}$

At the National Institute of Standards and Technologies (NIST), in Maryland, Hodges, Looney and van Zee were the first to construct dedicated experiments for the study of the physics of P-CRDS. They demonstrated the limitations of using laser sources that are broadband with respect to the absorption features. ${ }^{15}$ They studied how these 
limitations could be overcome by using cavities where only a single mode is excited, so that the resolution of the P-CRDS experiment is due to the linewidth of the ring down cavity and not that of the laser. They also studied the effects of mode beating and multi mode excitation on noise in the ring down curves and the subsequent reduction in detection sensitivity. ${ }^{16}$ Over the years, P-CRDS has become increasingly popular amongst spectroscopists as a very sensitive tool from the deep ultraviolet to the far infrared, with applications ranging from reaction studies in hostile environments like flames ${ }^{17}$ and hollow cathode discharges ${ }^{18}$ to detailed spectroscopic studies of complex molecules.

The use of pulsed lasers made the experimental requirements of CRDS easily achievable, but also imposed severe limitations. Pulsed lasers are not only bulky and expensive; they have a larger linewidth, so unless care is taken to mode match the laser to one single mode of the ring down cavity, P-CRDS can have problems of multimode excitation and is limited in resolution by the laser linewidth. To overcome these problems, Lehmann proposed the use of continuous wave (CW) laser sources. Singlemode, CW lasers that are modulated externally have a higher repetition rate, limited only by the buildup time of light intensity in the ring down cavity, and also have narrower line widths. The resulting increased overlap between the laser and the ring down cavity linewidth results in energy buildup inside the ring down cavity. Furthermore the ability of using CW laser sources enables the use of the rather inexpensive diode laser sources. Initial efforts to use a CW diode laser in CRDS focused on optically locking a laser diode to a high finesse cavity. ${ }^{19}$ This approach was not entirely successful because of the lack of long term stability, due to thermal drift, in locking the laser to the same cavity mode. It was not until 1997, that the experimental method using a continuous wave laser, developed by Rempe et. al. ${ }^{5}$ to measure reflectivity was adapted by Romanini et. al. ${ }^{20}$ as a spectroscopic tool and henceforth came to be commonly known as CW-CRDS. They avoided the optical locking problem by sweeping one resonant mode of their ring down cavity through the CW laser bandwidth. This ensured light intensity buildup inside the cavity. When the light intensity in the cavity was sufficiently strong and detected by a photo receiver, the input laser beam was deflected by an acousto-optic-modulator (AOM), and the ring down decay was recorded. Extensive mode matching was carried 
out to ensure that only the $\mathrm{TEM}_{00}$ modes (see Section 6.2.1) could be excited in the cavity. The use of cw-diode lasers made the experiments less expensive and increased the resolution by another couple orders of magnitude by ensuring single mode excitation of the cavity. The downside of this approach is a much more complicated experimental apparatus. It is important to note than even though this method came to be known as CWCRDS, it is not really a continuous method. Over the last decade CW-CRDS has found many applications, including plasma spectroscopy.

For the reader interested in going beyond this brief history of CRDS, there are some excellent reviews on CRDS that include specific details of different aspects of this spectroscopic method and its adaptations. ${ }^{2122,23,24,25,26,27}$

\subsubsection{Basic principles}

In traditional absorption spectroscopy, if the analyte is the only absorption species in the path of radiation at a particular wavelength, its concentration can be determined by means of the Beer-Lambert law:

$$
I_{t}=I_{o} e^{(-\sigma L N)},
$$

where $I_{t}$ is the transmitted intensity, $I_{0}$ is the incident intensity, $\sigma$ is the absorption cross section for the species at the wavelength of measurement in $\mathrm{cm}^{2}, L$ is the path length of the incident light in the absorbing medium in $\mathrm{cm}$ and $N$ is the number density of the absorbing species in $\mathrm{cm}^{-3}$. It is clear that if the absorption feature is very weak, then the incident and the transmitted intensities are approximately equal to each other. The problem then lies in the accurate measurement of a very small difference between two large numbers, both of which are susceptible to the uncertainties of experiments. Moreover if the light source used for the absorption measurements is a pulsed laser, then the shot to shot variation in the incident intensity can easily mask an absorption feature. The CRDS technique is based upon the measurement of the rate of decay, rather than the magnitude of absorption, of a light pulse confined in a closed optical cavity. The advantage over normal absorption spectroscopy results from the intrinsic insensitivity to 
light source intensity fluctuations and the extremely long effective path lengths that can be realized in stable optical cavities (see Section 6.2.1).

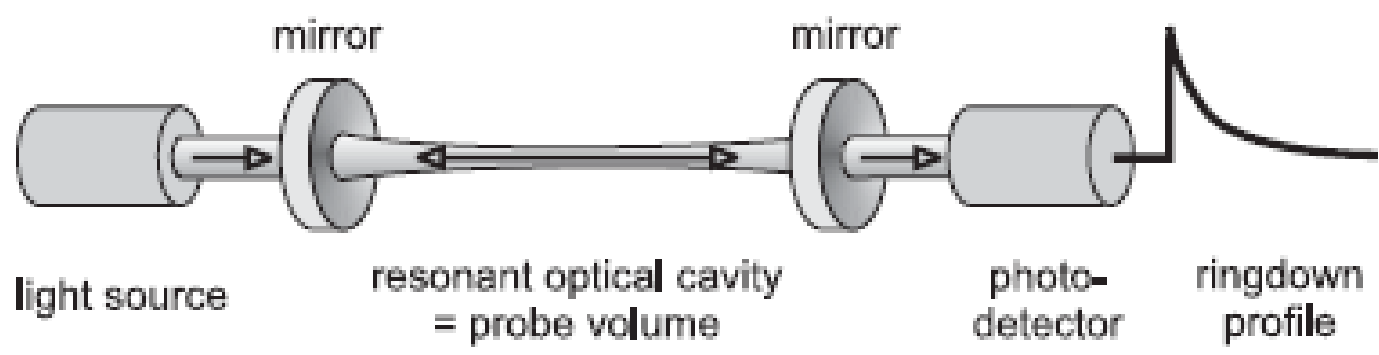

Figure 6.1. Schematic set-up for a typical pulsed cavity ring down experiment. Figure obtained from Ref. [26].

Because of its historical importance and intrinsic simplicity, we shall review the "photon bullet" model of CRDS in this section. We shall revisit this picture in Section 6.2.1 and see the limitations of this model and also introduction the necessary formulation to understand mode formation in cavities, without which it is not possible to explain CW-CRDS. The P-CRDS is most easily illustrated with the cartoon describing the experimental set-up shown in Fig 6.1. The light of a pulsed laser is injected into a stable optical cavity formed by two highly reflective plano-concave mirrors. The light pulse is repeatedly reflected back and forth between the two mirrors and the small amount of light lost due to residual mirror transmission with each reflection is monitored by a photo detector placed behind the exit mirror. In this simple picture, when the duration of the laser pulse width is shorter than the round trip time of the pulse in the cavity, the detector will see a train of pulses, each with less intensity than the previous one, as schematically shown in Fig. 6.2. 


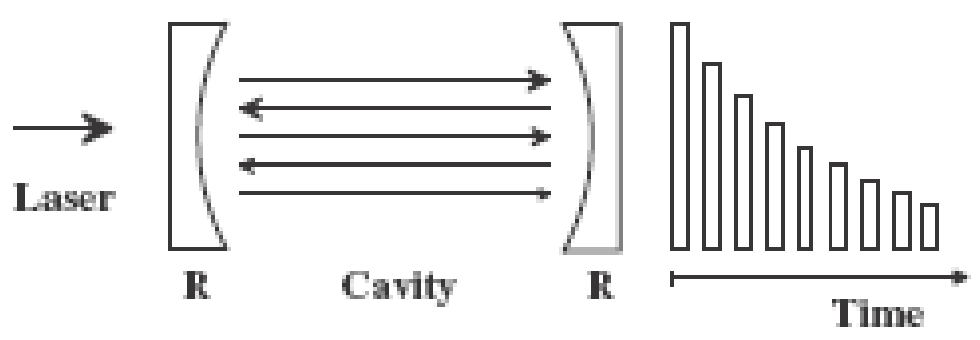

Figure 6.2. Schematic description of the "Photon bullet" model of pulsed cavity ring down spectroscopy technique. Figure obtained from Ref. [27].

The separation between the adjacent pulses is equal to the round trip time $\left(t_{r}\right)$ in the cavity $t_{r}=2 L / c$; where $L$ is the length of the cavity and $c$ is the speed of light. Even in an empty cavity, the intensity of the pulses will decrease due to the losses at the mirrors. The presence of an absorber in the cavity further increases the loss rate. If the reflectivity of the mirrors is $R$ and the transmission is given by $T$, for ideal mirrors $T=1-R$. After one pass through the cavity, the intensity of the first optical pulse at the detector is obtained from Beer-Lambert's law:

$$
I_{0}=I_{\text {laser }} T^{2} e^{(-\alpha d)}
$$

where $\alpha$ is the frequency dependant absorption coefficient of the medium in the cavity. It is assumed that the absorbing sample is present only over a length $d$ in the cavity. The intensity of the second pulse is then given by:

$$
I_{1}=I_{0} R^{2} e^{(-2 \alpha d)},
$$

where $R^{2} \exp (-2 \alpha d)$ accounts for the loss due to one additional complete round trip in the cavity. Hence, after $n$ complete round trips, the cavity intensity is

$$
I_{n}=I_{0}\left[R e^{(-\alpha d)}\right]^{2 n}=I_{0} R^{2 n} e^{(-2 n \alpha d)} .
$$


Since the loss per pass is very small, typical electronics have enough bandwidth that even though the individual pulses circulating in the cavity are not seen, the overall cavity decay is easily measured. Thus, the discrete number of round trips $n$ can be replaced by the continuous parameter time, $t=n \times 2 L / c$. Moreover, $R^{2 n}$ can be rewritten as $\exp (2 n \ln R)$, which gives:

$$
I(t)=I_{0} e^{\left(\frac{t c}{L}(\ln R-\alpha d)\right)} .
$$

Again, since the mirrors are highly reflective (generally referred to as super mirrors), $R \sim 1$, and therefore $\ln R \sim$ - (1 - R) and the above formulae becomes

$$
I(t)=I_{0} e^{\left(-\frac{t c}{L}(1-R+\alpha d)\right)} .
$$

The ring down time $\tau$ is defined as the time required for the exponentially decaying light intensity to fall to $1 / e$ of the initial intensity, by:

$$
\tau=\frac{L}{c(1-R+\alpha d)} .
$$

In general, light scattering and light absorbing effects should be included. Moreover, the reflectivity of the super mirrors depends on the frequency as well. Therefore, a more general formula is given by:

$$
\tau(v)=\frac{L}{c\left[1-R(v)+\Sigma_{i} \sigma_{i}(v) \int_{0}^{d} N_{i}(x) d x\right]},
$$

where the sum is over all species $i$ with frequency dependant absorption or scattering cross sections and the integral reminds us that the number density is line integrated. Typically, the frequency interval chosen is narrow so that the frequency dependence of 
the scattering cross sections and the mirror reflectivity can be neglected. In that case, an effective reflectivity $R_{\text {eff }}$ is defined which includes all broadband absorption and scattering losses in addition to the mirror loss.

In a typical CRDS experiment, the cavity ring down time is measured as a function of the laser frequency. The maximum change in the ring down time, with respect to the case when there is no absorber in the cavity, will be when the laser frequency is tuned to the peak of the absorption feature. The absorption spectrum is obtained by plotting the cavity decay rate $k=1 / \tau$ (or the cavity loss $1 / c \tau$ ) as a function of frequency,

$$
\frac{1}{c \tau(v)}=\frac{(1-R)}{L}+\alpha(v) \frac{d}{L} .
$$

The cavity loss is then the effective sum of two terms; first is the mirror loss which determines the baseline of a typical CRD spectrum and the second is the term due to the absorption. The off resonance mirror loss is the effective loss without the presence of any absorbers in the cavity and is given by:

$$
\tau_{0}=\frac{L}{c(1-R)}=\frac{1}{k_{0}} .
$$

Hence the absorption due to the sample in the cavity is effectively given by the difference between the off-resonance and the on-resonance decay time constants.

$$
\alpha(v) \frac{d}{L}=\frac{k-k_{0}}{c}=\frac{1}{c}\left(\frac{1}{\tau}-\frac{1}{\tau_{o}}\right) .
$$

If the absorption feature is large where the effective reflectivity changes significantly, then the cavity decay rate can be measured with and without the sample to determine the absorption coefficient. 


\subsubsection{Sensitivity of CRDS}

The limiting sensitivity of a CRD instrument is the minimum possible absorption coefficient that can be detected by the spectrometer as the ring down times on and off resonance (or with and without absorber in the cavity) come closer to each other. The minimum detectable absorption possible with this technique is

$$
\left[\alpha(v) \frac{d}{L}\right]_{\min }=\frac{\left(k-k_{0}\right)_{\min }}{c}=\frac{\Delta k_{\min }}{c}=\frac{1}{c \tau_{0}^{2}} \Delta \tau_{\min }=\frac{1-R}{L}\left(\frac{\Delta \tau}{\tau_{0}}\right)_{\min } .
$$

The smallest absorption coefficient measurable is thus proportional to the smallest measureable change in the ring down time for a given cavity. It is also obvious that higher reflectivity mirrors make the experiment more sensitive. While it appears that longer cavities would increase sensitivity, this is true only up to a certain limit. The fraction of light that passes through a cavity usually decreases, with only a small increase in the ring down time, once the sample loss (including scattering) exceeds the mirror losses. For atmospheric P-CRDS, Rayleigh scattering by a gaseous sample is a significant loss. It is generally accepted that in the above equation $R$ is in fact an effective value of the reflectivity $\left(R_{\text {eff }}\right)$ of the mirrors, taking into account all other loss mechanisms such as broadband scattering, and is usually smaller than the quoted mirror reflectivity.

Thus, the effective sensitivity of CRDS is limited by the accuracy of the determination of the ring down times. This is typically achieved by using digitizers with a very high vertical resolution (10 bits of memory or higher) and a very large horizontal resolution (sample rates of $20 \mathrm{Ms} / \mathrm{sec}$ or faster) to accurately record the ring down. A non-linear fit to the measured intensity curve is used to extract the time constant for the exponential decay. In practice the measurement accuracy is limited by fluctuations in the shape of the ring down. Often the rings down curves are multi-exponential due to the multimode excitation of the ring down cavity. Only single mode excitation ensures a clear exponential decay with a unique time constant. In most of the reported CRDS experiments, fluctuations in the value of the ring down time are of the order of $1 \%$.

Equation 6.15 clearly shows the advantage of the CRDS technique. To achieve a very high sensitivity in measuring the absorption coefficient, only a moderate accuracy in 
measuring the time constants is required. For example, with a $10 \mathrm{~cm}$ long cavity and 99.99\% reflectivity mirrors, only 1\% accuracy in the determination of the ring down time constant gives a minimum detectable absorption of $10^{-7} \mathrm{~cm}^{-1}$. The effective absorption path length for such an experiment is one kilometer!

The fact that CRDS is practically independent of shot-to-shot intensity fluctuations of the source laser is also critically important. Effectively, the intensity of the same pulse is being compared after every round trip. Another attractive feature of CRDS is that it is independent of absorption occurring along the optical path outside the mirror cavity, as long as that external absorption does not attenuate the signal beyond the detection level. As an example, trace moisture concentrations at parts per trillion have been detected, even though the air through which the laser beam traveled had moisture concentrations many orders of magnitude higher. ${ }^{28}$

In summary, P-CRDS is typically sensitive to absorption coefficients as small as $10^{-7} \mathrm{~cm}^{-1}$. Single mode excitation can help increase the sensitivity, but such experiments are more technically challenging due to need to lock the laser to the ring down cavity and simultaneously scan the laser wavelength.

\subsubsection{Conventional CRDS experimental apparatus}

The popularity of P-CRDS comes from not only the achievable sensitivity (this is rivaled by other indirect techniques such as Laser Induced Fluorescence [LIF], ${ }^{29,30}$ Resonance-Enhanced Multi-Photon Ionization [REMPI], ${ }^{31}$ or Intra-Cavity Laser Absorption Spectroscopy $\left[\mathrm{ICLAS}^{32}\right.$ ), but also because of the simplicity of the experimental apparatus. Moreover, P-CRDS is a direct absorption technique without the need of any reference cells. Intrinsically there are no limitations to the spectral region for the application of P-CRDS, as long as mirrors with sufficient high reflectivity, detectors with enough gain and fast response, and tunable pulsed lasers at the needed wavelengths are available. Fig. 6.3 is a schematic representation of the experimental apparatus most commonly used for P-CRDS.

The essential P-CRDS apparatus consists of a tunable pulsed laser, a ring down cavity made from two highly reflective mirrors, a fast (for temporal response) detector, a high-resolution (for accuracy in measuring the amplitude) analog-to-digital converter, 
and a computer for data processing. The length of the cavity and the radius of curvature of the mirrors should be chosen such that the mirrors form an optically stable cavity. If the distance between the mirrors is $L$ and the radius of curvature of the two identical mirrors are $r$, then the cavity is optically stable if $0<L<r$ or $r<L<2 r$ (see Section 6.2.1 for additional details). The mirrors must be placed in adjustable mounts so that the cavity can be properly aligned. In some cases, to increase the accuracy, the use of mode matching optics (typically steering mirrors and/or a spatial filter) between the laser source and the cavity is necessary. After the exit mirror, one can also use a filter and a lens combination to focus the emitted light intensity onto the detector.

There are some extensive reviews on minor variations of this general experimental configuration. ${ }^{21,22,23,24,25}$

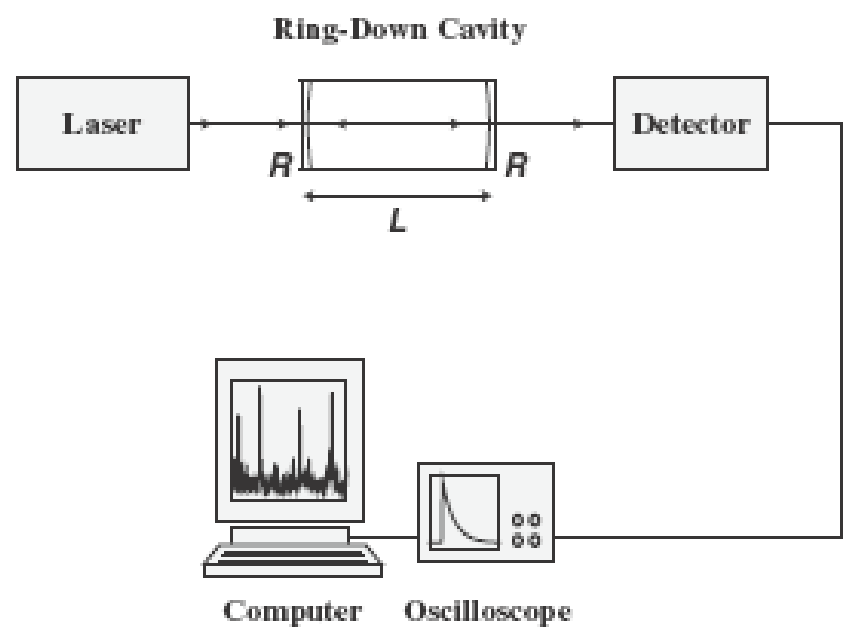

Figure 6.3. Schematic of a typical P-CRDS experiment. Figure obtained from Ref. [27].

\subsection{Continuous wave cavity ring down spectroscopy [CW-CRDS]}

Excitation of a single longitudinal mode of the ring down cavity provides the best sensitivity. Typically pulsed lasers have a very broad linewidth and the only way to obtain single mode excitation is to use a Fourier transform limited pulsed laser (linewidth $\sim 100 \mathrm{MHz}$ ) in conjunction with a very short cavity. Typical Doppler or subDoppler measurements with these systems are very challenging in practice. On the other hand, cw-lasers typically have a narrow bandwidth of a few $\mathrm{MHz}$ and are a natural choice 
for such experiments. With a CW laser, even longer cavities can be used; resulting in longer ring down times and improved accuracy. Moreover, in contrast to pulsed lasers, cw lasers, especially diode lasers, are relatively cheap, compact, easily tunable, and portable. As noted previously, $\mathrm{n}$ the literature, CRDS using $\mathrm{cw}$ lasers as the light source is termed as CW-CRDS.

In at the initial discussion of the basic principles of CRDS presented here, we understood the CRDS process through the intuitive picture of laser pulses bouncing back and forth incoherently between the two cavity mirrors. This picture captures the essence of CRDS and explains the ring down nature of the decay, but is an over-simplification and cannot explain certain other important features of CRDS. It ignores the effect of interference effects in the cavity and thus cannot explain why the cavity typically admits, from the relatively broad bandwidth of the input pulse, only very narrow (few $\mathrm{kHz}$ in width) resonant modes. We note that is has been experimentally demonstrated that CRDS is only responsive to sample absorption at the resonant frequencies of the excited cavity modes of the cavity. This is because a CRDS cell is in fact an etalon with very high finesse.

Since the "photon bullet" model of CRDS ignores the electromagnetic wave nature of the laser, this description is completely inappropriate of $\mathrm{cw}$ lasers are used as the light source for exciting the cavity. To understand CW-CRDS, we first review the properties of high-finesse optical cavities. Then we consider the experimental challenges that arise in implementing CW-CRDS and how they can be overcome in practice.

\subsubsection{Properties of Optical Cavities}

Before getting into the detailed properties of optical cavities, it is helpful to understand what is meant by a resonance in an optical cavity. In any physical system, an LC circuit for example, a resonance occurs when the frequency of a periodic driving force approaches a natural oscillation of the system. In the LC circuit, the electromagnetic energy stored in the circuit is periodically converted from an electric field stored by a capacitor to a magnetic field stored by the inductor. This is a typical example of what is called a "lumped" resonator since the energy is stored in physically 
distinctly different parts of a system. In a similar sense, a cavity resonator also stores energy that oscillates between one form and another. But in contrast to a "lumped" resonator, these are classified as "distributed" resonators, where the various forms of energy are distributed throughout the physical extent of the cavity. The energy is stored in an electromagnetic cavity in the form of oscillating electric and magnetic fields. Again, contrasting to "lumped resonators" where there is only one resonant frequency, cavity resonators can support a large number of different electromagnetic oscillations.

For the microwave region of the electromagnetic spectrum, resonant cavities consist of closed hollow conducting material of various shapes. For the optical region of the electromagnetic spectrum, due to the length scales involved, it is impractical to use hollow conductors (the required cavity size is too small). It is more practical to form optical cavities by proper arrangement of two or more mirrors. The simplest cavity can thus be formed by two mirrors facing each other. By eliminating the side walls of a hollow conducting cavity, a large number of unwanted modes are eliminated.

On the basis of cavity losses, an optical cavity can be classified into two broad categories: stable or unstable. An optical cavity is said to be geometrically stable when a paraxial ray of light is refocused into the cavity even after a large number of successive reflections from the mirrors, so that the electromagnetic energy is contained or trapped within the cavity. If the ray escapes the cavity (commonly known as "to walk away") after some finite number of reflections, then the cavity is said to be geometrically unstable.

Optical cavities formed from only two mirrors are known as "linear" or "standing wave" cavities. There can also be optical cavities consisting of three or more mirrors arranged in a circular pattern and such cavities are known as "ring” or "travelling wave" cavities. Here we discuss only linear cavities as they are most commonly used for CRDS.

While the stability of a cavity is based on geometrical optics, to understand mode formations in a cavity, one must take into account the full wave nature of electromagnetic radiation. Standing waves that develop within the cavity are known as the cavity modes. In linear cavities under paraxial conditions (when the energy is confined near the optical axis) a mode develops when the electric field of the oscillating light wave vanishes at the boundaries of the cavity. 
Most of the general properties of an optical cavity are based on the studies of a typical Fabry-Perot interferometer, which is a set of two plane and perfectly parallel reflecting mirrors, each with reflectivity $R$, facing each other. Due to multiple reflections and transmissions, an incident beam is divided into an infinite number of parallel rays, such that the interference fringes are formed at infinity. If the angle of the incident coherent light beam is zero, the Fabry-Perot interferometer effectively becomes a perfect optical resonator. The transmission of light from such a resonator is given by an Airy function which is shown in Figure 6.4 and is commonly known as a "frequency comb".

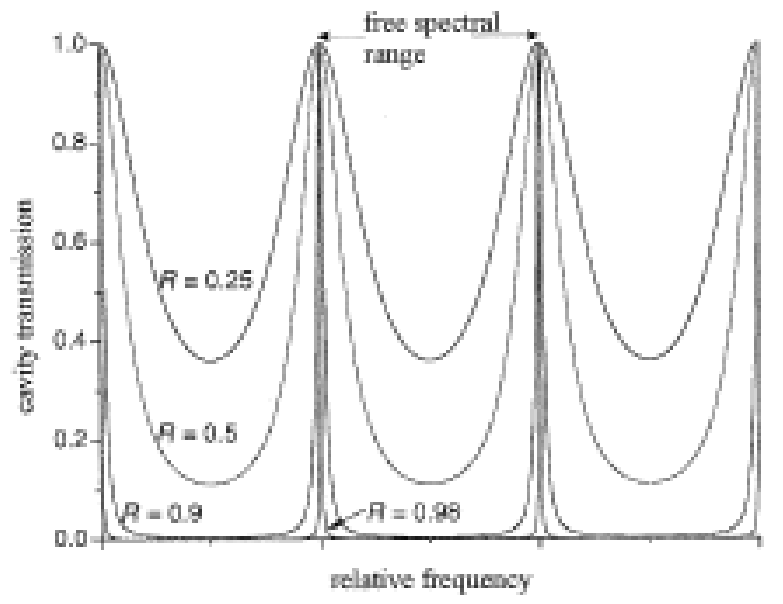

Figure 6.4. Cavity transmission comb: typical light intensity transmitted by a FabryPerot cavity. Figure obtained from Ref. [27].

These equally spaced maxima, in frequency space, in the transmission of a cavity are the "modes". In an optical cavity, light fits inside the cavity for only certain electromagnetic frequencies. Optical cavities are characterized by several parameters and those that are most relevant for CRDS experiments are free spectral range, cavity finesse, optical stability and g-parameters, cavity mode structure, cavity spot size, and mode matching.

The free spectral range (FSR) is the frequency separation (longitudinal mode spacing) between two adjacent maxima (longitudinal modes) in the transmission of an optical cavity. For a linear cavity of length $L$, the FSR is 


$$
\Delta v_{F S R}=\frac{C}{2 n_{r} L}
$$

where $c$ is the speed of light and $n_{r}$ is the refractive index of the medium inside the cavity. For a typical $100 \mathrm{~cm}$ long cavity the FSR is $150 \mathrm{MHz}$, which is much smaller than the bandwidth of most pulsed dye lasers and optical parametric oscillators ( $3 \mathrm{GHz}$ ). Thus, most pulsed lasers can be tuned continuously in a CRDS experiment because the laser pulse can overlap with 20 or more cavity modes regardless of the central frequency of the laser. On the other hand, most cw lasers have a bandwidth of a few $\mathrm{MHz}$, which is much smaller than the FSR of the cavity. So mode matching to the cavity is required. Extreme care has to be taken to tune a cw laser through a cavity mode and this process introduces significant complexity in a CW-CRDS experiment.

The finesse of an optical cavity is an effective measure of the width (FWHM) of the cavity modes and also determines the maximum possible circulating power inside the cavity. The finesse $(F)$ of a linear stable cavity is

$$
F=\frac{\pi \sqrt{R}}{1-R}
$$

If the mirrors of a $100 \mathrm{~cm}$ long cavity are $99.99 \%$ reflective, the finesse is 31500 and the FWHM is only $\sim 4.8 \mathrm{kHz}$. Since only light of frequencies resonant with the cavity modes can couple into the cavity, optimum coupling is achieved with a narrow bandwidth lasers.

As noted previously, the cavity must be optically stable, to confine the laser beam after successive reflections between the two mirrors. The stability conditions for an optical cavity constructed from two concave spherical mirrors $\left(\mathrm{M}_{1}\right.$ and $\left.\mathrm{M}_{2}\right)$ with radius of curvature $r_{1}$ and $r_{2}$ (and hence focal lengths of $f_{1}=r_{1} / 2$ and $f_{2}=r_{2} / 2$ ) separated by a distance $L$ can be obtained from geometrical optics and are ${ }^{33}$

$$
0<g_{1} g_{2}<1 \text {, where } g_{i}=1-\frac{L}{r_{i}} \text {. }
$$


For an optical cavity consisting to two identical mirrors $\left(r_{1}=r_{2}=r\right)$ the cavity is optically stable if $0<L<r$ or $r<L<2 r$. Typically, if the mirrors are separated by a distance less than twice their radius of curvature, then the cavity is optically stable (experimentally it is very difficult to achieve confocal geometry, i.e. $r=L$ ). Certain configurations like the symmetric confocal $\left(r_{1}=r_{2}=L\right)$, concentric $\left(r_{1}=r_{2}=L / 2\right)$ and plane parallel ( $r_{1}=r_{2}=$ infinity) lie on the edge of stability, as seen from the stability diagram shown in Figure 6.5 where $g_{1}$ is plotted against $g_{2}$. On this diagram, the points on the shaded region represent stable cavities, capable of trapping injected beams. Points that lie outside the shaded region correspond to unstable periodic focusing cavities, where the rays steadily migrate upon successive reflections until they finally exit the cavity.

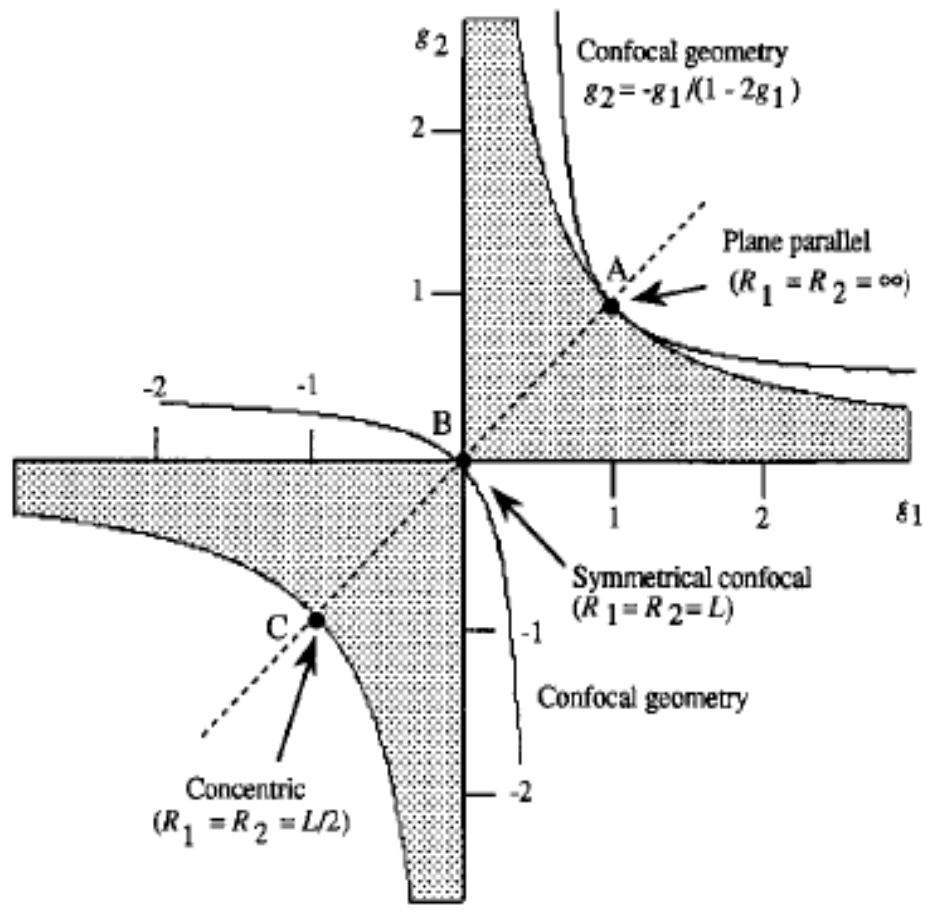

Figure 6.5. Stability diagram for a linear optical cavity. Figure obtained from Ref. [21].

The cavity mode structure is obtained by considering the behavior of the electromagnetic field contained inside the cavity, which satisfies the Maxwell's equations along with the boundary conditions imposed due to the cavity. For a linear optical cavity 
made by two spherical mirrors with radii $r_{1}$ and $r_{2}$, the resonant modes are a series of standing waves whose frequencies are ${ }^{33}$

$$
v_{q, m n}=\frac{c}{2 L}\left(q+\frac{(n+m+1)}{\pi} \cos ^{-1}\left( \pm \sqrt{g_{1} g_{2}}\right)\right) .
$$

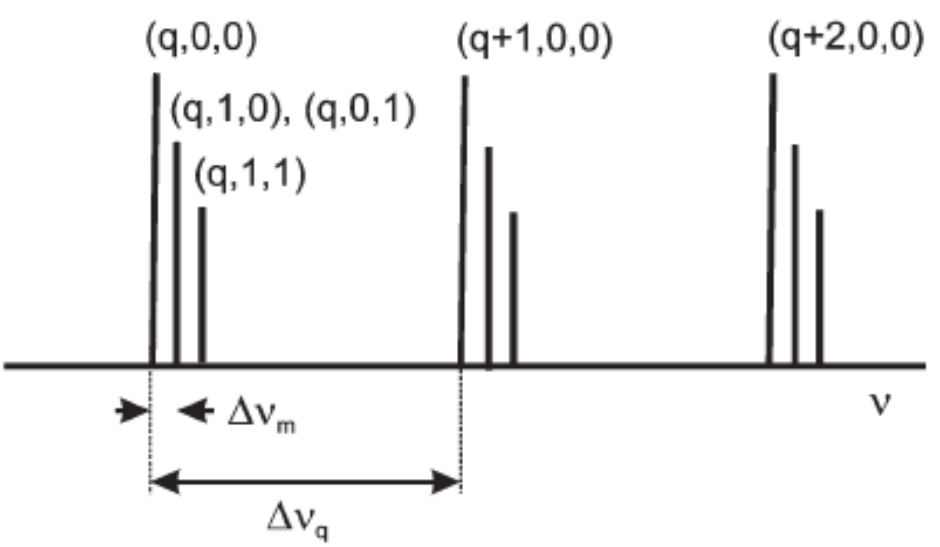

Figure 6.6. Longitudinal and transverse mode spacing in a cavity. Figure from Ref. [24].

For each set of values of the indices $q, m$ and $n$ (which are three integers), welldefined cavity modes exist. These mode indices describe the longitudinal $(q)$ and transverse ( $m$ and $n$ ) characteristics of the resonant electromagnetic fields in the cavity. Longitudinal modes determine the frequencies of radiation that satisfy the wavelength requirements of the optical cavity: that the electric vector of the light wave must vanish at the reflective surfaces of the mirrors. For a longitudinal mode to be resonant, the phase delay of a wave corresponding to a round trip within the cavity should be equal to $2 \pi q$ (where $q$ is called the longitudinal mode index). The frequency difference between two successive longitudinal modes (with the same values of $m$ and $n$ ) is the FSR. Modes with different values of $m$ and $n$ for the same longitudinal mode are called transverse modes. They quantify the cross sectional profile of the beam, i.e. the variation of the electric field in the plane perpendicular to the cavity axis and thus determine the spatial geometry of the mode. For such a mode to develop, the transverse profile must remain the same after 
one complete round trip in the cavity. These modes are referred to as $T E M_{m n}$ (Transverse Electro Magnetic) modes and are an important point of consideration for optical cavities. The lowest order transverse mode is the $T E M_{00}$ mode, which has a Gaussian cross sectional profile. Higher order transverse modes are broken up into a sub array of beams and are also less intense. The mode spacing between the transverse modes is

$$
\Delta v_{\text {transverse }}=v_{q, m+n+1}-v_{q, m+n}=\frac{c}{2 L}\left(\frac{\cos ^{-1}\left( \pm \sqrt{g_{1} g_{2}}\right)}{\pi}\right) \text {. }
$$

Thus we see that typically for a stable cavity, the transverse modes fill in the frequencies between two longitudinal modes, as shown in Figure 6.6.

A cavity can also be defined in terms of the spot sizes of the lowest order longitudinal mode in the cavity (the Gaussian $T E M_{00}$ mode). The spot sizes describe the transverse extent of the optical electric field in the cavity and knowledge of their shapes are important for experiments, particularly at the mirror surfaces and at the location of the minimum waist, i.e, the "focal point" of the cavity (for cavities made with symmetric mirrors the focal point is at the center). The spatial profiles of the beam in the cavity are found by solving the wave propagation equation for a wave traveling parallel to the optical axis of the cavity:

$$
\nabla^{2} E(x, y, z)+k^{2} E(x, y, z)=0
$$

where $k$ is the wave number of the wave ( $k=2 \pi / \lambda$, where $\lambda$ is the wavelength of the wave), $E(x, y, z)$ is the transverse profile for the complex amplitude of the electromagnetic wave, which is given by:

$$
E(x, y, z, t)=E(x, y, z) e^{i \omega t},
$$

where $\omega$ is the angular frequency of the light wave. If the optical axis of the cavity is taken as the $z$ direction, the travelling wave is 


$$
E(x, y, z)=\psi(x, y, z) e^{-i k z}
$$

$\Psi(x, y, z)$ includes a $z$ dependence, because the beam is typically focused into the cavity (as opposed to being collimated, which would have made $\Psi$ independent of $z$ ). The exact solution Eq. 6.23 depends on the geometry of the cavity. For cylindrical cavities, the solutions involve Laguerre polynomials and the resulting waves are known as LaguerreGaussian waves. For rectangular cavities, the solutions involve Hermite polynomials and the resulting waves are known as Hermite-Gaussian waves. Typical cross sectional intensity patterns for various transverse mode indices are shown in Figure 6.7.

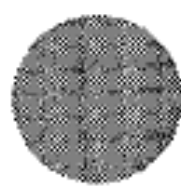

00

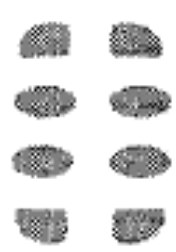

13

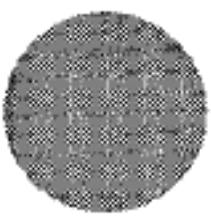

00

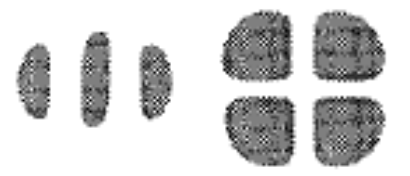

11

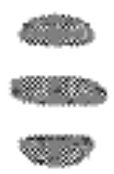

02

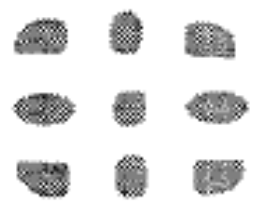

22

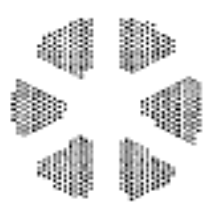

03

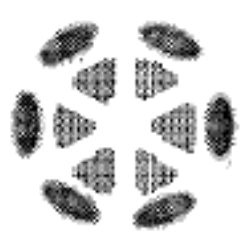

13

Figure 6.7. (a) Cross sectional intensity patterns for various Hermite-Gaussian modes. (b) Cross sectional intensity patterns for various Laguerre-Gaussian modes. Figure obtained from Ref. [21]. 
For the lowest order transverse mode, $T E M_{00}$, the beam has a Gaussian profile. At resonance, the beam forms an axial standing wave pattern such that the beam wave fronts match the radius of curvature of the mirrors at the two ends of the cavity, as shown in Figure 6.8. In the figure, $w_{1}, w_{2}$ and $w_{0}$ are the beam radii (defined as the distance from the optical axis, where the beam intensity falls to $1 / e^{2}$ of its peak value) at the two end mirrors ( $M_{1}$ and $M_{2}$, with radius of curvature $r_{1}$ and $r_{2}$ respectively) and at the waist. The beam waist is where the wave front becomes planar and for a symmetric cavity this occurs at the center of the cavity. The spot sizes at the mirrors are given by

$$
w_{1,2}=\sqrt{\frac{L \lambda}{\pi}}\left[\frac{g_{2,1}}{g_{1,2}\left(1-g_{1} g_{2}\right)}\right]^{1 / 4},
$$

and the spot size at the beam waist is given by

$$
w_{0}=\sqrt{\frac{L \lambda}{\pi}}\left[\frac{g_{1} g_{2}\left(1-g_{1} g_{2}\right)}{\left(g_{1}+g_{2}-2 g_{1} g_{2}\right)}\right]^{1 / 4} .
$$

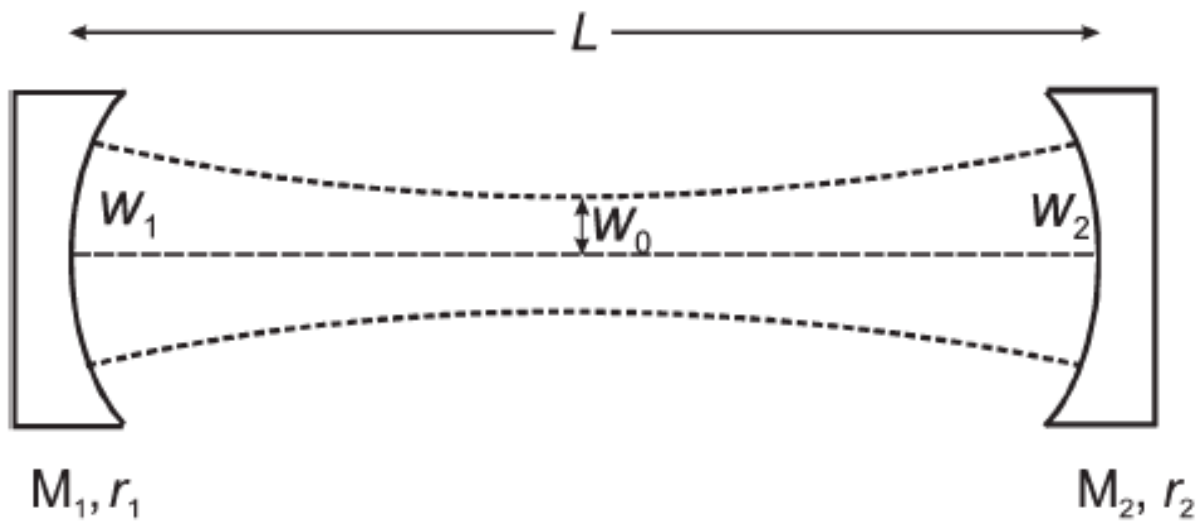

Figure 6.8. A schematic diagram of a cut through a Gaussian beam trapped in an linear optical cavity of length $L$, with two mirrors $M_{1}$ and $M_{2}$ with radius of curvature $r_{1}$ and $r_{2}$ respectively. Beam spot sizes at the waist $\left(w_{0}\right)$ and at the two mirrors $\left(w_{1}\right.$ and $\left.w_{2}\right)$ are also shown. Figure obtained from Ref. [24]. 
A near concentric cavity, with $r_{1}=r_{2} \sim L / 2$ and $g_{1}=g_{2} \sim 1$, has large spots at the mirrors, the smallest beam waist, and is very sensitive to mirror misalignments. The other case is the confocal cavity, with $r_{1}=r_{2}=L$ and $g_{1}=g_{2}=0$, which has the smallest average beam cross section along its length in the cavity and is insensitive to mirror alignment.

A convenient method of geometrically finding the position of the beam waist (or to determine if a cavity is stable) is to draw the resonator circle diagrams as shown in Figure 6.9. A typical two mirror cavity is drawn such that the mirrors with radii of curvature $R_{1}$ and $R_{2}$ are placed at a distance of $L$, the cavity length. Circles with diameter (not radii) equal to $R_{1}$ and $R_{2}$ are drawn tangential to the concave surface of the respective mirrors. If the circles intersect, the cavity is stable. The line connecting the two intersection points indicate the location of the beam waist in the cavity. If the circles do not intersect, the cavity is unstable and hence s no beam waist is defined.

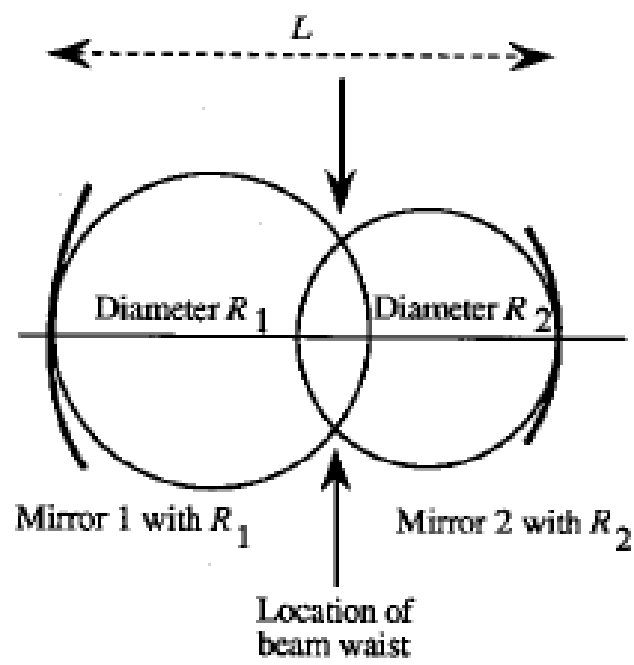

(a)

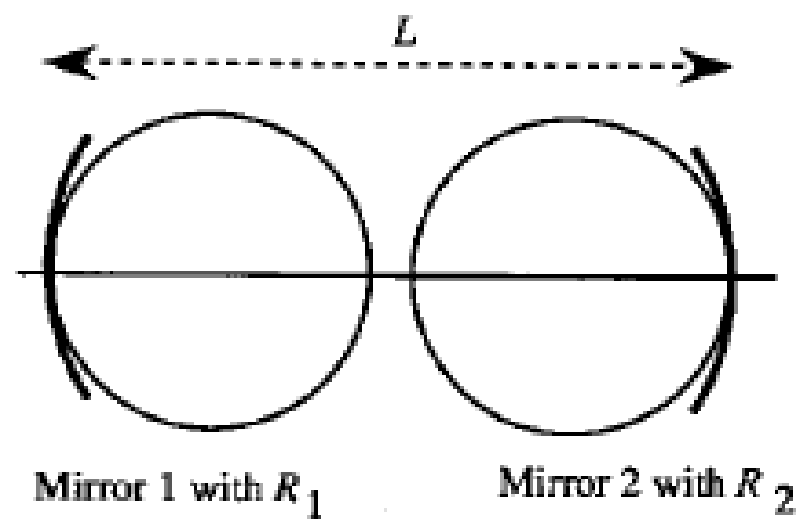

(b)

Figure 6.9. Resonator circle diagrams for two linear optical cavities, composed of two mirrors with radii of curvature $R_{1}$ and $R_{2}$ : (a) Stable cavity composed of two plano-concave mirrors. (b) Unstable cavity composed of two plano-concave mirrors. $R_{1}$ and $R_{2}$ are also the diameters of the circles drawn tangential to the mirror surfaces and $L$ is the distance between the two mirrors, defining the cavity length. Figure obtained from Ref. [21]. 
As noted previously, light will couple into an optical cavity when the laser light is in resonance with one or more of the cavity modes. Mode matching ensures the maximum coupling of the laser light into the cavity. Typically the Gaussian $T E M_{00}$ mode is preferentially excited in CRDS experiments. This can be achieved by matching the beam spot sizes in the cavity and the wave front radii of curvature of the laser radiation with the lowest order mode of the cavity. Experimentally this is achieved by placing mode matching lenses before the cavity so that the beam entering the cavity mimics the radiation field of the mode supported by the cavity. The mode matching is more effective if the laser beam has a Gaussian profile prior to injection in the cavity, since such a beam profile matches the $T E M_{00}$ mode supported by the cavity. This is referred to as "beam shaping" and is more important for CW-CRDS experiments where diode lasers are used, since diode lasers have non-Gaussian beam profiles (typically elliptical). The use of a spatial filter, which is a lens-pinhole-lens combination, in the path of the laser that is being injected into the cavity helps in beam shaping. A pair of anamorphic prisms can also make a diode laser beam more Gaussian to couple light more effectively into an optical cavity.

The preferred method of mode matching a collimated Gaussian beam is shown in Figure 6.10. A converging lens is used such that the collimated beam is focused at the center of the cavity (assuming symmetric mirrors). It can be shown using the principles of Gaussian beam propagation and paraxial ray matrices that as long as the input beam spot size is larger than the minimum beam waist diameter in the cavity (i.e. $w_{i}>w_{0}$ ), there exists a non-zero solution for " $d$ " and "f" (the distance of the lens from the end of the first cavity mirror and the focal length of the corresponding lens, respectively). Luckily for experimentalists, the solution is not a unique one, and one can choose a lens with a focal length (as long as $f>b$ ) and carefully position it to maximize the mode matching. 


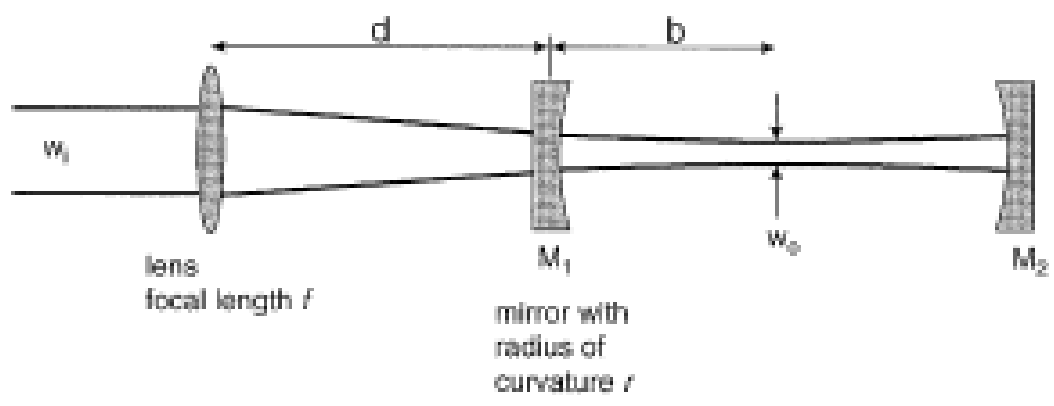

Figure 6.10. Commonly used mode matching scheme. Figure obtained from Ref. [27].

Another important issue to consider while designing an optical cavity for CRDS experiments is the effect of diffraction losses. Such losses can be approximated using the theoretical approach of Fox and $\mathrm{Li}^{34}$ The diffraction losses due to a dominant mode in an optical cavity formed by two circular mirrors, at a given wavelength, $\lambda$, is approximately:

$$
\delta_{d}=0.207\left(\frac{\lambda L}{a^{2}}\right)^{1.4} .
$$

where $a$ is the mirror radius. The effect on the ring down time of losses due to diffraction in a stable linear cavity is demonstrated in Figure 6.12, which shows the ring down times as a function of length for cavities with mirrors of various reflectivities with and without the effect of diffraction losses. The values shown in Figure 6.11 were calculated with the formulae for the ring down time in a stable linear optical cavity:

$$
\tau=\frac{L}{c\left[(1-R)+\delta_{d}\right]} .
$$

When $\delta_{d}$ is set to zero, the ring down times of a cavity with mirror losses due to only to reflectivity without any diffraction losses are obtained. The mirrors were assumed to have a radius of 1 inch and the calculations were performed for light at a wavelength of 410 $\mathrm{nm}$. The dashed lines show the effect of only reflectivity and the solid lines are the ring down times including the effect of diffraction losses. 


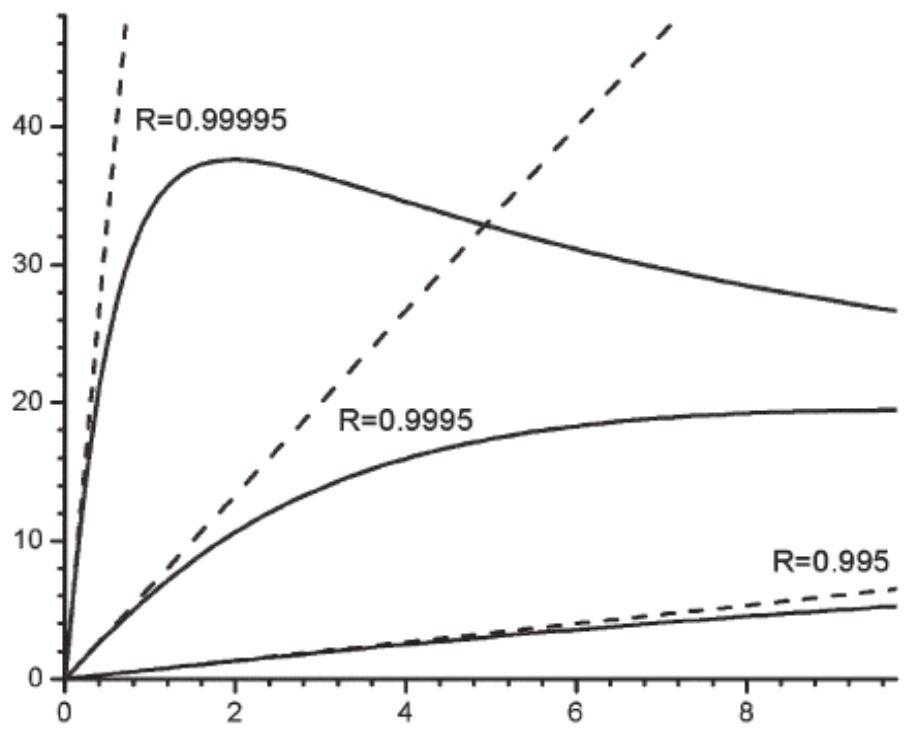

Length of the cavity (m)

Figure 6.11. The theoretical values of the ring down times of linear stable optical cavities for different mirror reflectivities, plotted against the cavity length. The dashed lines show effect of only the cavity transmissions while the solid lines take into account the effect of diffraction losses at the surface of the mirrors. Figure obtained from Ref. [24].

Even though the ring down times in a linearly stable ideal optical cavity are predicted to scale linearly with the length of the cavity, the effect of diffraction reduces the ring down times significantly and thus the linear relationship is limited to smaller range of cavity lengths. Note also that for relatively lower reflectivity values the diffraction effects are less significant.

\subsubsection{Experimental apparatus for CW-CRDS}

Excitation of a single cavity mode ensures the highest sensitivity for a CRDS experiment since the resultant intensity decay is a single exponential. Multiple longitudinal and transverse mode excitations (typical of pulsed laser CRDS experiments due to their larger bandwidth) result in a superposition of exponential decays. Interference effects in the light exiting the cavity result in intensity modulations superimposed on the ring down decay; adding noise to the signal and making it even more important to achieve the most efficient cavity excitation possible. The two main 
sources of experimental difficulty are matching a very narrow linewidth laser to the modes of the cavity and turning off the laser light being injected into the cavity once mode matching to the cavity is achieved. The first issue is accomplished by using the extensive mode matching and beam shaping techniques described previously in addition to adjusting the cavity resonant frequencies in real-time. The second challenge is accomplished with the use of a custom electronic circuit that cuts off the laser light after resonance is achieved. Figure 6.12 is a schematic diagram of a typical CW-CRDS experimental set up. The laser light is injected into the cavity only when the laser frequency overlaps with one of the resonant frequencies of the cavity. The light intensity in the cavity then increases sharply. Then the laser is switched off quickly and the ring down is observed with the detector.

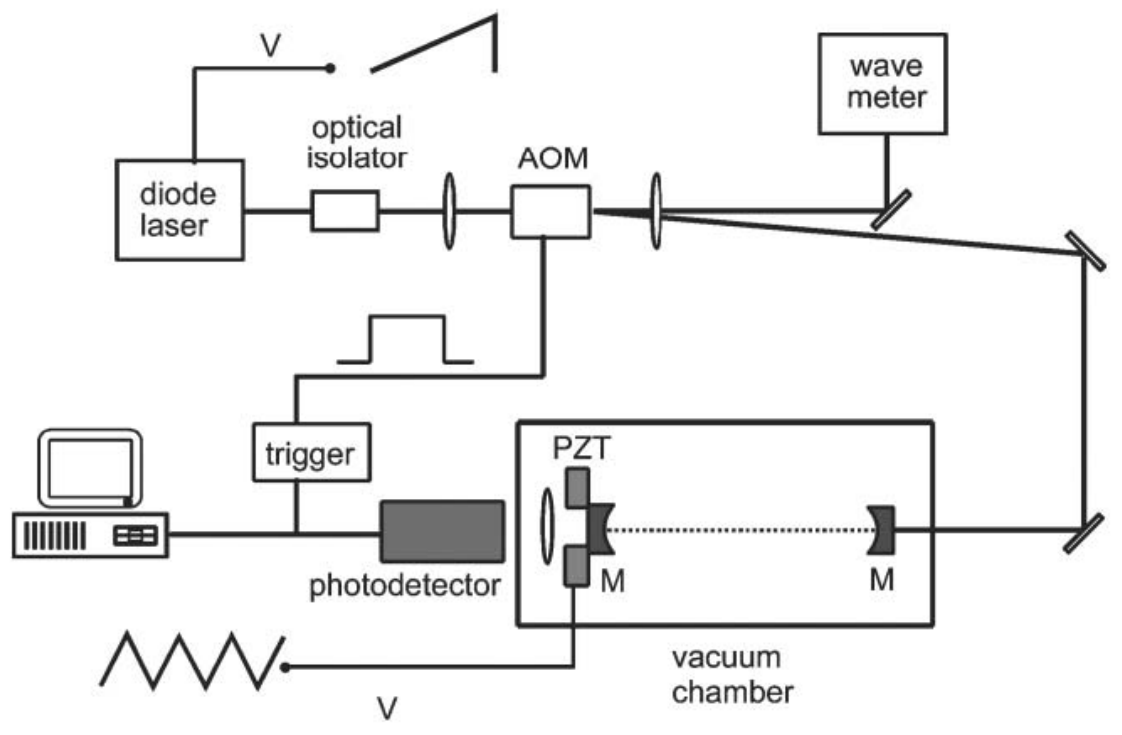

Figure 6.12. A schematic representation of a typical CW-CRDS experimental set up. Figure obtained from Ref. [24].

As noted earlier, initial measurements depended on accidental mode matching ${ }^{4,6}$ due to vibrations of the mirrors (which dither the length of the cavity). A more controlled approach is desirable. This could be achieved by modulating the frequency of the laser over more than one free spectral range ${ }^{35}$ An alternate strategy is to modulate the length of the cavity by more than half the wavelength of the laser using a PZT (piezo electric 
transducer). ${ }^{20}$ Since in these experiments, the laser beam must be rapidly (on time scales much smaller than the ring down time) extinguished once the intracavity laser light intensity is larger than a predetermined threshold level, some fast mechanism can be used to turn the laser injection "off." This can be achieved by using a Pockel's cell or an AOM (Acousto-optic modulator) as a fast switch in the path of the laser beam. Another strategy to limit the laser injection into the cavity is to sweep the mirror on the PZT fast enough so that it takes the laser out of mode with the cavity. No further injection is then possible and the intracavity light intensity decays to give the ring down signal. ${ }^{36}$ This method is known as "rapidly swept CRDS".

In Figure 6.12, we show the most commonly used technique in CW-CRDS: length modulation of the cavity to create resonances and an AOM to switch the laser off. The laser is susceptible to reflected light so an optical isolator is necessary. In the AOM a pressure transducer produces an acoustic wave that modulates the refractive index of a non-linear crystal to produce a Bragg diffraction grating pattern in the transmission of light through the crystal. The $m=1$ mode (first order diffracted beam) of the AOM is steered into the stable optical cavity after using proper mode matching optics. If the AOM frequency is high enough, the $m=0$ mode of the AOM can be used to measure the wavelength of the laser. Once sufficient light is injected into the cavity, the threshold detector triggers the AOM and it switches off, thereby extinguishing the $m=1$ mode. With the AOM off, the only mode active is the $m=0$ mode which is blocked from entering the cavity. Thus the light is deflected away from the cavity when the electronic circuit triggers and the ring down event is recorded and subsequently analyzed. The advantage of this method the diode laser remains unperturbed in this experimental scheme.

Typically the threshold detector is set such that the only the most intense, lowest order modes are detected. This ensures the expected single mode excitation of the cavity. In a CW-CRDS experiment the FSR is typically much larger than the bandwidth of the laser used, so only one longitudinal mode can be excited at a time. But, for a given longitudinal mode, as least theoretically, infinite transverse modes can be excited. The presence of higher order transverse modes can be very detrimental since they interfere and they introduce errors in measuring the corresponding ring down decay. But since the 
higher order transverse modes are low in intensity, they can be avoided by cleverly choosing a relatively high value of the threshold in the triggering circuit. In this way, even though there is the possibility of exciting higher order modes (due to cavity misalignments), ring down decays corresponding to those low intensity modes are not recorded. Observation of the transmission fringes as the cavity length is modulated or imaging the intensity using a CCD camera are the common ways to detect longitudinal mode matching. Only the ring down decays due to the highly intense $\mathrm{TEM}_{00}$ (the lowest order mode possible in the cavity) are recorded. A non-linear fit is then used to extract the time decay. In practice, to obtain an accurate value of the time constant, considerable averaging is necessary.

\subsection{Proof-of-Principle Table Top Experiments}

Before implementing CW-CRDS for an ivdf measurement in the helicon plasma source, a series of proof-of-principle measurements on a table-top apparatus in air were performed. Here we present the details of the experimental hardware and initial results from the table-top apparatus.

\subsubsection{CRDS Apparatus}

Fig. 6.13 is a schematic of the experimental apparatus used for testing CW-CRDS in air. The diode laser was tuned to $668.614 \mathrm{~nm}$, which corresponds to the Ar II transition from the $3 \mathrm{~d}^{4} \mathrm{~F}_{7 / 2}$ level to the $4 \mathrm{p}^{4} \mathrm{D}_{5 / 2}$ level. To prevent reflections from the optical components, a $60 \mathrm{~dB}$ optical isolator made by Toptica Photonics was placed between the laser and the first optical element. The light then passed through the AOM, whose chopping frequency was controlled by a function generator. The $m=1$ mode of the AOM diffraction pattern was steered into the CRDS cavity using several mirrors. An iris cut off the other modes of the diffraction pattern of the AOM. Before sending the beam into the cavity, a spatial filter was used for beam shaping.

A spatial filter is created with a lens-pinhole-lens combination. Theoretical expressions that are designed for optimum laser injection into the CRDS optical cavity assume an incident beam with a Gaussian profile. The spatial filter was used to create a Gaussian-like beam. A simple lens focused the initially non-Gaussian laser beam on to 
the pinhole. The light coming out of a pinhole forms a Newton's rings pattern due to diffraction by a circular aperture. The pinhole size was selected based on

$$
D \square \frac{2 \lambda f}{a},
$$

where $D$ is the diameter of the pinhole, $\lambda$ is the wavelength of the laser used, $f$ is the focal length of the focusing lens, and $a$ is the diameter of the laser beam. For a pinhole diameter of 50 microns, a good circular diffraction pattern with the central spot having a Gaussian beam profile was obtained. The light coming out of the pinhole was collimated with a second lens. The 50 micron diameter pinhole had to be properly positioned at the focal length of both two lenses and aligned normal to the laser beam.

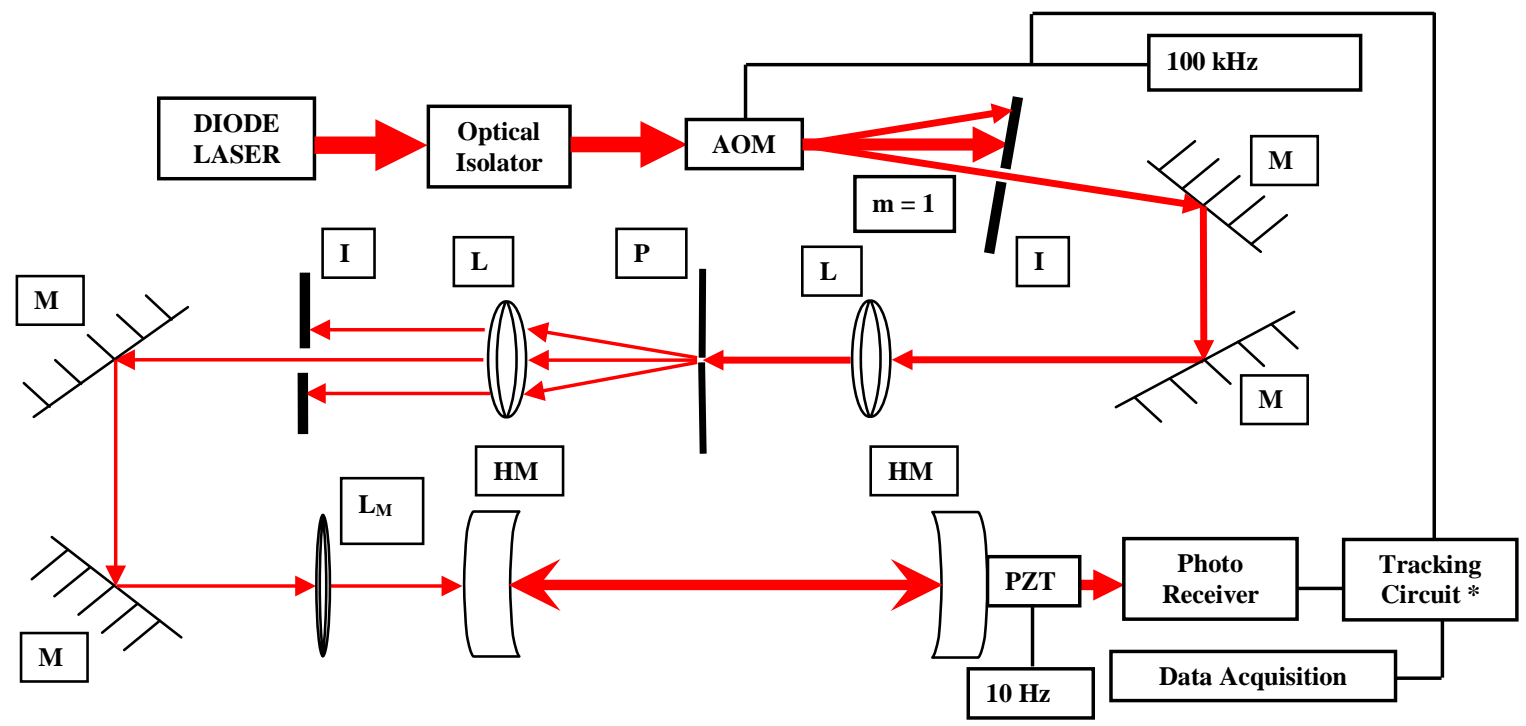

Figure 6.13. Schematic of the CW-CRDS set up used for the table-top experiment. I => iris, $M$ $=>$ beam steering mirror, $\mathrm{L}=>$ lenses used for spatial filtering, $\mathrm{PH}=>$ pinhole, $\mathrm{L}_{\mathrm{M}}=>$ mode matching lens, $\mathrm{HM}=>$ highly reflecting mirrors forming the optical cavity, AOM $=>$ acoustooptic modulator [@100 kHz], PZT => Piezo electric transducer [@10 Hz].* More details of the Tracking Circuit are given in Section 6.3.2.

The collimated light was sent through another iris so that only the central Gaussian beam of the diffraction pattern was selected. The correctly shaped beam was 
then injected into the optical cavity for CW-CRDS. Since mode matching is always critical for CW-CRDS, a mode matching lens was used to focus the light at the center of the linear optical cavity. Since the mirrors are symmetric (same radius of curvature), the minimum beam waist of the cavity was at the center of the cavity. For mode matching, the principles outlined in section 6.2.1 (see Fig. 6.10) were employed.

One of the cavity mirrors was placed on a PZT stage, Physik Instruments (PI) and controlled by a PZT-Controller made by Thorlabs. A standard function generator was used to send a triangular wave of $10 \mathrm{~Hz}$ to the PZT-Controller to modulate the cavity length by a few microns. The light transmitted through the cavity was monitored with a New-Focus photo-receiver (Model-2051) connected to an oscilloscope. The photoreceiver is a silicon diode photo detector coupled with a transimpedance amplifier. It has adjustable bandwidth up to $10 \mathrm{MHz}$ with adjustable gain. The oscilloscope has 16 bits of voltage resolution and a data sampling rate of 1 Gigasamples/s, which was sufficient in both vertical (intensity) and horizontal (time) resolution.

Monitoring the cavity transmission fringes on an oscilloscope, it was possible to observe mode matching (both longitudinal and transverse) and how changing the frequency or the amplitude of the modulation to the PZT changed the number of transmission peaks. By reducing the amplitude of the modulation (less than a micron), it was possible to select a single longitudinal mode during each PZT scan of the cavity length.

Figure 6.14 is a photograph of the table top apparatus in air. A typical transmission curve (pink curve) plotted together with the triangular voltage ramp applied to the PZT to modulate the cavity length (the yellow curve) is shown on the oscilloscope in Fig. 6.14. The modulation voltage amplitude was chosen so that only one longitudinal mode was excited during the length modulation of the cavity. The mode matching was well controlled and always occurred at the same position of both the upward and the downward ramp. As the length of the cavity was varied, the width (in time) of the resonant modes was approximately 20 microseconds. Once single mode excitation was demonstrated, the electronic circuit (see details in section 6.3.2) was introduce to trigger off the high intensity peaks and turn off AOM in a time scale faster than the calculated ring down time. 


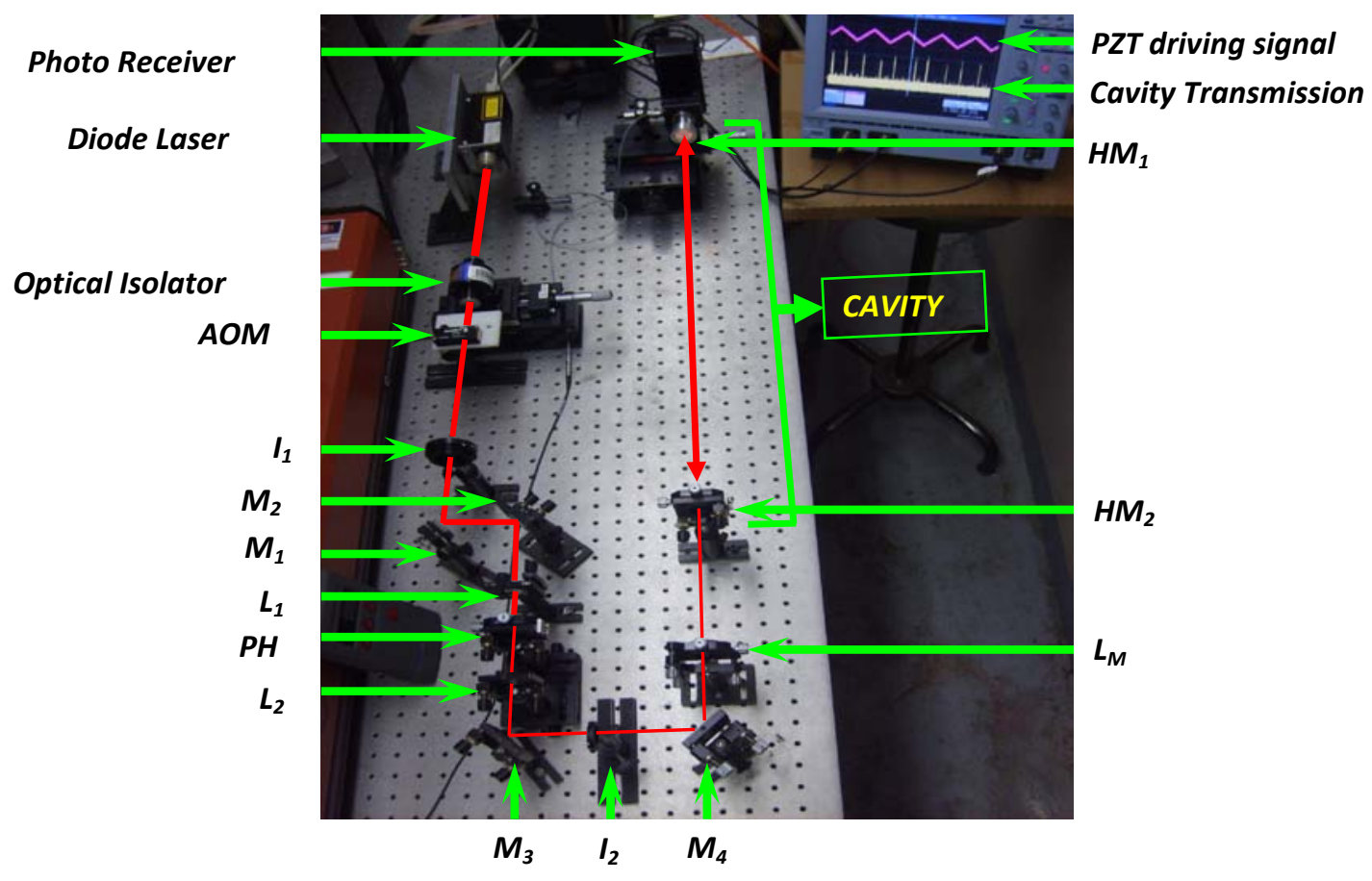

Figure 6.14. Photograph of the table top CW-CRDS apparatus to measure ring down times in air. $I_{1}, I_{2}=>$ irises; $M_{1}, M_{2}, M_{3}, M_{4}=>$ beam steering mirrors; $L_{1}, L_{2}=>$ lenses used for spatial filtering; $\mathrm{PH}=>$ pinhole; $\mathrm{L}_{\mathrm{M}}=>$ mode matching lens; $\mathrm{HM}_{1}, \mathrm{HM}_{2}=>$ highly reflecting mirrors forming the optical cavity; AOM => acousto-optic modulator (square waveform @ $100 \mathrm{kHz}$ ); PZT => Piezo electric transducer (triangular waveform @ $10 \mathrm{~Hz}$ )

\subsubsection{The CW-CRDS Tracking Circuit}

In CW-CRDS, the laser beam injected into the optical cavity must be rapidly (on time scales much smaller than the ring down time) extinguished once the intracavity laser light intensity is larger than a predetermined threshold level. The rapid shut down is achieved by using an AOM (acousto-optic modulator) as a fast switch in the path of the laser beam. The electronic, "tracking," circuit that automates the trigger-shutdown process is shown in Figure 6.15. 


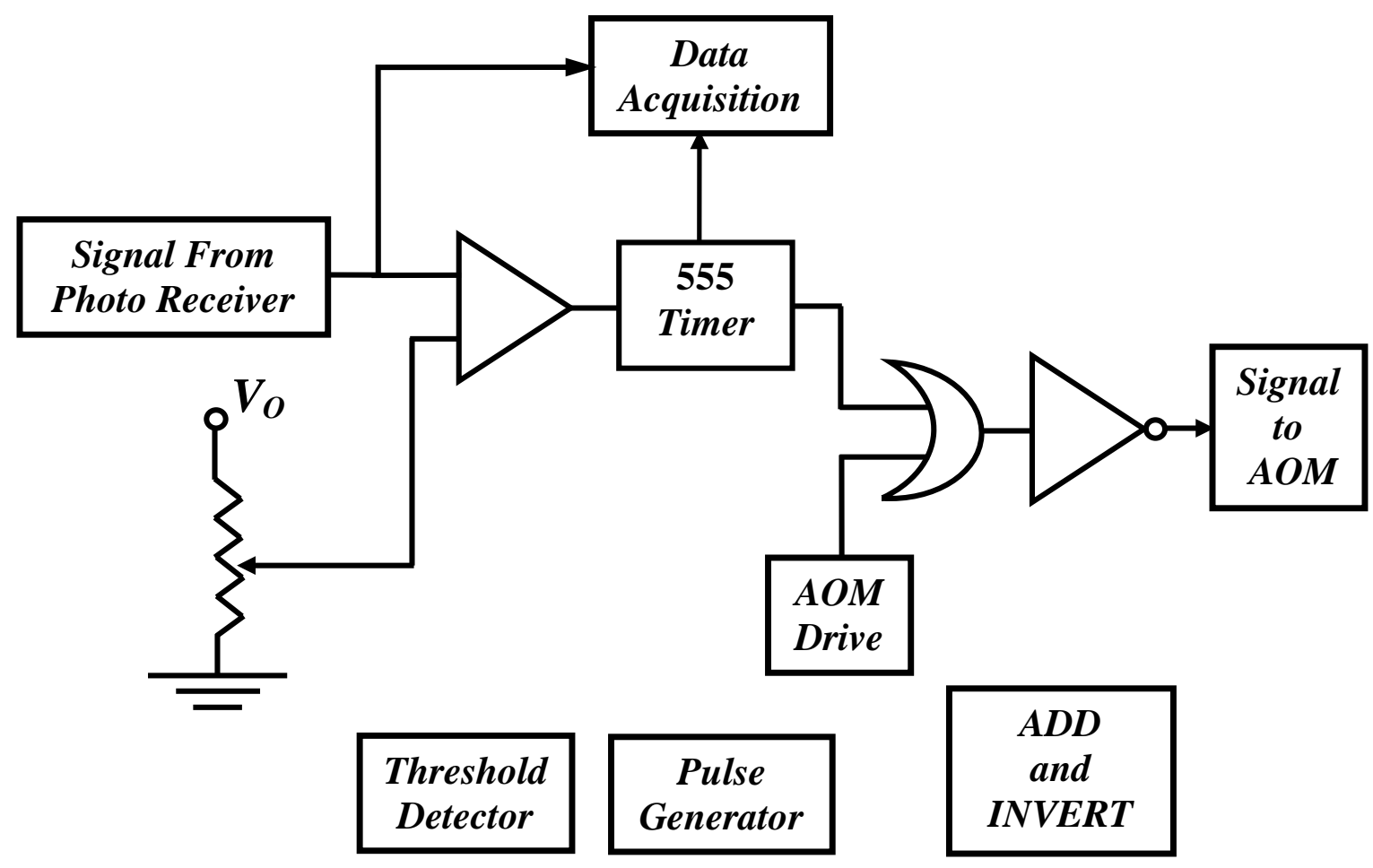

Figure 6.15. Schematic of the "tracking circuit”.

The tracking circuit consists of four electronic components (ICs) and was powered by a $0-5 \mathrm{~V}$ DC power supply. The first component of was the "threshold detector”. As a standard 9-Volt battery in conjunction with a potential finding circuit set the "threshold voltage $\left(V_{T h-}\right)$ " The voltage signal from the photoreceiver (which is proportional to the transmitted light intensity from the optical cavity) was digitized and recorded on an oscilloscope. Part of the input signal $\left(V_{i n}\right)$ was fed into the tracking circuit to be compared to the fixed (but could be externally varied by a potentiometer to be fixed at any chosen value) threshold voltage. A standard fast response comparator was designed to compare the two input signals $\left(V_{\text {in }}\right.$ and $\left.V_{T h}\right)$ and gave a positive output whenever $V_{i n}<V_{T h}$ and a negative output when $V_{i n}>V_{T h}$. This configuration yielded a negative going pulse as long as the signal from the photoreceiver was higher than the threshold detector (this state occurs during a resonance in the optical cavity). The negative going pulse triggered the second element in the tracking circuit: the 555 pulse generator. The pulse generator generates a +5 Volts TTL pulse with adjustable time. The pulse width (length of time when the voltage was high) was determined by the resistors 
and capacitors used in the 555 timer circuit. The time was typically set to be about ten times the expected ring down decay time. This 555 pulse width determined how long the laser light would be turned off, during which the ring down decay was measured.

The AOM, to function properly, needed a TTL pulse at the desired chopping frequency; provided by an additional function generator. Instead of sending the signal from the function generator directly to drive the AOM, it was added (in the time domain) to the 555 pulse. Standard AND gate digital logic was used to add the two TTL pulse trains together. A NOT gate inverted the combined signal before sending it back to the AOM driver. Thus, as soon an increase in the light intensity in the cavity occurred, the AOM driving signal was stopped for the desired length of time, thereby turning off the AOM and ensuring that the light in the $m=1$ mode of AOM diffraction pattern vanished. It is important to note that the $m=0$ mode of the AOM diffraction pattern still allows through a lot of light. Thus, the $m=1$ mode is the best choice for sending the laser light to the cavity (at the expense of reduced intensity). At the end of the 555 pulse, the laser light again passes through the AOM and the cycle repeats.

Before implementation on the optical cavity, the circuit components were tested with an artificial "resonance pulse" produced by a function generator. Fig. 6.16 shows the result of such a test. The pulse resonance width was chosen to be 20 microseconds (similar to the time scales typically found in the cavity transmission studies). From the test data, it appears that the circuit requires approximately 2 microseconds after the resonance to switch off the laser light; much shorter than the expected resonance time scale of approximately 20 microseconds. The intrinsic AOM response was very fast, about 100 ns; again, much faster than the expected ring down timescale. 


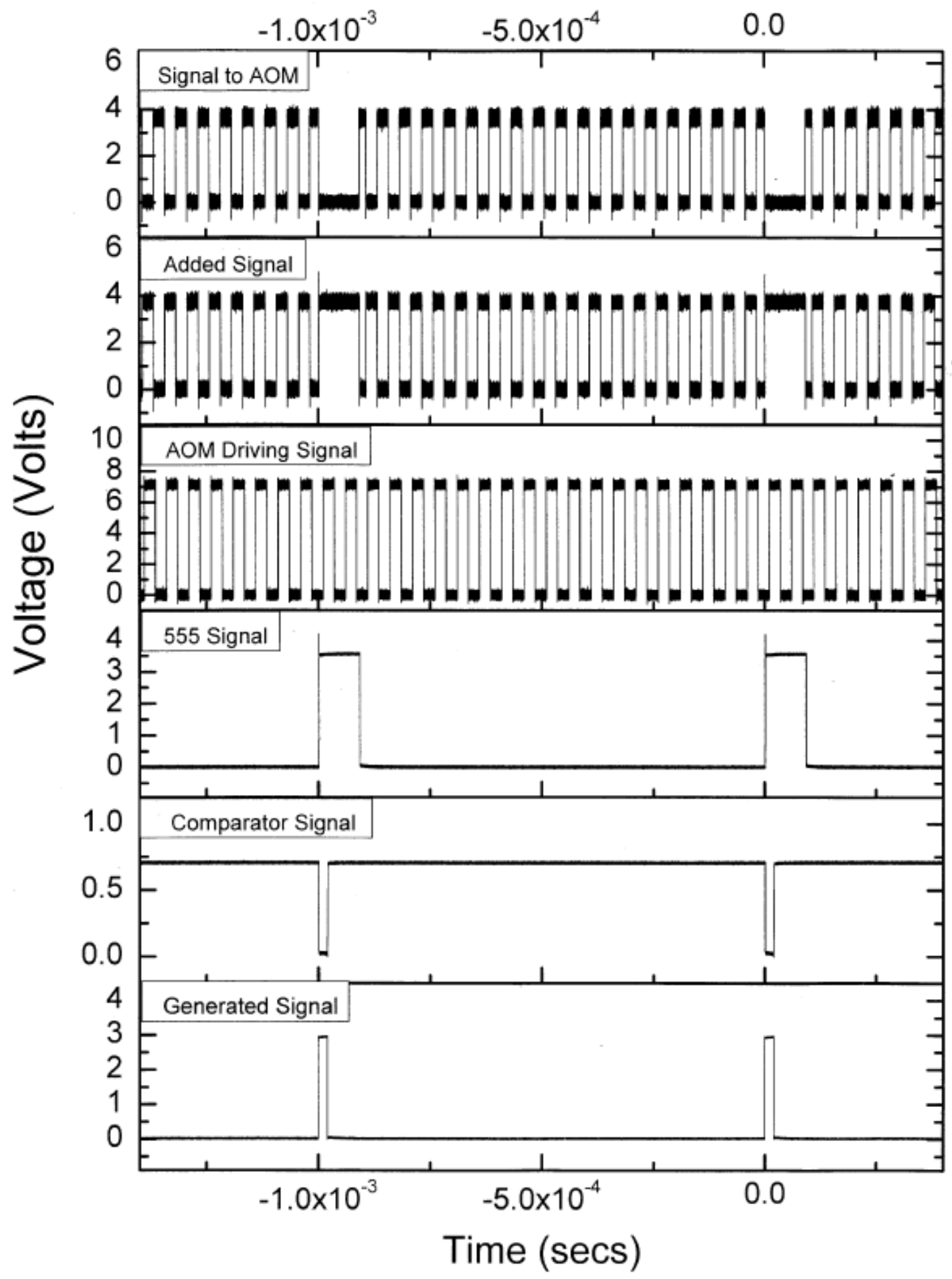

Figure 6.16. Test of the "tracking circuit" with artificially generated pulses that, resemble typical cavity resonances. The comparator triggers the 555 pulse generator as soon as the signal cross the threshold voltage (set to 2.5 Volts here).The 555 pulse width determined for how long the AOM drive signal is turned off. 


\subsubsection{Results and Discussion}

Shown in Fig. 6.17 for the complete CW-CRDS table-top experiment is the cavity transmission (black curve) and the driving signal for the AOM (blue curve) on a common time axis. The AOM shuts off as soon as the cavity output voltage exceeds the threshold voltage set on the comparator. The AOM blocks the laser light long enough for the ring down decay to be recorded. For cavity transmission peaks smaller than the threshold voltage, the tracking circuit does not.

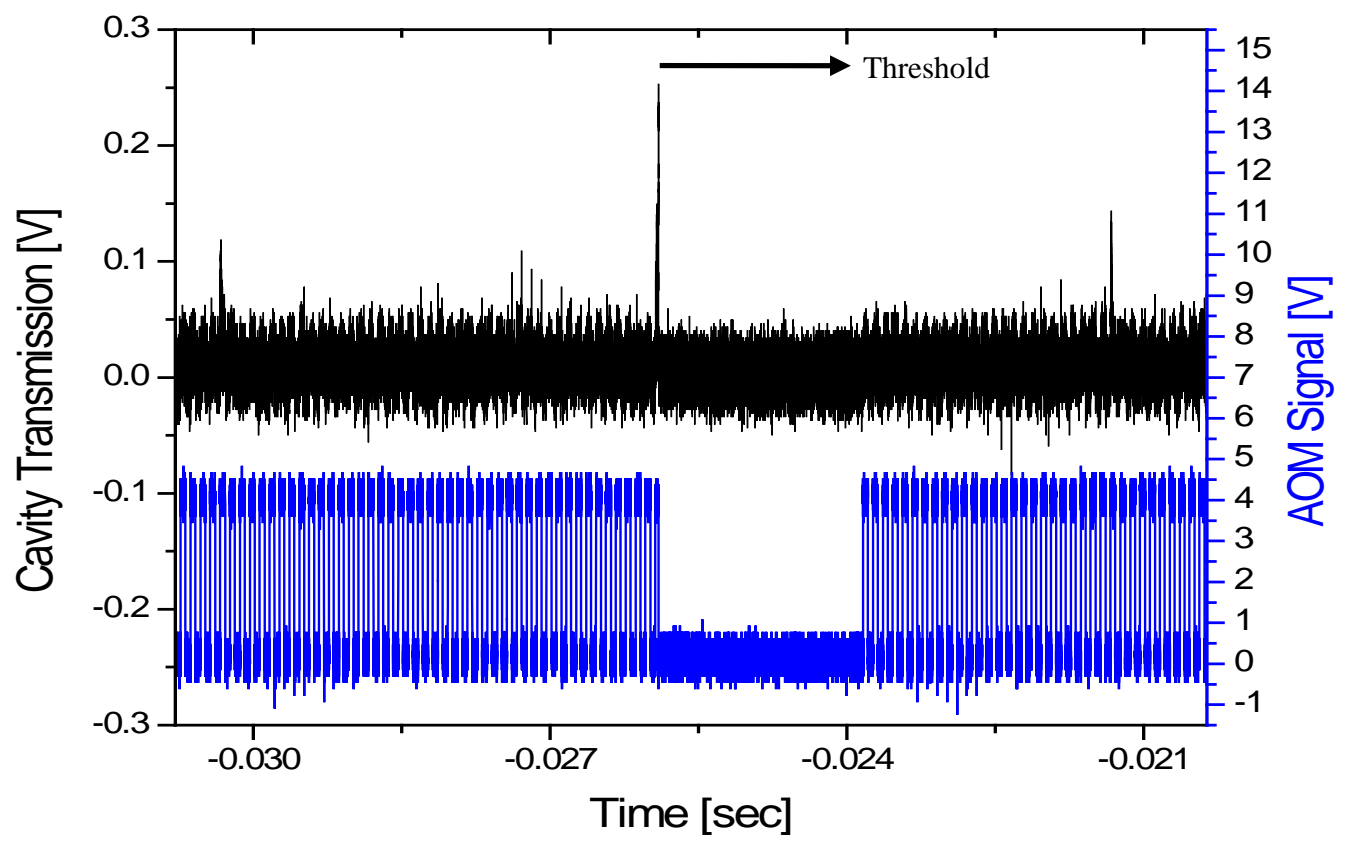

Figure 6.17. The black curve is the cavity transmission and the blue curve is the composite AOM driving signal. The AOM is turned off as soon as the cavity output exceeds the threshold voltage but remained unaffected by the smaller peaks.

Figure 6.18 is an expanded view of a portion of Fig. 6.17 that shows the speed of the response of the AOM. Note that small amplitude oscillations in the cavity transmission before the tracking circuit triggers are evident in the photoreceiver signal.. The absence of the same oscillations in the cavity transmission after the circuit triggers, confirm that the shut off AOM is blocking the entire light incident on the cavity. 


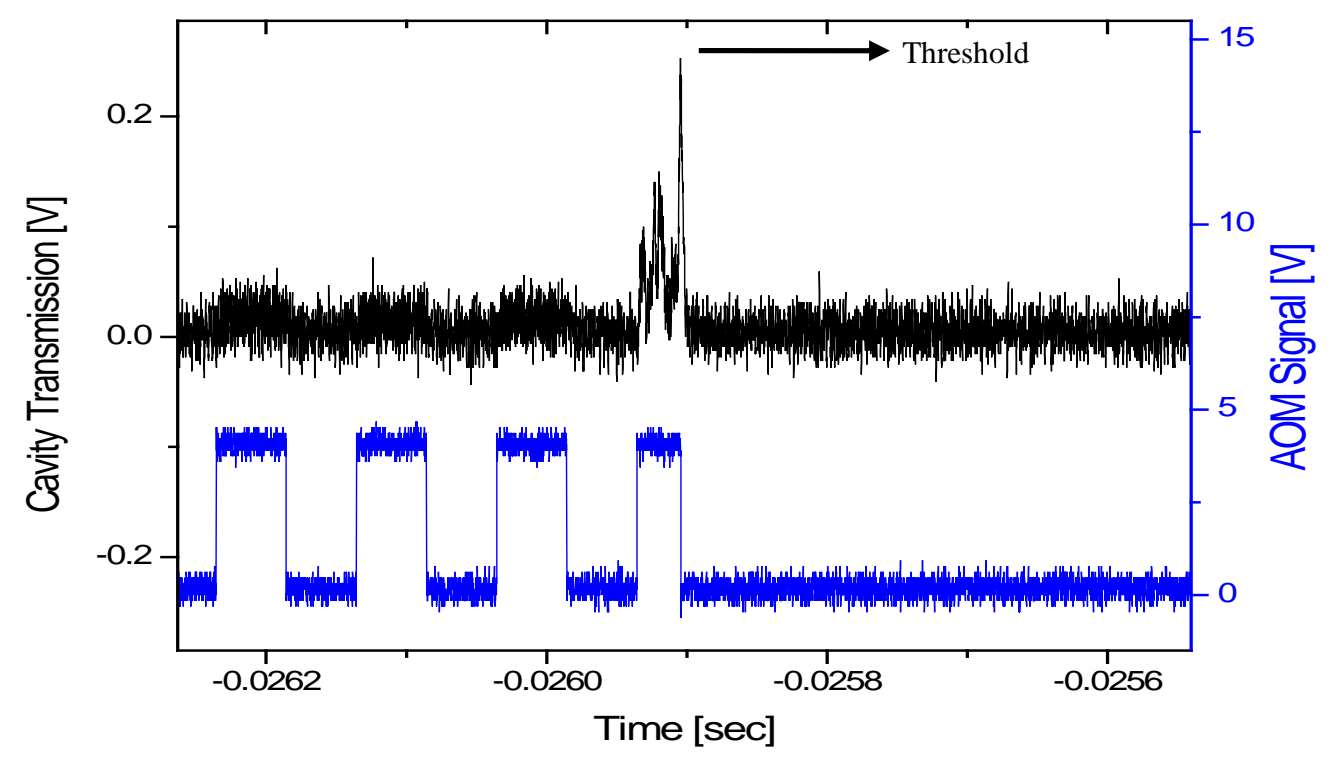

Figure 6.18. The black curve is the cavity transmission and the blue curve is the signal sent to the AOM. The AOM is turned off as soon as the cavity output voltage exceeds the threshold voltage.

\subsubsection{Table-Top Experiment Ring Down Time Measurements}

As preliminary measurements found ring down decay times for an empty cavity that were three times smaller than expected based on theoretical calculations, ring down times were determined for a range of cavity lengths. Over the range of cavity lengths examined, the ring down decay time is predicted to be linear with length. Fig. 6.19 shows ring down curves as the length of the cavity was changed from a very short cavity (10 $\mathrm{cm})$ to the longest that could be achieved with the experimental apparatus $(130 \mathrm{~cm})$. 


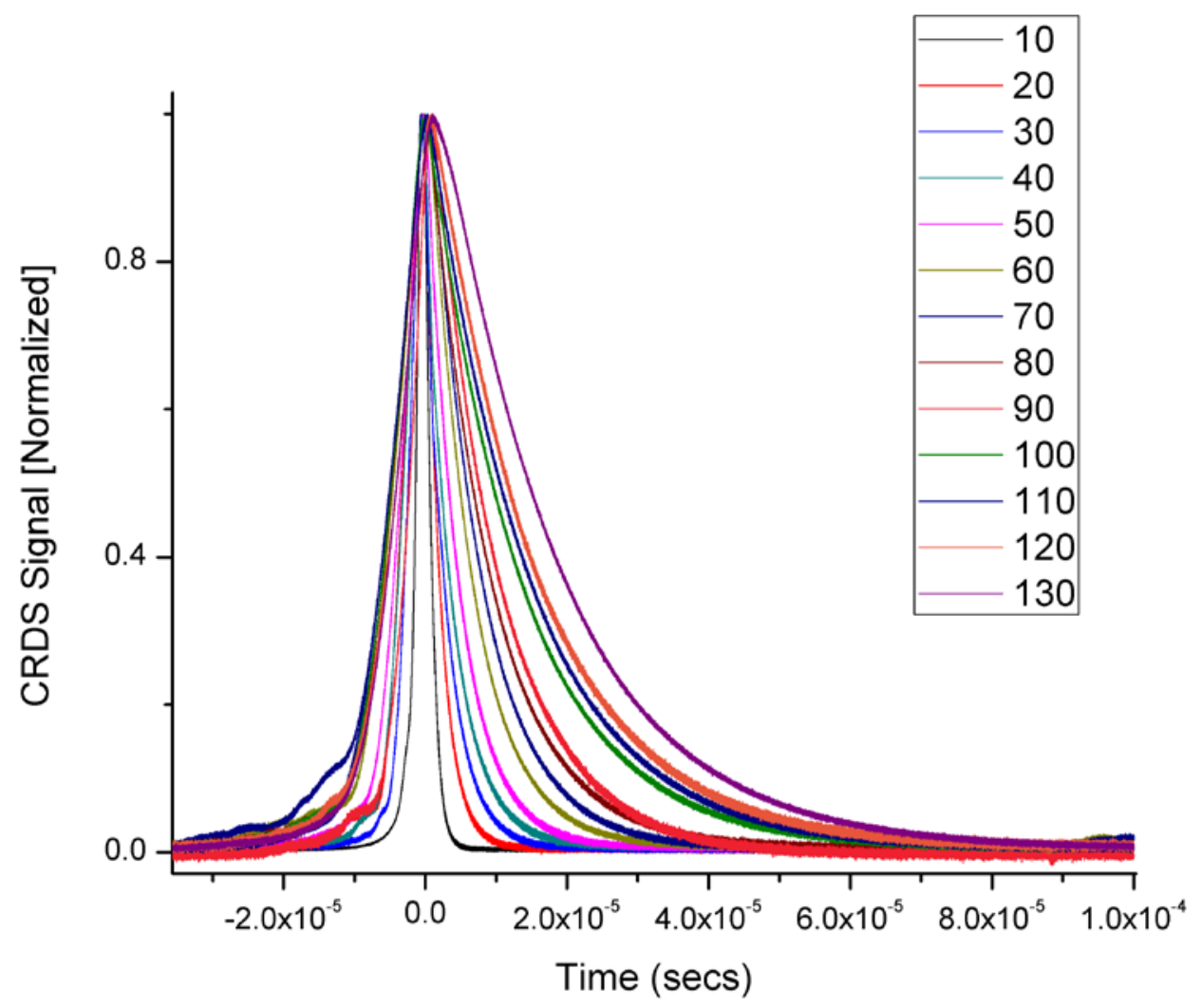

Figure 6.19. Ring down decay curves shown as a function of cavity length.

Shown in Figure 6.20 are the measured ring down times as a function of cavity length extracted from the data of Fig. 6.19 and a linear fit through the data. The linear fit gives an effective reflectivity of $R_{\text {eff }}=99.976 \%$ for the mirrors; significantly smaller than the vendor quoted value of $R=99.995 \%$. The lower reflectivity results in ring down times that are approximately three times smaller than expected. 


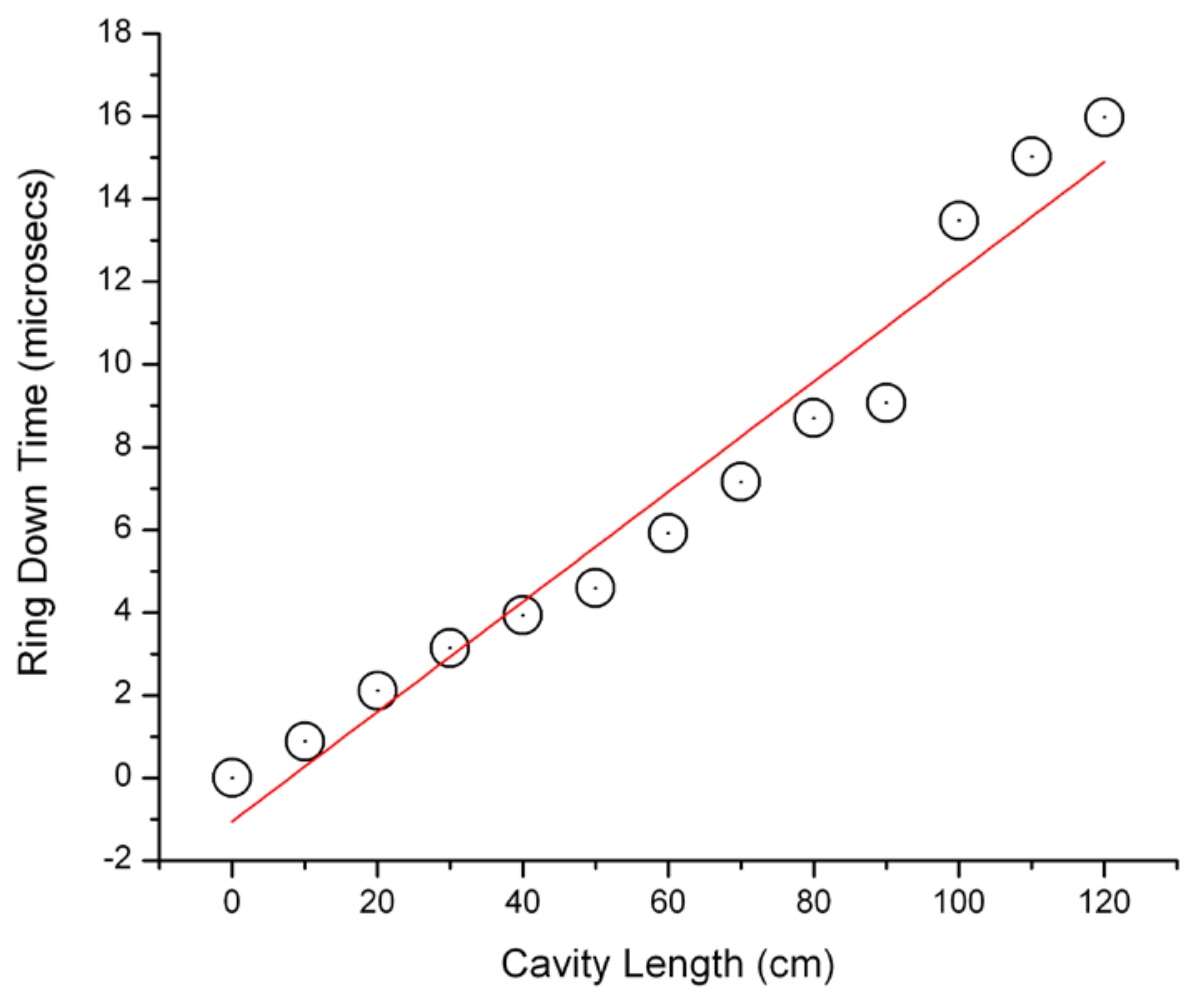

Figure 6.20. Ring down times versus the length of the cavity.

\subsection{CW-CRDS IVDF Measurements in CHEWIE}

Having successfully tested the CW-CRDS scheme on t, the CW-CRDS apparatus was installed on CHEWIE. A critical step in the CHEWIE installation was the design and construction of adjustable mirrors mounts that coupled the laser light into the cavity while simultaneously serving as vacuum windows for the plasma chamber.

\subsubsection{Mirror Mount Design}

Figure 6.21 shows schematics of the mirror mounts designed and fabricated in the WVU machine shop (Fig. 6.22 is an actual picture of the assembled mirror mounts). Only the welded adjustable bellows and the electrical feedthrough were purchased commercially. The mounts were so designed that one of the mirrors could be placed on a PZT, for cavity length modulation, while maintaining vacuum. The PZT required an external controlled signal; supplied through a vacuum SMA feedthrough. The PZT 
electrical fitting was a Lemo 00 connector, therefore a vacuum compatible Lemo 00 to SMA coupler connected the vacuum feedthrough to the PZT. The PZT controller output had a SMC fitting, so a SMA to SMC coupler was used between the PZT controller and the vacuum feedthrough. The vertical screws shown in Fig. 6.21 (and springs not shown in the figure) were used to support the weight of the bellows and to provide some adjustment of the angle of the mirrors. Fine adjustment of the mirrors to ensure single mode matching for CW-CRDS was accomplished with the extremely fine thread horizontal screws show in the figure. When not in use, the highly reflective mirrors were protected from the plasma with gate valves. The mirror mounts were designed to be mounted to any of the plasma devices in the WVU laboratories through $23 / 4$ " or 6" Conflat ${ }^{\mathrm{TM}}$ flanges. When mounted the cavity length was $85 \mathrm{~cm}$.

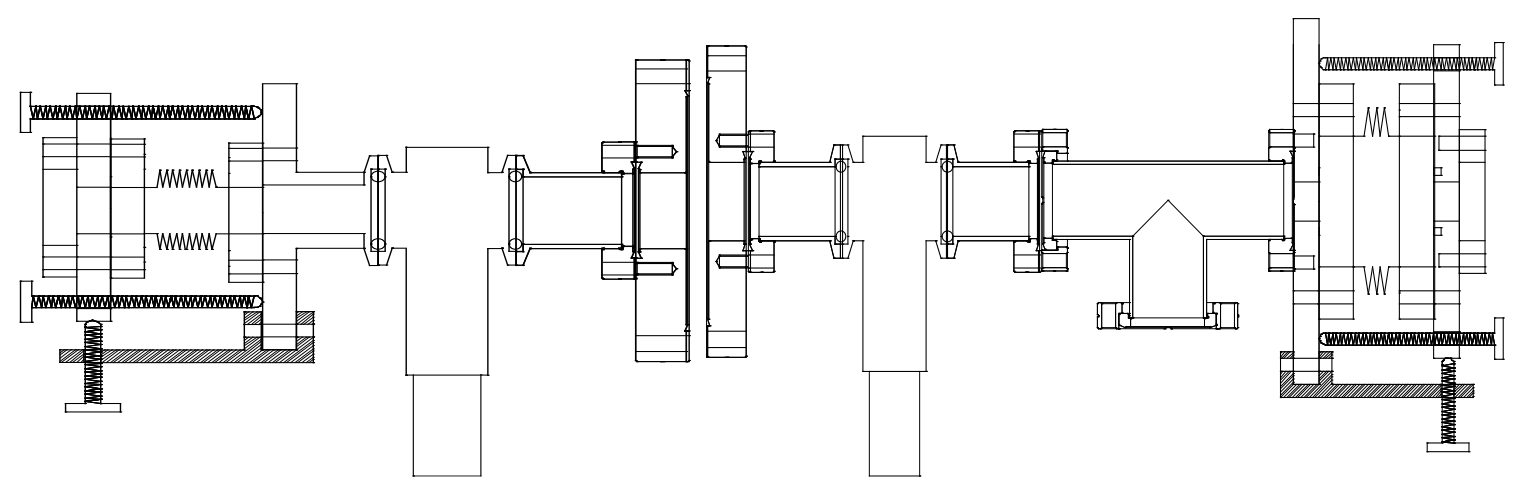

Figure 6.21. Mirror mount assembly for creating a resonant cavity on CHEWIE.
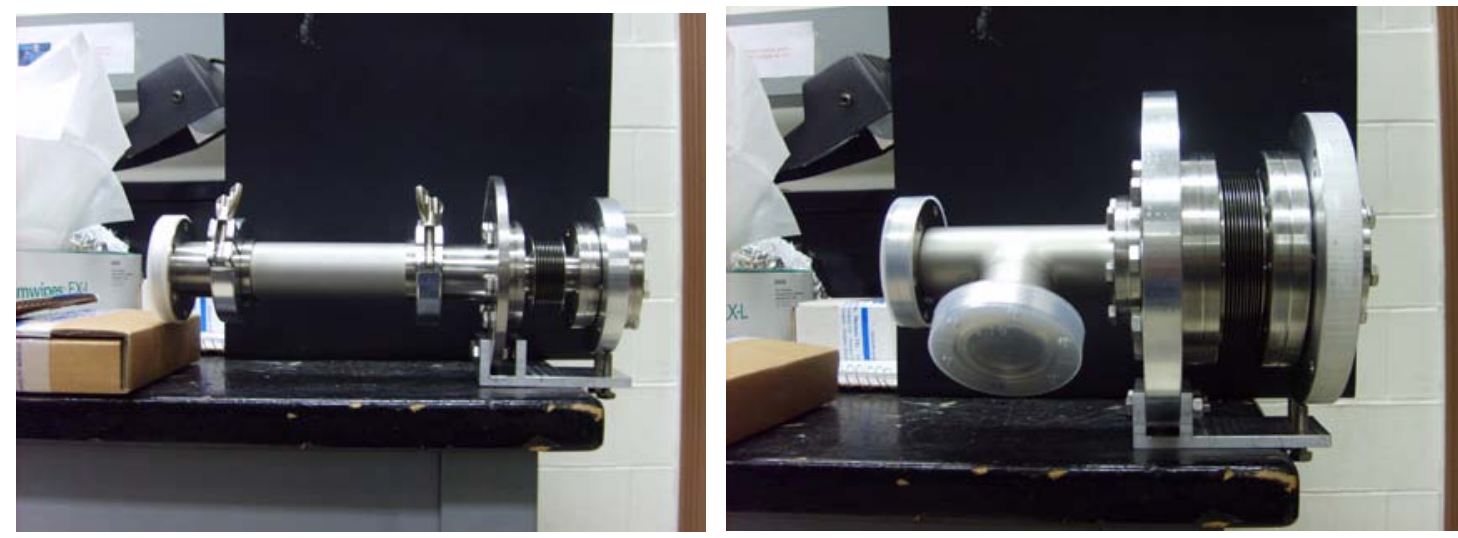

Figure 6.22. Photographs of the fully assembled mirror mounts. 


\subsubsection{Experimental Apparatus for CRDS in CHEWIE}

To accomplish CW-CRDS in CHEWIE, the laser beam is injected (after beam shaping and mode matching) into the optical cavity (made by the mirrors mounted on CHEWIE) that surrounds the plasma and the transmitted light is detected by the photoreceiver which then triggers the tracking circuit. Figure 6.23 shows a photograph of the CHEWIE system with the CRDS components installed. The laser, along with all the optical components necessary for proper laser light injection, is on the left on a pneumatically isolated optical table. The same configuration as described in Section 6.3.1 (Figure 6.13) was used for the CHEWIE experiments. The laser light passes through an optical isolator and then through the AOM. $10 \%$ of the laser is split off before the AOM and injected into an optical fiber for coupling into a wavemeter; used to record the wavelength of the laser for each scan through the absorption line of argon ions. The highly elliptical laser light is shaped into a Gaussian beam by the spatial filter and the light is then injected into the optical cavity through the mode matching lens of focal length $75 \mathrm{~cm}$. As shown in the photograph, the mirrors are mounted towards the lower part of CHEIWE (port BB' in Figure 2.9). The transmitted light passes through a $10 \mathrm{~nm}$ filter and is then collected by a lens which focuses the light on to the photoreceiver. Each ring down curve is recorded on a digital oscilloscope with 16 bits of vertical resolution. An example of a typical ring down curve and the corresponding trigger signal (the output

of the 555 pulse generator described in Section 6.3.2) are shown on the oscilloscope in the photograph. 


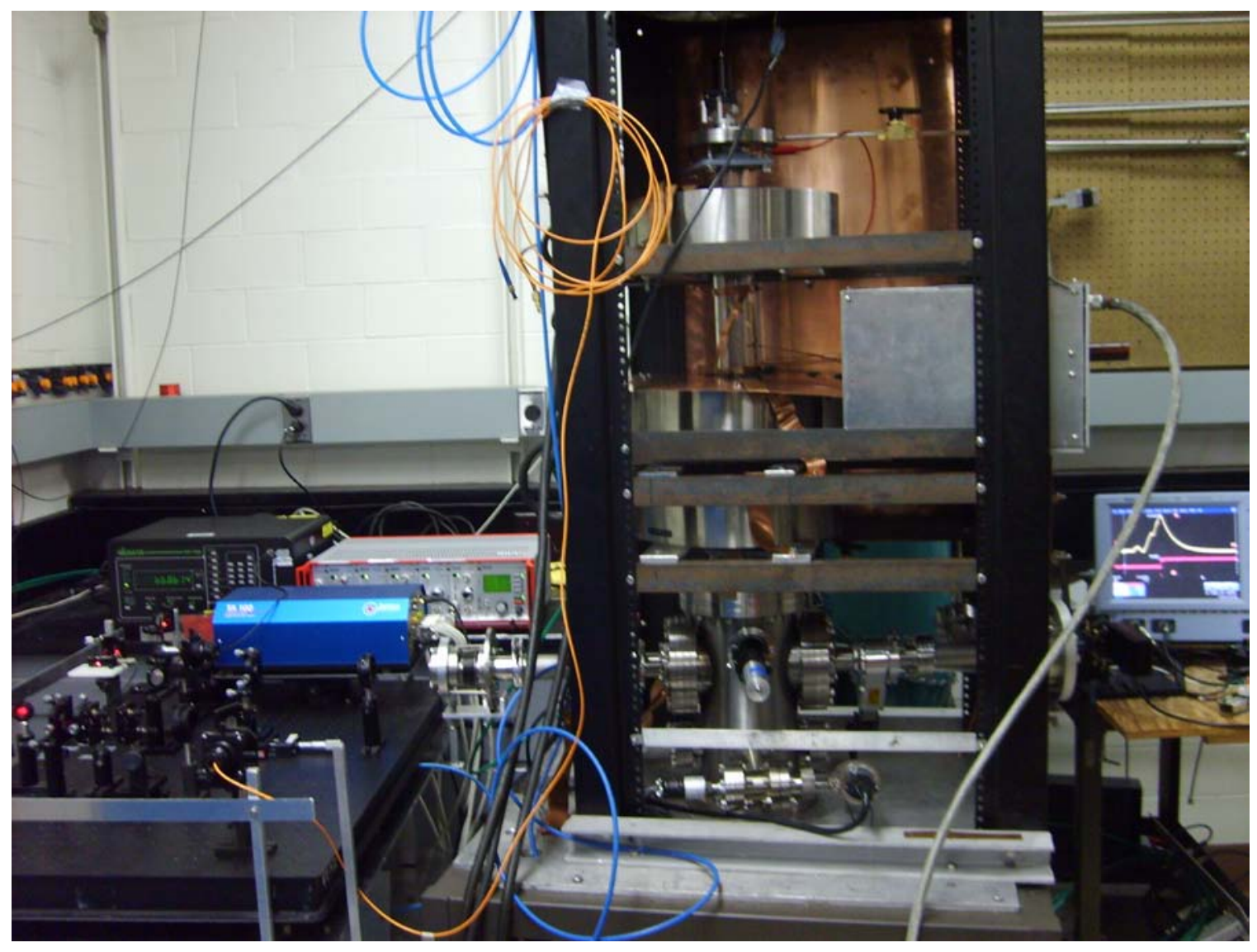

Figure 6.23. CW-CRDS apparatus for IVDF measurements CHEWIE.

\subsubsection{Results and Discussion}

To obtain a ring down measurement, the cavity had to be aligned for single mode excitation the intensity of the transmitted signal had to be large enough to trigger the tracking circuit. Figure 6.24 shows data from a step in the alignment process. The cavity transmission is shown in red and the corresponding trigger signals generated by the mode resonances are shown in green. When the CHEWIE vacuum pumps are on, the vibrations permit the excitation of a considerable number of additional transverse modes. By increasing the threshold for the tracking circuit, only the most intense, purely longitudinal modes, trigger a ring down measurement (see Fig. 6.24). The high-order transverse modes yield much smaller resonance peaks and are ignored by the electronics. 

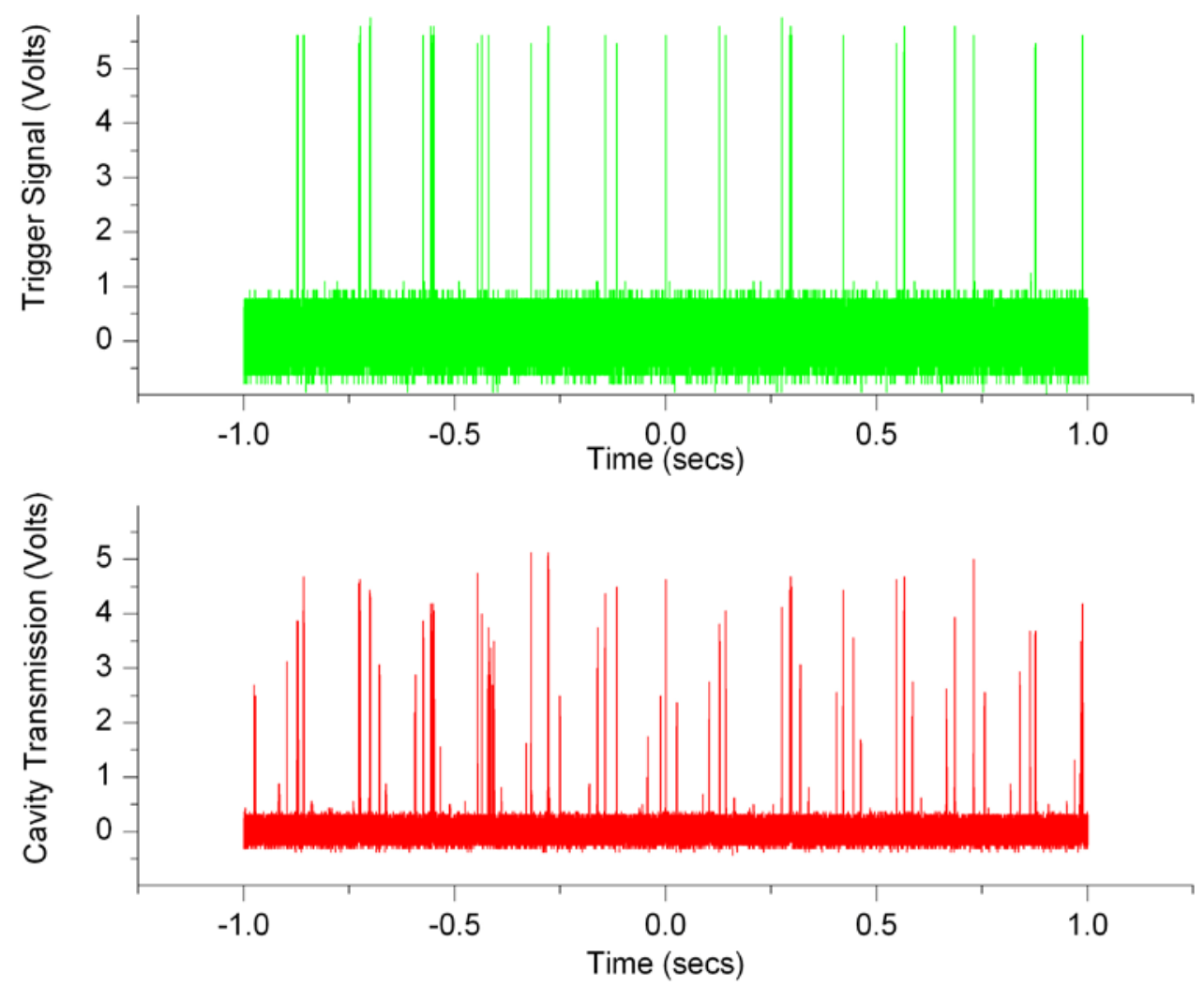

Figure 6.24. Example of transmission due to cavity mode matching (in red) and the corresponding trigger signals generated to shut off the AOM to begin a ring down measurement (in green).

Example ring down curves obtained during plasma operation in CHEWIE are shown in Figure 6.25. Note that the measurement is very reproducible. Each ring down curve was averaged over 50 individual ring down decays. The decay times determined by exponential fits to the measured curves are roughly 5 microseconds. The statistical error in the measurements, based on the standard deviation of the measurements, is of the order of 0.02 microseconds. 


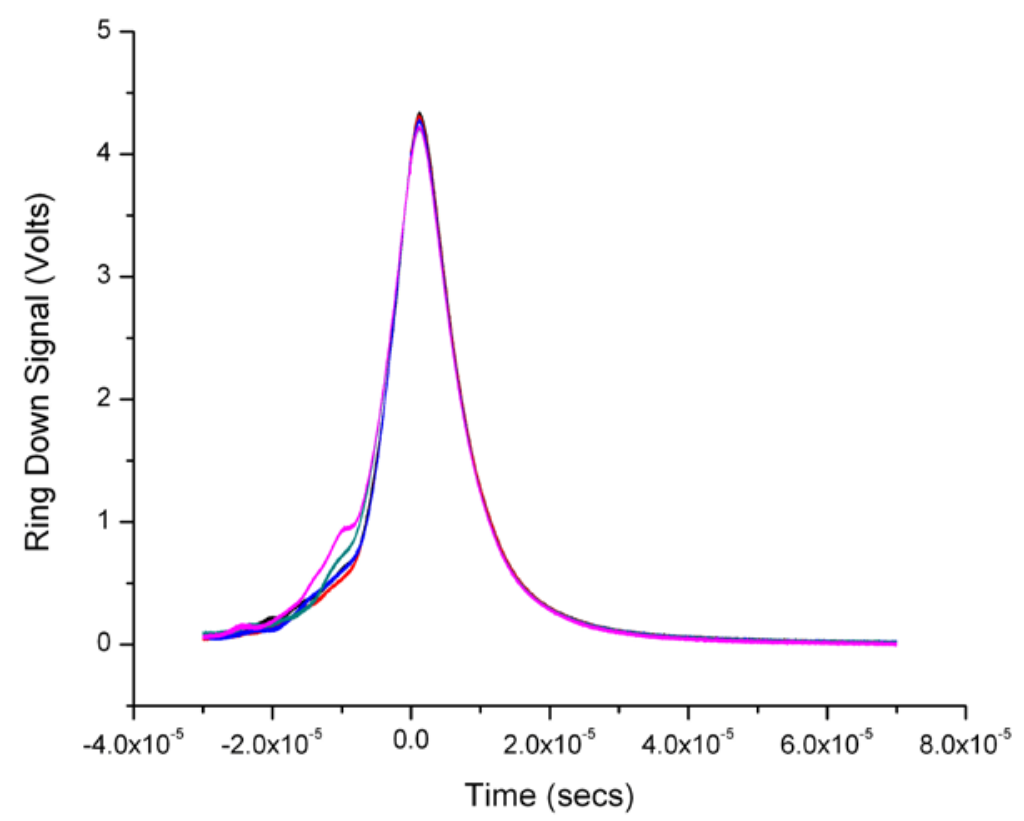

Figure 6.25. Example of the consistency of cavity ring down signals in CHEWIE obtained at different times. Each curve is an average of 50 ring down decays.

\subsubsection{CW-CRDS Measurements of Ar II IVDFs}

During CW-CRDS experiments on CHEWIE the electromagnets on CHEWIE would routinely overheat (each CW-CRDS measurement required many of tens of minutes). To reduce overheating problems, the topmost magnet in CHEWIE was removed from the electrical circuit. An extra fan was also added near the antenna to keep the antenna and the Pyrex tube cooler during the experiment. Plasma was generated in CHEWIE with a magnetic field of 1 kGauss, an antenna frequency of $10 \mathrm{MHz}, 1 \mathrm{kWatts}$ of forward power, and at an argon pressure of $3 \mathrm{mTorr}$ (at $16 \mathrm{sccm}$ ).

Each ring down measurement was averaged over one hundred ring down decays before a non-linear exponential fitting routine was used to determine the time constant of the decay. At least ten such ring down time measured were then averaged together. It was hoped that the heavy averaging would reduce the errors in each measurement. Unfortunately, the largest errors in the measurement process were not random errors.

As explained previously, CRDS is a differential measurement technique. Ring down time measurements with and without analyte are needed to measure the 
characteristics of the target absorption line. Ring down times are expected to be smaller when the plasma is turned on and the difference in the on-off plasma states is expected to increase near the absorption line. The sensitivity of the experiment is determined by the ability to statistically distinguish the ring down times between these on-off plasma states. In these experiments, very consistent ring down times with the plasma off were observed. However, when the plasma was turned on, the ring down times appeared to continuously decrease. The decreasing ring down times along with measurements without plasma are shown in Figure 6.26. The nearly monotonic decrease in the ring down times during the plasma "on" shots indicated a significant systematic error. The most likely explanation for the decrease in ring down time is that contamination from plasma affected the mirrors, leading to a reduction in their reflectivity. The ring down time is very sensitive to the reflectivity of the mirrors and a slight reduction in the mirror reflectivity causes a large reduction in the ring down time. After the plasma was turned off, the ring down time increased but never fully recovered (see Fig. 6.26). Given that windows on helicon plasma sources routinely get coated with metallic films, it is not too surprising that the CRDS mirrors are getting coated during plasma operation. So we could not simply take the difference in the ring down times between the averaged plasma on and off states.

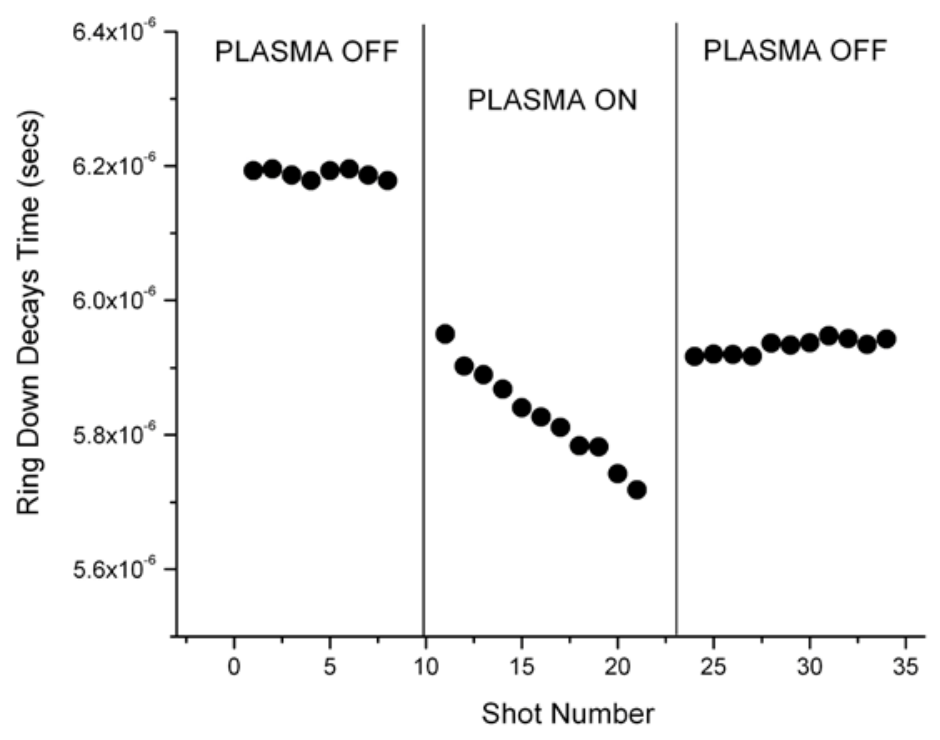

Figure 6.26. Typical behavior of ring down times with and without plasma in the optical cavity. 
In spite of the degradation of the mirrors, the difference in ring down times between the on and off plasma states is consistent between the beginning and end of each experimental run. Therefore, to obtain a measure of the difference of the plasma "on" and "off" states, the difference between a linear fit to the plasma on measurements and a two point linear fit to the plasma "off" cases before and after the plasma run was calculated for all the wavelengths across the spectral line of interest. The target ion state investigated was the argon ion transition at $668.6138 \mathrm{~nm}$ (in vacuum), which is a standard initial state for conventional LIF. Figure 6.27 shows the Ar II ion velocity distribution measurement obtained with CW-CRDS in CHEWIE.

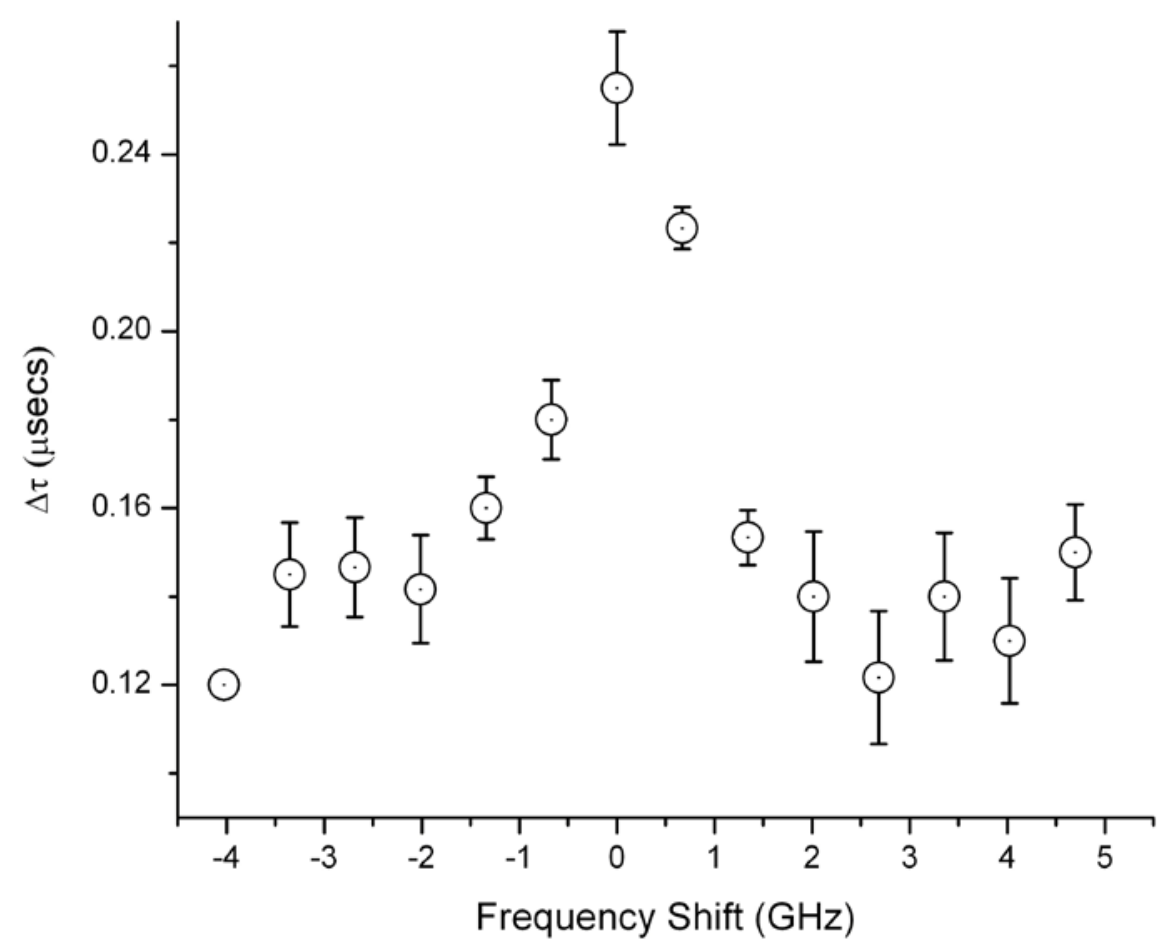

Figure 6.27. Ar II ion velocity distribution function measured with CW-CRDS in CHEWIE.

The length of the optical cavity in CHEWIE is $85 \mathrm{~cm}$ and the plasma fills $20 \mathrm{~cm}$ of the cavity (the distance between the two ports in the $15 \mathrm{~cm}$ diameter expansion 
chamber). From Eq. 6.14 and the data shown in Fig. 6.27, the absorption coefficient for argon ions in this state is $(5.1 \pm 0.2) \times 10^{-7} \mathrm{~cm}^{-1}$. The argon ion absorption cross section at $668.6138 \mathrm{~nm}$ is $8.5 \times 10^{-13} \mathrm{~cm}^{2}$. From the measured absorption coefficient and the integral of the measured IVDF (Fig. 6.27), the metastable argon ion density is determined to be $(1.2 \pm 0.03) \times 10^{6} \mathrm{~cm}^{-3}$. Note that this is the average metastable density along the laser path in the cavity.

\subsubsection{Comparison of CW-CRDS and LIF measurements of Ar II IVDF.}

As a check of the CW-CRDS measurements of the IVDF in CHEWIE, the IVDF was also measured with a standard LIF technique for the same plasma conditions. ${ }^{37}$ Shown in Figure 6.28 are the Ar II ion velocity distribution functions obtained with CWCRDS and LIF in CHEWIE.
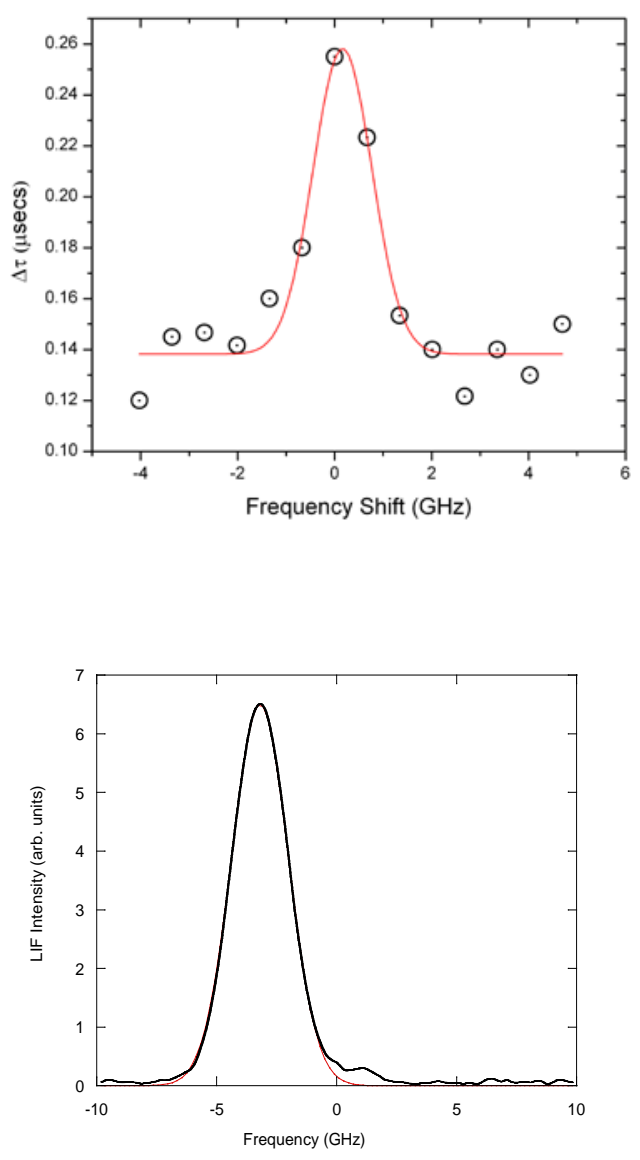

(a)

(b) 
Figure 6.28. Comparison of Ar II ion velocity distribution functions measured with CW-CRDS and with LIF in CHEWIE.

The ion temperature can be extracted from a purely Doppler broadened IVDF by fitting Eq. 6.31

$$
I(v)=I\left(v_{0}\right) e^{\left(\frac{-0.0925 *\left(v-v_{0}\right)^{2}}{T_{\text {Argon }}}\right)},
$$

to the measured absorption line shape, where $I_{0}$ is the peak intensity, $v$ is the laser frequency, $v_{0}$ is the center frequency, $T_{\text {Argon }}$ is the ion temperature, and the coefficient in the exponential, 0.0925, is for the 668.6138 argon ion transition. ${ }^{38}$ The appropriate fitting coefficient, 0.0779 (corresponding to the dye laser wavelength of $611.6616 \mathrm{~nm}$ that was used for taking the LIF measurements), was used for the LIF measured IVDF shown in Fig. 6.28b. The ion temperature measured with CW-CRDS was $0.09 \pm 0.02 \mathrm{eV}$, whereas the LIF measurements yielded a value of $0.21 \pm 0.02 \mathrm{eV}$.

Note that the LIF measurements were obtained with a lengthy integration time constant compared to the laser sweep timescale and therefore the ion temperature is significantly overestimated in the LIF measurement. ${ }^{39}$ This can account for an error up to $20 \%$ of the measuremed temperature values using LIF. Moreover, due to the unavailability of ports in the experimental device, the axial position of the LIF and the CW-CRDS measurement locations were not the same. As shown in Figure 2.9, the LIF measurement location is given as the port labeled A while the ports labeled BB' were used for the CW-CRDS measurements. The LIF measurement location is about $5 \mathrm{~cm}$ towards the source with respect to the CW-CRDS measurement location. The CW-CRDS location thus falls outside the strong magnetic field maintained near the source. At this location the magnetic field strength is half of the value at the LIF measurement location. The lack of magnetic field confinement leads to expansion of the plasma, which can also contribute to cooling the plasma and is another possible source of the relatively low ion temperatures as measured by the CW-CRDS 


\section{Chapter 6 References}

${ }^{1}$ J. M. Herbelin, J. A. McKay, M. A. Kwok, R. H. Uenten, D. S. Urevig, D. S. Spencer and D. J. Bernard, Appl. Optics 19, 144 (1980)

2 J. M. Herbelin and J. A. McKay, Appl. Optics 20, 3341 (1981)

${ }^{3}$ P. Elleaume, M. Velghe, M. Billardon, and J. M. Ortega, Appl. Optics 24, 2762 (1985)

${ }^{4}$ D. Z. Anderson, J. C. Frisch and C. S. Masser, Appl. Optics 23, 1238 (1984)

${ }^{5}$ G. Rempe, R. J. Thompson, H. J. Kimble and R. Lalerazi, Optics Lett. 17, 363 (1992)

${ }^{6}$ A. O’Keefe and D. A. G. Deacon, Rev. Sci. Instrum. 59, 2544 (1988)

${ }^{7}$ A. O’Keefe, J. J. Scherer, A. L. Cooksy, R. Sheeks, J. Health and R. J. Saykally, Chem. Phys. Lett. 172, 214 (1990)

${ }^{8}$ J. J. Scherer, J. B. Paul, C. P. Collier and R. J. Saykally, Chem. Rev. 97, 25 (1997)

${ }^{9}$ J. B. Paul, C. P. Collier, R. J. Saykally, J. J. Scherer and A. O’Keefe, Phys. Chem. A. 101, 5211 (1997)

${ }^{10}$ D. Romanini and K. K. Lehmann, J. Chem. Phys. 99, 6287 (1993)

${ }^{11}$ G. Meijer, M. G. H. Boogarts and A.M. Wodtke, Chem. Phys. Lett. 217, 112 (1994)

${ }^{12}$ M. G. H. Boogarts and G. Meijer, J. Chem. Phys. 102, 5269 (1995)

${ }^{13}$ R. T. Jongma, M. G. H. Boogarts, I. Holleman and G. Meijer, Rev. Sci. Instrum. 66, 2821 (1995)

${ }^{14}$ J. Martin et.al., Chem. Phys. Lett. 258, 63 (1996)

${ }^{15}$ J. T. Hodges, J. P. Looney and R. D. van Zee, Appl. Optics 35, 4112 (1996)

${ }^{16}$ J. T. Hodges, J. P. Looney and R. D. van Zee, J. Chem. Phys. 105, 2708 (1996)

${ }^{17}$ L. Lehr and P. Hering, IEEE. J. Quantum Elec. 33, 1465 (1997)

${ }^{18}$ M. Kotterer and J. P. Maier, Chem. Phys. Lett. 266, 342 (1997) 
${ }^{19}$ D. Romanini, J. Gambogi and K. K. Lehmann, in $50^{\text {th }}$ International Symposium on Molecular Spectroscopy (ed. T. A. Miller, Am. Chem. Soc., Ohio State Univ., Columbus, Ohio) (1995)

${ }^{20}$ D. Romanini, A. A. Kachanov, N. Sadeghi and R. Stoeckel, Chem. Phys. Lett. 264, 316 (1997)

${ }^{21}$ K. W. Busch and M. A. Busch, ACS Symposium Series 720, Oxford University Press, DC (1999)

${ }^{22}$ G. Berden, R. Peeters and G. Meijer, Int. Rev. Phys. Chem. 19, 565 (2000)

${ }^{23}$ B. Paldus and A. Kachanov, Can. J. Phys. 83, 975 (2005)

${ }^{24}$ M. Mazurenka, A. J. Orr-Ewing, R. Peverall and G. A. D. Ritchie, Chem. Phys. Lett. 101, 100 (2005)

${ }^{25}$ C. Wang, J. Anal. Atom. Spectrom. 22, 1347 (2007)

${ }^{26}$ G. Friedrichs, Z. Phys. Chem. 222, 31 (2008)

${ }^{27}$ G. Berden and R. Engeln, Cavity Ring-Down Spectroscopy: techniques and application, Wiley, UK (2009)

${ }^{28}$ J. B. Dudek, P. B. Tarsa, A. Velasquez, M. Wladyslawski, P. Rabinowitz and K. K. Lehmann, Anal. Chem. 75, 4599 (2003)

${ }^{29}$ Y.T. Yardley and C. B. Moore, J. Chem. Phys. 45, 1066 (1966)

${ }^{30}$ R. A. Stern and J. A. Johnson, Phys. Rev. Lett. 34, 1548 (1975)

${ }^{31}$ R. J. Pastel and R. C. Sausa, Appl. Opts. 35, 4046 (1996)

${ }^{32}$ W. Gurlit, J. P. Burrows, H. Burkhard, R. Boehm, V. M. Baev and P. E. Toschek, Infrared. Phys. Technol. 37, 95 (1996)

${ }^{33}$ H. Kogelnik and T. Li, Proc. IEEE 54, 1312 (1966)

${ }^{34}$ A. G. Fox and T. Li, Bell Syst. Technol. J. 40, 453 (1961)

${ }^{35}$ K. J. Schulz and W. R. Simpson, Chem. Phys. Lett. 297, 523 (1998)

${ }^{36}$ J. W. Hahn, Y. S. Yoo, Y. J Lee, J. W. Kim and H. W. Lee, Appl. Optics 38, 1859 (1999)

${ }^{37}$ A. M. Keesee, R. Boivin and E. E. Scime, Rev. Sci. Instrum. 75, 4091 (2004)

${ }^{38}$ R. F. Boivin, Zeeman Splitting for LIF Transitions and De-convolution Technique to Extract Ion Temperatures, PL-050, West Virginia University, Morgantown (2002)

${ }^{39}$ E. E. Scime, R. Hardin, A. M. Keesee, C. Biloiu and X. Sun, Phys. Plasmas 14, 043505 (2007) 


\section{CHAPTER 7: Summary}

Measurements of the argon ion velocity distribution function (IVDF) in argon helicon plasma using two different diagnostic techniques (LIF and RFEA) provided important insights into the physics of stable ion beam production by electrostatic double layers in expanding helicon plasmas. ${ }^{1,2}$ By changing only the operating frequency of the source, it was possible to induce a transition from unstable to stable double layer formation. ${ }^{3}$ All other operating parameters, e.g., the magnetic field strength in the source and expansion chamber, the rf power, and the neutral gas pressure (the gas flow rate and the rate of pumping in both HELIX and LEIA) were kept constant. Analysis of the RFEA measurements and the LIF measurements yielded ion beam energies and relative ion beam to background ion densities consistent with each other. The ion beam (and hence the double layer that causes it) formed only when there was relatively weak rf coupling of the helicon antenna to the plasma. When the operating frequency of the source was reduced, there was a modest increase in rf coupling to the plasma that yielded a slightly higher density plasmas and stronger double layers (verified by both the RFEA and LIF measurements). However, below a threshold driving frequency the double layer became unstable and the ion beam disappeared. The upstream plasma density also decreased precipitously. The instabilities that prevented the formation of the stable double layer were investigated with a two-tipped electrostatic probe and further analysis suggests that the fluctuations result from a current driven ion acoustic instability. For cases with a stable ion beam, the instabilities are absent.

Since during the transition between the stable and unstable double layer regimes, the magnetic field or the gas flow or the input power from the amplifier were unchanged, the distinct (and unexpected for regular helicon source operation) increase in plasma density at higher operating source frequencies (where the rf power coupled to the plasma is less and so should have produced less plasma density) could be clearly attributed to the formation of the stable double layer. ${ }^{4}$ Theoretical studies of electrostatic double layers in expanding geometries had predicted ${ }^{5}$ a sharp increase in upstream plasma density with the formation of a double layer. This sudden jump in plasma density is thought to be due to increased upstream ionization by electrons that are accelerated towards the source by 
the double layer (at the same time ions are accelerated out of the source towards the expansion chamber).

We concluded that the ion beam increased in velocity as the operating frequency was decreased until the beam became so strong that it excited the ion-acoustic instability. When the instabilities grew too large, the stable double layer potential structure was not sustainable. Though not described in this work, a series of pulsed source LIF experiments were undertaken to examine the temporal behavior of the phenomena and to understand the causality of the process. ${ }^{6}$ We found that strong beams are initially produced even at lower driving frequencies, but as instabilities grow, the ion acceleration process dies down (indicating the collapse of the double layer). Weaker ion beams were observed to persist throughout the pulsed plasma discharge. Additional spatio-temporal studies of ion beam formation in expanding helicon sources are being carried on using time resolved LIF. $^{7}$

Having used two different diagnostic techniques to measure the IVDF in plasma, we found that there are intrinsic limitations in both of them. The RFEA is very perturbative since it is large, gets very hot in high density plasma, and can only be used in the low density expansion chamber. It is not suitable to use it for source studies in the plasma source itself. Moreover, since the WVU helicon source group has an active research effort in the study of multiple ion species accelerated through double layer, ${ }^{8}$ RFEA (which cannot distinguish between ions of different species) is not an ideal diagnostic. Another disadvantage of an RFEA is that it cannot measure the VDFs of neutrals. In these respects, LIF is a better choice for VDF measurements since it can be used to measure VDFs of either ions or neutral species. Moreover LIF is non-invasive. On the other hand LIF is a very restrictive technique. It is an indirect spectroscopic technique; where information about a particular absorption state is obtained through the fluorescence emission of a third energy state. LIF also requires convenient three or four state schemes along with an available laser with the correct wavelength for the pump transition. For LIF, the initial state should also have a large population for sufficient signal-to-noise; hence initial states are typically ground or metastable states. Thus, for many ions, neutrals, and molecules, LIF is infeasible. A good example of scientifically interesting ion for many plasma sources is helium. Helium ion LIF has been attempted a 
number of times, but the lack of metastable ion state with accessible (by laser) transitions has prevented successful implementation of a LIF scheme. Since collisional quenching can also strongly reduce LIF signal, LIF is effective only when the three or four way schemes $^{9}$ are available in relatively low collisionality plasmas. Finally, since LIF is typically an excited state measurement, complex models and additional measurements of plasma parameters are generally required to determine the bulk ion or neutral density from the LIF signal intensity.

As alternative to LIF, we have demonstrated that continuous wave cavity ring down spectroscopy (CW-CRDS) ${ }^{10}$ provides a new means of measuring IVDFs in plasmas. CW-CRDS is a proven ultra-sensitive cavity enhanced absorption technique and combined with cheap and easily available diode lasers that have sufficient resolution to resolve Doppler broadened line shapes; it can be used to measure a VDF in plasma. Being a spectroscopic method, it is non-invasive and does not require a three or four state scheme, a single absorption transition is all that is required. The signal in a CW-CRDS experiment is an absolute measure of the density of the target species. These features make CW-CRDS attractive as an alternate technique for IVDF measurements. Though CW-CRDS was discovered in the late 1980s, the complexities of achieving CW-CRDS in very low pressure systems prevented its use by the plasma physics community (other than in atmospheric plasmas, which do not need the optical cavity to be mounted on an evacuated chamber). ${ }^{11}$ Even though pulsed CRDS $^{12}$ is more common, it suffers from a lack of sensitivity due to multimode excitation of the cavity. In this work we have demonstrated that CW-CRDS can be, in fact, used to measure a Doppler broadened VDF in plasmas. In addition to the information from typical LIF measurements of the IVDF, we could also calculate the absolute metastable state density of the ions.

These preliminary CW-CRDS measurements highlighted a number of failings of the current implementation of the technique. The most significant problem was the degradation of the mirrors with time. The use of a thin layer of an inert buffer gas in front of the mirrors has been suggested as a method to prevent mirror damage. ${ }^{13}$ The present experimental configuration also suffers from noise due to vibrations from the pumps in the system. A separate housing for the pumps would help to eliminate this problem. Finally, these initial experiments were accomplished using physically separate injection 
optics, optical cavity optics, and collection optics. A single optical chain could eliminate many of the challenges in aligning the apparatus.

Even though improvements are required to make CW-CRDS a routine plasma diagnostic, the technique does work. Armed with this new approach to VDF measurements it should be possible to measure IVDFs of species that heretofore have been inaccessible by LIF techniques and in conjunction with LIF it may be possible to obtain both absolute density and temperature measurements of a target species. 


\section{Chapter References}

${ }^{1}$ X. Sun, A. M. Keese, C. Biloiu, E. E. Scime, A. Meige, C. Charles and R. W. Boswell, Phys. Rev. Lett. 95, 025004, (2005)

${ }^{2}$ Z. Harvey, S. Chakraborty Thakur, A. Hansen, R. A. Hardin, W. S. Przybysz and E. E. Scime, Rev. Sci. Instrum. 79, 10F314 (2008)

${ }^{3}$ S. Chakraborty Thakur, A. Hansen and E. E. Scime, Plasma Sources Sci. Technol. 19, 025008 (2010)

${ }^{4}$ S. Chakraborty Thakur, Z. Harvey, I.A. Biloiu, A. Hansen, R. A. Hardin, W. S. Przybysz and E. E. Scime, Phys. Rev. Lett. 102, 035004 (2009)

${ }^{5}$ M. A. Lieberman and C. Charles, Phys. Rev. Lett. 97045003 (2006)

${ }^{6}$ E. E. Scime, I. A. Biloiu, J. Carr Jr., S. Chakraborty Thakur, M. Galante, A. Hansen, S. Housmandyar, A. M. Keesee, D. McCarren, S. Sears, C. Biloiu and X. Sun, Phys. Plasmas 17, 055701 (2010)

${ }^{7}$ J. Carr Jr. et. al., (in preparation)

${ }^{8}$ I. A. Biloiu, Laser Induced Fluorescence Studies of Ion Acceleration in Single and Multiple Species Expanding Plasmas, Ph. D. Thesis, West Virginia University, Morgantown (2009)

${ }^{9}$ A. M. Keesee, R. Boivin and E. E. Scime, Rev. Sci. Instrum. 75, 4091 (2004)

${ }^{10}$ D. Romanini, A. A. Kachanov, N. Sadeghi and R. Stoeckel, Chem. Phys. Lett. 264, 316 (1997)

${ }^{11}$ C. Wang, J. Anal. Atom. Spectrom. 22, 1347 (2007)

${ }^{12}$ A. O’Keefe and D. A. G. Deacon, Rev. Sci. Instrum. 59, 2544 (1988)

${ }^{13}$ G. Berden, R. Peeters and G. Meijer, Int. Rev. Phys. Chem. 19, 565 (2000) 


\section{APPENDIX 1: Details of the threshold detector tracking circuit:}

The electronic "threshold detector" tracking circuit, briefly described in Section 6.3.2 (page 169) of the thesis, is an essential part of the CW-CRDS set up. The circuit has four different circuit components: voltage comparator, pulse generator, adder and inverter, as shown schematically in Fig. 6.15 (page 170) of the thesis.

The first component is the voltage comparator. This is achieved using LM 339N, which is a quad single supply comparator chip. A simple voltage threshold detector circuit is shown below. The comparator can be either inverting (CIRCUIT - A) or noninverting (CIRCUIT - B). We use the inverting comparator circuit, such that whenever the input voltage becomes greater than the set reference voltage (here, $V_{\text {ref }}=V / 2$ ), the output goes negative.

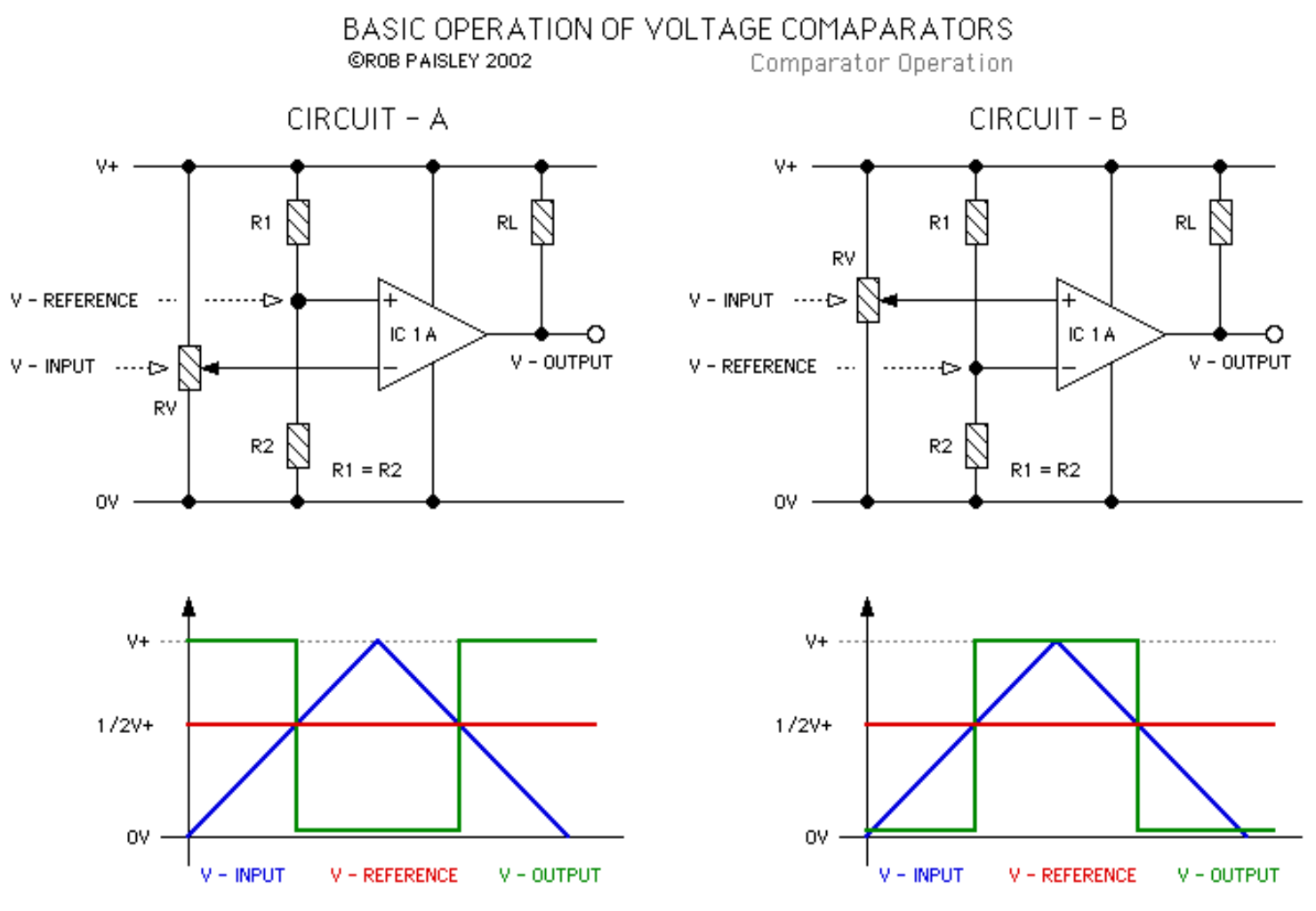

http://home.cogeco.ca/ rpaisley4/CircuitIndex.html

The following link contains the data sheet of LM339N giving all the details of the integrated chip including the pin diagram and the voltage specifications:

(http://pdf1.alldatasheet.com/datasheet-pdf/view/3071/MOTOROLA/LM339N.html) 
The second circuit component is a pulse generator built by using a NE555P timer. The 555 timer is triggered by the negative going pulse of the inverting voltage comparator, whenever the input voltage from the photo-detector becomes greater than the $\mathrm{V}_{\text {ref. }}$ The circuit is designed such that the 555 timer works in the mono-stable operation, which mean that on triggering, the 555 timer shall generate only one pulse and then the output voltage goes to zero. The circuit diagram shown below represents the action of the 555 timer, as intended in our experiment. The negative going input pulse produces the positive going output pulse, where the width of this pulse can be externally controlled by using a potentiometer $(\mathrm{R})$. The width of the output pulse is given approximately by $\mathrm{T}=$ 1.1RC. Accordingly the resistor and the capacitors were chosen. As shown in the characteristics below, once the 555 is triggered, further trigger pulses are completely ignored during the lifetime of the output pulse.
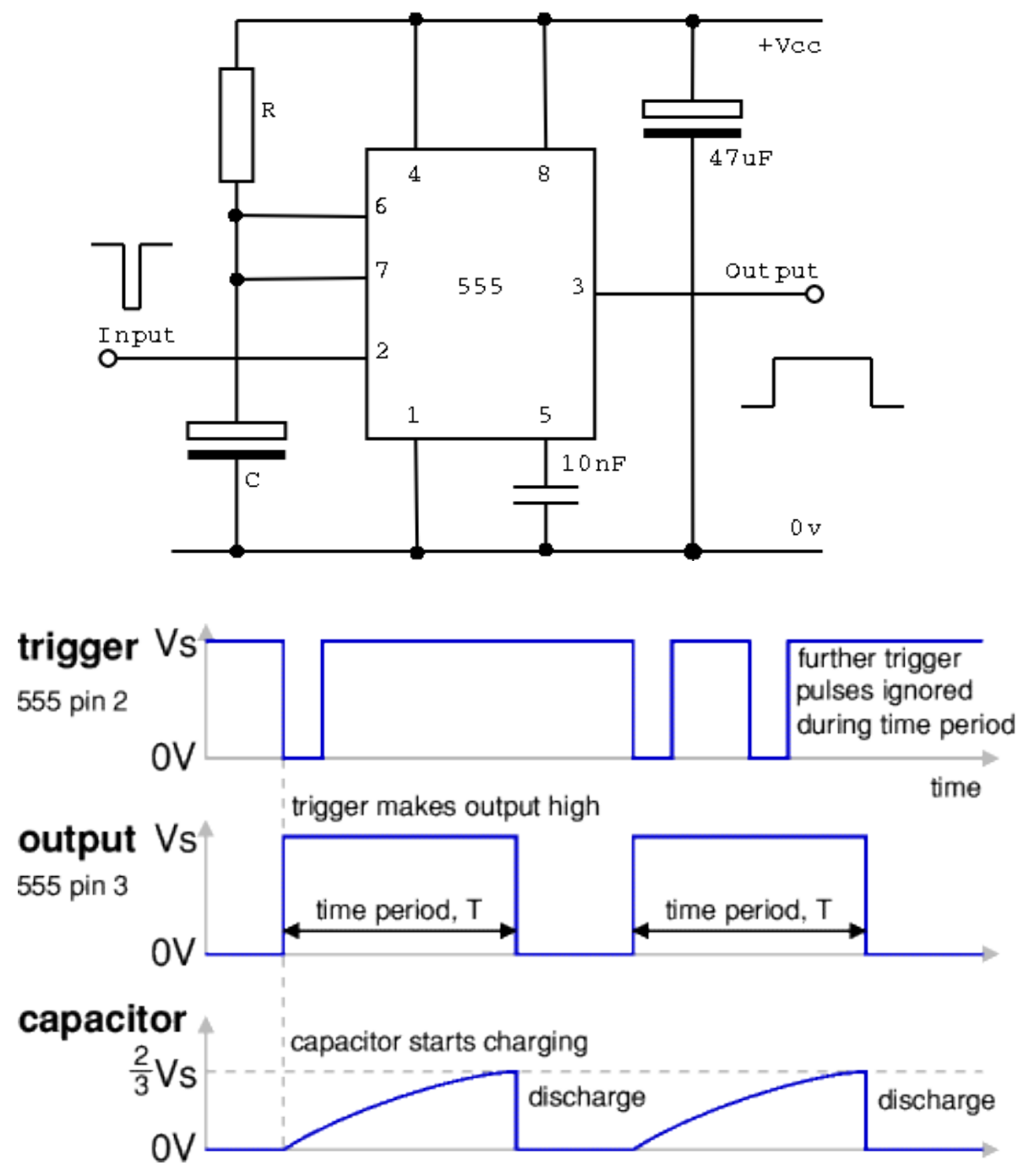
The following link contains the data sheet of NE555P giving all the details of the integrated chip including the pin diagram and the voltage specifications: (http://www.datasheetcatalog.com/datasheets_pdf/N/E/5/5/NE555P.shtml)

The third circuit component is an adder which effectively "adds" the output signal from the 555 pulse generator to the TTL square wave pulses that are used to drive the AOM. The addition is in time space and not in voltage. To achieve this, we use the 74HC32N chip, which is a quad two input OR gate. The OR gate is a digital logic gate that has two inputs and one output. The output is high if one or both of the inputs are high.

The following link contains the data sheet of 74HC32N giving all the details of the integrated chip including the pin diagram and the voltage specifications: (http://www.alldatasheet.com/datasheet-pdf/pdf/98370/PHILIPS/74HC32N.html)।

The fourth circuit component is a NOT gate, which effectively inverts the input signal and then the inverted output is sent to the AOM. To achieve this, we use the 74HC04N chip, which is a hex inverter. The NOT gate is a digital logic gate that has one input and one output. The output is simply the inverted version of the input.

The following link contains the data sheet of 74HC04N giving all the details of the integrated chip including the pin diagram and the voltage specifications: (http://www.alldatasheet.com/datasheet-pdf/pdf/106276/PHILIPS/74HC04N.html)

The actual circuit used is thus a simple series [in a single chain] combination of all the four circuit components described above. The expected working of the circuit is shown in Figure 6.16 (page 172) of the thesis, by generating a model voltage signal pulse from the signal generator and measuring the voltage output signals at each individual circuit component. The parameters [rise time and pulse width] of the model signal pulse generated was chosen based on previous measurements of the actual mirror cavity transmission characteristics. We found that for the optical cavity used, based on the mirror reflectivity and the cavity length, the rise time of the optical resonances were about $1 \mathrm{msec}$ and were sustained for about 20 msecs. So accordingly, we chose the model 
signal pulse with a rise time of 100 nsecs (the maximum that was allowed in the function generator used) and pulse-width of 20 msecs. We found that the tracking circuit took a couple of milliseconds to send the final signal to the AOM to turn it off (with respect to when the generated signal is initiated). But the AOM shut off in a few hundred nanoseconds, much faster than our expected ring down time scales.

NOTE: The last two circuit components can be effectively replaced by a single component called a NOR gate. The NOR gate acts as an OR gate followed by a NOT gate in the same integrated chip. One such chip is the 74HC02N, which is a quad two input NOR gate.

(http://www.alldatasheet.com/datasheet-pdf/pdf/15525/PHILIPS/74HC02N.html) 
Saikat Chakraborty Thakur

\section{Education}

Ph.D. Plasma Physics December 2010

West Virginia University, Morgantown, WV 26506

M.Sc. Physics May 2004

Indian Institute of Technology [IIT], Kanpur, India

B.Sc. Physics July 2002

Calcutta University [St. Xavier's College], Kolkata, India

\section{Research Interests}

- Plasma spectroscopy [passive, cavity enhanced, laser aided]

- Magnetically confined fusion plasma

- Laser based plasma

- Plasma diagnostics

- Helicon plasma

- Atmospheric plasma discharges

- Nonlinear dynamics

- Plasma simulation

\section{Research Experience}

Spring 2008 - Summer 2010

- Continuous Wave Cavity Ring Down Spectroscopy [cw-CRDS]; an ultrasensitive cavity enhanced absorption technique

- Studies of Double Layers [DL] in expanding argon helicon plasmas

Fall 2007

- Spatio-temporal periodic pulling in a neon glow discharge plasma

- Numerical modeling of the Van der Pol behavior of relaxation oscillations in a periodically pulled thermionic discharge

Summer2006 - Summer 2007

- Setting up the Blaamann Simple Magnetized Torus [SMT] in WVU

Summer 2004

- High power timer circuit development for confinement studies by controlled edge biasing of the SINP [Saha Institute of Nuclear Physics] Tokamak 
Fall 2003 - Spring 2004

- Environmental and light induced effects on 8-Hydroxy Quinoline derivative metal complex small molecules

- Electrical transport and optical properties of organic semiconductor thin films

- Organic luminescent small molecule LEDs: green and blue emitters

- Fabrication of organic solar cells

Summer 2003

- Detection of plasma impurities [heavy ions like tungsten, molybdenum, Fe-I and Fe-II in addition to $\mathrm{H}, \mathrm{O}$ and $\mathrm{C}$ ions in various states of ionization] in the SINP Tokamak using optical emission spectroscopy

- Detected O-V lines in the SINP Tokamak for the first time ever

- Non-invasive measurement of plasma temperature using hydrogen and oxygen lines in the SINP Tokamak

Spring 2003

- Experimental verification of the "Bouncing ball problem and the bifurcation route to chaos"

- Experimentally observing chaos in a double pendulum

Fall 2002

- Numerical simulation studies of a non-linearly damped, driven harmonic oscillator [Mathieu's Equation]

\section{Teaching Experience}

Fall 2007 - Spring 2008 and Fall 2008 - Spring 2009

- Head Graduate Teaching Assistant

Fall 2004 - Spring 2006

- Graduate Teaching Assistant

\section{Graduate Courses within Research Concentration}

- Principles of Plasma Physics

- Advanced Kinetic Theory of Plasma

- Computational Plasma Physics

- Advanced Magnetohydrodynamic [MHD] Theory of Plasma

- Nonlinear Dynamics 


\section{Additional Professional Experience}

- CMPD/CMSO Winter School, Plasma Turbulence and Transport, UCLA 2007

- Editor and M.Sc. representative, Eigen Space - Physics Wall Magazine, IIT Kanpur, 2003-04

- Editor, Prayas - Student's Journal of Physics, a bi-monthly journal published by Indian Association of Physics Teachers [IAPT], 2003-04

- Indo-Italian Workshop on Organic Semiconductors [IIWOS], IIT Kanpur, 2003

- Successfully completed the Physics Summer School, Indian Association for the Cultivation of Science [IACS], Kolkata, 2000

\section{Awards and Honors}

- Chosen as the "Head Graduate Teaching Assistant", WVU 2008-09

- Carl Rotter award for the "Best Graduate Teaching Assistant”, WVU, 2008-09

- Chosen as the "Head Graduate Teaching Assistant", WVU, 2007-08

- Carl Rotter award for the "Best Graduate Teaching Assistant", WVU, 2005-06

- "Most Outstanding Graduate Teaching Assistant" award, WVU, 2004-05

- Best M.Sc. Project award, IIT Kanpur, 2003-04

- $3^{\text {rd }}$ Best Poster, $5^{\text {th }}$ Condensed Matter Workshop, IIT Kanpur, 2004

- Merit-Cum-Means [MCM] Scholarship, IIT Kanpur, 2003-04

- Best all round performance award, St. Xavier's College Hostel, 2000-01

- Best all round performance award, St. Xavier's College Hostel, 1998-99

\section{Talks and Colloquia}

- Improved confinement of pure electron plasma in the Lawrence Non-Neutral Torus [LNT-II], West Virginia University, 2009

- Discovery of the flickering auroral roar, West Virginia University, 2007

- Fusion and tokamak plasmas, St. Xavier’s College, Kolkata, 2004

- Electrical transport properties of organic small molecules, IIT Kanpur, 2004

- Spectroscopy of plasma impurities, IIT Kanpur, 2004

- Bouncing ball problem and the route to chaos, SINP, Kolkata, 2003

- Electrostatic analog of stretched membranes, St. Xavier’s College, Kolkata, 2002

- Measuring the surface tension of water, St. Xavier's College Kolkata, 2001 


\section{Publications}

\section{$\underline{\text { Refereed Journals }}$}

- S. Houshmandyar, S. Chakraborty Thakur, S. Sears, J. Carr Jr., E. E. Scime "Observation of neutral depletion in high power helium helicon plasmas", (submitted to Reviews of Scientific Instruments)

- J. Carr Jr., S. Chakraborty Thakur, A. Hansen, S. Houshmandyar, S. Sears and E. E. Scime, "Time-resolved studies of ion beam formation in pulsed helicon argon plasma”, (in preparation)

- S. Chakraborty Thakur and W. Abdul-Razzaq, "Voltage generated across the wings of an airplane”, (submitted to Am. Journal of Physics)

- S. Chakraborty Thakur, A. Hansen, R. A. Hardin and E. E. Scime, "Threshold for formation of a stable double layer in expanding helicon plasma," (submitted to Plasma Sources Sci. Technol.)

- E. E. Scime, I. A. Biloiu, J. Carr Jr., S. Chakraborty Thakur, M. Galante, A. Hansen, Z. Harvey, S. Houshmandyar, A. M. Keesee, D. McCarren, W. S. Przybysz, S. Sears, C. Biloiu and X. Sun, "Time-resolved measurements of double layer evolution in expanding plasma,” Phys. Plasmas 17, 055701 (2010)

- W. S. Przybysz, J. Ellis, S. Chakraborty Thakur, A. Hansen, R. A. Hardin, S. Sears and E. E. Scime, "A Magneto-Optic probe for magnetic fluctuation measurements,” Rev. Sci. Instrum. 80103502 (2009)

- S. Chakraborty Thakur, Z. Harvey, I. A. Biloiu, A. Hansen, R. A. Hardin, W. S. Przybysz and E. E. Scime, "Increased upstream ionization due to formation of a double layer,” Phys. Rev. Lett. 102, 035004 (2009)

- Z. Harvey, S. Chakraborty Thakur, A. Hansen, R. Hardin, W. S. Przybysz and E. E. Scime, "Comparison of gridded energy analyzer and laser induced fluorescence measurements of a two-component ion distribution," Rev. Sci. Instrum. 79, 10F314 (2008)

- G. M. Paily, S. Chakraborty Thakur, and S. R. P. Mohapatra, "Bouncing ball problem and the route to chaos,” Prayas - Student's Journal of Physics: IAPT 1, 28 (2004) 


\section{Conference Presentations}

- S. Chakraborty Thakur, J. Carr Jr. and E. E. Scime "Cavity ring down spectroscopy measurements of Ar-II velocity distribution functions”, APS - Div. of Plasma Physics (2009)

- J. Carr Jr., S. Chakraborty Thakur, A. Hansen, S. Houshmandyar, S. Sears and E. E. Scime, "Time resolved studies of ion beam formation in pulsed helicon Argon plasma”, APS - Div. of Plasma Physics (2009)

- S. Houshmandyar, S. Chakraborty Thakur, S. Sears, J. Carr Jr., E. E. Scime "Observation of neutral depletion in high power Helium helicon plasmas", APS Div. of Plasma Physics (2009)

- S. Sears, S. Houshmandyar, J. Carr Jr., M. Galante, K. Tallaksen, S. Chakraborty Thakur and E. E. Scime, "Electric field measurements in a helicon source based on Stark splitting of neutral Helium lines”, APS - Div. of Plasma Physics (2009)

- S. Chakraborty Thakur, Z. Harvey, I. A. Biloiu, A. Hansen, R. A. Hardin, W. S. Przybysz and E. E. Scime, "Observation of increased upstream ionization resulting from spontaneous formation of a double layer in an expanding Argon helicon plasma”, ICOPS (2009)

- S. Chakraborty Thakur, Z. Harvey, I. A. Biloiu, A. Hansen, R. A. Hardin, W. S. Przybysz and E. E. Scime, "Frequency threshold for ion beam formation in expanding RF plasma”, APS - Div. of Plasma Physics (2008)

- J. Ellis, J. Carr Jr., S Chakraborty Thakur, R. A. Hardin, W. S. Przybysz and E. E. Scime, "Intensity threshold circuit for cavity ring down spectroscopy diagnostic”, APS - Div. of Plasma Physics (2008)

- Z. Harvey, S. Chakraborty Thakur, A. Hansen, R. Hardin, W. Przybysz and E. Scime, "Comparison of gridded energy analyzer and laser induced fluorescence measurements of a two-component ion distribution”, HTPD (2008)

- V. K. Shukla, D. Dutta, S. Chakraborty Thakur, S. Saxena and S. Kumar, "Ellipsometry of display materials”, Samtel Center for Display Technol. (2004)

- D. Dutta and S. Chakraborty Thakur, "Electrical and optical properties of organic semiconductor thin films”, $5^{\text {th }}$ Cond. Matt. Workshop, Kanpur (2004)

- V. K. Shukla, D. Dutta, S. Chakraborty Thakur, S. Saxena and S. Kumar, "Organic luminescent small molecules: green and blue emitters”, Samtel Center for Display Technol. (2004) 
- V. K. Shukla, D. Dutta, S. Chakraborty Thakur and S. Kumar "Environmental and light induced effects on 8-Hydroxy Quinoline derivative metal complex small molecules”, Indo-Italian Workshop on Organic Semiconductors (2003)

\section{Professional Membership}

- American Physical Society [APS]

- Division of Plasma Physics [DPP]

- Institute of Electrical and Electronics Engineers [IEEE]

- Nuclear and Plasma Sciences Society [NPSS]

- American Association of Physics Teachers [AAPT]

- Sigma Pi Sigma - National Physics Honor Society

- Indian Association of Physics Teachers [IAPT]

\section{References}

- Prof. Earl E. Scime, Department of Physics, West Virginia University, Morgantown, WV 26505-6315 (escime@wvu.edu)

- Prof. Arthur H. Weldon, Department of Physics, West Virginia University, Morgantown, WV 26505-6315 (hweldon@wvu.edu)

- Prof. Boyd F. Edwards, Department of Physics, West Virginia University, Morgantown, WV 26505-6315 (bedwards@wvu.edu) 